\title{
Towards Optical Cochlear Implants: Behavioral and Physiological Responses to Optogenetic Activation of the Auditory Nerve
}

\author{
Dissertation \\ for the award of the degree \\ "Doctor rerum naturalium" \\ of the Georg-August-Universität Göttingen \\ within the IMPRS for Neurosciences \\ of the Georg-August University School of Science (GAUSS) \\ submitted by \\ Alexander Dieter \\ from Seligenstadt
}





\section{Thesis Committee:}

Prof. Dr. Tobias Moser

Institute for Auditory Neuroscience, University Medical Center Göttingen

Prof. Dr. Tim Gollisch

Department of Ophthalmology, University Medical Center, Göttingen

Prof. Dr. Stefan Treue

Cognitive Neuroscience Laboratory, German Primate Center, Göttingen

\section{Members of the Examination Board:}

Prof. Dr. Martin Göpfert

Department of Cellular Neurobiology, Schwann Schleiden Research Center, Göttingen

Prof. Dr. Hansjörg Scherberger

Neurobiology Laboratory, German Primate Center, Göttingen

Camin Dean, PhD

Trans-synaptic Signaling, European Neuroscience Institute, Göttingen

Date of oral examination: November, $8^{\text {th }}, 2019$ 



\section{Table of Contents}

Abstract

Introduction

Sensory systems and the importance of audition - a personal note....................................... 3

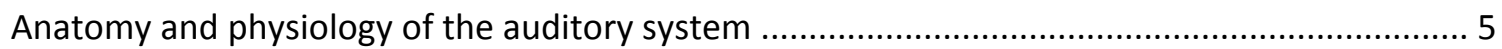

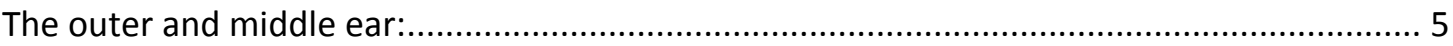

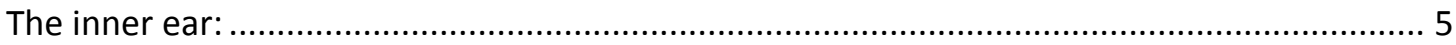

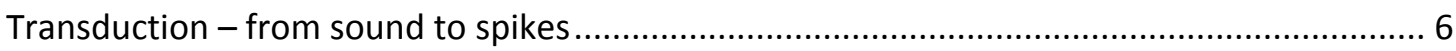

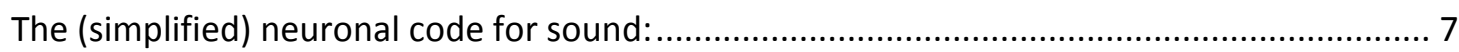

The central auditory pathway and auditory plasticity: ...................................................... 9

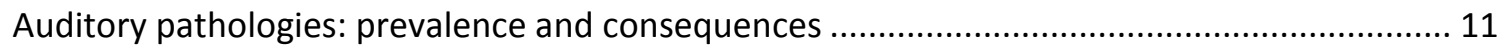

Re-building audition: restoration of sensorineural hearing loss.............................................. 12

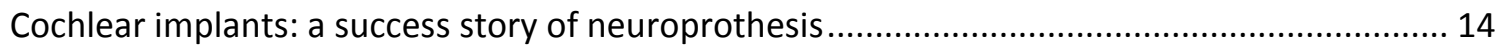

Overcoming the bottlenecks: towards optical cochlear implants .......................................... 16

Scope of this thesis: validating cochlear optogenetics in adult Mongolian gerbils ..................... 20

Perception of cochlear optogenetics and optogenetic hearing restoration:.......................... 20

Comparison of spectral selectivity upon optogenetic and electrical SGN stimulation:........... 21

In vivo validation of $\mu$ LED based optical cochlear implants:.............................................. 21

Chapter I: Optogenetic Stimulation of cochlear neurons activates the auditory pathway and restores auditory driven behavior in deaf adult gerbils...................................................................... 23

Author contributions:

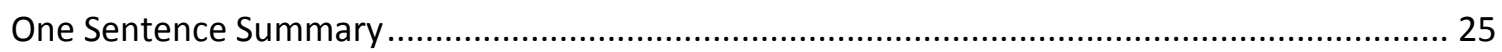

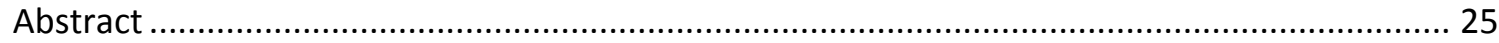

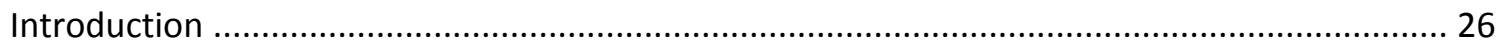

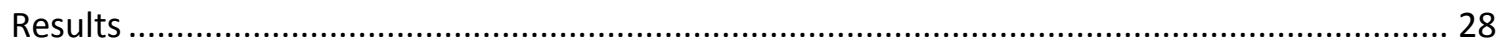




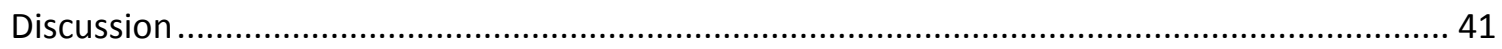

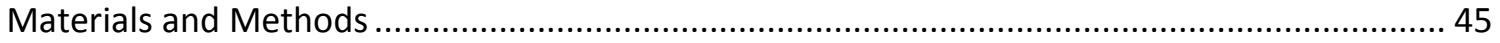

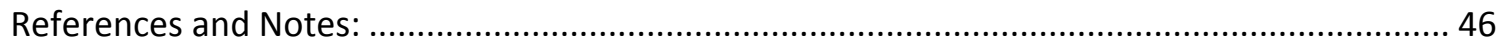

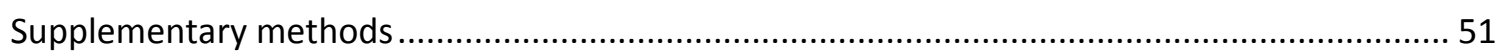

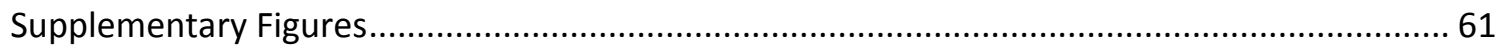

Chapter II: Near physiological spectral selectivity of cochlear optogenetics................................. 73

Author contributions:

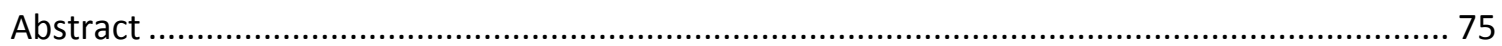

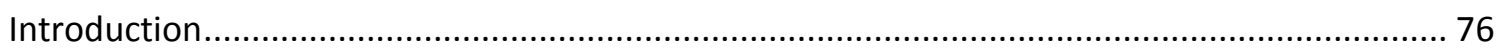

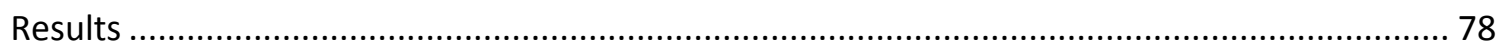

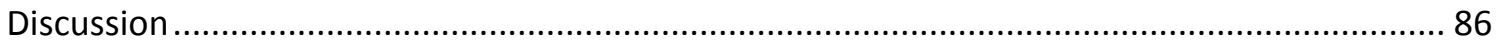

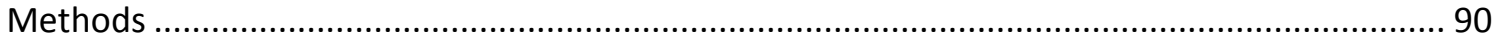

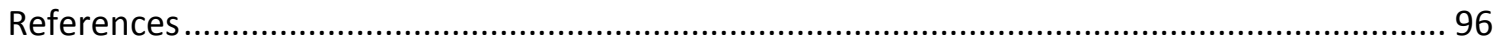

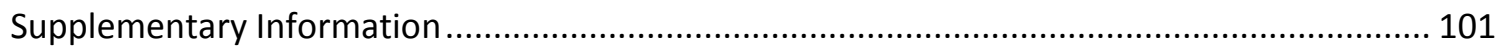

Chapter III: $\mu$ LED-based optical cochlear implants for spectrally selective activation of the auditory nerve

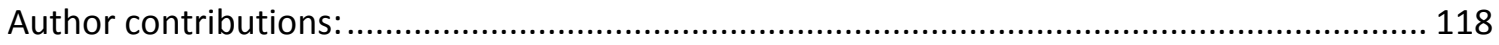

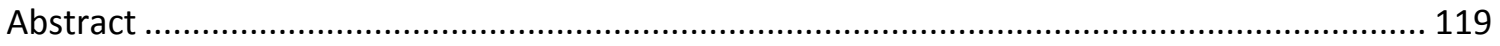

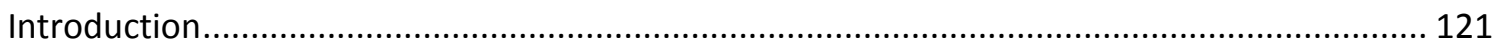

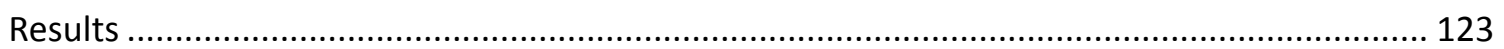

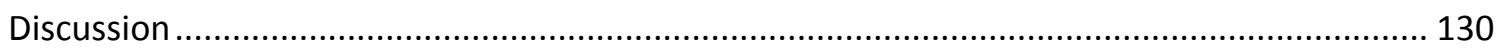

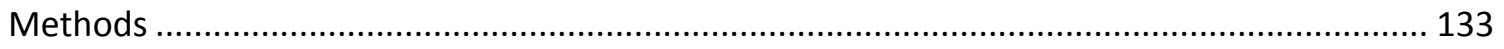

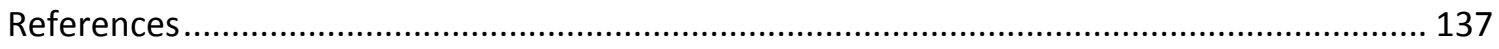

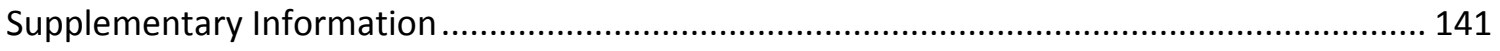




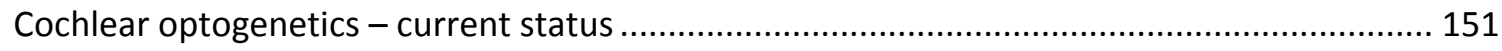

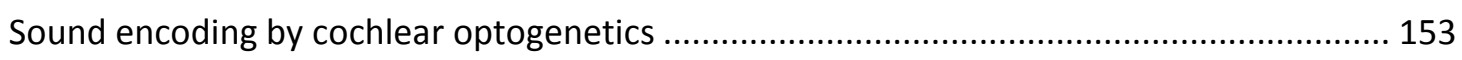

Cochlear optogenetics for hearing restoration .............................................................. 158

Engineering of multi-channel optical cochlear implants.................................................. 161

The roadmap towards clinical translation of optogenetic cochlear implants ............................ 164

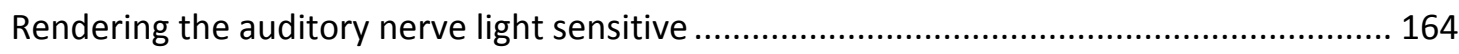

Evaluating the biosafety of cochlear optogenetics ............................................................ 167

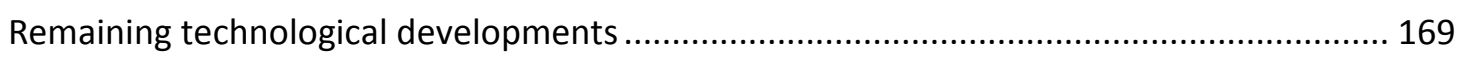

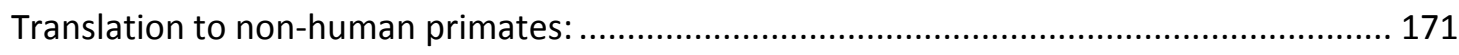

5.3 Cochlear optogenetics as a tool for auditory neuroscience ............................................. 173

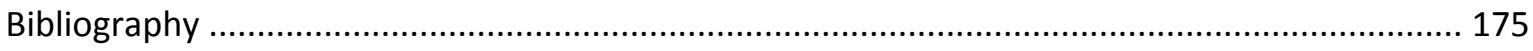

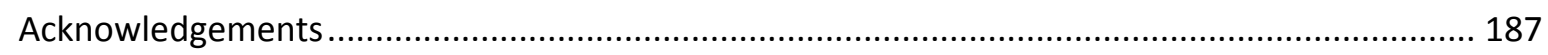

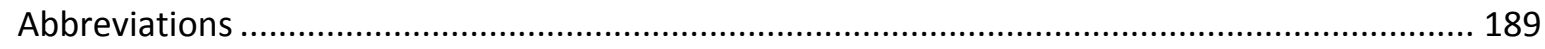

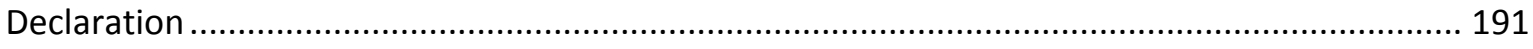







\begin{abstract}
Cochlear implants ( $\mathrm{Cls}$ ) constitute the interface between the sound-deprived brain of patients suffering from sensorineural hearing loss and the auditory scene surrounding them. By electrically stimulating the auditory nerve (AN), Cls mimic coding principles of the cochlea and provide the user with auditory information, enabling speech comprehension in half a million implantees. Unfortunately, current is hard to steer in the cochlear fluids, limiting the spatial selectivity and thus the spectral resolution of electrical hearing restoration. As light can be conveniently confined in space, optogenetic stimulation of the genetically modified AN might overcome this limitation. Indeed, cochlear optogenetics with high temporal fidelity was demonstrated upon viral gene transfer of ultrafast Channelrhodopsins in early postnatal mice, and field potentials in the auditory midbrain of transgenic mice indicated increased spectral selectivity of cochlear optogenetics. Furthermore, optical cochlear implants (oCls) based on light emitting diodes have been developed for multi-site illumination of the AN. However, a long way remains to be gone before considering clinical translation. Working with adult Mongolian gerbils whose AN has been virally transduced with the Channelrhodopsin-variant CatCh, this thesis addresses three milestones towards the development of cochlear optogenetics: First, perception of optogenetic AN activation has been demonstrated on the behavioral level, which is essential when considering that oCls must convey behaviorally relevant information to future users. Second, cochlear optogenetics has been demonstrated to activate the auditory pathway in a tonotopic manner and with increased spectral selectivity as compared to mono- and bipolar electrical stimulation. This finding is of uppermost importance, since clinical translation of cochlear optogenetics is only justified if a substantial advantage of optogenetic over electric sound encoding is to be expected. Third, virus-mediated gene transfer in adult gerbils has been combined with microsystems technology to facilitate multi-channel optogenetic AN activation. 16-channel oCls based on microscale light emitting diodes enabled AN activation with higher spectral selectivity as compared to electrical stimulation, and hence demonstrate the feasibility of a translational oCl approach. Furthermore, functional activation of the AN by optogenetics has been demonstrated in a gerbil model of sensorineural hearing loss, both on the physiological and behavioral level. These results suggest that optogenetic hearing restoration is behaviorally relevant and might indeed overcome the major bottleneck of electrical Cls, raising hope that the quality of artificial sound encoding for future patients might be improved by optical cochlear implants.
\end{abstract}




\section{$\underline{\text { Introduction }}$}

\section{"The brain is the most important organ, according to the brain" - Unknown}

Starting my PhD thesis with this quote allows me to briefly share my enthusiasm for neuroscience with the reader: While I do not necessarily believe that the brain is the most important organ of the body, it is arguably the most complex and fascinating organ of the body. Not only does it allow us to navigate, forage and reproduce successfully in a complex environment, but also attributes such as emotions, high level cognition, and speech are rooted in the brain. Without our brains there would be nothing like relationships, visions or the thrilling joy of reward, to name only a few. These features not only make our lives enjoyable, but are also key features of neuroscientific research (and research in general), which in turn helps us to understand the organ that gives rise to these features. If brain function is disturbed, all of this is at risk - reason enough to motivate tens of thousands of researchers worldwide to understand the brain in health and disease, as well as finding ways to restore normal brain function. Advances in hard- and software technology as well as tremendous funding - due to growing public and economic interest in neuroscience make the $21^{\text {st }}$ century a golden age for neuroscientific research and I am really grateful to be part of this movement and contribute my tiny share in understanding the brain and treating neuronal dysfunction.

\section{Sensory systems and the importance of audition - a personal note}

Breaking the brain down to the very basics, one could argue that the brain is our survival-organ. By integrating various sensory inputs originating from the external world with inherited instincts and acquired experience of the brains internal world, it produces goal-oriented behavior necessary to survive and reproduce. To do so, different organisms rely on a variety of highly specialized senses, which allow them to accomplish this demanding task under their individual circumstances and in their individual environments. In case of primates - including humans - the basic senses which collect environmental inputs are olfaction, gustation, somatosensation, vision and audition (even though we actually have more senses, e.g. balance or proprioception). Even 
though all of these senses are of great importance, I still want to make a case for audition: while olfaction, gustation and somatosensation are senses which scan the external world in close proximity to the observer, vision and audition collect also cues from far away and thus greatly extend the area that the organism interacts with. Both of these senses contribute tremendously to the basic functions that make the organism survive - like the detection of potential nourishment and predators as well as communication and reproduction with conspecifics. While vision - at least for primates - might be more important for a fast scan of the environment and thus improves the chances to survive for each individual, audition enables precise and efficient information exchange between individuals, and thus extends the environment that the organism interacts with also to the environment that conspecifics share their information about (both in space and in time). The communication humans developed during their evolution is a basic requirement for networking and developing sophisticated culture, ultimately leading to the highly complex man-environment that we live in. For this reason I believe that the sense of audition played a crucial role in shaping nowadays world, which makes it a fascinating object to study (beyond - of course - the obvious reasons that interest a sensory neuroscientist). 


\section{Anatomy and physiology of the auditory system}

\section{The outer and middle ear:}

Each detector - which sensory systems essentially are - is specialized to detect a specific signal from the physical world. In case of audition, the adequate signals are pressure waves, i.e. density differences in the surrounding medium which fluctuate in time. These pressure waves are collected by the pinna of the outer ear and directed on the ossicular chain in the middle ear via the ear canal and the tympanic membrane (figure $1 \mathrm{~A}$ ). The ossicles - namely malleus, incus and stapes - convey pressure waves of the surrounding medium (typically air) arriving at the tympanic membrane into pressure waves in the fluid filled cochlea. Due to their anatomy, ossicles amplify pressure waves about 20-fold (via leverage and differences in the dimensions of the tympanic membrane and the oval window), enabling the transition of pressure waves from the surrounding medium into the cochlear fluid.

\section{The inner ear:}

The cochlea itself, a snail-shaped, bony structure, consists of three distinct fluid-filled cavities (scala tympani, delimited via the basilar membrane from the scala media, which in turn is delimited via Reissner's membrane from the scala vestibuli) coiled around the central pillar of the cochlea, the modiolus. At the cochlear apex, the scala tympani and the scala vestibuli are connected via the helicotrema, and both are filled with a liquid called perilymph. At the cochlear base, two membrane-covered windows are found: the oval window in the scala vestibuli and the round window in the scala tympani. When the stapes moves, it also moves the oval window membrane; perilymph in the scala vestibuli is pushed towards the cochlear apex and in turn pushes the perilymph in the scala tympani via the helicotrema towards the round window, which is bulged outward (figure 1B). These pressure waves cause motion while traveling through the cochlea, most importantly at the basilar membrane. Due to its physical properties - it gradually increases in width and decreases in stiffness from the cochlear base towards the apex - its displacement depends on the frequency of pressure waves: While high frequencies deflect the stiff and narrow part at the cochlear base, low frequency waves travel up to the cochlear apex and mainly deflect the wider and more flexible part of the basilar membrane. As a result, a placefrequency code is established in the cochlea, where different frequencies of pressure waves (originating from different frequencies of sound) cause basilar membrane deflections at different cochlear locations - a phenomenon of great importance that will accompany the reader throughout this thesis. 


\section{Transduction - from sound to spikes}

The responsible organ for signal transduction - the organ of Corti - is located on the basilar membrane in the scala media. It is covered by a second membrane, the tectorial membrane, which is connected to the basilar membrane via the rods of Corti (pillar cells). At its top, the organ of Corti faces the potassium-rich endolymph, and at its base the perilymph, which resembles regular extracellular saline. The sensory cells responsible for neural transduction are called hair cells. Hair cells are embedded in a variety of supporting cells and can be separated into approximately 3500 inner (in between the modiolus and the rods of Corti) and 15-20,000 outer hair cells (distal to the rods of Corti; figure 1C). Hair cells are named after the 100 stereocilia which are located at the top of each cell and extend into the tectorial membrane (in the case of outer hair cells) or just below the tectorial membrane (inner hair cells). Stereocilia are anatomically connected via tip-links and house mechanically gated cation channels. In resting state these channels are partially open, resulting in modest potassium influx from the endolymph
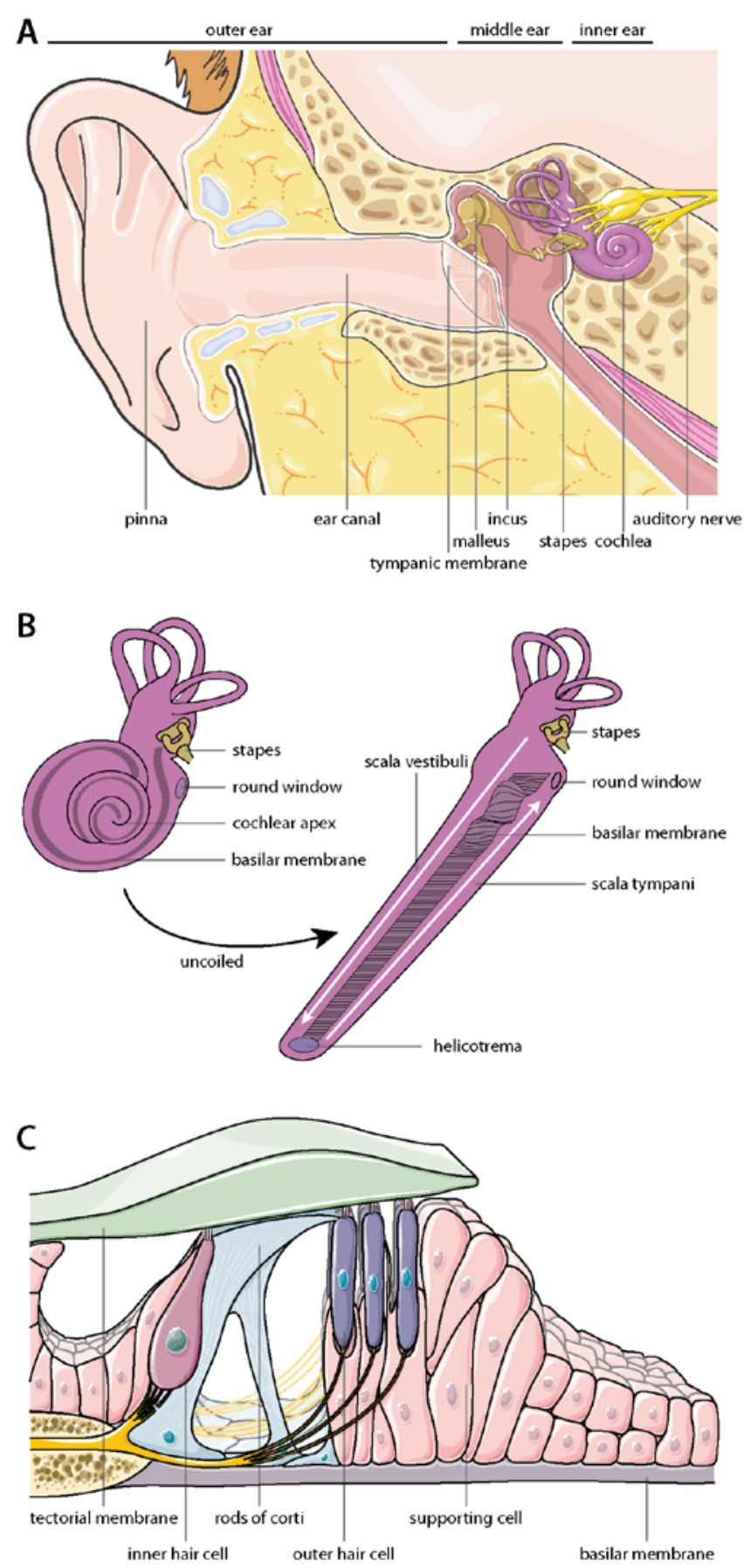

Figure 1: Anatomy of the ear. (A) Illustration of the human ear. (B) Illustration of the inner ear in its natural shape (left) and in a hypothetical uncoiled state (right). White arrows in the uncoiled cochlea indicate the travelling pressure wave, starting at the oval window under the footplate of the stapes. (C) Illustration of the organ of Corti. Panel A and Panel $\mathrm{C}$ have been taken and partially modified from "smart - servier medical art", which provides artwork without licensing (www.smart.servier.com; $12^{\text {th }}$ of August, 2019). 
into the inner hair cell. If a pressure wave now propagates along the cochlea, the basilar membrane (and consequently the hair cells in the organ of Corti) moves with respect to the tectorial membrane, which leads to deflection of the hair cell bundles. Dependent on the direction of movement, these deflections cause an increase or decrease in the mechanical tension on the cation channels. If tension on these channels increases, it causes the channel into a open state, eliciting inward potassium current and thus depolarizing the cell. Vice versa, the probability of channel opening decreases with decreasing tension, reducing potassium influx and hyperpolarizing the cell. Since waves - by nature - are phasic events, the potassium influx and thus the intracellular potential of hair cells oscillates with the phase of the pressure wave moving the basilar membrane, at least for frequencies up to one kHz (Russell and Sellick, 1983). In depolarized phases, voltage gated calcium channels at the base of inner hair cells are opened, leading to calcium influx, which in turn triggers the release of glutamate from the hair cell. The released glutamate subsequently activates afferent fibers of the primary neurons in the auditory system, the spiral ganglion neurons (SGNs), which - if activation is strong enough - initiate action potentials. The somata of the bipolar SGNs are housed in Rosenthal's canal in the modiolus, with their peripheral neurites reaching to the base of inner hair cells and their axons (which together form the auditory nerve) projecting to the cochlear nucleus of the brainstem, thus sending information about signals from the external world to the central nervous system.

\section{The (simplified) neuronal code for sound:}

Waves are characterized by their frequency (i.e. the number of cycles in a given time window) and their amplitude (i.e. the pressure difference of the medium through which waves propagate). In the case of sound waves, the frequency is perceived as pitch, while the amplitude is perceived as loudness. As discussed above, sounds of different frequencies deflect the basilar membrane at different locations, which results in inner hair cell and subsequent spiral ganglion activation at spatially distinct positions in the cochlea ('place code'; figure 2, top vs bottom). The activation of distinct neuronal populations by sound waves of different frequencies is maintained throughout the auditory pathway up to primary auditory cortex - a phenomenon called tonotopy.

Due to this labeled-line feedforward-processing, the brain is able to infer the frequency of an acoustic signal by the spatial pattern of neuronal activity in the auditory system - giving rise to pitch perception. A second mechanism for pitch perception - up to frequencies of $2-4 \mathrm{kHz}-$ is phase locking, where stimulus frequency is not only represented by tonotopy, but also by the timing of neuronal responses: The occurrence of each action potential at a given phase of the sound wave triggering this action potential signals stimulus frequency, since it is identical to the 
frequency of the stimulus ('time code'). However, at higher frequencies (starting from approximately $2-4 \mathrm{kHz}$ ) neurons are not able to follow the stimulation frequency any longer in a phase-locked manner, since they are limited by their maximal firing rates (due to the time constant of the inner hair cell membrane and due to the duration of an action potential and the corresponding refractory time in spiral ganglion neurons). These frequencies are exclusively represented by tonotopy. Regarding the intensity of a sound, it was already mentioned that the deflection of inner hair cell bundles and thus the depolarization of inner hair cells depend on the degree of basilar membrane
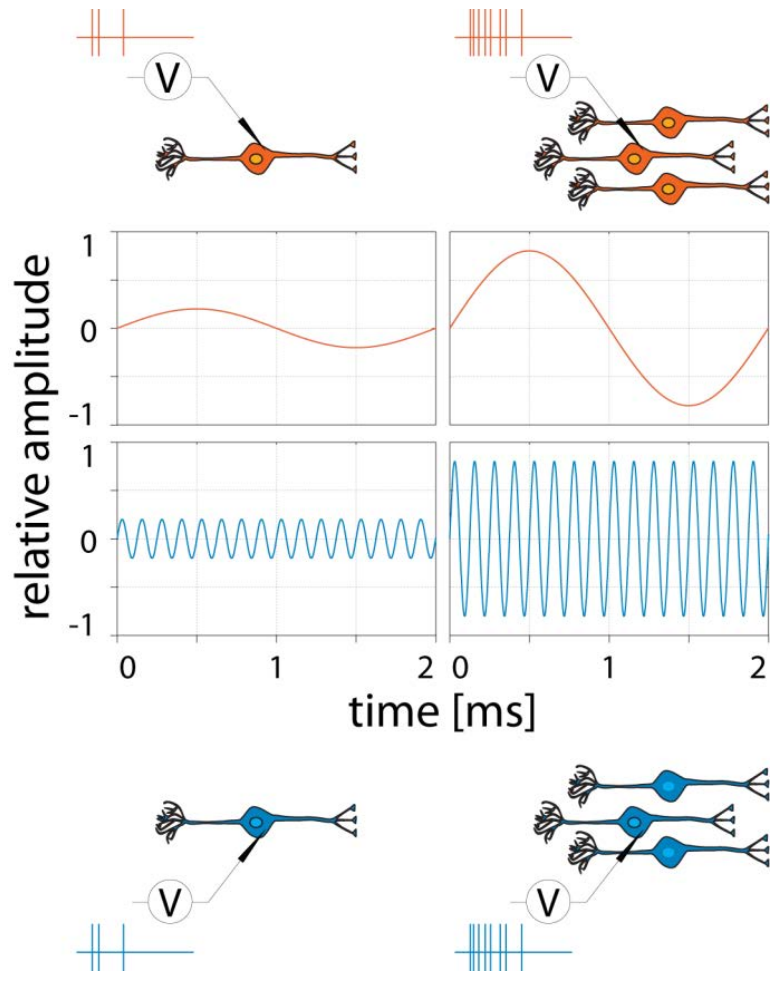

Figure 2: Neural coding for basic features of sound. While sound frequency (top vs bottom) is coded by distinct populations of neurons, amplitude is coded by firing rate in individual neurons and by recruiting higher numbers of neurons on the population level.

displacement: In consequence, waves of higher amplitude cause stronger activation of inner hair cells, more glutamate release and finally the initiation of more action potentials in subsequent spiral ganglion neurons ('rate code'). In addition, waves of higher amplitude displace the basilar membrane over a larger area, resulting in activation of a larger population of inner hair cells and downstream neurons (figure 2, left vs right). Thus, sound intensity is coded by the amount of action potentials in response to a given stimulus on the level of single neurons and by the recruitment of bigger neuronal ensembles on the population level. In contrast to the inner hair cells that drive sound encoding in SGNs, outer hair cells amplify and sharpen sounds in a frequency-specific manner and thus contribute to hearing sensitivity and frequency resolution.

This is of course a very simplified view and most sounds in our environment are complex sounds, composed of several frequencies and intensities. On top, it is also important to detect the location of a sound, and since different sounds in our environment have different meanings, the auditory system is required to discriminate between signal and noise. These features of sound analysis (and some more) are mainly taken care of by the central auditory system. However, for the 
further understanding of this thesis it will be very helpful to mainly remember the above mentioned (basic) mechanisms for coding of sound frequency and intensity.

\section{The central auditory pathway and auditory plasticity:}

Once leaving the cochlea, auditory information is processed in a variety of relay stations which are highly interconnected by both feedforward and feedback connections. Even though auditory processing is still not fully understood, some major pathways have been identified, and the most important one will be described in this section. Auditory information is send to the central nervous system via the auditory nerve (formed by axons of SGNs), which unilaterally project to the ipsilateral cochlear nucleus. While the ventral part of the cochlear nucleus extracts information regarding spike timing and the activation pattern of SGNs, the dorsal part of the cochlear nucleus also receives non-auditory inputs and is involved in relating auditory information to the listener's posture, i.e. position of the head. The main output of the cochlear nucleus is projected from its ventral part to both the ipsi- and the contralateral superior olivary complex, which is the first station to receive binaural information. Differences in time and intensity of this binaural information are used by the superior olive in order to localize sound, before the information is passed to the inferior colliculus (auditory midbrain). In the inferior colliculus, all ipsi- and contralateral ascending pathways converge (even though the contralateral input is dominant) and spatial data from vertical and horizontal planes is integrated. Besides its function in reading out the computation of sound source localization in the olivary complexes, the inferior colliculus is involved in multi-modal sensory reflexes (e.g. startle reflex or vestibulo-ocular reflex) and sensitive to amplitude modulations of acoustic stimuli, playing a major role in speech recognition. Axons of the inferior colliculus then project to the medial geniculate body of the thalamus, which depicts the last subcortical relay of the auditory pathway and is involved in the processing of relative sound intensity and duration. The medial geniculate body is also believed to direct attention towards different auditory stimuli, thus playing a role in discriminating signal from noise. Output from the thalamus is send to the auditory cortex, where the perception of auditory stimuli happens and the listener identifies auditory objects. The auditory cortex is highly interconnected with all kind of auditory as well as non-auditory cortical and subcortical areas and not only perceives auditory objects, but also passes this information to higher brain areas in order to guide attention, integrate multi-sensory information, relate this information to internal processes of the brain such as memory and finally make decisions and initiate (or not initiate) 
adequate motor responses of the listener. Besides the connections to higher brain areas, the auditory cortex has projections to the auditory thalamus (medial geniculate body), midbrain (inferior colliculus), and brainstem (superior olivary complex, cochlear nucleus), which serve the modulation of cochlear sensitivity and the strength of reflexes (Terreros and Delano, 2015). An important feature of the auditory cortex (and to a certain degree also of subcortical auditory structures) is its high degree of plasticity. Due to different mechanisms, the cortex is able to change its properties in response to sound both during development and in mature organisms, and by doing so it is able to ideally use its resources in order to optimize auditory performance (Irvine, 2018). Even though most of plastic changes in the cortex are caused by modulating the excitation-inhibition balance of already existing neuronal connections, cortical plasticity can have many forms and ranges from the timescale of seconds (in the case of stimulus-specific adaptation (Khouri and Nelken, 2015, 2015)) to long-term changes in the case of perceptual learning such as musical training or language development (Irvine, 2018). Not only has plasticity in the auditory cortex been linked to perceptual learning, attention, memory, it also enables the auditory system to counteract and compensate for different forms of hearing impairment - at least to a certain degree (Irvine, 2018). For example, after damaging a given cochlear region, neurons in the tonotopically corresponding location in auditory cortex change their frequency selectivity away from the damaged range of frequencies and instead responded to frequencies coded by adjacent cochlear regions (Robertson and Irvine, 1989). However, plasticity is not always beneficial, as it has for example been linked to tinnitus (Irvine, 2018). In the case of profound hearing loss or deafness, cortical plasticity is utilized for hearing restoration in a different way: Plasticity enables the identification and interpretation not only of natural acoustic stimuli, but also of artificial stimuli, such as electric stimulation of spiral ganglion neurons - a fact that is of particular importance for the function of cochlear implants, which will be discussed later in this thesis (Kral and Sharma, 2012). Due to neuronal plasticity, activity patterns of cochlear implant stimulation which reflect basic principles of sound encoding in the cochlea - can be interpreted sufficiently well to restore speech comprehension. Outcomes in speech understanding of cochlear implant users are typically higher in children implanted during critical periods as compared to adult implantees, and furthermore appropriate training improves speech understanding, both of which are factors contributing to brain plasticity also beyond the auditory system (Kral and Sharma, 2012). 


\section{Auditory pathologies: prevalence and consequences}

As in probably every biological system, failure also happens in the auditory system, and unfortunately it happens with an alarmingly high prevalence: According to the World Health Organization, approximately 466 million people worldwide - which account for $6.1 \%$ of the human population - suffer from disabling hearing loss, resulting in an economic impact of $\sim 750$ billion dollar globally spent on the treatment of hearing loss (www.who.int/deafness/en; $12^{\text {th }}$ of August, 2019). 1 out of 800 children is born with profound hearing impairment, and 15\% of the adult population experience at least mild hearing loss and approximately one third of the population above 65 years of age suffers from hearing impairment (Moser, 2015a; Steel, 2000). While approximately half of the cases of hearing impairment in children can be assigned to genetic mutations, the remaining ones are caused by environmental factors (Moser, 2015a). In addition, acquired hearing loss - mainly noise induced or age related - contributes to make hearing loss the most prevalent sensory disorder. Consequences of hearing loss are diverse and severe: During early childhood, and especially during critical windows of development, the auditory system relies on sensory input in order to establish, mature and maintain the sense of audition (Kral, 2013). Furthermore, the acquisition of vocal speech strongly depends on the auditory system (José et al., 2014). During adulthood, hearing impairments can cause decreased professional capabilities, risk for diseases such as depression, and decreased quality of life in general (Husain et al., 2014; Zinchenko et al., 2018). In elderly, hearing impairment has been linked to cognitive decline and dementia (Shen et al., 2018; Uchida et al., 2019). Furthermore, over all ranges of age, hearing impairment causes social isolation which in turn is linked to manifold subsequent disadvantages.

Hearing loss (or hearing impairment) can generally be classified into three subtypes: conductive, sensorineural and central hearing loss. Conductive hearing loss originates from alterations in the outer or middle ear that hinder the sound wave to reach the cochlea, for example occlusion of the ear canal, perforation of the tympanic membrane or effusion of fluids in the middle ear. It mostly has mild to moderate severity, and is typically successfully treated in otolaryngology by middle ear microsurgery or can be overcome by hearing aids (Dougherty and Kesser, 2015; Lee and Bance, 2018). Central hearing loss originates in the central nervous system and can be caused by lesions in the brainstem, inferior colliculus or auditory cortex (which depicts the minority of cases) or in a more complex manner in combination with various brain diseases. Central hearing loss often co-exists with cognitive decline and possible symptoms include auditory hallucinations, 
neglect, and the inability to filter auditory signals and discriminate speech from noise. Up to date, central hearing loss is the least understood - but fortunately also the least prevalent - form of hearing loss (Gates, 2012; Gates et al., 1996).

Sensorineural hearing loss (SNHL), resulting from dysfunction of the cochlea and/or auditory nerve, depicts the most prevalent form of hearing loss, accounting for approximately $90 \%$ of all cases (Zhang et al., 2018b). SNHL might either be caused by genetic mutations, age, or various external factors, such as physical trauma, noise or ototoxic substances. Since neither hair cells nor SGNs regenerate, SNHL is permanent, and even though various treatment options are currently investigated, they are still far away from application in human patients (Lee and Bance, 2018). Thus, state of the art treatment options for SNHL either aim at the amplification of sound using hearing aids (in mild and moderate cases) or bypass damaged or missing hair cells and directly stimulate spiral ganglion neurons electrically using cochlear implants (in cases of profound hearing loss and complete deafness).

\section{Re-building audition: restoration of sensorineural hearing loss}

As mentioned above, no causal treatment for sensorineural hearing loss is available up to date, and the development of such treatment options is at the preclinical stage. Strategies to treat SNHL depend on the exact pathological mechanism of SNHL and include regenerative approaches as well as gene-therapeutic options. The most promising strategy to treat genetically caused SNHL is gene therapy, where a healthy version of the mutated gene is introduced into the inner ear in order to replace the non-functional version and would allows for protein expression with normal function. However, even though gene therapy to treat SNHL has been demonstrated in mouse models of genetic deafness (Akil et al., 2012; Al-Moyed et al., 2019; Askew et al., 2015; Lentz et al., 2013), there are still major challenges that need to be addressed before considering clinical trials: First and foremost, safe and cell-type specific viruses need to be identified and stable longterm expression of the introduced gene needs to be demonstrated (Moser, 2015a). Second, a safe way to deliver the virus into the anatomically complex inner ear needs to be developed without causing any additional damage to the middle ear, to remaining hair cells in the inner ear or to the vestibular system (Moser, 2015a; Zhang et al., 2018b). Complicating it further, as genetic deafness often goes along with rapid cochlear degeneration, many deafness genes would need to be 
delivered and expressed in early, including prenatal, stages (Chang et al., 2015; Choi et al., 2011; Zhang et al., 2018b). Also, some of the identified deafness genes (for example otoferlin or some usher genes) are very large, making it challenging to pack them in adeno-associated viruses (AAVs; which up to date are the most promising candidates for gene delivery). Strategies to overcome this limitation, including dual-AAV approaches, are investigated, but different viral vectors, such as adenoviruses, offer alternative options (Moser, 2015a). Even if these requirements are met, there are various genes that cause hearing loss. More than 100 of these genes have been identified, and each of them requires the development of a tailor-made therapy, but lots of cases for which genes have not even been identified yet are still remaining (Moser, 2015a). Furthermore, noise induced hearing loss as well as age related hearing loss, which are even more prevalent than genetic defects, would still leave the need for alternative hearing restoration besides gene therapy (Dombrowski et al., 2018).

Similar to a gene-therapeutic approach, where hair cells should be regenerated from supporting cells, one possible alternative to gene therapy is based on drugs: In cases of acquired deafness where hair cells are lost completely, hair cell regeneration could be forced by trans-differentiation of neighboring supporting cells. It has been shown that pharmacological compounds can alter intracellular signaling pathways of cochlear supporting cells and, by doing so, induce transformation of these cells into new hair cells, at least in vitro (Géléoc and Holt, 2014; White et al., 2006). Even though induced transformation of supporting cells into hair cells has not been demonstrated in vivo yet, these findings suggest a possible way of hearing restoration via pharmacological intervention. A second way to treat acquired hearing loss is based on the reintroduction of stem-cell derived hair cells into the cochlea. Towards hair cell replacement, cochlear progenitor cells have been created from embryonic stem cells in vitro and started to express hair cell genes and displayed hair bundles when introduced into the inner ear or develop into sensory epithelia in culture (Koehler et al., 2013; Li et al., 2003; Oshima et al., 2010). Further, it has even been suggested to replace lost spiral ganglion neurons by stem cells: It has been shown that neural progenitors derived from embryonic stem cells, once transplanted into Rosenthal's canal, innervated inner hair cells in the organ of Corti and sent projections to the brainstem (Corrales et al., 2006). A second study confirmed the findings of progenitor differentiation and furthermore demonstrated improved auditory function starting four weeks after transplantation (Chen et al., 2012). Even though these technologies still need to be refined and have their own obstacles, such as integration of SGNs into mature neuronal circuits or proper hair bundle alignment in the case of hair cell replacement, pharmacological interventions and 
stem cell therapies are promising candidates to restore auditory function upon acquired hearing loss resulting from hair cell damage or loss of hair cells or SGNs (Géléoc and Holt, 2014).

\section{Cochlear implants: a success story of neuroprothesis}

Until treatment options such as pharmacological, gene or stem cell therapies will become available for clinical application, which might take decades, cochlear implants (Cls) depict the only option for partial hearing restoration in patients suffering from profound SNHL or deafness (Steel, 2000). In patients with SNHL, Cls bypass the defective transduction from sound to neuronal signals by directly stimulating SGNs with electric current. Basically, a Cl consists of a linear array of stimulation electrodes embedded in silicone, which is placed via the round window in the scala tympani and winds up the cochlea along its tonotopic axis, an internal stimulator, and a corresponding external audio processor. The audio or speech processor picks up signals from the surrounding auditory scene and extracts their most prominent frequency components as well as their relative intensities. Different spectral components are then matched to different electrodes, placed at the tonotopically corresponding regions in the cochlea (Fig. 3A, B). The corresponding intensity in each spectral channel determines the amplitude of electric current that is injected into the cochlea in order to stimulate each region (Lenarz, 2018). Even though excitation of SGNs via electric current is an unnatural mode of stimulation, the brain is able to interpret the resulting SGN activity and decipher auditory information, which is on one hand attributed to the prominent tonotopic organization in the auditory system, and on the other hand to the high degree of brain plasticity and to extensive training of the patients (Kral and Sharma, 2012; Lenarz, 2018; Middlebrooks et al., 2005; Moore and Shannon, 2009). Artificial sound encoding via cochlear implants is effective enough to restore open speech comprehension in most of approximately half a million implanted people, making the cochlear implant the arguably most successful neuroprothesis (Middlebrooks et al., 2005; Moser, 2015b).

However, despite the enormous success of cochlear implants, electrical sound encoding is far away from being perfect and there is an unmet need for improved hearing restoration: When electric current is injected into the cochlea to stimulate SGNs, its spread is relatively large, due to the high content of ions in the electro-conductive environment of the cochlea (Kral et al., 1998). This spread of electric current leads to recruitment of rather large subsets of spiral ganglion 
neurons and - due to their tonotopic arrangement - to rather coarse coding of spectral information (Fig. 3B, Shannon, 1983). The result of this current spread is crosstalk between the individual stimulation channels, which limits the number of independent channels to typically less than ten (Friesen et al., 2001)., as compared to 800-1300 different sound frequencies that regular hearing subjects are able to distinguish (Formby, 1986; Meurmann, 1954) (and even higher values reported from musicians (Liang et al., 2016)). A second limitation of electrical SGN stimulation is the low dynamic range: SGNs respond with an intrinsically low dynamic range (1-2 $\mathrm{dB}$ (Miller et al., 2006)) to electrical stimulation (as compared to $30-40 \mathrm{~dB}$ in response to natural acoustic stimulation (Viemeister, 1988)). Furthermore, large SGN populations are concurrently activated at similar thresholds, limiting intensity coding on the population level. These mechanisms restrict the dynamic range of the electrically stimulated auditory pathway to $10-20$ $\mathrm{dB}$, whereas the normal hearing system can code a dynamic range of up to $120 \mathrm{~dB}$ (Rubinstein, 2004; Zeng, 2004). Ultimately, the restricted frequency and intensity coding of cochlear implants results in limited signal perception, leading to poor speech recognition in background noise and the inability of many users to appreciate music (Friesen et al., 2001; Kohlberg et al., 2014; Zeng and Galvin, 1999). Consequently, the improvement of artificial sound encoding is still under investigation and strategies to improve $\mathrm{eCl}$ outcomes to the users include various approaches: One way towards improvement might be advanced coding strategies, such as dual electrode configurations or multi-polar stimulation (Donaldson et al., 2005; George et al., 2015; Snyder et al., 2004). Another way could be the improvement of the interface between electrodes and neural tissue, which can either be achieved by the use of intra-neural electrodes (Middlebrooks and Snyder, 2007; Pinyon et al., 2014) or by stimulated outgrowth of SGN neurites towards the electrodes (Pinyon et al., 2014). Finally, optical stimulation of SGNs using focused light has been proposed for activation of more confined SGN populations and hence improved frequency resolution (Hernandez et al., 2014; Izzo et al., 2007; Richter et al., 2011). 


\section{Overcoming the bottlenecks: towards optical cochlear implants}

Since light can conveniently be confined in space, it has been suggested to use optical stimulation in order to specifically stimulate small subpopulations of the tonotopically organized spiral ganglion to improve frequency resolution of artificial sound encoding (Fig. 3B, C; 31, 50-52).

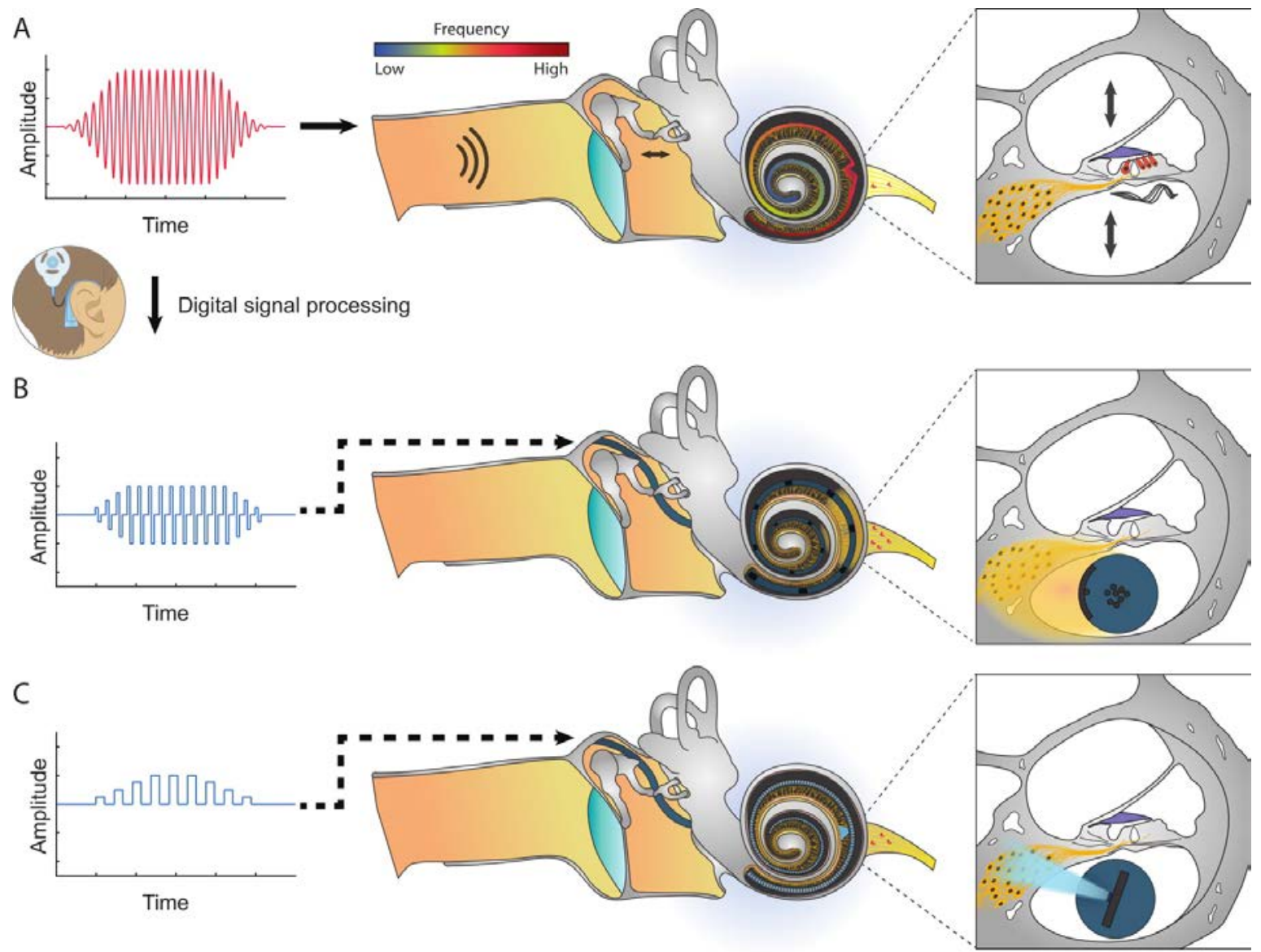

Figure 3: Natural vs artificial sound encoding in the cochlea. (A) Pressure waves in the air (left) are decomposed in a frequency dependent manner (center) and activate mechanosensitive hair cells at the respective cochlear location (right). (B/C) Acoustic signals are analyzed by an external processor which maps the predominant frequencies and corresponding amplitudes of a signal to the stimulator (electrode or optical emitter). Spiral ganglion neurons around the region at which the pressure wave of intracochlear fluids would naturally excite hair cells are then directly activated with electric current (B) or light (C). Since light can be better confined in space than electric current, oCls promise to activate the spiral ganglion with higher spatial selectivity (right, B vs C). This figure was kindly provided by Dr. Daniel Keppeler, Institute of Auditory Neuroscience.

First studies using infrared neural stimulation have demonstrated comparable spatial spread of SGN activation between infrared optical stimulation and acoustic stimulation by in vivo electrophysiology (Richter et al., 2011) and higher spatial confinement of infrared optical stimulation as compared to electrical stimulation by activity-driven gene expression in histological 
analysis of the spiral ganglion (Izzo et al., 2007). While these studies have been very encouraging for the development of optical cochlear implants, the technology of infrared neural stimulation has some major drawbacks: First, the energy requirements for infrared neural stimulation are quite high (typically in the range of tens of Microjoule with a peak illumination of $~ 100 \mathrm{~mW}$; (Matic et al., 2013; Richter et al., 2011)). Second, the mechanism by which infrared stimulation activates neurons is not clear yet and still remains to be clarified (Young et al., 2015). Third - and most important - infrared stimulation of the spiral ganglion neuron could not be confirmed in different studies using animal models of sensorineural hearing loss, which depict the adequate model system for hearing restoration in cochlear implant users (Kallweit et al., 2016; Schultz et al., 2012; Thompson et al., 2015; Verma et al., 2014).

In contrast to infrared neural stimulation, optogenetic activation of Channelrhodopsin (ChR (Nagel et al., 2003)) expressing neurons enables cell-specific excitation via a well-defined molecular mechanism at low light intensities (Boyden et al., 2005). ChRs, which are light-gated ion channels naturally found in algae where they support phototaxis, have been shown to mediate light-gated ion conductance in oocytes of Xenopus laevis one and a half decades ago (Nagel et al., 2002, 2003). Soon thereafter, Channelrhodopsin- 2 has been used to evoke action potentials in mammalian neurons in a fast, non-invasive and cell-type specific manner (Boyden et al., 2005). Since this milestone, the optogenetic toolbox has been expanded tremendously by using directed mutagenesis of already known ChRs as well as large-scale screening of naturally occurring opsins: The variety of available optogenetic tools now includes light gated ion-channels and -pumps (Shevchenko et al., 2017; Yizhar et al., 2011) both for neural excitation and inhibition (Wietek et al., 2014; Yizhar et al., 2011), with time constants ranging from milliseconds to minutes (Berndt et al., 2008; Klapoetke et al., 2014; Yizhar et al., 2011), characterized by faster kinetics (Gunaydin et al., 2010; Klapoetke et al., 2014; Mager et al., 2018), increased light sensitivity of neurons (Kleinlogel et al., 2011), and shifted peak action spectra (Klapoetke et al., 2014; Mager et al., 2018; Yizhar et al., 2011). Thus, optogenetic techniques for precise neural control not only have rapidly gained great importance in neuroscientific research (Fenno et al., 2011; Knöpfel et al., 2010), but furthermore raise hope to be applied for innovative therapies of disorders such as Parkinson's disease (Delbeke et al., 2017), cardiac dysfunction (Schneider-Warme, 2018), epilepsy (Tønnesen and Kokaia, 2017), and sensory disorders such as blindness (Scholl et al., 2016) and deafness (Dombrowski et al., 2018). 
In the past years considerable progress has been made towards optogenetic hearing restoration: In a first proof of principle study, the feasibility of optogenetic activation of the auditory pathway has been demonstrated in transgenic mice broadly expressing Channelrhodopsin- 2 under the Thy1 promotor (Arenkiel et al., 2007) by recordings of optically evoked auditory brainstem responses (OABRs), recordings of action potentials in single auditory nerve fibers, and recordings of local field potentials in the inferior colliculus (Hernandez et al., 2014). Indeed, current source density analysis revealed a smaller spread of excitation upon optogenetic stimulation as compared to monopolar electrical stimulation. Furthermore, cochlear optogenetics could restore auditory activity in deaf mice (Hernandez et al., 2014). Follow-up studies were performed in mice following virus injection into the cochlea during the first postnatal week in order to express ChRvariants with faster gating kinetics in spiral ganglion neurons: In one study, injections of AAV2/6 carrying the ChR-mutant f-Chrimson under the human synapsin promotor (hSyn) led to homogeneous opsin expression in SGNs across all cochlear turns, with a mean transfection rate of 80\% (Mager et al., 2018). F-Chrimson, an opsin with a red-shifted action spectrum and faster closing kinetics $\left(\tau_{\text {off }}=3.2 \mathrm{~ms}\right.$ ), enabled optogenetic stimulation of SGNs with good temporal fidelity up to several hundred Hertz at low light intensity thresholds ( $0.5 \mu \mathrm{J})$, demonstrated by recordings of OABRs and activity of individual auditory nerve fibers (Mager et al., 2018). Opsinmediated activation of SGNs could furthermore restore activity in the auditory pathway (Mager et al., 2018) of a mouse model of age-related hearing loss (Shnerson et al., 1981). In a second study, a viral construct mediating neural expression of the blue-light activated ChR-variant Chronos has been optimized for the auditory system (Keppeler et al., 2018): Chronos (Klapoetke et al., 2014), the fastest naturally occurring opsin known today $\left(\tau_{\text {off }}<1 \mathrm{~ms}\right)$, was combined with an export signal for improved endoplasmic reticulum export (ES; taken from inward rectifying potassium channels (Kir2.1) (Gradinaru et al., 2010; Hofherr, 2005; Ma et al., 2001)), a trafficking signal for improved membrane localization (TS; also taken from channels of the Kir2.x family (Gradinaru et al., 2010; Stockklausner et al., 2001)), and packaged in the recently engineered AAV-PHP.B for enhanced viral transduction (Deverman et al., 2016). Early postnatal injection of the AAV-PHP.B-ChronosES/TS construct in the mouse cochlea enabled highly efficient Chronos-expression in spiral ganglion neurons, OABRs at thresholds of $5 \mu \mathrm{J}$ and $100 \mu$ s per pulse, and spiking of individual SGNs up to several hundred Hertz repetition rate (Keppeler et al., 2018). Together, these studies demonstrate that a major requirement for optogenetic hearing restoration - efficient transduction of auditory neurons with opsins mediating light-induced generation of action potentials at firing rates that approach the physiological rates observed in SGNs - can be met by tailored optogenetics. 
Besides the biological feasibility of light-based SGN-activation, a second prerequisite for optogenetic hearing restoration concerns the technological site: biocompatible multi-channel optical stimulators, small and flexible enough to allow for cochlear implantation and stable enough to work over decades, must be developed to facilitate excitation of the optogenetically manipulated auditory nerve. Also towards this end considerable work has been done in the past years: One study demonstrated the fabrication of gallium-nitride (GaN) based light emitting diodes in the micrometer range ( $\mu$ LEDs) which are linearly arranged on a flexible substrate based on polyimide and silicon (Goßler et al., 2014). In this study, an optical cochlear implant (oCl) housing $15 \mu$ LEDs with a size of $150 \times 150 \mu \mathrm{m}$ (from which up to three $\mu$ LEDs could be addressed separately) was successfully inserted into the model of a rat cochlea. A second implant with four linearly arranged $\mu$ LEDs $(50 \times 50 \mu \mathrm{m})$ was implanted into a mouse cochlea via the round window. Driven by a current of $1 \mathrm{~mA}$, the output power of these four $\mu$ LEDs at a wavelength of $405 \mathrm{~nm}$ was $60 \mu \mathrm{W}$, corresponding to an optical power density of $6 \mathrm{~mW} / \mathrm{mm}^{2}$ (Goßler et al., 2014). Following up on this work, an epoxy-based, biocompatible and translucent $\mathrm{oCl}, 350 \mu \mathrm{m}$ wide and $1.5 \mathrm{~cm}$ long, housing a total of $144 \mu \mathrm{LEDs}$ which can be addressed in a matrix, has recently been realized (Klein et al., 2018). Besides the enormous increase in the number of individual light emitters, the presented $\mathrm{oCl}$ showed a reduction in thermomechanical bending as compared to the previous implants and a maximum temperature increase of $1{ }^{\circ} \mathrm{C}$ at driving currents up to $10 \mathrm{~mA}$, which are further requirements for safe in vivo application. Finally, the optical power (at a wavelength of $462 \mathrm{~nm}$ ) has been increased by almost two orders of magnitude to $0.82 \mathrm{~mW}$ (when driving the implant with $10 \mathrm{~mA}$ ), corresponding to a power density of $407 \mathrm{~mW} / \mathrm{mm}^{2}$ (Klein et al., 2018). Even though functionality and stability of optical cochlear implants in vivo remains to be demonstrated, these studies proof the general feasibility of industrial oCl fabrication at the level of wafers which fulfill basic requirements of potential oCls regarding size, number of emitters and light output while considering thermomechanical properties such as flexibility and heating of the implants. Together, the biomedical and technological developments towards oCls during the past years raise great hope that the mayor limitations of nowadays $\mathrm{Cls}$ might be overcome and the quality of artificial hearing for deaf patients might be improved in the future. 


\section{Scope of this thesis: validating cochlear optogenetics in adult Mongolian gerbils}

Even though tremendous work towards the development of optical cochlear implants has been done in the past years, a long way remains to be gone before considering the translation of oCls towards clinical application. While Chronos and f-Chrimson are promising candidates as molecular tools to mediate cochlear optogenetics, a reliable method for neural transduction, also in the adult organism, needs to be established. Not only must this transduction be efficient and longlasting, but it must also be specific, ideally involving the use of SGN specific promotors. Having the molecular requirements checked, the technological development of stable and functional oCls must be realized - not only for the light-emitting intracochlear part of the implant, but also for the oCl processor, including a coding strategy to transform real world sounds into optical signals while minimizing the energy requirements for oCl coding. In a next step, these technologies must be verified in preclinical studies using appropriate animal models. First and foremost, functionality of oCls must be demonstrated: Neural activation of SGNs as well as subsequent signal propagation along the auditory pathway must be characterized and behaviorally relevant perception of these stimuli must be demonstrated, ideally during the whole lifespan of the model organism and in animal models of sensorineural hearing loss. Second, the benefit of oCls compared to eCls must be demonstrated. Only if fundamental improvement of this technology over the limitations of electrical $\mathrm{Cls}$ is expected, the clinical translation of cochlear optogenetics can be justified. Third, the technology must be translated to a non-human primate model in order to confirm that the results obtained from rodent work are also valid in primates. Finally, biosafety studies must be conducted and all necessary tools to realize optical cochlear implants must be optimized for application in humans. Out of these challenges that need to be addressed towards the development of an optogenetic cochlear implant, this thesis deals with three major tasks:

\section{Perception of cochlear optogenetics and optogenetic hearing restoration:}

One basic requirement for optogenetic sound encoding is the perception of optogenetic SGN stimulation. Towards this end, a fiber-based single channel oCl has been implanted via the round window into the cochlea of adult Mongolian gerbils, in which SGNs have been optogenetically transduced by intramodiolar injections of AAV2/6 carrying a plasmid encoding the Calcium Translocating ChR2- CatCh. Light-evoked auditory brainstem responses have then been recorded over the following weeks, in some animals up to four months after implantation, to verify optogenetic SGN activation on a physiological level. In parallel, these animals have been trained in a behavioral task using the shuttlebox paradigm to indicate perception of optogenetic SGN 
stimulation via avoidance behavior. Avoidance behavior was subsequently also triggered by using acoustic stimulation, suggesting at least some degree of similarity between the perception of acoustic and optogenetic stimuli. After training the animals in the task, thresholds for intensity and duration of light pulses have been determined to estimate the energy requirements of oCl coding. Finally, optogenetic SGN stimulation has been shown to restore activation of the auditory system both on a physiological and behavioral level in a gerbil model of sensorineural hearing loss, where inner hair cells have been lost upon intracochlear kanamycin application.

\section{Comparison of spectral selectivity upon optogenetic and electrical SGN stimulation:}

To justify the development of oCls, an increase in spectral resolution as compared to eCls - which are currently used in clinical settings - must be demonstrated. Towards this end, SGNs of optogenetically modified, isoflurane-anesthetized Mongolian gerbils have been stimulated via optical fibers at three distinct positions in the cochlea, while multi-channel electrophysiological recordings have been performed in the tonotopically layered inferior colliculus. As a comparison, SGNs of wildtype gerbils have been stimulated either acoustically or electrically via clinical-style 4channel eCls. Using an activity-based analysis of neural activation in the inferior colliculus, the spread of cochlear excitation upon neural stimulation with the different modalities could be estimated: It was shown that the spectral selectivity of optogenetic SGN stimulation is comparable to acoustic stimulation using pure tones at low stimulus intensities. Furthermore, optogenetic stimulation was spectrally more selective than bipolar electrical stimulation at modest and high stimulus intensities, while it outperformed monopolar electrical stimulation at all stimulus intensities. Thus, an increase in spectral selectivity of oCl versus $\mathrm{eCl}$ coding has been demonstrated, suggesting that optical cochlear implants based on optogenetics might overcome the major bottleneck of eCls.

\section{In vivo validation of $\mu L E D$ based optical cochlear implants:}

Towards the technical development of optical cochlear implants, functionality of $\mu$ LED-based 16channel oCls has been demonstrated in optogenetically modified Mongolian gerbils. Multichannel electrophysiological recordings have been performed in the inferior colliculus of isoflurane-anesthetized gerbils, while SGNs have been stimulated optically by either driving individual $\mu L E D s$, groups of four neighboring $\mu L E D s$, or all $16 \mu L E D$ of the $\mathrm{OCl}$ at a time. It was shown that individual $\mu$ LEDs of the $\mathrm{OCl}$ were able to evoke neural responses in the inferior colliculus, and that the strength of neural responses increased when recruiting additional $\mu$ LEDs. The spread of excitation upon $\mu L E D$-based oCl stimulation - even when stimulating with groups of 
four subsequent $\mu$ LEDs - was shown to be more specific than the spread of excitation upon electrical SGN stimulation. Taken together, this study demonstrates the feasibility of SGN activation by $\mu L E D$-based oCls with increased frequency resolution as compared to eCls.

In conclusion, the results of this thesis demonstrate the perception of optogenetic SGN stimulation with reasonable energy requirements in a gerbil model of sensorineural hearing loss. Furthermore, the increased frequency selectivity of optical over electrical $\mathrm{Cls}$ has been demonstrated using optogenetic stimulation via optical fibers. Finally, in vivo functionality of a $\mu L E D$-based, 16-channel optical $\mathrm{Cl}$ has been demonstrated. Taken together, these results raise big hopes that optogenetic hearing restoration might overcome the major bottleneck of electrical hearing restoration and thus improve the quality of artificial sound encoding for future patients. 


\section{Chapter I: Optogenetic Stimulation of cochlear neurons activates the auditory pathway and restores auditory driven behavior in deaf adult gerbils}

\footnotetext{
Christian Wrobel ${ }^{1,2,3, \#, \$}$, Alexander Dieter ${ }^{1,4,5, \#}$, Antoine Huet ${ }^{1,5, \S}$, Daniel Keppeler ${ }^{1,4, \S}$, Carlos J. DuqueAfonso $^{1,4}$, Christian Vogl ${ }^{2,6}$, Gerhard Hoch ${ }^{1,2,5}$, Marcus Jeschke ${ }^{1,5,{ }^{*}}$, Tobias Moser ${ }^{1,2,4,5,7,8, *}$

${ }^{1}$ Institute for Auditory Neuroscience and InnerEarLab, University Medical Center Göttingen, 37075 Göttingen, Germany.

${ }^{2}$ Collaborative Research Center 889, University of Göttingen, 37075 Göttingen, Germany.

${ }^{3}$ Department of Otorhinolaryngology, Head and Neck Surgery, Ruhr University Bochum, St. Elisabeth Hospital, 44787 Bochum, Germany.

${ }^{4}$ Göttingen Graduate School for Neurosciences and Molecular Biosciences, University of Göttingen, 37075 Göttingen, Germany.

${ }^{5}$ Auditory Neuroscience and Optogenetics Group, German Primate Center, 37077 Göttingen, Germany

${ }^{6}$ Presynaptogenesis and Intracellular Transport in Hair Cells Group, Institute for Auditory Neuroscience and InnerEarLab, University Medical Center Göttingen, 37075 Göttingen, Germany

${ }^{7}$ Bernstein Center for Computational Neuroscience, University of Göttingen, 37077 Göttingen, Germany

${ }^{8}$ Center for Nanoscale Microscopy and Molecular Physiology of the Brain, University of Göttingen, 37075 Göttingen, Germany

$\#, \S$ equal contribution
}

\$ current affiliation: Department of Otorhinolaryngology and InnerEarLab, University Medical Center Göttingen, 37075 Göttingen, Germany.

*To whom correspondence should be addressed:

Dr. Marcus Jeschke, Email: marcus.jeschke@med.uni-goettingen.de

Auditory Neuroscience and Optogenetics Laboratory, German Primate Center, Kellnerweg 4, 37077 Göttingen

Dr. Tobias Moser, Email: tmoser@gwdg.de

Institute for Auditory Neuroscience and InnerEarLab, University Medical Center Göttingen, Robert-Koch-Straße 40, 37075 Göttingen

Copyright notice:

From: C. Wrobel, A. Dieter, A. Huet, D. Keppeler, C. J. Duque-Afonso, C. Vogl, G. Hoch, M. Jeschke, T. Moser, Optogenetic stimulation of cochlear neurons activates the auditory pathway and restores auditory-driven behavior in deaf adult gerbils. Sci. Transl. Med. 10, eaao0540 (2018). Reprinted with permissions from AAAS.

DOI: 10.1126/scitransImed.aao0540

Copyright (C) 2018 The Authors, some rights reserved; exclusive licensee American Association for the Advancement of Science. 


\section{Author contributions:}

C.W., A.D., M.J., and T.M. designed the study. C.W. performed AAV injections, immunohistochemistry, OABR/AABR recordings, and SGN recordings in the early phase of the project. C.W and M.J. performed recordings from single AC units. A.D. performed AAV injections, chronic oCl, oABR/aABR recordings, shuttlebox, and deafening experiments. A.D. and M.J. designed behavioral experiments. A.H. performed AAV injections and recordings from single SGNs that are presented in this manuscript. D.K. performed x-ray tomography. C.J.D.-A. performed the Monte Carlo modeling of optical rays. C.V. performed the immunohistochemistry of whole-mount organs of Corti. G.H. and M.J. designed the shuttlebox and other hard/software. All authors analyzed data and prepared the manuscript. Competing interests: The authors declare that they have no competing interests.

\section{Detailed author contributions of Alexander Dieter (A.D.):}

\section{Conceptual work:}

- Contribution to experimental design of fiber implantation, behavioral experiments, and hearing restoration, initially layouted by Prof. Dr. T. Moser and Dr. M. Jeschke

Experimental work:

- Intramodiolar virus injections in adult Mongolian gerbils ( $40-50 \%$ of the animals used for this manuscript)

- Recording of acoustically and optogenetically evoked auditory brainstem responses ( $50 \%$ of the ABR data presented throughout the manuscript)

- Establishing and performing fiber implantations in the cochlea for chronic optical stimulation of the auditory nerve (all chronic data presented in the manuscript)

- Establishing and performing behavioral experiments in the shuttle box (all behavioral data presented in the manuscript)

- Pharmacological deafening and fiber implantation for optogenetic hearing restoration, auditory brainstem responses and behavioral experiments (all restoration data presented in this manuscript)

Data analysis:

- Analysis of auditory brainstem responses ( $\sim 50 \%$ of the data presented in the manuscript, with the use of a toolbox developed by Dr. Christian Wrobel)

- Development of Matlab-Code to analyze behavioral experiments as well as analysis of all behavioral data presented in this manuscript

Data presentation:

- First draft and editing of the material and methods as well as results for the experiments performed by Alexander Dieter, discussions and editing for the rest of the manuscript

- Preparation of figures 4, 5 and 7 displayed in the manuscript and figures 4, 6 and 10 displayed in the supplementary information 


\title{
One Sentence Summary
}

Virus-mediated expression of the calcium translocating channelrhodopsin (CatCh) in cochlear neurons of adult gerbils enables optogenetic stimulation of the auditory pathway and restores auditory driven behavior.

\begin{abstract}
Cochlear implants (Cls) partially restore hearing via direct electrical stimulation of spiral ganglion neurons (SGNs). However, spread of excitation from each electrode limits spectral coding. Here we explored the use of optogenetics to deliver spatially-restricted and cell-specific excitation in the cochlea of adult Mongolian gerbils. We characterized the light-induced activity in the auditory pathway by electrophysiological and behavioral analysis. Modiolar injection of adeno-associated virus (AAV) coding for the light-sensitive calcium translocating channelrhodopsin (CatCh) in adult gerbils successfully infected SGNs of all cochlear turns. SGNs showed stable and long lasting CatCh expression and electrophysiological recording from single SGNs showed that light stimulation up to few hundred Hertz $(\mathrm{Hz})$ induced neuronal firing. Moreover, light-induced optical auditory brainstem responses ( $O A B R s$ ) were comparable to normal acoustic $A B R$ ( $A A B R$ ). In normal hearing animals, chronic optical $\mathrm{Cl}(\mathrm{OCl})$ elicited stable oABRs over weeks. Light stimulation was able to induce cued avoidance behavior that was transferred to acoustic stimulation. Neurons of the primary auditory cortex responded with change in firing rates with increasing light power. In adult deafened gerbils, light stimulation generated $O A B R$ and induced cued avoidance behavior indicating partial restoration of auditory function. In conclusion, our data show that optogenetic sound encoding is feasible with low thresholds and high temporal fidelity in an adult rodent model, suggesting that optogenetics might be used to develop $\mathrm{Cl}$ with improved restorative capabilities.
\end{abstract}




\section{Introduction}

Approximately 360 million people suffer from disabling hearing impairment $(\mathrm{HI})(1) \mathrm{HI}$ hampers communication and often causes social isolation, depression and reduction in professional capabilities. Sensorineural $\mathrm{HI}$ is the most common form of hearing loss; it results from cochlear dysfunction or degeneration typically involving loss of sensory hair cells. Hearing aids and electrical cochlear implants (eCls) provide partial restoration of hearing for sensorineural HI. The eCl bypasses dysfunctional or lost cochlear hair cells via direct electric stimulation of SGNs and provides most of the approximately 500.000 users with open speech comprehension (2-4). One of the goals of current $\mathrm{Cl}$ research is to increase the coding of spectral information that is very limited in eCls because of wide spread of current around each electrode contact (5). The resulting channel-crosstalk limits the number of useful frequency channels to less than ten and explains why eCl users suffer from poor speech comprehension in noisy environments. Increasing the frequency resolution of coding has been explored using multipolar stimulation, intra-neural electrodes, engineered outgrowth of neurites towards $\mathrm{eCl}$ contacts and stimulation by optical $\mathrm{Cl}$ (oCl) (6-12).

A first proof-of-principle study on optogenetic SGN-stimulation used transgenic mice and rats as well as prenatal adeno-associated virus (AAV) mediated gene transfer to mouse SGNs and demonstrated activation of the auditory pathway up to the inferior colliculus and a lower spread of cochlear excitation for fiber-based $\mathrm{oCl}$ than for monopolar eCl (12). However, much remains to be done in order to further develop, characterize and optimize oCls on their way towards potential clinical translation. Critically, a postnatal approach for manipulating SGNs across all cochlear turns needs to be established. Moreover, although oCl have been shown to improve frequency selectivity compared to $\mathrm{eCl}$, this seemed to be traded in for poorer temporal fidelity of oCl coding (12). Higher temporal fidelity of oCl coding might be achieved by using faster channelrhodopsins (ChRs) such as Chronos, which has already been tested for optogenetic stimulation of the auditory brainstem, or the recently developed ChR2 variant CatCh. CatCh seems to support rapid repolarization due to enhanced $\mathrm{Ca}^{2+}$ influx recruiting more largeconductance $\mathrm{Ca}^{2+}$-activated $\mathrm{K}^{+}$-channels and might thus be of interest for oCl coding (13-16). Finally, although mice and rats are often the species of choice in preclinical studies, testing $\mathrm{oCl}$ in other species with a hearing system more closely resembling human condition is critical to understand the translational potential of oCls. 
Here, we established AAV-mediated expression of CatCh in SGNs of adult Mongolian gerbils and characterized optogenetic stimulation by electrophysiology and behavioral analysis. The gerbil is of particular interest for preclinical studies of oCl, because in contrast to other rodents, its hearing extends to the low frequency range used by the human ear and its cochlea is relatively large (only approximately 2.5 -fold smaller than the human cochlea). We demonstrate that optogeneticallydriven activity achieves good temporal fidelity. Using chronic oCls we show the reliability of optogenetic stimulation over weeks and find that it potently cues avoidance behavior. We characterize the response of single neurons of the auditory cortex to oCl stimulation. Finally, we show that oCl restores some auditory function in a gerbil model of ototoxic deafness. 


\section{Results}

Optogenetic manipulation of the adult spiral ganglion via direct modiolar AAV-CatCh-injection: We first tested the transfection properties of Chronos and CatCh using various administration routes in adult gerbils (fig. S1A). Compared to the strong abundance of CatCh in the plasma membrane of SGNs, Chronos showed a more intracellular expression pattern and did not evoke oABRs (fig. S1B, C); therefore we decided to use AAV2/6 carrying CatCh linked to the reporter protein enhancedYellow-Fluorescent-Protein (eYFP) under control of the human synapsin (hSyn) promoter for injections into the modiolus of adult gerbils (8-19 weeks of age) (Fig. 1A). We adapted a retroauricular approach previously used to graft neural precursor cells (17). We approached the spiral ganglion from the bulla using a fine dental file bypassing the scala tympani to inject 2-3 $\mu \mathrm{l}$ of AAV-CatCh-suspension (Fig. 1B-D).

The number of SGNs and their expression of CatCh-eYFP were analyzed by confocal microscopy of immunolabeled mid-modiolar cryo-sections 4-12 weeks after injection in animals that showed functional response to optical stimulation (46\%, 44 out of 96 injected animals). CatCh-eYFP expression was limited to SGNs of the injected ear, which showed prominent labeling in the plasma membrane of somata and neurites all the way to the inner hair cells (IHCs, Fig. 1E). SGNs of all three cochlear turns (apex, mid and base) were transduced to a similar extent (on average approximately $30 \%$, Fig. 1F). A mild loss of SGNs across all turns was observed in the injected cochlea (approximately $25 \%$, significant in comparison with the right non-injected side, $p<0.001$, Fig. 1G). A similar extent of SGN loss was also found in AAV-CatCh-injected ears that largely lacked CatCh-expression (approximately $35 \%$, significant in comparison to the right non-injected side, $\mathrm{p}$ $=0.006)$, suggesting that the SGN loss likely resulted from damage caused by the pressure injection into the fixed volume of Rosenthal's canal housing the SGNs (fig. S2). 

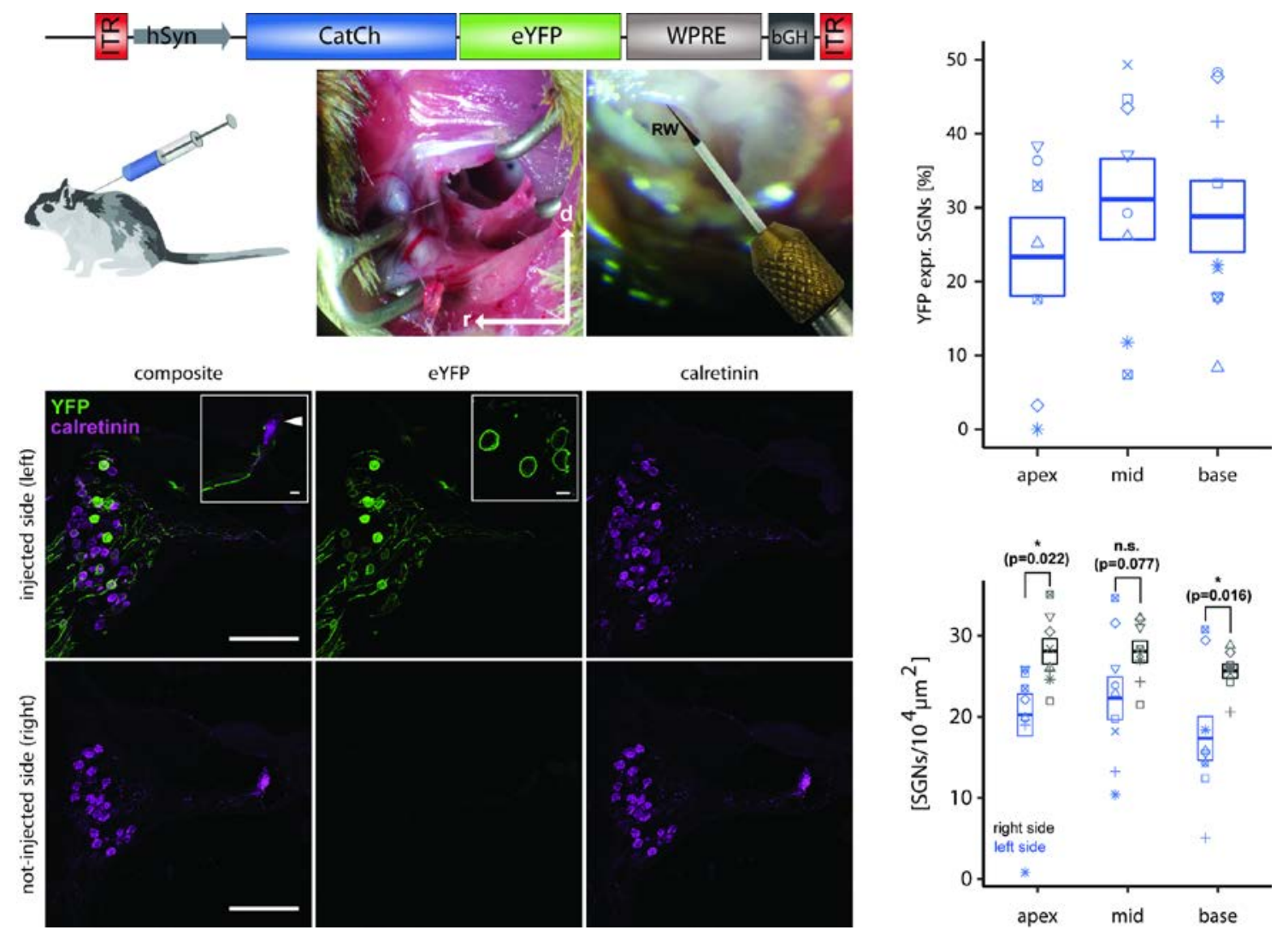

Figure 1. AAV-CatCh-mediated optogenetic manipulation of cochlear SGNs in adult gerbils. (A) Scheme of the AAVconstruct used to transduce SGNs with CatCh-eYFP. (B) Depiction of retroauricular injection of the AAV-CatCh. (C) Photo of the retroauricular approach to the middle ear of a gerbil $(r=$ rostral, $d=$ dorsal): the bullostomy provides a view into the middle ear. (D) Montage of images showing the round window (RW) niche and dye-filled glass capillary, which points to the manually drilled hole. (E) Confocal images of immunolabeled mid-modiolar cochlear cryosections (representative section of a middle turn) of an AAV-CatCh injected adult gerbil. eYFP (green) marks transduced SGNs, calretinin (magenta) generically marks SGNs, scale bar: $100 \mu \mathrm{m}$. It should be noted that the apparent abundance of CatCh in the cytoplasm results from the maximum projection as individual z-sections demonstrate a clear membrane expression. Inset (upper left): calretinin-positive but eYFP-negative inner hair cell (arrowhead) with associated eYFPpositive, peripheral SGN neurites, scale bar: $10 \mu \mathrm{m}$. Inset (upper center): Close-up single z-section of SGNs, highlighting the apparent plasma membrane expression of the construct. Scale bar: $10 \mu \mathrm{m}$. (F) Box plot showing fraction of eYFP expressing SGNs for the apical, middle and basal cochlear turn of the injected left ear. No expression was found in the non-injected right ear $(n=9)$. (G) Box plot showing SGN density for the apical, middle and basal cochlear turn of the injected left ear and the non-injected right ear $\left(n=9,\left(^{*}\right): p<0.05\right.$ [t-test]). Data in $F$ and $G$ are shown as mean \pm SEM.

Characterization of optical activation of the auditory pathway by auditory brainstem responses: To characterize the light-induced activation of the auditory pathway, we first recorded oABRs using fiber-coupled laser stimulation 4-12 weeks after AAV-CatCh-injection (Fig. 2A). Blue light stimulation through a cochleostomy in the middle cochlear turn or through the round window evoked oABRs in AAV-CatCh-injected animals (Fig. 2B, fig. S3). Optical stimulation induced one to four peaks in the $A B R$, which likely reflected the synchronous activation of CatCh-expressing SGNs (first peak) and the downstream auditory pathway (subsequent peaks). OABR amplitudes varied between animals and were positively correlated with the fraction of CatCh-expressing SGNs (fig. 
S4A, B). Increasing light intensity resulted in increased $O A B R$ amplitude (quantified by the difference between the first positive [p1] and negative peak [n1]) and decreased the latency to response (Fig. 2C, D; quantification for the second positive and negative peak [p2-n2] is shown in fig. S4C, D). Even though the average threshold of acutely measured oABRs amounted to $4.6 \mathrm{~mW}$ $( \pm 2.8 \mathrm{~mW}, \mathrm{n}=14$ ), stimuli as weak as $1-2 \mathrm{~mW}$ (duration: $1 \mathrm{~ms}$, rate: $10 \mathrm{~Hz}, 1-2 \mu \mathrm{J}$ per pulse) were sufficient to drive oABRs in some animals. In 8 out of 20 animals, increasing the light intensity increased $O A B R$ amplitudes over more than one order of magnitude the response did not plateau in the majority of animals ( $75 \%$ without a plateau) such that we could only approximate an apparent dynamic range from the stimulus-amplitude functions, which amounted to $16.02 \pm 6.14$ $\mathrm{dB}$. The latency of the first $O A B R$ peak was $0.95 \pm 0.17 \mathrm{~ms}$. Analyzing $O A B R$ in response to different stimulus durations we found that oABRs could be elicited by stimuli as short as $200 \mu \mathrm{s}$ (fig. S4E, F).

Analysis of the dependence of oABRs on the stimulus rate (duration: $1 \mathrm{~ms}$, intensity: $21-32 \mathrm{~mW}$ ) showed that increasing stimulus rate reduced OABR amplitude and prolonged latency; however, oABRs remained sizable up to stimulus rates of at least $200 \mathrm{~Hz}$ (Fig. 2E-G, fig. S4G, H).

Quantitative comparison of acoustically-evoked $A B R(a A B R)$ from unrelated, non-injected gerbils and $O A B R$ data with the highest laser intensity possible at our setup ( $\sim 30 \mathrm{~mW}$ ) showed similar amplitudes with clicks of $40 \mathrm{~dB}$ peak equivalent sound pressure level (SPL), while larger amplitudes of aABRs evoked by clicks of 50-70 dB exceeded those of oABRs (Fig. 2H-J, fig. S5A). Latencies of oABRs were significantly shorter than for aABRs evoked by clicks even at $80 \mathrm{~dB}$ SPL ( $p$ $<0.0001$, fig. S5B).

P1-n1-amplitude declined and p1-latency increased with increasing stimulation rate for both oABR and aABR (Fig. 2L, M). To evaluate the magnitude of the effect of stimulation rate on $A B R$ amplitude, we measured the p1-n1-amplitude ratio measured at $150 \mathrm{~Hz}$ over $10 \mathrm{~Hz}$ stimulation rate. For $\mathrm{aABR}$ the ratio amounted to $0.39 \pm 0.15$, indicating a 2.5 -fold reduction (mean $\pm S D$, $\mathrm{n}=13$ gerbils). For $\mathrm{OABR}$, the ratio was $0.67 \pm 0.37$, indicating a 1.5 -fold reduction (mean $\pm S D$, $\mathrm{n}=20$ gerbils). Altogether these results suggest that the CatCh-mediated optogenetic coding achieves a temporal fidelity similar to that of acoustic coding. 
A
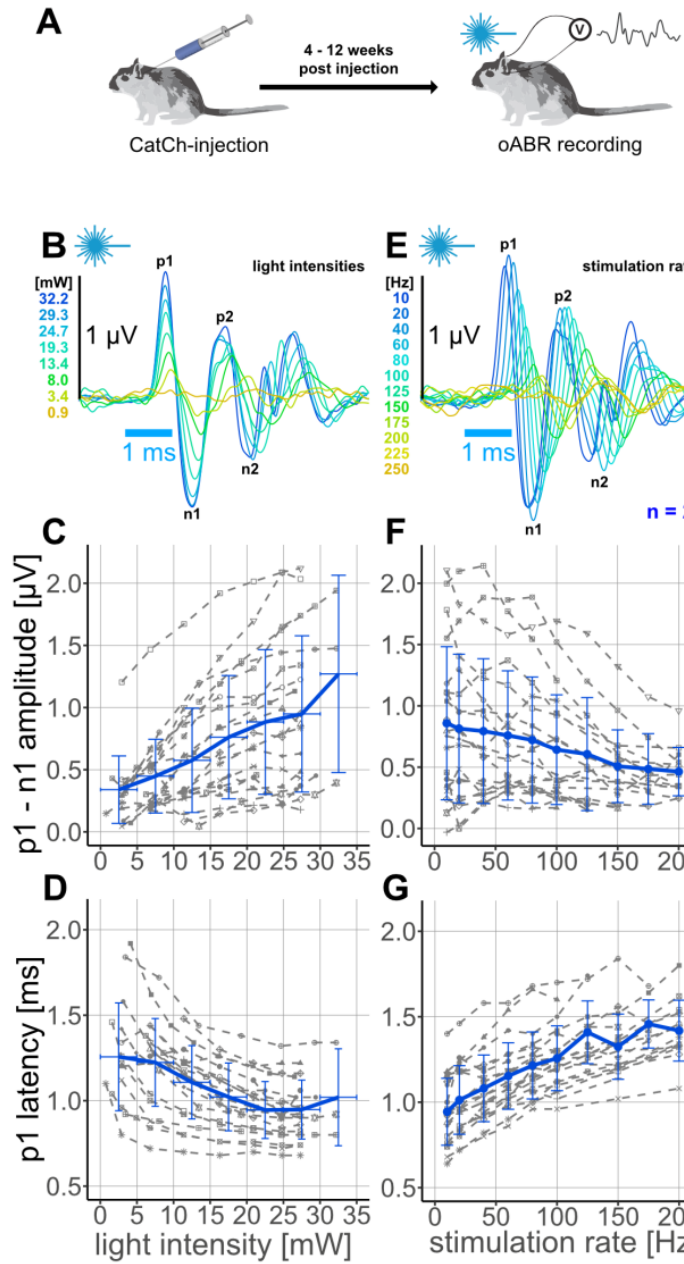
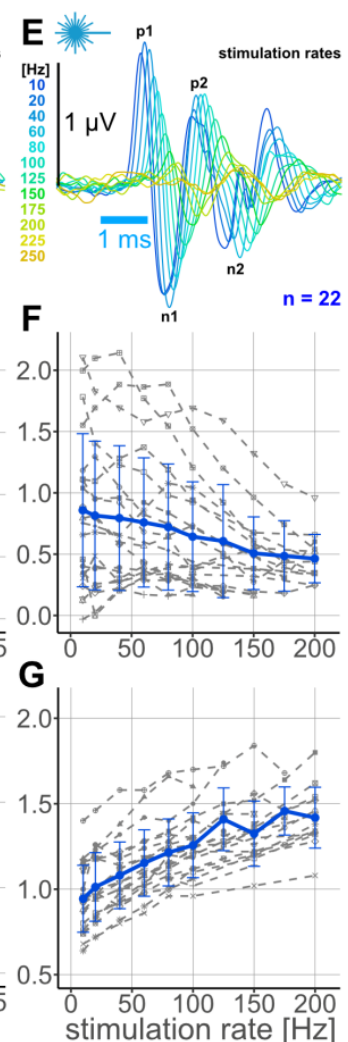
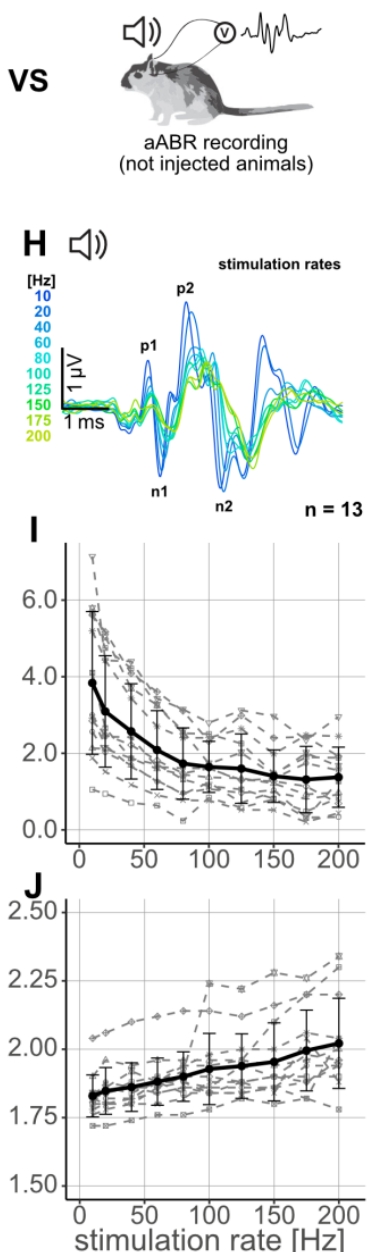
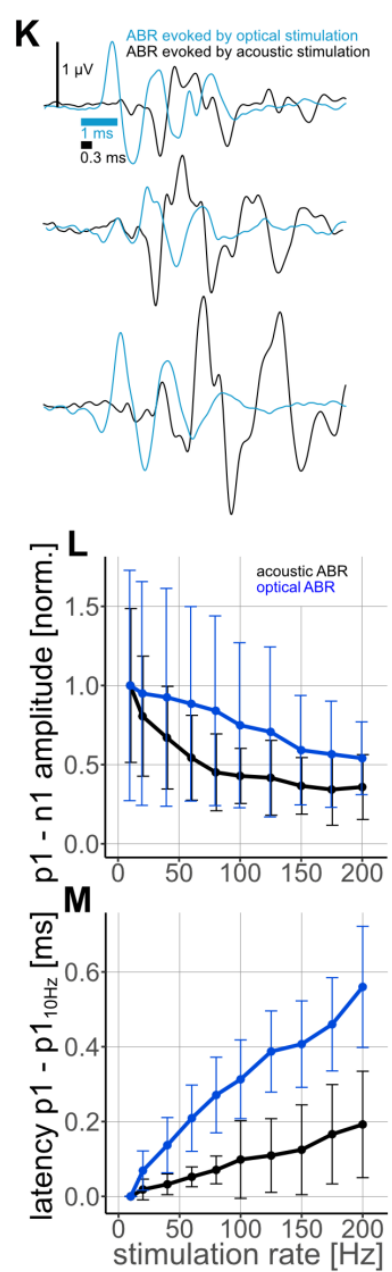

Figure 2. Characterization of optical and acoustic ABR in gerbils. (A) Experimental workflow: $4-12$ weeks after AAVCatCh injection oABRs were recorded and compared to aABRs of the same gerbils as well as to those of non-injected animals. Light of a $473 \mathrm{~nm}$ laser was coupled in by a $200 \mu \mathrm{m}$ optical fiber via a cochleostomy on the middle cochlear turn or through the round window. (B) oABRs from a representative AAV-CatCh-injected adult gerbil with varying light intensities (radiant flux, $1 \mathrm{~ms}$ at $10 \mathrm{~Hz}$ ). Colors code the stimulus parameters. (C) Amplitude of oABR p1 - n1 as a function of light intensity (1 ms light pulses at $10 \mathrm{~Hz}$ ). (D) Latency of oABR p1 as a function of light intensity for the same 20 gerbils (grey) as in C. (E) oABRs from a representative AAV-CatCh-injected adult gerbil with varying stimulus rates (32.2 $\mathrm{mW}$ for $1 \mathrm{~ms}$ ). (F) Amplitude of $\mathrm{OABR} \mathrm{p} 1 \mathrm{-n} 1 \mathrm{as}$ a function of stimulus rate (1 ms light pulses with high light intensities of $21-32 \mathrm{~mW}$ ). (G) Latency of $O A B R$ p1 as a function of stimulus rate for the same 20 gerbils (grey) as in F. Grey lines in $C, D, F, G$ symbolize different animals $(n=20)$, blue line represents mean with vertical error bars indicating SD. Horizontal error bars in C, D represent the SD of the mean light intensity in the respective bin. $(H)$ aABRs (80 dB SPL, $0.3 \mathrm{~ms}$ ) recorded at increasing acoustic click stimulation rate. (I) Quantification of aABR $(n=13) \mathrm{p} 1-\mathrm{n} 1$ amplitude as a function of stimulation rate for acoustic clicks. Grey lines symbolize different animals; black line represents mean with vertical error bars indicating SD. (J) aABR p1 latency plotted against stimulation rate of auditory clicks $(n=13)$. (K) oABRs (blue, $32.2 \mathrm{~mW}, 1 \mathrm{~ms}$ at $10 \mathrm{~Hz}$ ) and aABRs (black, click of $0.3 \mathrm{~ms}, 70 \mathrm{~dB}$, at $10 \mathrm{~Hz}$ ) recorded in three representative AAV-CatCh-injected adult gerbils. (L) p1-n1 amplitude as a function of stimulus rate normalized against p1-n1 amplitude of $10 \mathrm{~Hz}$ aABRs (black) and oABRs (blue); data are shown as mean \pm SD. (M) Latency of p1 as function of stimulation rates normalized against $\mathrm{p} 1$ latency of $10 \mathrm{~Hz}$ aABRs (black) and oABRs (blue) (mean $\pm \mathrm{SD}$ ). Data in C, D, F, $\mathrm{G}, \mathrm{L}$ and $\mathrm{M}$ are pooled for $O A B R$ measurements in acutely and chronically implanted animals. 
Electrophysiological characterization of optical activation of single spiral ganglion neurons: To further validate the CatCh-mediated SGN stimulation and scrutinize the temporal fidelity of stimulation, we performed juxtacellular recordings in vivo from individual SGNs during blue light stimulation from an optical fiber inserted through the round window (18). In order to maximize the chances to record SGNs, we inserted the electrode under visual control directly into the auditory nerve. At $10 \mathrm{~Hz}, 20$ out of 29 SGNs fired one spike to each laser pulse; the remaining 9 SGNs responded with multiple spikes ( $2.6 \pm 0.68$ spikes/pulse), suggesting the presence of 2 separable clusters of SGNs (Fig. 3A, B). The first spike elicited by each pulse in a $10 \mathrm{~Hz}$ train was significantly more synchronized to the stimulus for the SGNs responding with a single spike (jitter $=0.26 \pm 0.49 \mathrm{~ms}$ ) than for the multiple spike-responding SGNs (jitter $=1.66 \pm 1.49 \mathrm{~ms}, p$-value $\leq$ 0.01 , Fig. 3C). Raster plots and peri-stimulus-time-histogram in response to light pulse trains of 10, 100 and $300 \mathrm{~Hz}$ are shown for both a representative single spike and a representative multiple spike unit (Fig. 3D-F and Fig. 3G-I, respectively). Light pulses triggered spikes with high temporal fidelity as evident from high vector strength $(0.85 \pm 0.07)$ up to a stimulus rate of $100 \mathrm{~Hz}$ (Fig. 3J). At higher repetition rates, vector strength dropped to 0 for most SGNs, and remained low but significant in some SGNs (average for all SGNs: vector strength $=0.1 \pm 0.06, p<0.001$, Rayleigh test). The vector strength of non-transduced SGNs in response to acoustic click trains revealed phase-locked responses up to the highest tested rate (500 Hz, Fig. 3J (black dashed line), fig. S6). The highest frequency leading to synchronized responses was $100 \mathrm{~Hz}$ for most of the single spikeresponding SGNs (Fig. 3K). On average the maximal frequency eliciting synchronized responses to optical stimulation was significantly higher for multiple spike-responding SGNs (242.8 \pm 127.2 vs. $121.4 \pm 57.7 \mathrm{~Hz}, p \leq 0.001$, Fig. $3 \mathrm{~L}$ ). In contrast, optogenetically-driven single spike-responding SGNs increased their discharge rate up to $100 \mathrm{~Hz}$, followed by a drop to $\sim 0$ spikes/s at higher rates, whereas multiple spike-responding SGNs sustained the response also for higher rates (Fig. $3 M)$. Above $100 \mathrm{~Hz}$, the discharge rate of multiple spike-responding SGNs to light pulse trains was comparable to acoustic click trains (Fig. 3M, black dashed line). At repetition rates $\geq 100 \mathrm{~Hz}$ single spike-responding SGNs preferentially displayed phasic responses (adaptation ratio $\geq 10, n=12 / 15$ cells) whereas multiple spike-responding SGNs responded in a tonic fashion (adaptation ratio < $10, n=9 / 9$ cells, Fig. $3 N$ ). These recordings directly demonstrate optogenetic SGN excitation and corroborate the ABR results indicating substantial temporal fidelity with optogenetic stimulation. 

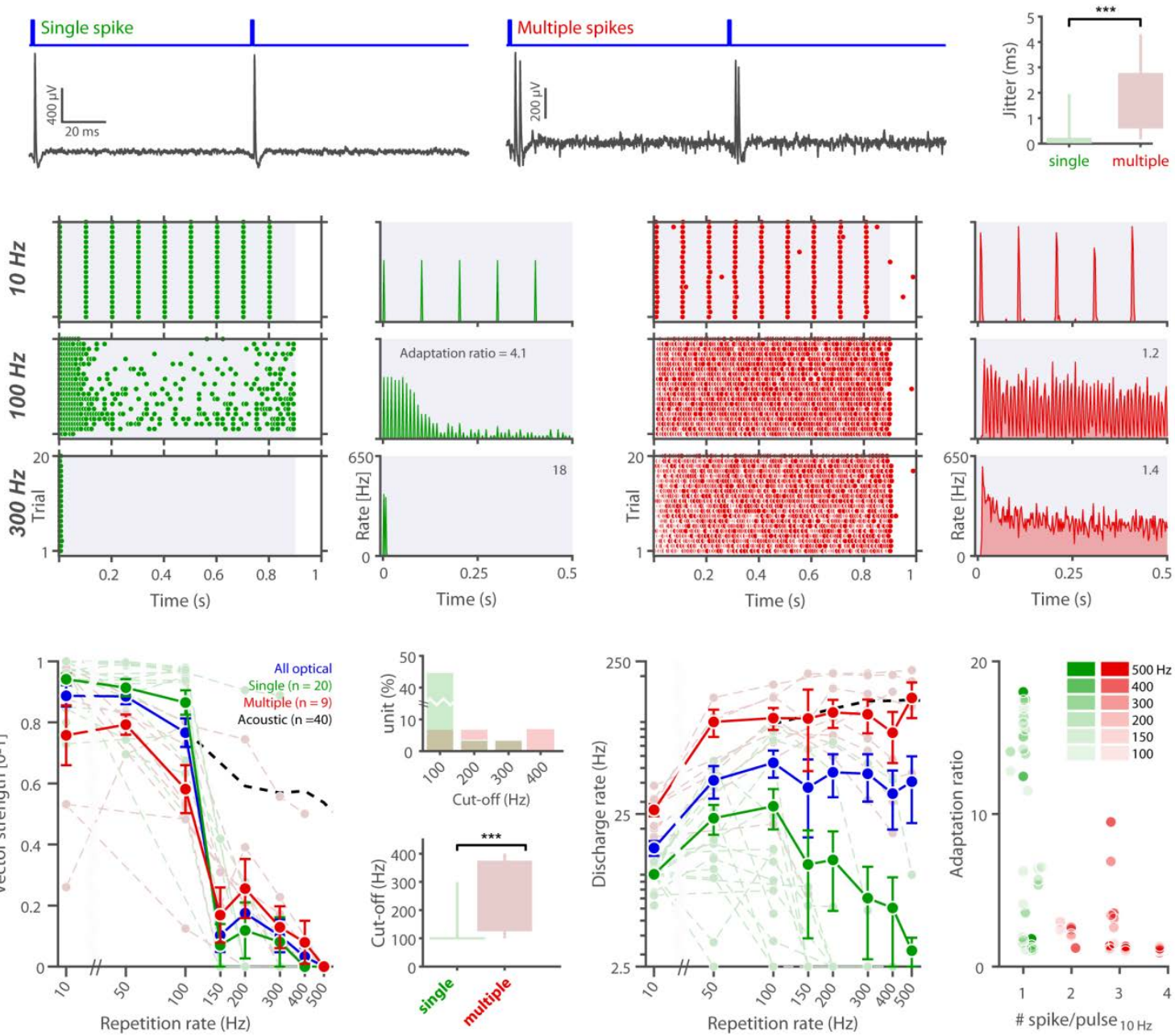

Figure 3: Characterization of SGN responses to optogenetic stimulation. (A-B) Representative juxtacellular SGN recordings during the first $200 \mathrm{~ms}$ of a $900 \mathrm{~ms}$ pulse train $(1 \mathrm{~ms}, 20 \mathrm{~mW})$ with a repetition rate of $10 \mathrm{~Hz}$ of an exemplary single spike- (A) and a multiple spike-responding SGN (B). (C) Quantification of the first spike jitter elicited per light pulse for single spike- (green) and multiple spike- (red) responding SGNs. (D-I) Raster plots and peri-stimulus time histogram of a single spike- (D-F) and a multiple spike-responding SGN (G-I) in response to $900 \mathrm{~ms}$ train pulses of 10, 100 and $300 \mathrm{~Hz}$. (J) Vector strength as a function of repetition rate $(n=29)$. Blue lines correspond to the population mean, green lines to single spike-responding SGNs, red lines to multiple spike-responding SGNs and dashed black line to acoustic stimulation of control SGN (see figure S4 for details). (K, L) Distribution (K) and quantification (L) of the cut-off frequency (highest frequency with a significant vector strength) for single spike- (green) and multiple spike- (red) responding SGNs. (M) Discharge rate as a function of repetition rate $(n=29)$. (N) Adaptation ratio as a function of the number of spikes per light pulse in response to $10 \mathrm{~Hz}$ light pulse train. A color scale is used to represent the repetition rate. Data are expressed as mean \pm SEM, and the statistical difference between groups were tested by Mann-Whitney $\mathrm{U}$ test $(* * * p$-value $\leq 0.001)$

Chronic oCl development and electrophysiological characterization: In order to study a possible percept elicited by optogenetic SGN-stimulation we developed and implanted a chronic fiberbased single-channel oCl in gerbils 4-8 weeks after AAV-CatCh or phosphate buffered saline (PBS control) injection (Fig. 4A). Functionality of the $\mathrm{OCl}$ was monitored by regular oABR recordings (every other day during the first week after surgery and once a week afterwards), eliciting stable 
responses in AAV-CatCh injected, but not PBS-injected animals (AAV-CatCh: $n=7$, PBS: $n=5$ [ 2 of these 5 animals have been implanted and measured chronically], Fig. 4B). Chronic oCl produced stable and reliable responses over more than 3 weeks in all gerbils. Two animals were continuously tested until after behavioral experiments and displayed stable responses for more than 100 days (Fig. 4C). oABR amplitudes were similar between the last day of behavioral testing and the day after surgery $(1.33 \pm 0.86 \mu \mathrm{V}$ after surgery, $0.99 \pm 0.42 \mu \mathrm{V}$ after behavioral testing, $p=0.14, n=7$ ) indicating a largely stable physiological response over time (fig. S7A). The mean oABR threshold $24 \mathrm{~h}$ after chronic oCl surgery amounted to $6.9 \pm 2.46 \mathrm{~mW}$ and was significantly elevated compared to oABRs measured in acute experiments $(4.6 \pm 2.8 \mathrm{~mW}, \mathrm{n}=7$ for chronic, $\mathrm{n}=14$ for acute
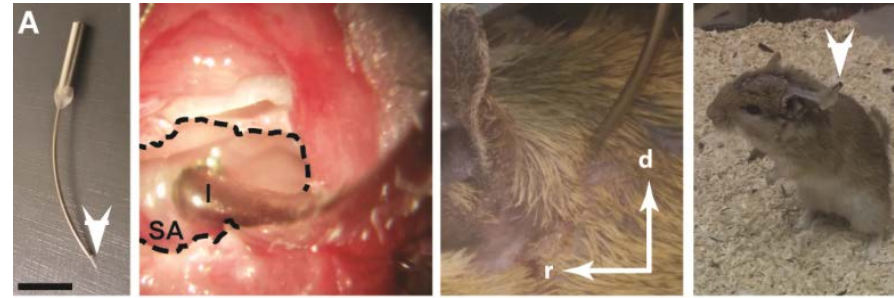

B CatCh - injected

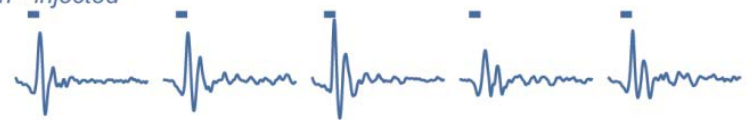
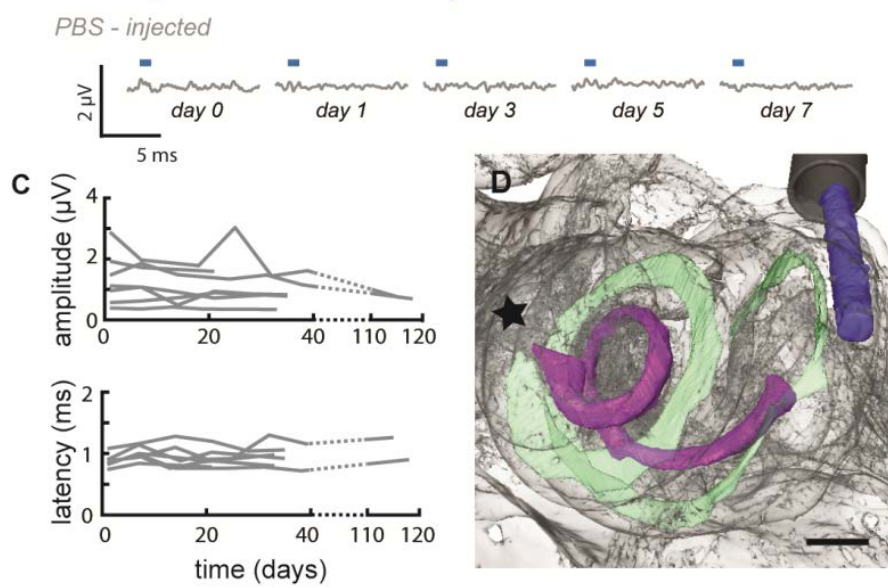

Figure 4. Chronic fiber-based, single-channel oCl. (A) Left: Chronic fiber-based single-channel oCl (arrow) housed in a stainless-steel capillary (scale $1 \mathrm{~cm}$ ), center left: $\mathrm{oCl}(\mathrm{I})$ placed through the round window into the scala tympani just above the stapedial artery (SA); dashed line: bullostomy; center right: oCl 3 weeks after surgery ( $r$ : rostral, d: dorsal). Right: Gerbil, 3 days after implantation. Arrowhead: ferrule for optical fiber connection (B) oABRs of a AAV-CatCh injected animal (top) at the day of implantation (day 0) as well as 1, 3, 5 and 7 days after implantation, no oABR was found in PBS-injected animals (bottom), blue bars indicate optical stimuli. (C) oABR amplitudes (p1 $n 1$, top) and latencies ( $p 1$, bottom) during the course of behavioral experiments ( $\mathrm{n}=7$ up to day 40 post implantation; two animals to day 115); each line correspond to a single animal. (D) X-ray tomography of the cochlea including implant to confirm the fiber's position after behavioral experiments. Green: Basilar membrane. Purple: Rosenthal's canal. Black: Steel capillary. Blue: Optical fiber. Star: Cochlear apex. Scale bar: $500 \mu \mathrm{m}$.

measurements, $p=0.038$, fig. S7B, C). However, thresholds decreased over time and 3 weeks after implantation the responses were comparable to acute experiments $(4.7 \pm 2 \mathrm{~mW}, \mathrm{p}=0.44$, fig. S7C). The position of the implanted fiber relative to the spiral ganglion was investigated with X-ray tomography of the cochlea (19). In these studies on five postmortem specimens the aperture always pointed slightly off the central axis of the modiolus (Fig. 4D, fig. S8). Therefore the optical fiber was not optimally orientated toward the SGNs. 
Behavioral assessment of chronic oCl: In order to test whether the electrophysiological responses triggered by optical stimulation had functional effects on behavior, we chose the shuttle-box paradigm of negative reinforcement learning (Fig. 5A-C). The test has been successfully used for auditory studies in gerbils and employs mild electrodermal stimulation of the paws to reinforce crossing a barrier to a safe compartment upon a sensory target stimulus (20). To adapt the animals to the arena without risk of damage to the oCl we performed one session with acoustic click trains (Fig. 3D). This session was followed by subsequent optogenetic training. Gerbils learned to react to optogenetic stimuli over roughly a week, typically reaching more than $80 \%$ hitrate on day 6 of the daily training program (Fig. 5E).
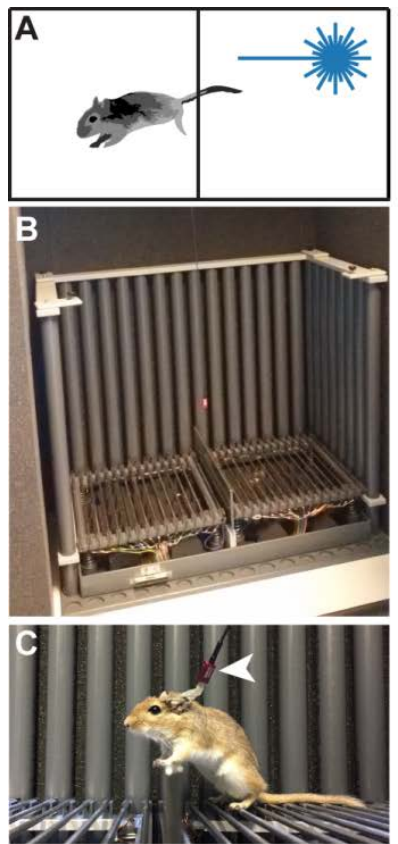

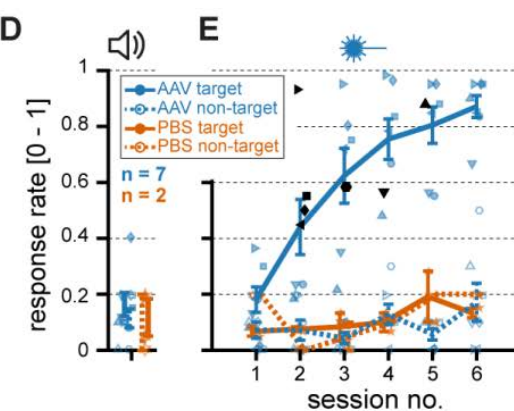

I

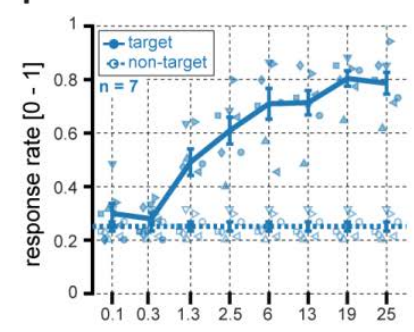

light intensity $[\mathrm{mW}]$

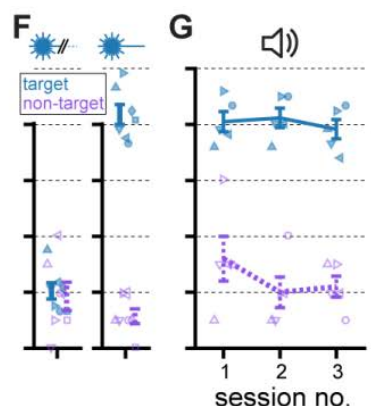

$J$

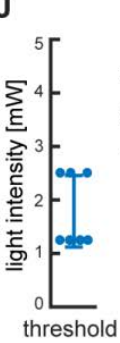

K

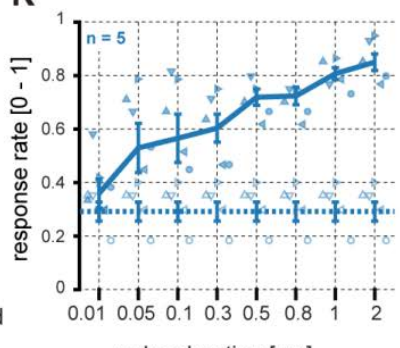

pulse duration $[\mathrm{ms}]$

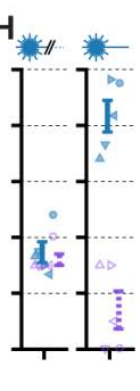

$\mathbf{L}$

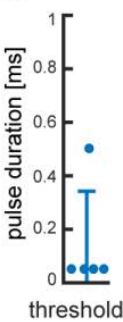

Figure 5. Optogenetically cued avoidance behavior. (A) Outline of the task: After an initial training period, the gerbil changes the compartments of the shuttle-box upon stimulus presentation (acoustic or optogenetic). (B-C) Picture of the shuttle-box (front wall removed) in a sound-attenuating chamber (B) and of a gerbil in the setup (C). Arrowhead: interface between ferrule and optical fiber. (D-E) Behavioral performance during the course of shuttle-box training for a single habituation session using acoustic stimuli (D) and the subsequent training period using optogenetic stimulation (E). Solid and dashed lines show mean hit and sham rates \pm SEM for AAV-CatCh- (blue, $n=7$ ) and PBS-injected (orange, $\mathrm{n}=2$ ) animals. Filled and empty markers show hit and sham rates, respectively, for each individual animal.(F): Hit rates in response to target (blue) and non-target (purple) trials during a control experiment with a blocked (left) and reopened (right) beam path. (G): Transfer from optogenetic to acoustic cues for avoidance behavior. (H) Second control, performed after transfer to acoustic stimulation. Different marker shapes correspond to different animals and are consistent throughout the figure. (I-L) Average hit rates and behavioral thresholds of individual animals for light power $(I, J)$ and pulse duration $(K, L)$. Solid and dashed lines indicate mean hit and sham rates \pm SEM across all animals (I: $n=7$, $K: n=5)$. Filled and empty markers show hit-rates for target and non-target trials, respectively. Behavioral thresholds were defined as the weakest stimuli eliciting significant performance in each individual animal (Chi-Square-Test, $p<$ 0.01). 
Spontaneous crossing during non-target trials (interspersed trials without cue stimuli and negative reinforcement) occurred in typically $20 \%$ of these trials. Our results showed that learning strictly required opsin-expression by SGNs: hit-rates for target and non-target trials did not differ in PBSinjected animals, even though they were stimulated with relatively strong light pulses (10 ms $\times 30$ $\mathrm{mW}=300 \mu \mathrm{J})$. Hit-rates of AAV-CatCh-injected animals broke down upon blocking the light path in the ferrule (still producing light emission at the coupler but not in the cochlea itself) and were restored immediately after re-opening the path, suggesting that the behavioral responses were not induced by visual cuing (the blue-light emission at the coupler) or by other sensory cues (Fig. 5F). Optogenetically-trained gerbils transferred the avoidance behavior to acoustic stimulation: hit-rates in acoustic stimulation sessions were comparable to hit rates using optogenetic stimulation from the first acoustic session on and two out of five tested gerbils crossed the barrier to the safe compartment already in response to the first acoustic stimulus, suggesting at least some degree of generalization between the percepts elicited by optogenetic and acoustic stimulation (Fig. 5G).

Following basic shuttle-box training, we estimated behavioral thresholds for light intensity and duration of the laser pulses. Here, within one session, target trials of either different light intensities or different pulse durations, as well as non-target trials, were presented pseudorandomly. After six identical sessions (60 repetitions of each trial type), for each light intensity and pulse duration, we compared the hit-rate of individual animals for each stimulus condition to the hit rate of non-target trials in the same animal $(p<0.01)$. Testing different light intensities ranging from $0.1-25 \mathrm{~mW}$, we found that the hit-rate dropped sharply below $1.8 \mathrm{~mW}$ on average $(1.8 \mu \mathrm{J}$ per pulse, Fig. 5I, J). This is below the thresholds obtained for acute recordings of oABR (4.6 $\mu \mathrm{J}$, see above) and single neuron responses in auditory cortex (3.11 $\mu \mathrm{J}$, see below). The average threshold for stimulus duration was approximately $140 \mu$ s (individual thresholds for 4 out of 5 animals were as short as $50 \mu \mathrm{s}$; Fig. $5 \mathrm{~K}, \mathrm{~L}$ ).

Neuronal response properties in primary auditory cortex and cochlear spread of excitation: In an effort to further characterize the auditory signaling elicited by $\mathrm{oCl}$, we performed in vivo extracellular recordings from layer IV of the contralateral primary auditory cortex (AI) while stimulating the AAV-CatCh-transduced SGNs at the base of the cochlea with blue light (Fig. 6A). When an Al neuron was isolated, we first mapped its best frequency (BF, sound frequency for which the maximal spike rate was obtained). BFs progressed to higher frequencies along electrode tracks and covered the tonotopic range (Fig. 6B). A total of 43 neurons in Al were 
studied of which 38 responded to pure tones with frequencies in the hearing range of gerbils. Out of these 20 neurons responded to optogenetic SGN stimulation (1 ms pulse length, approximately $1 \mathrm{~Hz}$ repetition rate) albeit at different thresholds. Among the total set of 43 neurons studied, 22 responded to individual light pulses.

A range of response types was found: the majority of the neurons showed monotonically increasing firing rates with growing light intensity, whereas the firing rate of other neurons first rose but then decreased upon further enhancing light intensity (non-monotonic response, Monotonicity index $<0.5$; Fig. 6C, D). Neurons with high BF $(8-32 \mathrm{kHz})$ had a mean threshold of 1.4 $\pm 1.0 \mathrm{~mW}(\mathrm{SD}, \mathrm{n}=5)$ consistent with the oABR and behavioral thresholds. Thresholds of lower BF neurons $(<8 \mathrm{kHz})$ were significantly higher $(8.0 \pm 9.5 \mathrm{~mW}, \mathrm{n}=12, \mathrm{p}=0.046)$, indicating that limited light spread in the cochlea occurs (Fig. 6E, fig. S9).

We followed up the aspect of light spread within the cochlea by a Monte Carlo ray tracing model based on an x-ray imaged gerbil cochlea with the $200 \mu \mathrm{m}$ optical fiber implanted into scala tympani via the round window (fig. S10A). We modeled three million rays $(\lambda=473 \mathrm{~nm})$ in order to investigate the spread of excitation at the center of Rosenthal's canal, where the somata of SGNs are housed. With a fiber output of $1 \mathrm{~mW}$ (chosen from the thresholds for most sensitive neurons recorded in $\mathrm{Al}$ in the range around $10 \mathrm{kHz}$ ) we estimated the threshold for neuronal excitation in Rosenthal's canal to be $0.06 \mathrm{~mW} / \mathrm{mm}^{2}$ and the bandwidth of excitation to range from $10-14.7$ $\mathrm{kHz}$ (0.56 octaves, fig. S10B, C). As expected, we observed an increased light spread with fiber outputs of 5 and $10 \mathrm{~mW}$ that reached super-threshold irradiances at cochlear regions with BFs of $5.7-26.3 \mathrm{kHz}$ (2.2 octaves) and $3.05-32.1 \mathrm{kHz}$ (3.4 octaves), respectively (fig. S9B, C). For these stronger light intensities, additional peaks of excited SGNs were observed: $0.51-0.87(5 \mathrm{~mW})$ and $0.39-1.34 \mathrm{kHz}(10 \mathrm{~mW}$, fig. S1OB, C). We note that the projection of light from the round window does not represent the appropriate optical stimulation strategy for a future oCl.

Therefore, we also modelled the spread of excitation for light delivered by a small optical fiber (10 $\mu \mathrm{m}$ of diameter with a $0.1 \mathrm{NA}$ ) as an ideal light emitter placed at four different locations in the center of scala tympani along the tonotopic axis each facing Rosenthal's canal that houses the SGN somata (fig. S10D). Our simulations revealed narrow light spread and indicated that excitation of distinct populations of SGNs can be achieved using optogenetic stimulation at emitter intensities in the range of microwatts (fig. S10E, F). 

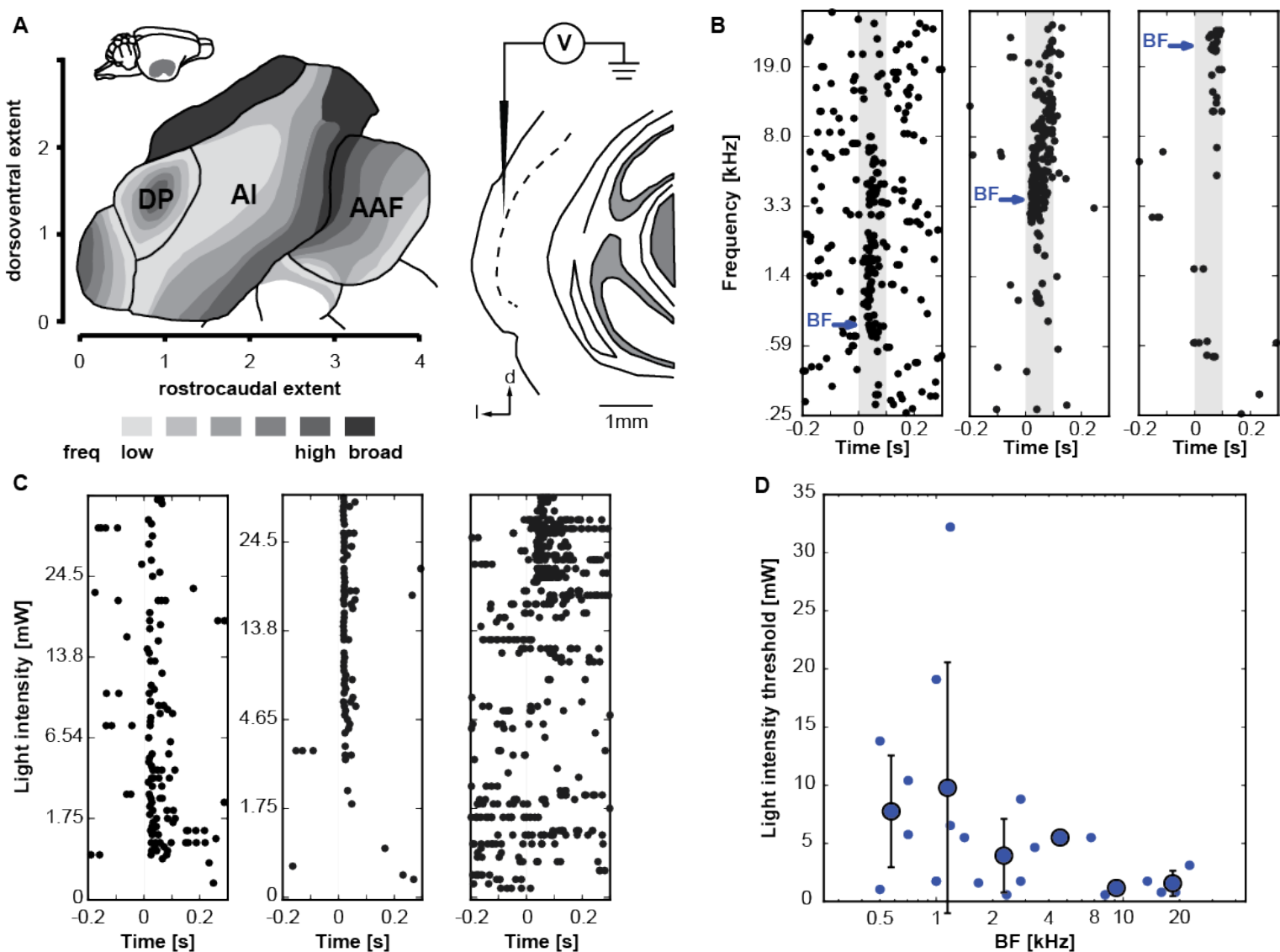

Figure 6. Responses of single Al neurons to optogenetic SGN stimulation. (A) Schematic shows the experimental approach: after initial mapping of the tonotopic representation to identify field Al of the contralateral auditory cortex, a single tungsten electrode was tangentially inserted and advanced to study the activity of single Al neurons (single unit) within the thalamo-recipient layer IV. (B) Neuronal responses of 3 individual neurons to pure tones along a representative recording track. The best frequencies $(\mathrm{BF})$ of neurons varied systematically along each electrode track where BFs were lower dorsally and increased ventrally. (C) Neuronal responses to individual laser pulses $(1 \mathrm{~ms}$, at 1 $\mathrm{Hz}$ ) applied to the contralateral cochlea in a monotonic (left) and non-monotonic unit (right). (D) Distribution of monotonicity-indices of all recorded unit. Dashed bar indicates a monotonicity index of 0.5 to discriminate between monotonic and non-monotonic units. (E) Comparison of acoustic and optical response properties. BFs are plotted against the corresponding laser threshold for each single unit $(n=20)$.

Hearing restoration in a gerbil model of ototoxic deafness: Finally, we explored the potential of chronic oCl for restoring hearing in a gerbil model of ototoxic deafness. After measuring aABRs, AAV-CatCh injected gerbils were trained to detect $70 \mathrm{~dB}$ click trains using the shuttle-box paradigm. Animals were then deafened by bilateral, intracochlear injections of kanamycin $(2 \mu \mathrm{l}$ of $100 \mathrm{mg} / \mathrm{ml},(21)$ ). Deafness was confirmed by absence of aABR (up to the highest sound pressure level amenable to our sound system (110 dB SPL) and the inability to perform shuttle-box behavior with acoustic stimulation (Fig. 7A).

We then implanted single-channel oCls and demonstrated optogenetic activation of the auditory pathway by means of OABR (Fig. 7B, C). Post-hoc immunofluorescence analysis indicated the 
absence of hair cells from kanamycin-deafened ears (Fig. 7D). OABR thresholds of kanamycintreated chronically implanted gerbils were comparable to kanamycin-untreated chronically implanted gerbils (mean \pm SD: $7.9 \pm 3.5 \mathrm{~mW}, \mathrm{n}=4$ in treated animals; $6.9 \pm 2.4 \mathrm{~mW}, \mathrm{n}=7$ in untreated animals). Next, we tested whether these acoustically trained animals, which could not use acoustic cueing any longer upon deafening, could transfer the learned behavior or would (re)learn the behavior by the optogenetic stimulation (Fig. 7E-G). One of the four gerbils tested immediately crossed to the safe compartment upon the first optogenetic stimulation with a hitrate of more than $80 \%$, suggesting transfer of the acoustically cued behavior to the optogenetic cue; the other animals learned to use the optogenetic cue within a few days. On average, acoustically pre-trained and kanamycin-treated oCl implanted gerbils took 2.0 days $( \pm 0.8 \mathrm{SD}, \mathrm{n}=$ 4) to re-learn the task using an optical cue compared to $3.5 \pm 1.7$ days ( $n=4$, not different) when learning the task using acoustic stimulation in naive animals. As expected, the deafened gerbils could no longer use the acoustic cue even at the highest sound pressure levels amenable to our shuttle-box system (100 dB SPL, Fig. 7H). In conclusion, oCl stimulation of SGNs restored activation of the auditory pathway in a gerbil model of human ototoxic deafness as revealed by analysis of physiological and behavioral responses. 

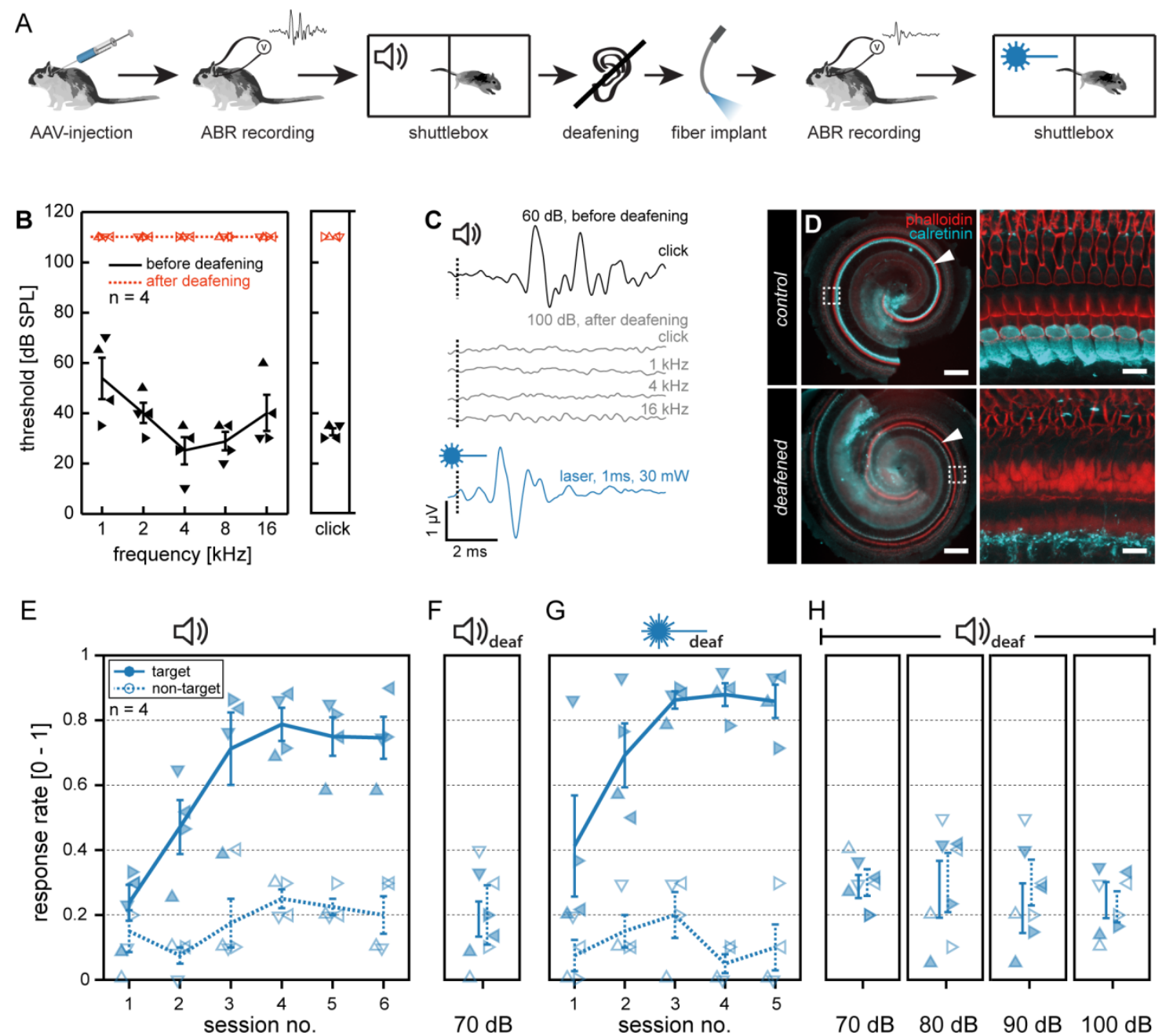

Figure 7. Restoration of hearing in a gerbil model of ototoxic deafness (A) Experimental workflow: after AAV-CatChinjection auditory brainstem responses were recorded, gerbils were trained in the shuttle-box for six days using acoustic stimulation and finally deafened by bilateral intracochlear injections of Kanamycin solution. After deafness was confirmed using aABRs and a single shuttle-box session, animals were implanted with an optical fiber. Subsequently, OABRs were recorded and shuttle-box experiments were performed using optogenetic stimulation. (B) (B) aABR thresholds (individual data and mean $\pm S E M, n=4$ ) for logarithmically spaced pure tones ranging from 1 to 16 $\mathrm{kHz}$ as well as click stimuli, before (black triangles and solid line) and after deafening (red triangles and dashed line). (C) Representative $\mathrm{aABR}$ in response to a $60 \mathrm{~dB}$ click stimulus before deafening (black, top.). Absence of aABRs in response to $100 \mathrm{~dB}$ click stimuli as well as 1,4 and $16 \mathrm{kHz}$ pure tones after deafening (grey, middle). oABRs in AAV-CatCh injected oCl-implanted deafened animal (blue, bottom). Vertical dashed lines indicate stimulus onsets. All traces were recorded from the same animal. (D) Histological verification of IHC loss upon deafening. Overview of the apical turn of the organ of Corti in a control animal (top left) and a kanamycin deafened animal (bottom left). Phalloidin was used to stain actin prominently expressed in hair cells and supporting cells (red), Calretinin was used to stain spiral ganglion neurons and IHCS (cyan): arrowheads point to the location of IHCs. Note the lack of IHCs in the deafened animals. Scale bar: $100 \mu \mathrm{m}$. Right: Magnification of the regions outlined on the left side, respectively. Note that spiral ganglion afferents are still present in the deafened animal (bottom right). Scale bar: $10 \mu \mathrm{m}$. (E) Learning curves of shuttle-box behavior using $70 \mathrm{~dB}$ acoustic stimulation before deafening $(E), 70 \mathrm{~dB}$ after deafening (F), optogenetic cues $(\mathrm{G})$ and acoustic cues up to $100 \mathrm{~dB}$ after deafening $(\mathrm{H})$. Solid and dashed lines indicate the mean hit-rates \pm SEM for target and non-target trials, respectively. Filled and empty markers indicate the individual rates for each animal $(n=4)$. Different marker shapes correspond to different animals and are consistent throughout the figure. 


\section{Discussion}

Here, we established the Mongolian gerbil as a rodent model for developing the oCl. We achieved AAV-mediated expression of CatCh in cochlear SGNs of adult Mongolian gerbils and characterized optogenetic stimulation by electrophysiology of single neurons and neural populations, as well as by behavioral analysis. CatCh-mediated optogenetic SGN stimulation drove the auditory pathway up to the primary auditory cortex and elicited avoidance behavior. Intramodiolar AAV-CatCh injection achieved CatCh expression in approximately 50\% of the injected cochleae, where about $30 \%$ of the SGNs were transduced. We did not find evidence for viral spread beyond the injected ear. All three cochlear turns were transduced to a similar extent which contrasts the primarily basal expression obtained with transuterine AAV-injection (12). The injection caused loss of SGNs (25\%) that is likely to be due to the pressure injection, as it was also found in AAV-CatCh injected ears where no opsin expression was observed. As calretinin is thought to stain a subset but not all SGNs, the use of calretinin staining for identification of SGNs might have caused an underestimation of the number of SGNs and an overestimation of the transduction rate (22). Hence, despite calretinin labelling the vast majority of SGNs in our experience, future studies should employ more global neuronal markers to indiscriminately label all SGNs, such as beta III tubulin (23). Nonetheless, despite the relatively low SGN transduction rate in the present study, we obtained electrophysiological and behavioral responses in adult CatCh-positive gerbils. The finding that $O A B R$ could already be elicited when $10 \%$ of the SGNs were transduced contrasts our previous study in which at least $40 \%$ of the transduced SGNs were needed in order to support optophysiological responses (12).

In contrast to the very large (10s - 100s of $\mu \mathrm{V})$ and delayed ( $3 \mathrm{~ms}$ ) optically-evoked far-field potentials reported for transgenic mice and rats, we observed smaller and earlier potentials with targeted viral optogenetic manipulation of the ear of gerbils (12). These differences might be due to a more localized expression of CatCh in SGNs using injections into the adult spiral ganglion, compared to the Thy1.2-driven broad neuronal expression of ChR2 employed in our previous work that might have resulted in optogenetic stimulation extending to structures beyond the auditory pathway. In support of this hypothesis, in the present study oABRs and aABRs were more similar in amplitude and showed the expected short latency for direct optogenetic SGN stimulation. OABRs remained sizable for stimulus rates of up to $200 \mathrm{~Hz}$, and the OABR-amplitude reduction of 1.5 fold observed when raising stimulus rate from 10 to $150 \mathrm{~Hz}$, was favorable compared to the $\mathrm{AABR}$ amplitude reduction $(2.5-\mathrm{fold})$, indicating that temporal fidelity of 
optogenetic coding might approach near physiological performance. We consider this an important advance over our previous work, which indicated much lower temporal fidelity (responses were only found up to $70 \mathrm{~Hz}$ ) (12). Our juxtacellular recordings from single SGNs confirmed specific, synchronized optogenetic excitation of SGNs up to repetition rate of $100 \mathrm{~Hz}$ for all recorded fibers. This result supports the temporal fidelity reported by $O A B R$ and is in accordance with the closing kinetic of CatCh (16 ms measured at room temperature) (14). The lower spike time fidelity and limited spike probability at stimulus rates beyond $100 \mathrm{~Hz}$ observed are likely compensated at the neuronal population level, as several SGNs jointly encode information from each place of the tonotopic map (24).

We found considerable differences in OABR amplitudes among the gerbils. This variability might be due to differences in transduction rate, amount of CatCh expression among the transduced cells and positioning of the optical fiber. Nonetheless, the key features such as the dependence of p1-n1 amplitude and p1-latency on the radiant flux and rate of stimulation were comparable among different CatCh transduced animals. We could not determine the exact light propagation from the fiber aperture within the cochlea and hence, it was not possible to determine the precise irradiance at a given point of the SGNs. However, we employed a Monte Carlo ray tracing model to estimate the irradiance at the site of neural excitation. Considering the maximum light intensity used in our behavioral experiments $(30 \mathrm{~mW})$ we estimate the maximal irradiance at the site of SGN somata to be approximately $2 \mathrm{~mW} / \mathrm{mm}^{2}$, which is within the safe range for optogenetic in vivo applications (up to $\sim 75 \mathrm{~mW} / \mathrm{mm}^{2}$ for $0.5-50$ ms pulses; even up to $\sim 300 \mathrm{~mW} / \mathrm{mm}^{2}$ in some studies) $(25,26)$. Furthermore, the oABR long-term stability for $>100$ days observed here suggests absence of tissue damage due to optogenetic stimulation.

In $40 \%(8 / 20)$ of the tested animals OABR increased in amplitude when increasing light intensity over more than one order of magnitude, indicating an output dynamic range of optical coding > $20 \mathrm{~dB}$. As the response did not plateau in the majority of animals the average apparent dynamic range of $16 \mathrm{~dB}$ is likely an underestimation. This contrasts with coding with eCl where typically < $10 \mathrm{~dB}$ dynamic range were reported (4). This might reflect the lower spread of excitation with optical stimulation as well as differences in the expression of CatCh among SGNs at the tonotopic place of stimulation. Recordings of oABR revealed a minimal stimulus duration of $<200 \mu \mathrm{s}$ and an energy threshold of approximately $4.6 \mu \mathrm{J}$ per pulse. Recordings of single neuron firing in primary auditory cortex confirmed this energy threshold. A recent study in awake primates demonstrated 
that approximately $40 \%$ of neurons in the auditory cortex could be driven by contralateral eCl stimulation (27) while we observed $52 \%$ of neurons driven by oCl in the gerbil.

Our chronic experiments employed a fiber-based single-channel oCl that enabled stable stimulation over several weeks. This allowed longitudinal tracking of oABR and behavioral analysis of oCl function in individual gerbils. The absence of learning by PBS injected animals rules out the sensation of opto-thermal or opto-acoustic effects in the cochlea as a behavioral cue. In AAVCatCh injected gerbils, behavioral estimates of thresholds for light pulse intensity and duration were lower than those obtained in our OABR experiments, indicating that activation of few neurons might suffice to trigger SGN stimulation. Still, behavioral experiments might have overestimated the energy threshold of $\mathrm{oCl}$, because post-mortem X-ray phase-contrast tomography of the implanted cochlea indicated non-optimal projection to the spiral ganglion from the fiber. Therefore, the behavioral estimate of the energy threshold $(2 \mu \mathrm{J})$ is lower than the threshold obtained with physiological methods but might still overestimate the light required for triggering auditory perception.

Nonetheless, lowering the energy requirement, which currently exceeds the energy per pulse in $\mathrm{eCl}(0.2 \mu \mathrm{J}$ for biphasic pulses of $80 \mu \mathrm{s}$, remains an important objective in order to fully capitalize on the potential of $\mathrm{oCl}$ for miniaturizing the stimulation site (for example using $50 \mu \mathrm{m}$ sized LEDs compared to hundreds of $\mu \mathrm{m}$ for $\mathrm{eCl}$ ), avoid potential phototoxicity and thermal effects as well as to enable acceptable battery lifetimes in future clinical oCl $(4,28,29)$. Such efforts include achieving greater transduction rates as well as stronger expression by optimizing virus-capsid, promoter and virus preparation and better positioning of emitter(s) in scala tympani by arrays of $\mu L E D s$ or waveguides (29). Indeed, our optical modelling indicated that placing an "ideal emitter" into scala tympani might dramatically lower the energy required. Moreover, we expect that the higher energy requirements of the oCl due to the larger number of stimulation channels compared to eCl can be offset by lower rates of stimulation closer to the physiological SGN firing rates (24).

Studies of acoustically- and optogenetically-guided avoidance behavior in the shuttle-box indicated that acoustic percepts were formed with both modes of stimulation of the auditory pathway (sound and light). Interestingly, immediate transfer of optogenetically-trained behavior to acoustical stimulation was observed in all five gerbils investigated whereas the opposite (transfer of acoustically-induced behavior to optogenetic stimulation) was found for one of four 
acoustically-trained, subsequently deafened and oCl-implanted gerbils. By combining bilateral deafening by intracochlear kanamycin injection with chronic oCl, our results suggest that restoration of auditory function might be achieved with oCl.

Taken together, the results of our present study demonstrate the feasibility of optogenetic activation of the auditory system following AAV-CatCh transduction in SGNs in adult gerbils. We find good temporal precision of optogenetically driven spiking in CatCh-expressing SGNs. Activity propagates up to primary auditory cortex. Furthermore, acoustic perception was confirmed in behavioral experiments with hearing and deaf animals. Our work increases the viability of cochlear optogenetics as an alternative hearing restoration technology and lays the foundation for in depth preclinical research.

Next to the far from complete viral transduction of the spiral ganglion and the employment of CatCh, which has a relatively slow deactivation, our study was limited by the use of oCls based on single optical fibers with non-ideal light projection onto SGNs. Our ray tracing model supports the expected potential for more spatially confined stimulation of SGNs when choosing appropriate positioning of emitters. Next steps of developing oCls towards clinical application should include studies of spectral resolution combining appropriate multichannel stimulation like waveguide array-based or $\mu$ LED-based multi-channel oCls with electrophysiological and/or psychophysical studies of frequency and/or emitter discrimination (29). Such investigation of the cochlear spread of excitation with more realistic oCls will help to determine the number of independently usable stimulation channels of future oCls. 


\section{Materials and Methods}

Study design: We aimed to investigate optogenetic viral constructs to transduce spiral ganglion neurons in an adult rodent model for their potential for optogenetic hearing restoration. Animals transduced with a suitable construct were further investigated with physiological, immunohistochemical, imaging and behavioral methods.

Statistical Analysis: All data displayed in the figures is expressed as mean \pm standard deviation, unless stated otherwise. Unless noted otherwise an alpha level of 0.05 was considered significant.

SGN recordings: The presence of two subpopulations of spiral ganglion neurons was confirmed using Akaike's information criterion computed on the number of spike per pulse in response to 10 $\mathrm{Hz}$ light pulse train. Significance of different cutoff-frequencies in these subpopulations was confirmed using the Mann-Whitney U test. Vector strength of SGNs was checked for significance using the Rayleigh-Criterion. If the Rayleigh statistic L was larger than 13.8, the null hypothesis was rejected at the 0.001 significance level and insignificant vector strengths were set to 0 .

oABRs: An independent two-sample t-test was used to analyze potential threshold differences of optically evoked ABRs in acute measurements vs chronically implanted animals. The relation between OABR amplitude and YFP-expressing SGNs was analyzed by using simple linear regression.

Behavior: As the behavioral output of an animal in the shuttlebox (compartment change/no compartment change) is a categorical one, sham and hit rates for each animal were compared against each other using a $\mathrm{Chi}^{2}$-test $(\mathrm{p}<0.01)$ to determine significant performance.

Auditory cortex recordings: Threshold differences for optical stimulation of auditory cortex units tuned to low $(1-8 \mathrm{kHz})$ and high $(8-32 \mathrm{kHz})$ frequencies were analyzed using a one-sided independent two-sample t-test. Statistical analyses were performed using Matlab R2016a, R 3.4.4 and Python 3.6. 


\section{References and Notes:}

1. WHO, Primary Ear \& Hearing Care Training Resource (World Health Organization, 2006).

2. B. S. Wilson, M. F. Dorman, Cochlear implants: current designs and future possibilities. J. Rehabil. Res. Dev. 45, 695-730 (2008).

3. J. C. Middlebrooks, J. A. Bierer, R. L. Snyder, Cochlear implants: the view from the brain. Curr. Opin. Neurobiol. 15, 488-493 (2005).

4. F.-G. Zeng, S. Rebscher, W. V. Harrison, X. Sun, H. Feng, Cochlear Implants:System Design, Integration and Evaluation. IEEE Rev. Biomed. Eng. 1, 115-142 (2008).

5. A. Kral, R. Hartmann, D. Mortazavi, R. Klinke, Spatial resolution of cochlear implants: the electrical field and excitation of auditory afferents. Hear. Res. 121, 11-28 (1998).

6. J. C. Middlebrooks, R. L. Snyder, Auditory prosthesis with a penetrating nerve array. J. Assoc. Res. Otolaryngol. JARO. 8, 258-279 (2007).

7. R. V. Shannon, Multichannel electrical stimulation of the auditory nerve in man. II. Channel interaction. Hear. Res. 12, 1-16 (1983).

8. L. M. Friesen, R. V. Shannon, D. Baskent, X. Wang, Speech recognition in noise as a function of the number of spectral channels: Comparison of acoustic hearing and cochlear implants. J. Acoust. Soc. Am. 110, 1150 (2001).

9. G. S. Donaldson, H. A. Kreft, L. Litvak, Place-pitch discrimination of single- versus dualelectrode stimuli by cochlear implant users (L). J. Acoust. Soc. Am. 118, 623-626 (2005).

10. J. L. Pinyon et al., Close-field electroporation gene delivery using the cochlear implant electrode array enhances the bionic ear. Sci. Transl. Med. 6, 233ra54-233ra54 (2014).

11. C.-P. Richter et al., Spread of cochlear excitation during stimulation with pulsed infrared radiation: inferior colliculus measurements. J. Neural Eng. 8, 056006 (2011).

12. V. H. Hernandez et al., Optogenetic stimulation of the auditory pathway. J. Clin. Invest. 124, 1114-1129 (2014).

13. T. Mager, P. G. Wood, E. Bamberg, Optogenetic Control of Ca2+ and Voltage-Dependent Large Conductance (BK) Potassium Channels. J. Mol. Biol. 429, 911-921 (2017).

14. S. Kleinlogel et al., Ultra light-sensitive and fast neuronal activation with the $\mathrm{Ca}^{2}+-$ permeable channelrhodopsin CatCh. Nat. Neurosci. 14, 513-518 (2011).

15. N. C. Klapoetke et al., Independent optical excitation of distinct neural populations. Nat. Methods. 11, 338-346 (2014).

16. A. E. Hight et al., Superior temporal resolution of Chronos versus channelrhodopsin-2 in an optogenetic model of the auditory brainstem implant. Hear. Res. 322, 235-241 (2015). 
17. W. Chen et al., Restoration of auditory evoked responses by human ES-cell-derived otic progenitors. Nature. 490, 278-282 (2012).

18. A. Huet et al., Sound coding in the auditory nerve of gerbils. Hear. Res. (2016), doi:10.1016/j.heares.2016.05.006.

19. M. Bartels, V. H. Hernandez, M. Krenkel, T. Moser, T. Salditt, Phase contrast tomography of the mouse cochlea at microfocus x-ray sources. Appl. Phys. Lett. 103, 083703 (2013).

20. H. Schulze, H. Scheich, Discrimination learning of amplitude modulated tones in Mongolian gerbils. Neurosci. Lett. 261, 13-16 (1999).

21. H. Hessel et al., A method for the induction of a cochlea-specific auditory deprivation in the gerbil (Meriones unguiculatus). ORL J. Oto-Rhino-Laryngol. Its Relat. Spec. 60, 61-66 (1998).

22. W. Liu, R. L. Davis, Calretinin and calbindin distribution patterns specify subpopulations of type I and type II spiral ganglion neurons in postnatal murine cochlea: Heterogeneous distribution of calretinin and calbindin in sgn. J. Comp. Neurol. 522, 2299-2318 (2014).

23. R. Hallworth, R. F. Ludueña, Differential expression of beta tubulin isotypes in the adult gerbil cochlea. Hear. Res. 148, 161-172 (2000).

24. M. C. Liberman, Auditory-nerve response from cats raised in a low-noise chamber. J. Acoust. Soc. Am. 63, 442-455 (1978).

25. J. A. Cardin et al., Targeted optogenetic stimulation and recording of neurons in vivo using cell-type-specific expression of Channelrhodopsin-2. Nat. Protoc. 5, 247-254 (2010).

26. X. Han, In Vivo Application of Optogenetics for Neural Circuit Analysis. ACS Chem. Neurosci. 3, 577-584 (2012).

27. L. A. Johnson, C. C. Della Santina, X. Wang, Selective Neuronal Activation by Cochlear Implant Stimulation in Auditory Cortex of Awake Primate. J. Neurosci. 36, 12468-12484 (2016).

28. C. M. Zierhofer, I. J. Hochmair-Desoyer, E. S. Hochmair, Electronic design of a cochlear implant for multichannel high-rate pulsatile stimulation strategies. IEEE Trans. Rehabil. Eng. 3, 112-116 (1995).

29. C. Goßler et al., GaN-based micro-LED arrays on flexible substrates for optical cochlear implants. J. Phys. Appl. Phys. 47, 205401 (2014).

30. J. Bourien et al., Contribution of auditory nerve fibers to compound action potential of the auditory nerve. J. Neurophysiol. 112, 1025-1039 (2014).

31. J. M. Goldberg, P. B. Brown, Response of binaural neurons of dog superior olivary complex to dichotic tonal stimuli: some physiological mechanisms of sound localization. J. Neurophysiol. 32, 613-636 (1969). 
32. C. M. Hillery, P. M. Narins, Frequency and time domain comparison of low-frequency auditory fiber responses in two anuran amphibians. Hear. Res. 25, 233-248 (1987).

33. H. Thomas, J. Tillein, P. Heil, H. Scheich, Functional organization of auditory cortex in the mongolian gerbil (Meriones unguiculatus). I. Electrophysiological mapping of frequency representation and distinction of fields. Eur J Neurosci. 5, 882-897 (1993).

34. P. V. Watkins, D. L. Barbour, Rate-level responses in awake marmoset auditory cortex. Hear. Res. 275, 30-42 (2011).

35. C. Vogl et al., Tryptophan-rich basic protein (WRB) mediates insertion of the tail-anchored protein otoferlin and is required for hair cell exocytosis and hearing. EMBO J., e201593565 (2016). 36. C. A. Schneider, W. S. Rasband, K. W. Eliceiri, NIH Image to ImageJ: 25 years of image analysis. Nat. Methods. 9, 671-675 (2012).

37. W. van Aarle et al., The ASTRA Toolbox: A platform for advanced algorithm development in electron tomography. Ultramicroscopy. 157, 35-47 (2015).

38. W. van Aarle et al., Fast and flexible X-ray tomography using the ASTRA toolbox. Opt. Express. 24, 25129-25147 (2016).

39. P. V. D. Zee, Measurement and Modelling of the Optical Properties of Human Tissue in the near infrared. Med. Phys. 19, 1-302 (1992).

40. J. Steinbrink, Nahinfrarotspektroskopie am Kopf des Erwachsenen mit PikosekundenZeitaufloeosung, 157-158 (2000).

41. N. Yavari, S. Andersson-Engels, J. Sorensen Dam, J. Antonsson, K. Wardell, in Novel Optical Instrumentation for Biomedical Applications II (OSA, 2005; https://www.osapublishing.org/abstract.cfm?uri=ECBO-2005-MG4), pp. MG4-MG4.

42. N. Lue et al., Tissue refractometry using Hilbert phase microscopy. Opt. Lett. 32, 3522-4 (2007).

43. A. O. Ugnell, P. Å. Öberg, The optical properties of the cochlear bone. Med. Eng. Phys. 19, 630-636 (1997).

44. F. Bevilacqua et al., In vivo local determination of tissue optical properties: applications to human brain. Appl. Opt. 38, 4939-50 (1999).

45. M. H. Niemz, Laser-tissue interactions : fundamentals and applications (Springer, 2007).

46. S. L. Jacques, Optical properties of biological tissues: a review. Phys. Med. Biol. 58, R37R61 (2013).

47. S. Haleh, G. Hirac, P. Frédéric, Optical properties of mice skull bone in the 455- to 705-nm range. J. Biomed. Opt. 22, 10503 (2017). 
48. V. V. Tuchin, Tissue optics : light scattering methods and instruments for medical diagnosis (2007; http://spie.org/Publications/Book/2175698). 
Acknowledgments: We thank Dr. M. Rivolta for teaching us the modiolar injection. We thank K. Bodensiek for devising the optical model. We thank M. Töpperwien and T. Salditt for enabling the X-ray tomography. We thank Sandra Gerke, Jenny Paech, Christiane Senger-Freitag and Peter Wenig for expert technical assistance.

Funding: This work was funded by the European Research Council (ERC) under the European Union's Horizon 2020 research and innovation programme (grant agreement No 670759 advanced grant "OptoHear" to T.M. and was further supported by grants the German Research Foundation through the collaborative research center 889 (project research rotation of C.W.), the Center for Nanoscale Microscopy and Molecular Physiology of the Brain and the Leibniz program (to T.M.) and a grant of the Georgius Agricola Foundation Ruhr (to C.W. and Stefan Dazert). A.D. is a fellow of the German Academic Scholarship Foundation. C.V. is a Creutzfeldt Fellow of the Elisabeth and Helmut Uhl Foundation.

Author contributions: C.W., A.D., M.J. and T.M. designed the study. C.W. performed AAV injections, immunohistochemistry, o/aABR recordings and SGN recordings in the early phase of the project. C.W and M.J. performed recordings from single AC units. A.D. performed AAV injections, chronic $\mathrm{oCl}$, o/aABR recordings, shuttle-box and deafening experiments. A.D. and M.J. designed the behavioral experiments. A.H. performed AAV injections and recordings from single SGNs that are presented in this manuscript. D.K. performed X-ray tomography. C.J.D.A. performed Monte Carlo modeling of optical rays. C.V. performed immunohistochemistry of whole-mount organs of Corti. G.H. and M.J. designed the shuttle-box and other hard/software. All authors analyzed data and prepared the manuscript.

Competing interests: the authors declare no competing interests.

Data and material availability: The data that support the findings of this study is available from the corresponding author upon reasonable request. 


\section{Supplementary methods}

Animals

Experiments were performed in 131 male and female Mongolian gerbils (Meriones unguiculatus; 44 CatCh-positive; 52 CatCh-negative; 11 Chronos-negative; 5 PBS-injected and 19 wildtype). The exact number of animals contributing to each experiment is listed in table S1. Injections were performed on animals older than 8 weeks and for all other experiments animals were older than 12 weeks of age. All experiments were approved by the local animal care and use committee and the authorities of the State Lower Saxony.

Postnatal intracochlear injection of Adeno-Associated virus (AAVs)

Gerbils were anesthetized with isoflurane $(4 \%$ at $1 \mathrm{l} / \mathrm{min}$ for induction, $1-2 \%$ at $0.4 \mathrm{l} / \mathrm{min}$ for maintenance) during postnatal intramodiolar injections of AAVs and placed on a remotecontrolled heating blanket (Hugo Sachs Elektronik - Harvard Apparatus). Appropriate analgesia was achieved by subdermal injections of buprenorphine $(0.1 \mathrm{mg} / \mathrm{kg}$ bodyweight (BW)). Anesthetic depth was ensured by frequent testing of the absence of the hind-limb withdrawal reflex. Accordingly, the isoflurane concentration was adjusted. All following surgical steps were performed under microscopic control. The left middle ear was accessed by exposing and carefully opening the bulla using a retroauricular approach. A small hole was drilled into the basal modiolus using a KFlex dental file (no. 15) going through the upper circumfence of the round window niche, bypassing the scala tympani. The right ear was left untreated and served as a control in histological analyses. Approximately three microliters of Adeno-associated Virus solution $\left(3.2 \times 10^{12}\right.$ $-2.7 \times 10^{13}$ genome copies/ $\mu$ l) were injected into the modiolar center via quartz micropipettes (Science products, pulled on a P-2000 laser puller, Sutter Instruments; tip diameter $20 \mu \mathrm{m}$ ) connected to a pressure microinjector (100 - 125 PSI, PLI-100 pico-injector, Havard Apparatus). Control group received equal volume of AAV-free phosphate buffered saline (PBS). Retroauricular autologous connective tissue was used to cover the opened bulla and the surgical situs was closed by suturing the skin. All animals were allowed to recover for at least 4 weeks after injection.

Recording of Auditory Brainstem Responses (ABRs)

ABRs were recorded by needle electrodes inserted underneath the pinna, on the vertex and on the back near the tail of the animal. The potential difference between vertex and mastoid subdermal needles was amplified using a custom designed amplifier, sampled at a rate of $50 \mathrm{kHz}$ (NI PCl-6229, National Instruments), stored on a PC hard drive and filtered off-line $(300-3,000 \mathrm{~Hz}$ Butterworth filter) for acoustically and optically ABRs (a/oABRs). a/oABRs were carried out with 
the animal placed on a custom build heating plate (at $38^{\circ} \mathrm{C}$ ) on a vibration-isolation table within a soundproof chamber (Industrial Acoustics Company $\mathrm{GmbH}$ ). Gerbils were anesthetized with isoflurane ( $4 \%$ at $1 \mathrm{l} / \mathrm{min}$ for induction, $1-2 \%$ at $0.4 \mathrm{l} / \mathrm{min}$ for maintenance) and appropriate analgesia was obtained with subdermal injection of buprenorphine $(0.1 \mathrm{mg} / \mathrm{kg} B W)$. Stimulus generation and presentation (acoustic and optic) as well as data acquisition were realized by custom written software in MATLAB (The MathWorks, Inc.) employing National Instruments data acquisition (NI-DAQ) cards in combination with custom build hardware to amplify as well as to attenuate audio signals, control laser drivers and to acquire data. For aABRs clicks of $0.3 \mathrm{~ms}$ length were presented near field via a single loudspeaker (Vifa, Avisoft Bioacoustics) placed $30 \mathrm{~cm}$ in front of the animal at the level of the animal's head. Sound pressure levels were calibrated with a 0.25 inch microphone (D 4039, Brüel \& Kjaer GmbH) and measurement amplifier (2610, Brüel \& Kjaer $\mathrm{GmbH}$ ). For optical cochlear stimulation, a blue laser (473 nm, MLL-FN-473-100, $100 \mathrm{~mW}$ diode pumped solid state [DPSS]; Changchun New Industry Optoelectronics) was coupled to a 200 $\mu \mathrm{m}$ optical fiber that was inserted into the cochlea either through the round window or a cochleostomy performed in the middle cochlear turn. The left cochlea was approached as described for intracochlear injections. Laser power was calibrated before every experiment with a laser power meter placed at a distance of $1 \mathrm{~cm}$ from the fiber tip (Gentec-EO Solo 2). Due to the unknown exact position of the optical fiber used in each experiment with respect to the SGNs to be stimulated we cannot assess the exact irradiance. However, based on a Monte Carlo ray tracing model (fig. S9) we estimate the irradiance to not exceed $3 \mathrm{~mW} / \mathrm{mm}^{2}$ at the site of neural stimulation (Rosenthals' canal). The dynamic range of the stimulus intensity to $A B R$ amplitude relationship was given in $d B$ and defined as the range from threshold, identified by manual observation, to the point where either the slope of the stimulus-amplitude function is less than or equal to $5 \%$ of the maximum slope or the maximum intensity employed. To compare the change in ABR amplitude and latency in dependence of stimulus repetition rates (Fig. 2) ABR data was normalized for each individual animal. For ABR amplitude, $\mathrm{p} 1-\mathrm{n} 1$ amplitudes in response to all stimulus conditions were divided by the $\mathrm{p} 1-\mathrm{n} 1$ amplitude of the $10 \mathrm{~Hz}$ stimulation rate condition (slowest stimulation rate tested). To normalize ABR latency (Fig. 2M), the p1 latency of the $10 \mathrm{~Hz}$ stimulation rate condition was subtracted from $\mathrm{p} 1$ latencies of all stimulus conditions. This was done to compensate for the different latencies observed in aABRs vs oABRs.

\section{Extracellular single auditory nerve fiber recordings}

Gerbils were anesthetized with Isoflurane $(4 \%$ at $1 \mathrm{l} / \mathrm{min}$ for induction, $1-2 \%$ at $0.4 \mathrm{l} / \mathrm{min}$ for maintenance) and appropriate analgesia was obtained with subdermal injection of buprenorphine 
$(0.1 \mathrm{mg} / \mathrm{kg} \mathrm{BW})$. The left auditory nerve was approached by removing parts of the left occipital bone and carefully aspirating the left hemisphere of the cerebellum (for more detail see (30)). Optical stimulation of the cochlea was performed as described for OABRs. During recordings in the auditory nerve the animal was placed on a heating plate (custom made, body temperature kept at $38{ }^{\circ} \mathrm{C}$ rectal temperature) on a vibration-isolation table within a heated $\left(30{ }^{\circ} \mathrm{C}\right)$ soundproof chamber (Industrial Acoustics Company $\mathrm{GmbH}$ ). The animal's head was fixed by a head post mounted with dental cement to the skull at the vertex. Pulled (P-1000, Sutter Instrument) glass micro electrodes (borosilicate, 1B100 F-4, world precision instruments) filled with $3 \mathrm{M} \mathrm{NaCl}(70-$ $80 \mathrm{M} \Omega$ tip resistance) were advanced through the auditory nerve by a single-axis inchworm micromanipulator (Burleigh EXFO 8200). Extracellular signals were amplified (ELC-03XS amplifier, NPI Electronics), band-pass filtered between 300 and $3000 \mathrm{~Hz}$ and digitized (National instruments card PCle-6323) in custom written software (MATLAB, The MathWorks, Inc.). Phase-locking was quantified using the vector strength (31) according to the following equation : vector strength $=\frac{\sqrt{\left[\sum_{i=1}^{n} \cos \theta_{i}\right]^{2}+\left[\sum_{i=1}^{n} \sin \theta_{i}\right]^{2}}}{n}$, where $\theta_{1}, \theta_{2}, \theta_{3}, \ldots, \theta_{\mathrm{n}}$ are phases from spikes. This parameter varies from 0 to 1 . The significance of phase-locking was tested with the Rayleigh test of circular data (32). The presence of two subpopulations was investigated by a k-means clustering analysis using Akaike's Information Criterion (criterion $=1.5 \mathrm{spike} / \mathrm{pulse}$ ). In order to distinguish tonic vs phasic responses, we calculated an adaptation ratio as the ratio between the discharge rate during the first $50 \mathrm{~ms}$ and $900 \mathrm{~ms}$ for repetition rate $\geq 100 \mathrm{~Hz}$ ).

\section{Chronic oCl}

Anesthesia and analgesia were administered identical as for acute oABR recordings. An optical fiber (Thorlabs; $200 \mu \mathrm{m}$ diameter, $30 \mathrm{~mm}$ length) coupled to a $2.5 \mathrm{~mm}$ steel ferrule was funneled through a custom bent stainless steel capillary $(500 \mu \mathrm{m}$ inner diameter; bent at the minimal longterm bending radius of the optical fiber, i.e. $24 \mathrm{~mm}$; ca. $29 \mathrm{~mm}$ length) for optimal shape and protection of the fiber. The skin overlying the parietal bones was removed and a fiber-holder (aluminum, $30 \mathrm{~mm}$ length) was fixed to the head of the animal using a self-etching UV-glue and dental cement. A blank metal wire $(500 \mu \mathrm{m}$ diameter; impedance $<1 \Omega)$ was implanted just left to the midline of the animal's skull to serve as negative electrode for chronic oABR measurements. Care was taken to avoid touching the aluminum fiber-holder with the metal wire. Needle electrodes underneath the pinna and on the back of the animal were placed acutely whenever oABRs were recorded (recording procedure: see ABR recordings). The fiber implant was then placed in the round window of the animal (using the retroauricular approach described above) 
while oABRs in response to blue light test pulses were constantly monitored online (12). As soon as a fiber position was found that reliably elicited oABRs, the implant was fixed at the cochlea of the animal using dental cement. For a second point of stabilization, the ferrule-coupled end of the implant was glued to the fiber-holder on the animal's head. Finally, the skin of the animal was sutured and glued with histoacrylic glue. Animals were given subcutaneous injections of Carprofen $(0.05 \mathrm{mg} / 10 \mathrm{~g} \mathrm{BW})$ immediately, $24 \mathrm{~h}$ and $48 \mathrm{~h}$ after the surgery. OABRs were then regularly measured under isoflurane anesthesia during the course of behavioral testing to confirm the presence of a physiological response.

\section{Shuttle box behavior}

For behavioral testing, a custom-built shuttle box located in a sound attenuated chamber (75x50x100 cm (WxDxH); IAC Acoustics) was used. The shuttle box consists of two platforms $(25 \times 25 \mathrm{~cm}$; each assembled from 16 metal bars of $4 \mathrm{~mm}$ diameter, $15 \mathrm{~mm}$ spacing center to center), separated by a hurdle $(4 \mathrm{~cm}$ height) and surrounded by plastic tubes $(2.5 \mathrm{~cm}$ diameter, spaced $3.5 \mathrm{~cm}$ center to center; $35 \mathrm{~cm}$ height). Both platforms were mounted on an individual set of metal springs (4 per platform) and equipped with acceleration sensors (LIS344ALH; STMicroelectronics) to determine the position of the animal (fig. S10). Generation and presentation of stimuli as well as data acquisition was done using custom-written Matlab-scripts (The MathWorks, Inc) actuating NI-DAQ cards combined with custom build hardware for signal attenuation and amplification. Acoustic stimuli were delivered using a loudspeaker (ScanSpeak, Avisoft Bioacoustics) centered over the shuttle box and mounted to the ceiling of the chamber. For optogenetic stimulation, a blue laser (488 nm, LBX-488-100-CSB, Oxxius) was coupled to a $200 \mu \mathrm{m}$ optical fiber with an integrated rotary joint (Thorlabs) mounted to the ceiling of the chamber. The free end of the fiber could then be connected to the ferrule end of the animal's implant to optically stimulate spiral ganglion neurons. For behavioral training, animals were placed in the shuttle box and given an adaptation time of 5 minutes before stimuli were presented. Both acoustic and optical stimuli (click or pulse trains) had durations of $250 \mathrm{~ms}$ and were presented with a repetition rate of $2 \mathrm{~Hz}$ during a response window of 6 seconds (60-80 trials per session with an inter-trial-interval randomized between 16 and 21 seconds). Upon perception of a stimulus the animal was expected to cross the hurdle of the shuttle box. If the animal crossed the hurdle within the response window, the trial was considered a hit and the stimulus was switched off. If the animal did not cross the hurdle in time, an aversive electric stimulus (0.2$2 \mathrm{~mA}$ ) was administered to the animal's feet via the metal bars of the platform until the animal crossed the hurdle (or for a maximum of 6 seconds) and the trial was considered a miss. Per 
session, 10 sham trials in which no stimulus was presented and accordingly no consequences for the reaction of the animal existed were randomly intermixed with regular target trials in order to determine the baseline activity of the animal. The hit (sham) rate of the animal for target (and sham) trials is reported as the fraction of target (or sham) trials in which the animal crossed the hurdle divided by the total number of target (or sham) trials.

In the morning of the second day after fiber implantation animals were placed in the shuttle box and underwent one session of training using acoustic stimulation (250 ms click trains at $2 \mathrm{~s}^{-1}$ with a click rate of $50 \mathrm{~s}^{-1}$; peak intensity: $91.5 \mathrm{~dB}$ SPL; calibrated with a 0.25 inch microphone [D 4039, Brüel \& Kjaer $\mathrm{GmbH}$ ] and measurement amplifier [2610, Brüel \& Kjaer $\mathrm{GmbH}$ ]) as cues for hurdle crossing. This training was necessary because after being placed in the shuttle box and receiving electrodermal stimulation for the first time, previously naïve animals showed unspecific locomotion (e.g. undirected, repetitive jumping or quick spurts across the hurdle) which was abandoned after a few trials. Thus, to avoid damage of the implant or injury of the animal, this session served to habituate animals to the setup. In the afternoon of the same day and of the five subsequent days animals underwent behavioral training using supra-threshold optogenetic stimulation ( $250 \mathrm{~ms}$ pulse trains with a repetition rate of $2 \mathrm{~s}^{-1}\left(10 \mathrm{~ms}\right.$ pulses at $50 \mathrm{~s}^{-1}, 25-30 \mathrm{~mW}$ at $472 \mathrm{~nm}$ ). In all sessions employing optogenetic stimulation animals were briefly anesthetized with isoflurane in order to connect the implant to the laser-coupled optical fiber for light delivery (adaptation time started once the animals were active again). In session no. 7 the laser beam path was blocked between the implant and the optical fiber in order to ensure that animals did not use any cues (especially visual) other than optogenetic stimulation to solve the task. Once the animals learned the task, behavioral thresholds of optogenetic stimulation were determined. For this, the stimulus structure of $250 \mathrm{~ms}$ pulse trains presented with a repetition rate of $2 \mathrm{~s}^{-1}$ was similar to the other sessions and pulses had $1 \mathrm{~ms}$ duration, a repetition rate of $10 \mathrm{~s}^{-1}$ and varying amplitude or varying pulse durations, a repetition rate of $2 \mathrm{~s}^{-1}$ and $25 \mathrm{~mW}$ intensity to determine amplitude or pulse duration thresholds, respectively. The repetition rate for pulse duration threshold determination was chosen to be lower in order to avoid cumulative effects of several pulses that would lead to a percept. In each session animals absolved 10 trials of each condition as well as 10 sham trials and response rates were averaged across sessions. After determining behavioral thresholds, 3 sessions using acoustic stimulation (identical to the very first habituation session) were absolved to check whether animals, once they learned the task using optogenetic cues, can generalize these to acoustic stimuli. Finally, the control experiment in which the laser beam path was blocked was repeated to rule out that animals, after being trained on harder tasks 
(thresholds) and generalizing the cues to acoustic stimulation will be able to use any kind of visual cue that might potentially originate from the laser stimulation.

Recordings from primary auditory cortex neurons

Recordings in the primary auditory cortex (AI) were performed in the same environment and conditions as described for ABR recordings. In addition, the animal's head was fixed by a head post mounted with dental cement to the skull at the vertex. The bone above field Al of the right auditory cortex - contralateral to the intracochlear injection - was removed, leaving the dura intact. Multi- as well as single-unit recordings were realized by single tungsten electrodes ( $5 \mathrm{M} \Omega$ ) positioned and advanced by a LN Junior 4RE micro manipulator (Luigs\&Neumann). Amplification of signals, data acquisition as well as extracellular spike detection were implemented by a Neuralynx system (Digital Lynx 4S, Cheetah data acquisition hard and software, Neuralynx Inc.). For local field potentials (LFP) neuronal signals were bandpass filtered in a range of $0.1-300 \mathrm{~Hz}$, for neuronal spikes between 600 and $6,000 \mathrm{~Hz}$ and after amplification recorded at a sampling rate of $32 \mathrm{kHz}$. Data analysis was performed off-line with custom software written in MATLAB (The MathWorks, Inc.) and Python (Python Software Foundation). Stimulus generation and presentation was obtained using the same hardware as described for ABR recordings also driven by custom written MATLAB software (The MathWorks, Inc.). Before driving a tangential, dorsal-toventral track through Al - aiming at layer IV in $600 \mu \mathrm{m}$ depth (33) - location and tonotopy of AI was determined by recording LFPs from several positions at the cortical surface while playing acoustic tone bursts from $0.25-32 \mathrm{kHz}$ at $60-90 \mathrm{~dB}$ SPL in 9 logarithmic steps. Search stimuli for single unit activity consisted of acoustic sine bursts covering $0.25-32 \mathrm{kHz}$ at $60-90 \mathrm{~dB} \mathrm{SPL}$ in quarter octave steps. However, when encountering spontaneously firing neurons, we always attempted to drive them with auditory stimuli and/or light pulses. For neurons which were driven by light pulses we calculated a monotonicity index as the evoked firing rate at the highest light intensity divided by the maximum evoked firing rate (34). Neurons with a monotonicity index of 0.5 or lower were considered "nonmonotonic" (34).

\section{Deafening}

Using the retroauricular approach as described for the virus injections, gerbils were deafened by bilateral intracochlear injections of 2-3 $\mu \mathrm{l}$ Kanamycin solution through the round window membrane $(100 \mathrm{mg} / \mathrm{ml}$, Kanamysel, Selectavet). After the injection, a small piece of gelatine sponge soaked in Kanamycin solution was placed in front of the round window (Gelita-Spon; Gelita medical) and the surgical site was covered with connective tissue. Animals were allowed to 
recover for 7-10 days before deafness was confirmed by ABR recordings as well as shuttle box behavior.

Histology

Cochlear cryosections: Cochleae of both sides were taken out of the temporal bone immediately after death of the animal and fixed in $4 \%$ paraformaldehyde (PFA) for $1 \mathrm{~h}$. For cryosectioning cochleae were decalcified in Ethylenediaminetetraacetic acid (EDTA, $0.12 \mathrm{M}$ for $3-4$ days). Immunolabeling of calretinin, neurofilament and CatCh-eGFP was performed on $16 \mu \mathrm{m}$ thin sections with calretinin CG1 (1:300, goat, Swant), Neurofilament 200 (1:300, mouse, Sigma) and Alexa Fluor 488-conjugated GFP (1:500, rabbit, Invitrogen) antibodies, respectively, and appropriate secondary antibodies (1:300, Invitrogen) in the case of calretinin and NF200. Images were acquired using a Leica SP5 confocal microscope.

Whole-mount organ of Corti preparations: After decapitation, cochleae from adult wildtypecontrol and pharmacologically-deafened gerbils were dissected, fixed in $4 \%$ formaldehyde solution (overnight, $4^{\circ} \mathrm{C}$ ) and decalcified in $0.12 \mathrm{M}$ EDTA solution $\left(10-14\right.$ days at $4^{\circ} \mathrm{C}$ ). Subsequently, organs of Corti were dissected in phosphate-buffered saline (PBS) and immunostainings performed essentially as described in Vogl, Panou, et al. 2016 (35). Briefly, specimens were incubated with goat serum dilution buffer (comprised of: 16\% normal goat serum, $450 \mathrm{mM} \mathrm{NaCl}, 0.3 \%$ Triton X-100, $20 \mathrm{mM}$ phosphate buffer, $\mathrm{pH}$ 7.4) to block non-specific binding sites and finally probed with a rabbit anti-calretinin primary antibody (1:300; Swant, CR7697). For immunolocalization, an Alexa Fluor 568-conjugated goat anti-rabbit secondary antibody (Thermo Fisher Scientific, A11011) was combined with an Alexa Fluor 633-coupled phalloidin (Thermo Fisher Scientific, A22284) to co-label F-actin-containing substructures within the sensory epithelium, such as hair cell stereocilia and cuticular plates, as well as the actincytoskeleton of adjacent supporting cells. Finally, specimens were mounted in Mowiol mounting medium and imaged either on an (i) Axio Imager M2 System (5x objective, Zeiss) controlled by a Neurolucida system (Version 11, MBF Bioscience) or (ii) a Leica TCS SP2 laser-scanning confocal microscope with a 1.4 NA 63x oil immersion objective and HeNe $561 \mathrm{~nm}$ and $633 \mathrm{~nm}$ excitation laser lines. Images were processed for display in ImageJ (36) and Adobe Illustrator and presented as maximum projections of the acquired image stacks.

\section{X-Ray tomography}

After behavioral experiments the placement of the optical fiber was assessed with phase-contrast tomography (19). The customized cone-beam in-line tomography instrument was equipped with a 
liquid-metal x-ray source (JXS D2, Excillum) operated at $70 \mathrm{kV}$ acceleration voltage and combined with a $20 \mu \mathrm{m}$ LuAG-scintillator-based fiber-coupled charge-coupled device (CCD) detector (6.5 $\mu \mathrm{m}$ pixel size, C12849-102U, Hamamatsu). The raw image data were fast Fourier-based transformed and finally reconstructed using the ASTRA toolbox $(37,38)$. Segmentation and 3D visualization of the reconstructed volume was realized with Avizo 3D 9 (Visualization Sciences Group, FEI Company). Automatic histogram-based segmentation was used to visualize bony structures, steel capillary and optical fiber. The basilar membrane and Rosenthal's canal were traced with semiautomatic tools.

\section{Monte Carlo ray tracing}

To estimate the light spread in the gerbil cochlea, we conducted Monte Carlo ray tracing simulation using TracePro ${ }^{\circledR}$ Standard 7.8.1 (Lambda Research Corporation). The following cochlear structures were included and modelled as meshes of 7500 triangles reconstructed from $x$-ray tomography: 1) scala tympani, 2) scala vestibuli and scala media, 3) modiolus and 4) Rosenthal's canal and peripheral processes. These volumes were embedded in a solid square prism to account for the cochlear bone. Mean optical properties for cerebrospinal fluid, brain tissue and bone taken from the literature were assigned to each mesh (39-48). The light sources were modeled as grid sources with a circular ray pattern of 1001 rings (3003001 rays, $\lambda=473 \mathrm{~nm}$ ) with a symmetric Gaussian spatial and angular beam distribution and uniform total intensity of $10 \mathrm{~mW}$.

Two different light sources were modelled: the optical fiber used in the experiments (Thorlabs FT200UMT, 0.39 NA) and an optimal emitter (here approximated by the profile of the Thorlabs FG010LDA, 0.1 NA optical fiber). The properties of the optical fiber used in the experiments were defined in TracePro as follows: grid boundary radius: $100 \mu \mathrm{m}$; waist radius of Gaussian beam profile: $100 \mu \mathrm{m}$; half angle of angular profile of the beam: $16.79^{\circ}$. The properties of the optimal emitter were defined as follows: grid boundary radius: $5 \mu \mathrm{m}$; waist radius of spatial profile: $5 \mu \mathrm{m}$; half angle of angular profile: $4.25^{\circ}$. The half angle for angular profiles were calculated using the formula: $\theta=\sin ^{-1}\left(N_{\text {fiber }} / \mathrm{n}_{\text {scala Tympani }}\right)$ ( $\theta$ : half angle; $N A$, numerical aperture; $n$, refractive index). The surface of the experimental fiber was placed according to the model obtained from $x$-ray tomography, the optimal emitter was placed on the center line of scala tympani, facing Rosenthal's canal at different tonotopic positions.

Radiant flux was read from 300 query points (included as solid spheres with a $5 \mu \mathrm{m}$ radius, with assigned optical properties of brain tissue) placed along the centerline of the Rosenthal's canal using custom scripts. Irradiance was calculated as: Irradiance $=$ Radiant Flux $/ 4^{*}$ pi*radius ${ }^{2}$. Irradiance values were scaled for the different intensities using the ratio between the simulation 
intensity $(10 \mathrm{~mW})$ and the other investigated intensities $(1,5$, and $30 \mathrm{~mW})$ assuming a linear relationship (confirmed by initial simulations for all intensities). Intensities of the optimal sources were scaled so their maximal irradiance (peak of the profile) at the center of Rosenthal's canal was equal to the maximal irradiance (peak of the profile) of the experimental fiber achieved with $10 \mathrm{~mW}$, and then scaled for the other intensities. Note that the required intensity of the optimal source was approximately 3 orders of magnitude lower than for the experimental fiber.

Smoothed traces from the experiment fiber were obtained from the average between the higher and lower envelopes of the traces calculated by spline interpolation over local maxima separated by at least 5 points. These smoothed traces were used to determine the irradiance threshold (taken as the irradiance value at $10 \mathrm{kHz}$ - the tonotopic position with the lowest auditory cortex response thresholds collected from the in vivo experiments - obtained with a total source intensity of $1 \mathrm{~mW}$ ) and the suprathreshold frequency ranges/octaves. All the data processing and analysis were done in MATLAB R2016a (The Mathworks, Inc) with custom scripts. 


\section{Supplementary Figures}

A

animal

AAV-construct

GIM1 AAV2/6-hSyn1-Chronos-eGFP

GIM2 AAV2/6-hSyn1-Chronos-eGFP

GIM3 AAV2/6-hSyn1-Chronos-eGFP

GIM4 AAV2/6-hSyn1-Chronos-eGFP

GIM5 AAV2/6-hSyn1-Chronos-eGFP

GIM6 AAV2/6-hSyn1-Chronos-eGFP

GIM7 AAV2/6-hSyn1-Chronos-eGFP

GIM8 AAV2/6-hSyn1-Chronos-eGFP

transfection rate [\%]

GST1 AAV2/6-hSyn1-Chronos-eGFP scala tympani GST2 AAV2/6-hSyn1-Chronos-eGFP scala tympani GST3 AAV2/6-hSyn1-Chronos-eGFP scala tympani

B
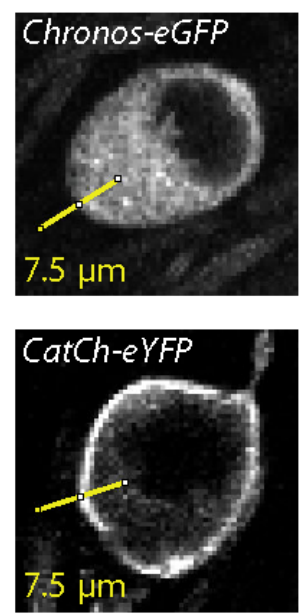

C

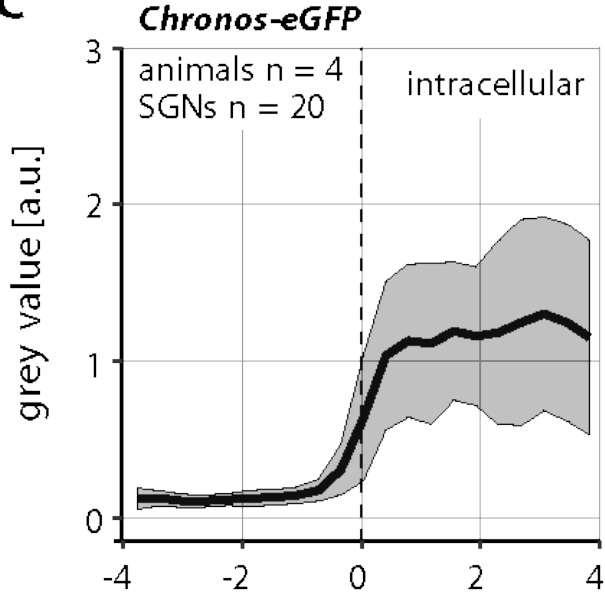

\section{injection}

intramodiolar intramodiolar intramodiolar intramodiolar intramodiolar intramodiolar intramodiolar intramodiolar apical middle basal

$\begin{array}{lll}66.67 & 62.07 & 84.00\end{array}$

$\begin{array}{llr}16.48 & 11.76 & 0.00\end{array}$

$\begin{array}{lll}70.24 & 56.67 & 83.33\end{array}$

$\begin{array}{lll}0.00 & 0.00 & 0.00\end{array}$

$\begin{array}{lll}1.52 & 3.70 & 0.00\end{array}$

$\begin{array}{lll}74.58 & 81.62 \quad 84.00\end{array}$

$\begin{array}{lll}0.00 & 0.00 & 0.00\end{array}$

$\begin{array}{lll}1.04 & 0.00 & 0.00\end{array}$

mean: $28.82 \quad 26.98 \quad 31.42$

\begin{tabular}{|c|c|c|c|c|}
\hline & 2.22 & 0.00 & 2.94 & negative \\
\hline & 0.00 & 0.00 & 0.00 & negative \\
\hline & 0.00 & 0.00 & 0.00 & negative \\
\hline mean: & 0.74 & 0.00 & 0.98 & \\
\hline
\end{tabular}

oABR

negative negative negative negative negative negative negative negative

\section{CatCh-eYFP}

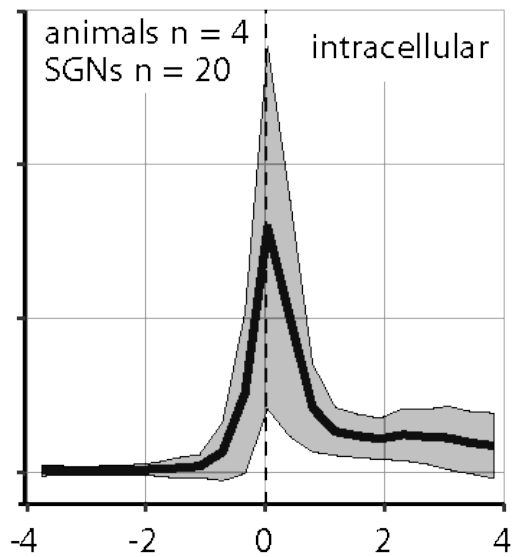

distance from cell membrane $[\mu \mathrm{m}]$

Figure S1. Effects of postnatal transfection of SGNs in adult Mongolian gerbils using the opsins Chronos and CatCh.

(A) Table of experiments summarizing the attempts to functionally express Chronos in SGNs of adult gerbils. (B) Comparative analysis of expression of Chronos vs. CatCh via immunohistology. A single line $7.5 \mu \mathrm{m}$ long line was centered on the presumed cell membrane such that half of the line was inside the SGN while excluding the nucleus and the other half was located in the extracellular space sparing neighboring cells. (C) Quantification of the grey value as a proxy for expression strength along the line profile for randomly chosen transduced SGNs (animals: $n=4$, cells: $n=20$ ). 
A

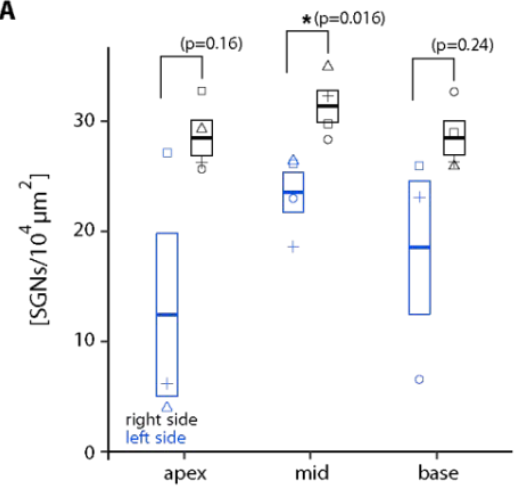

B

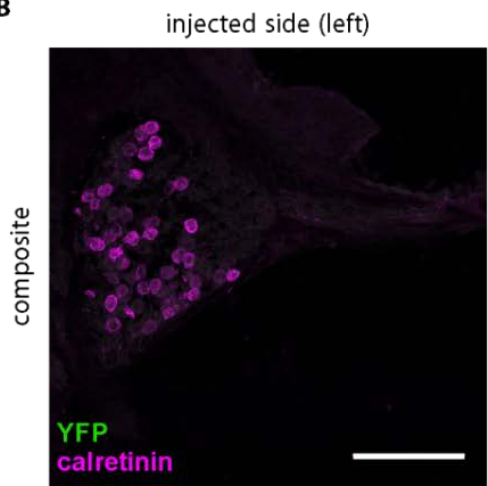

non-injected side (right)

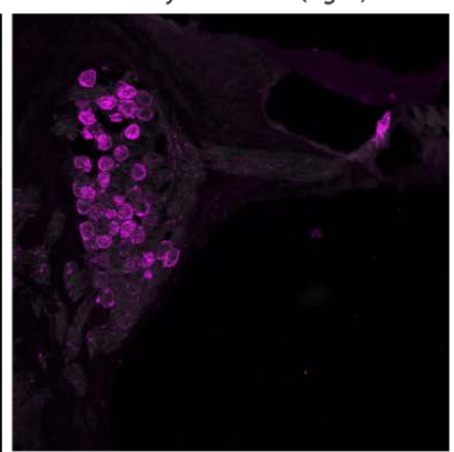

Figure S2. Spiral ganglion neuron (SGN) density of AAV-injected but CatCh-negative animals. (A) Quantification of SGN density in sections of the apical, middle and basal cochlear turn. Crossbars show mean and SEM, symbols raw data for investigated animals $(n=4)$. Statisical analysis was performed with a standard tow sample t-test. (B) Cochlear immunostainings (exemplary section of a middle turn) in maximum projection with immunofluorescence for YFP (green, no positive SGNs were observed, hence "CatCh-negative") and calretinin (staining SGNs, magenta), scale bar: $100 \mu \mathrm{m}$. 

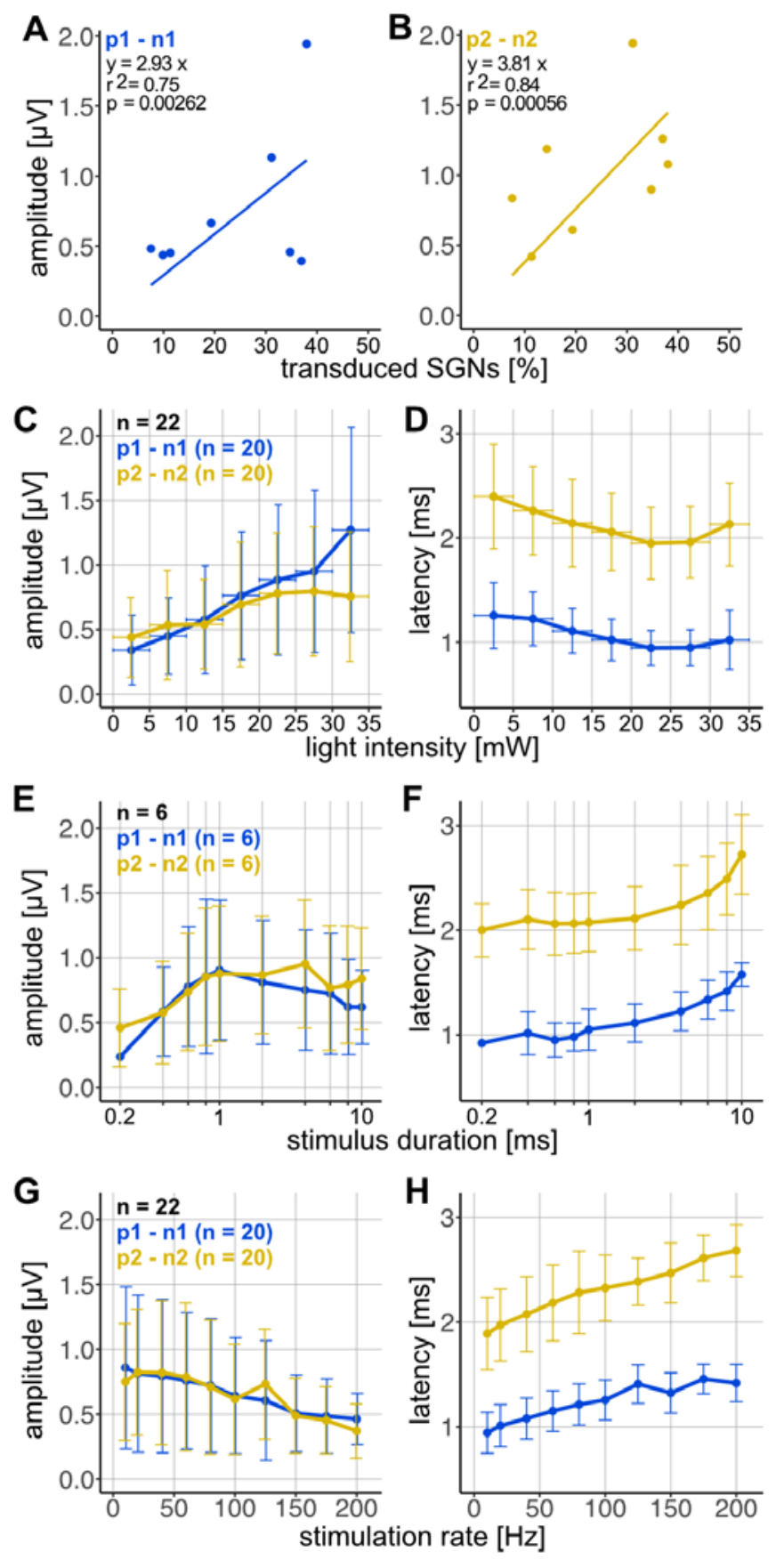

Figure S3. Optical ABRs (OABRs): dependence on stimulus intensity, rate and duration - relationship to fraction of CatChexpressing SGNs. (A, B) relationship of oABR amplitude for $p 1-n 1$ (A) and p2-n2 (B) and percentage of transduced SGNs analyzed by simple linear regression $(n=8)$. (C, D) Mean amplitude (C) and latency (D) as a function of light intensity (1 ms duration at $10 \mathrm{~Hz}$ ). $(E, F)$ Mean amplitude (E) and latency (F) as a function of stimulus duration $(16.3 \mathrm{~mW}$ at $10 \mathrm{~Hz}) .(\mathrm{G}, \mathrm{H})$ Mean amplitude (G) and latency $(\mathrm{H})$ as a function of stimulation rate $(21-32 \mathrm{~mW}$ for $1 \mathrm{~ms})$. Vertical error bars in C, D, E, F, G and H indicate SD, horizontal error bars in $C$ and $D$ represent the area of binned light intensities for calculating the mean and SD. 
A

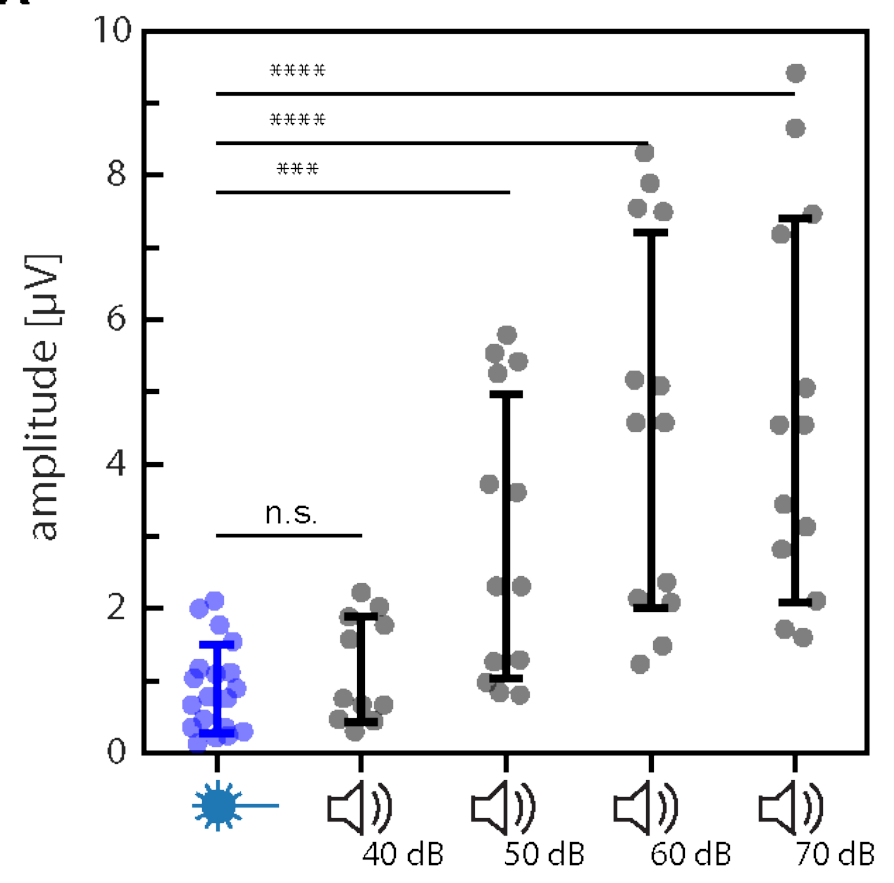

B

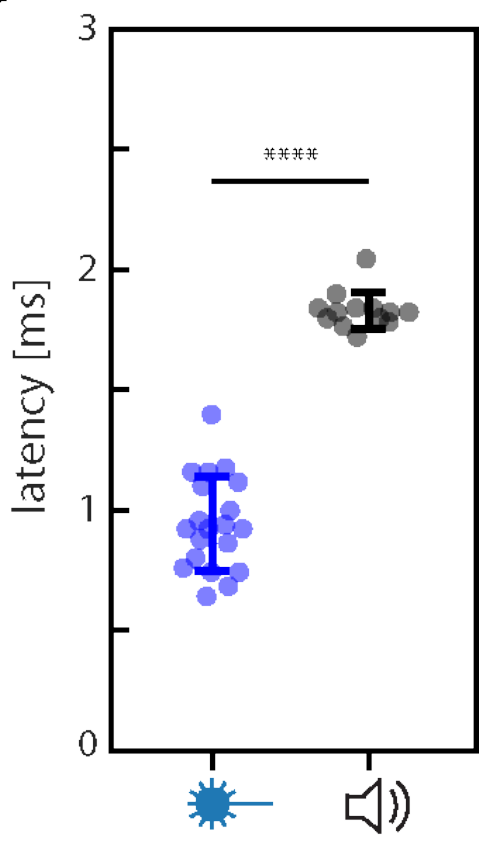

Figure S4. Amplitude and latency comparison between acoustically and optically evoked auditory brainstem

responses. (A) p1-n1 amplitudes evoked by optical stimulation with an intensity of $\sim 30 \mathrm{~mW}$ in AAV-CatCh injected gerbils (blue) or by acoustic clicks of different intensity in wildtype gerbils (black). Errorbars indicate mean \pm SD (optical: $n=19 ; 40 \mathrm{~dB}$ click: $n=11$; all other clicks: $n=13$ ). (B) Latency of the first wave (p1) evoked by optical stimulation with an intensity of $\sim 30 \mathrm{~mW}$ in AAV-CatCh injected animals (blue) or by acoustic clicks of $80 \mathrm{~dB}$ in wildtype gerbils (black). Errorbars indicate mean \pm SD (optical: $n=20$; click: $n=13$ ). Stars indicate significance $(*: p<0.05 ; * *: p<0.01 ; * * *: p<$ $0.001 ; * * *: p<0.0001)$. 
A

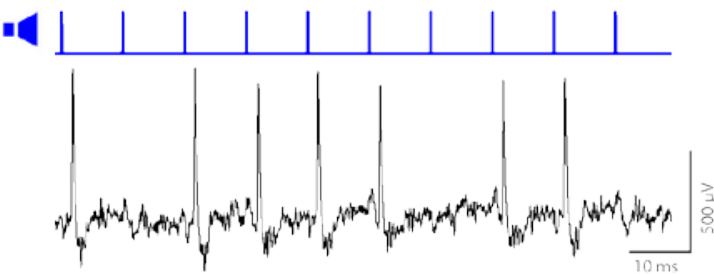

C

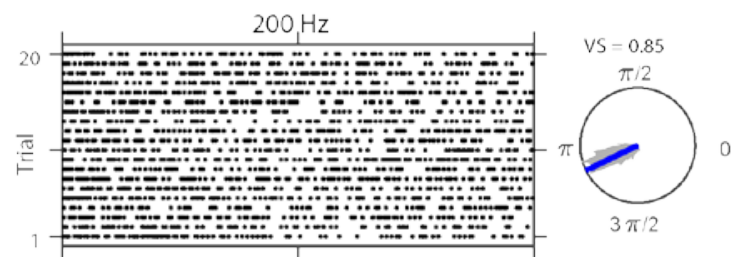

E

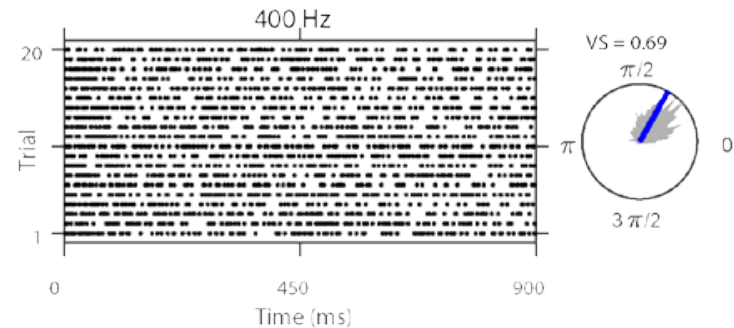

G

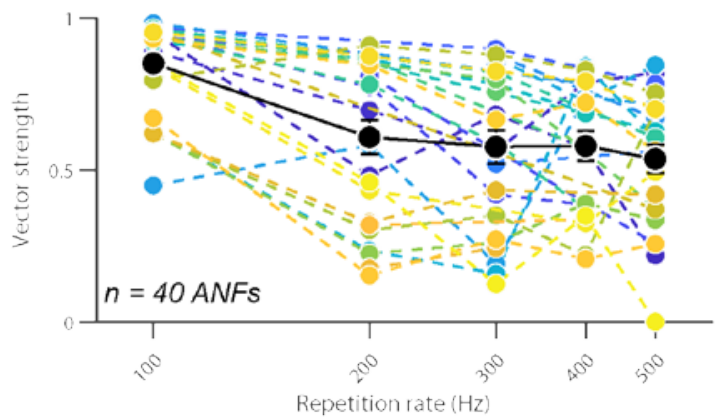

B

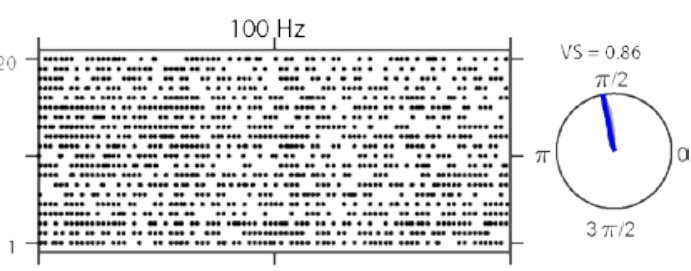

D

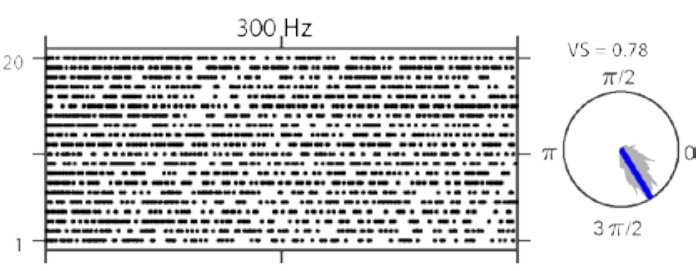

F
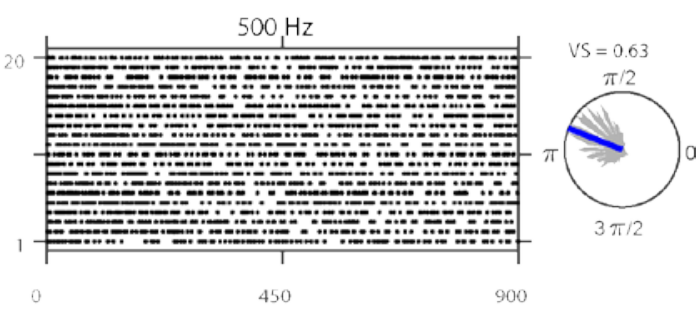

Time (ms)

H

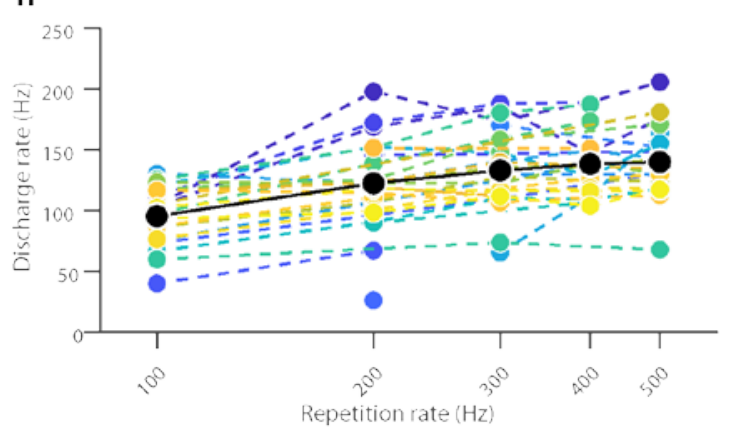

Figure S5 Single auditory nerve fiber (ANF) responses to acoustic click trains. (A) Exemplary unfiltered extracellular spikes of an ANF evoked by $0.1 \mathrm{~ms}$ acoustic clicks ( $20 \mathrm{~dB}$ above threshold). Blue trace corresponds to the acoustic stimuli. (B-F) Raster plots in response to 900 ms acoustic click trains at 5 different repetition rates (100, 200, 300, 400 and $500 \mathrm{~Hz}$ ). Panels to the right side of each raster plot show polar plots depicting the occurrence of spikes over the stimulation cycle. $(G, H)$ Vector strength $(G)$ and discharge rate $(H)$ as a function of repetition rate for 40 units from 6 gerbils. Different marker colors represent values from different units. Black traces represent the mean \pm SEM. 

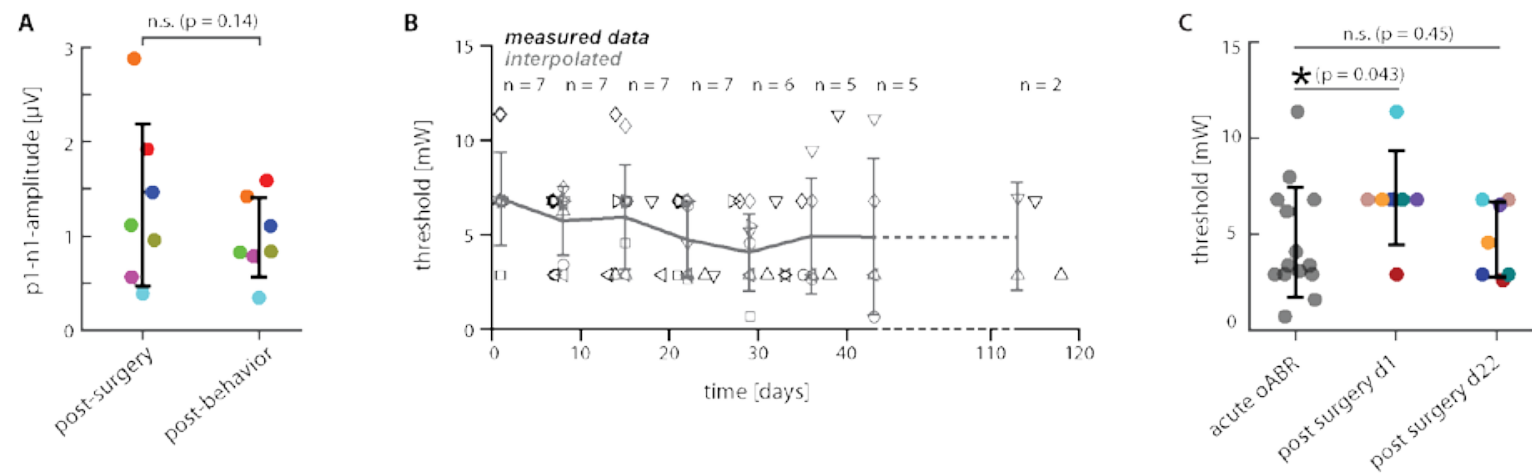

Figure S6. OABR amplitudes and thresholds in chronically implanted gerbils. (A) p1-n1 amplitudes in chronically implanted animals on the day after surgery and on the last day of behavioral testing. Error bars indicate mean $\pm S D$, colors mark individual animals $(n=7)$. (B) Thresholds of chronically implanted animals over time. Black markers are actually measured thresholds; grey markers are interpolated values for averaging. Solid lines indicate mean thresholds \pm SD across all animals. (C) Thresholds of oABRs recorded in acute experiments (grey; $n=14$ ) vs oABR thresholds of chronically implanted animals on the day after surgery and 3 weeks after surgery. A significant increase in threshold of implanted animals compared to animals from acute experiments was observed on the day after surgery, but vanished 3 weeks after surgery. 


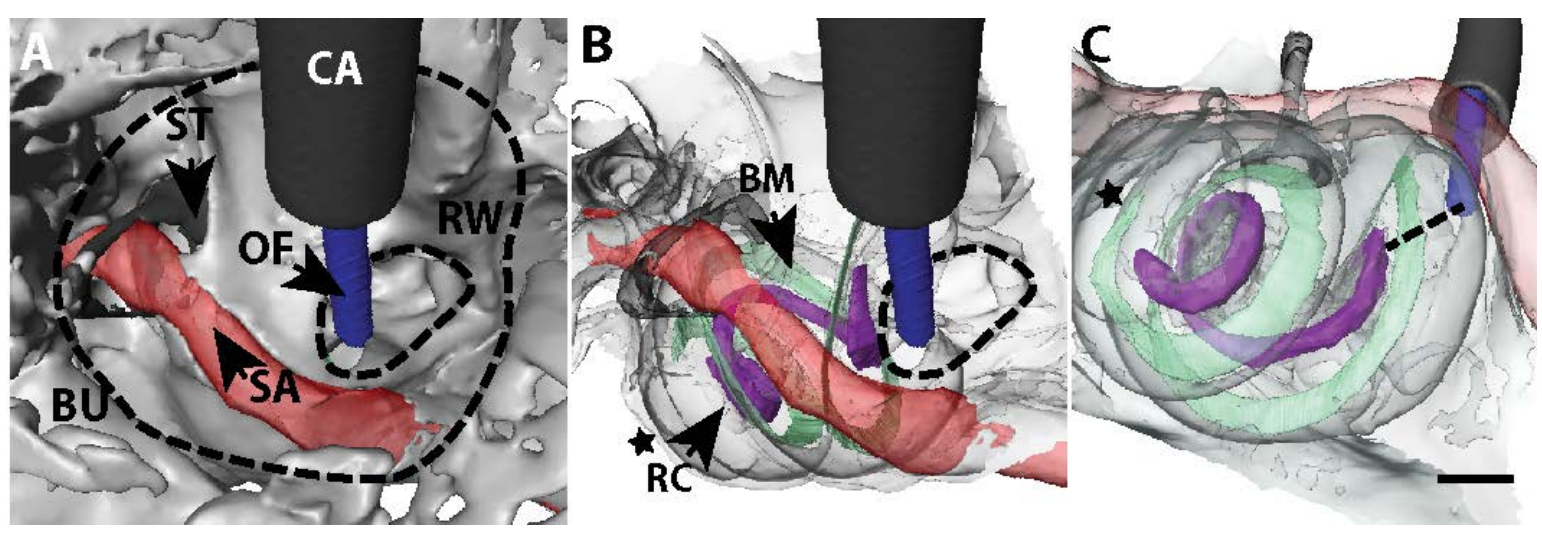

Figure S7. Determination of fiber position with 3D X-ray tomography. (A) Reconstructed ear showing the opened bulla (bone edge, BU) and the landmarks stapedial artery (SA) and stapes (ST). Optical fiber (OF, blue) housed in a stainless steel capillary (CA) placed in the round window (RW). (B) Cochlea rendered transparent reveals basilar membrane (BM), Rosenthal's canal (RC) and cochlear apex (star). (C) Distance from fiber tip to Rosenthal's canal is about $750 \mu \mathrm{m}$ with an angle of $34^{\circ}$ which corresponds to the numerical aperture of the optical fiber, scale bar: $500 \mu \mathrm{m}$. 


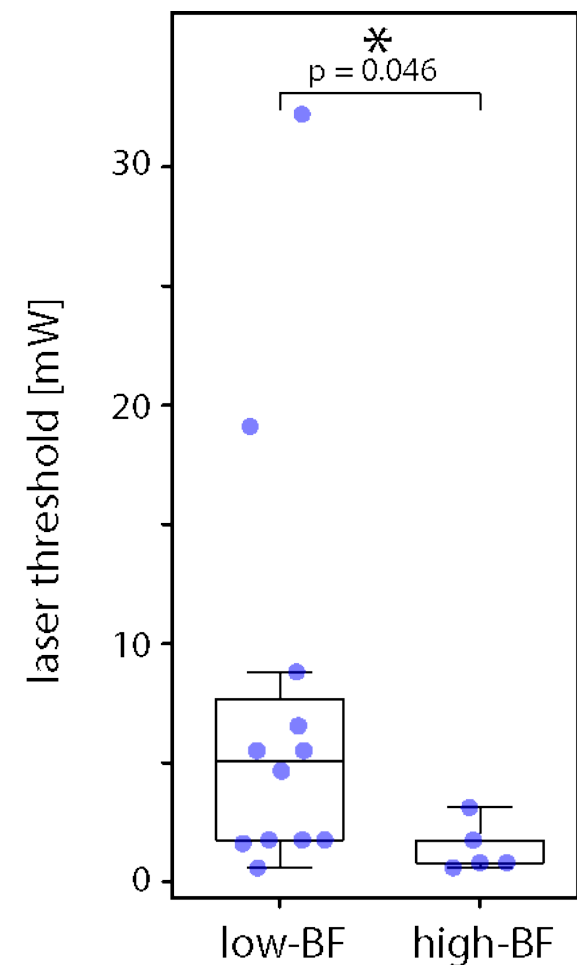

Figure S8. Optical thresholds for Al single units with high versus low best frequencies. Box plots show laser threshold of single units that could be driven by acoustic as well as optical stimuli. Single units are grouped in units with low $(1-8 \mathrm{kHz})$ and high best frequencies $(8-32 \mathrm{kHz})$. 
A

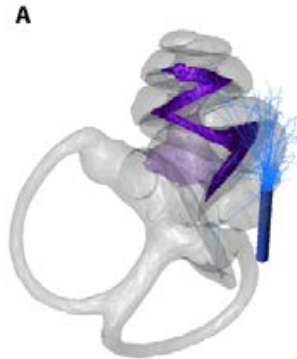

D

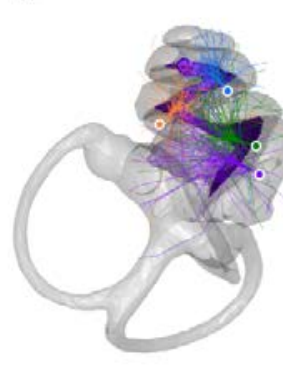

B

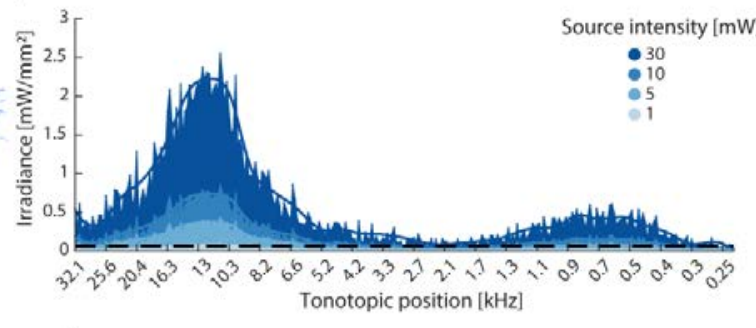

E

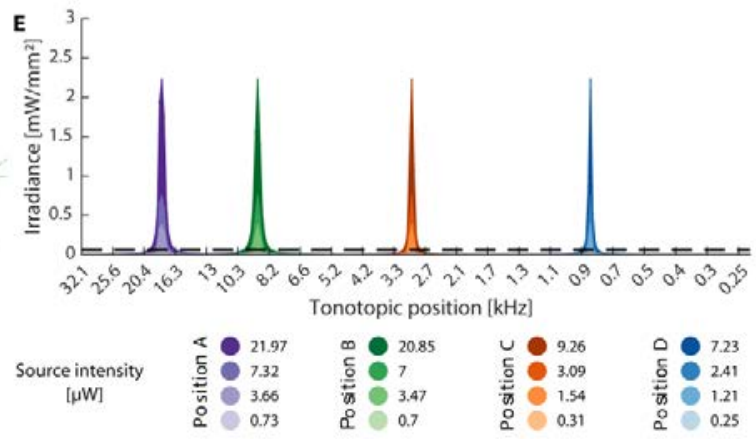

c

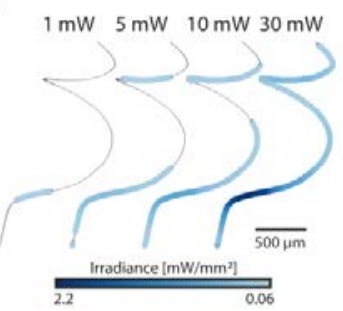

F

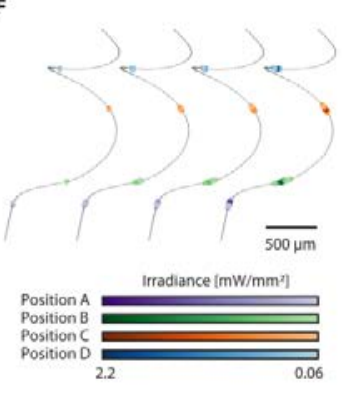

Figure S9. Estimating the spread of excitation using Monte Carlo ray tracing. (A, B, C) Monte Carlo ray tracing simulation from a $200 \mu \mathrm{m}$ optical fiber in a gerbil cochlea $(\lambda=473 \mathrm{~nm})$, comparable to the one used in the in vivo experiments (Thorlabs FT200UMT, 0.39 NA). (A) Morphologically realistic 3D model reconstructed from X-Ray tomography. Purple: Rosenthal's canal, neuronal peripheral processes; light purple: modiolus; Grey: Scala Tympani, Media and Vestibuli as well as semicircular ducts; Blue: Modelled optic fiber. For illustrative purposes, rays with an intensity 50\% above minimum from 7351 rays (out of 3 million used for the simulation) are displayed. (B) Irradiance profile obtained from 300 query points placed along the centerline of the Rosenthal's canal and their corresponding smoothed traces at four different intensities covering the range employed during in vivo experiments. Dashed line represents threshold, considered as the smoothed irradiance value at $10 \mathrm{kHz}$. (C) XY projection of the query points. Color of the query points is displayed as function of the irradiance normalized to the maximal value obtained at $30 \mathrm{~mW}$ for suprathreshold values. Black points represent positions with subthreshold irradiance. (D, E, F) Monte Carlo ray tracing simulation from a Thorlabs FG010LDA optical fiber $(0.1 \mathrm{NA}, \lambda=473 \mathrm{~nm})$ at four different positions. These simulations show narrower spread of light using optimal conditions of position and beam divergence. (D) The origin of the source was placed at the centerline of the scala tympani facing the Rosenthal's canal perpendicularly. The color code is the same as in panel A, with the exception of the light sources: FG010LDA optical fiber output at position A (slate blue), B (green), C (red) and D (blue). For illustrative purposes, rays with an intensity $50 \%$ above minimum from 7351 rays (out of 3 million used for the simulation) are displayed for every position. (E) Irradiance profile obtained as in panel B in four independent simulations (one for each source position). For every source position, the maximum intensity was scaled to the maximum of panel B. The total source intensity needed to achieve comparable irradiances as in panel A is roughly 3 orders of magnitude lower. (F) Merged XY projection of the query points from the four simulations. For each source position, color of the query points is displayed as function of the irradiance normalized to the maximal value obtained at highest source output for suprathreshold values. Black points represent positions with subthreshold irradiance. 
A
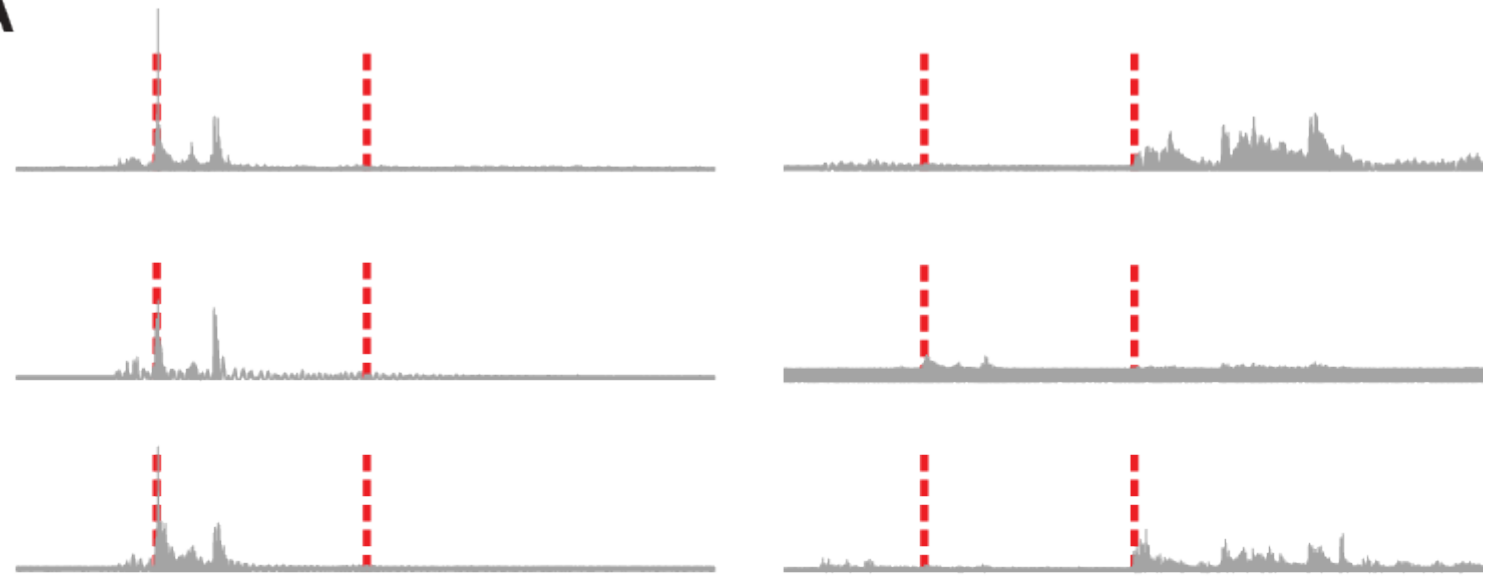

B
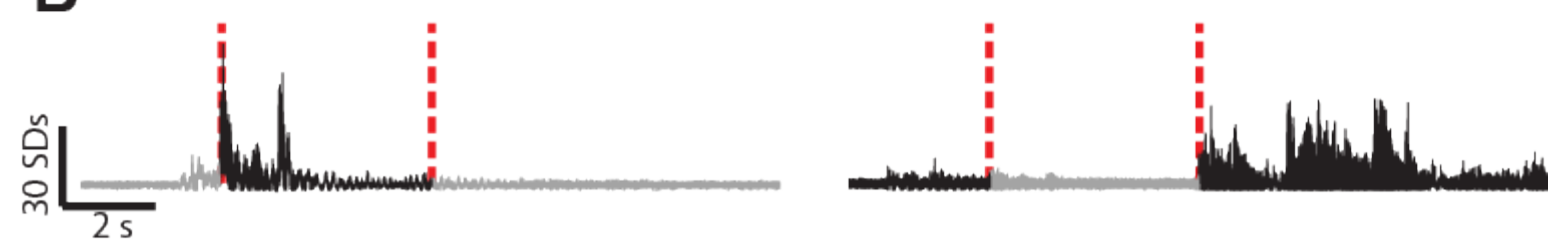

Figure S10. Shuttle box location detection algorithm. (A) Raw data of acceleration sensors (X-, Y-and Z-axis for each side, respectively). (B) Data of acceleration sensors after processing by the algorithm for location-detection ( $y$-axis: standard deviations). Red lines indicate compartment changes detected by the algorithm, black areas mark the compartment in which the animal is currently located. 
Table S1: Number of animals used for each experiment. In addition, 48 AAV-CatCh injected but negative animals have been used for the study, which have not been further analyzed.

\begin{tabular}{|c|c|c|}
\hline Experiment & Number of Animals & Figure \\
\hline Histology of Spiral Ganglion Neurons & $\begin{array}{l}9 \text { CatCh-injected positive (intramodiolar, IM) } \\
4 \text { CatCh-injected negative (IM) } \\
8 \text { Chronos-injected (IM) } \\
3 \text { Chronos-injected (scala tympani, ST) }\end{array}$ & $\begin{array}{l}\text { fig. } 1 \\
\text { fig. S2 } \\
\text { fig. S1 } \\
\text { fig. S1 }\end{array}$ \\
\hline ABR Recordings & $\begin{array}{l}14 \text { CatCh-injected acute } \\
7 \text { CatCh-injected chronic } \\
13 \text { Wildtype acute }\end{array}$ & $\begin{array}{l}\text { fig. 2, 3, fig. S4 } \\
\text { fig. } 2 \text {, fig. } 54 \\
\text { fig. } 2\end{array}$ \\
\hline Auditory Nerve Fiber Recordings & $\begin{array}{l}7 \text { CatCh-injected } \\
6 \text { Wildtype }\end{array}$ & fig. 3 \\
\hline Chronic oCl - ABR Recordings & $\begin{array}{l}7 \text { CatCh-injected } \\
2 \text { PBS-injected }\end{array}$ & $\begin{array}{l}\text { fig. 4, fig. S7 } \\
\text { fig. } 4\end{array}$ \\
\hline Chronic oCl - X-ray Tomography & 2 CatCh-injected & fig. 4, fig. S8 \\
\hline Chronic oCl - Shuttle Box Behavior & $\begin{array}{l}7 \text { CatCh-injected } \\
2 \text { PBS-injected }\end{array}$ & $\begin{array}{l}\text { fig. } 5 \\
\text { fig. } 5\end{array}$ \\
\hline Auditory Cortex Recordings & 12 CatCh-Injected & fig. 6 \\
\hline Optical Stimulation of Deaf Gerbils & 4 CatCh-injected & fig. 7 \\
\hline
\end{tabular}




\title{
Chapter II: Near physiological spectral selectivity of cochlear optogenetics
}

\author{
Alexander Dieter ${ }^{1,2}$, Carlos J. Duque-Afonso ${ }^{1,2,3}$, Vladan Rankovic ${ }^{1,4,5}$, Marcus Jeschke ${ }^{1,4,6,{ }^{*}}$, Tobias \\ Moser $^{1,2,3,4, *}$ \\ ${ }^{1}$ Institute for Auditory Neuroscience and InnerEarLab, University Medical Center Göttingen, 37075 Göttingen, Germany \\ ${ }^{2}$ Göttingen Graduate School for Neurosciences and Molecular Biosciences, University of Göttingen, 37075 Göttingen \\ ${ }^{3}$ Auditory Neuroscience Group, Max Planck Institute for Experimental Medicine, 37075 Göttingen, Germany \\ ${ }^{4}$ Auditory Neuroscience and Optogenetics Laboratory, German Primate Center, 37077 Göttingen \\ ${ }^{5}$ Restorative Cochlear Genomics Group, Auditory Neuroscience and Optogenetics Laboratory, German Primate Center, \\ 37077 Göttingen \\ ${ }^{6}$ Cognitive Hearing in Primates Group, Auditory Neuroscience and Optogenetics Laboratory, German Primate Center, \\ 37077 Göttingen \\ * These authors jointly supervised this work \\ Correspondence: tmoser@gwdg.de
}

Copyright notice:

From: A. Dieter, C.-J. Duque-Afonso, V. Rankovic, M. Jeschke, T. Moser, Near physiological spectral selectivity of cochlear optogenetics. Nat Commun 2019 Apr 29; 10(1)

This is an open access article distributed under the terms of the Creative Commons CC BY license, which permits unrestricted use, distribution, and reproduction in any medium, provided the original work is properly cited.

Under Creative Commons, authors retain copyright in their articles.

DOI: https://doi.org/10.1038/s41467-019-09980-7 


\section{Author contributions:}

A.D., M.J., and T.M. designed the study. M.J. designed hardware and software for multichannel electrophysiological recordings. A.D. performed virus injections, IC recordings and IC histology. C.J.D.-A. performed Monte-Carlo ray trace modeling. V.R. produced the PHP.B-CatCh virus. A.D. performed data analysis under the supervision of M.J. and T.M. A.D., M.J., and T.M. prepared the manuscript.

\section{Detailed author contributions of Alexander Dieter (A.D.):}

\section{Conceptual work:}

- Contribution to experimental design, initially layouted by Prof. Dr. T. Moser and Dr. M. Jeschke

\section{Experimental work:}

- Intramodiolar virus injections in adult Mongolian gerbils

- Cochlear surgery and placement of optical fibers and electrical cochlear implants

- In vivo multi-electrode electrophysiological recordings from the auditory midbrain

- Slice preparation, DAPI-staining and confocal image acquisition of auditory midbrain sections

Data analysis:

- Development of Matlab-Code for spike extraction, analysis, statistics, and data display of electrophysiological recordings

Data presentation:

- Manuscript preparation (first draft and editing) under close supervision of Prof. Dr. T. Moser and Dr. M. Jeschke

- Preparation of figures 1, 2, 3 and 4 displayed in the manuscript and figures $1,2,3,4,5,6,7,9,10,11,12,13$, 14 displayed in the supplementary information 


\begin{abstract}
Cochlear implants (Cls) electrically stimulate spiral ganglion neurons (SGNs) and partially restore hearing to half a million $\mathrm{Cl}$ users. However, wide current spread from intracochlear electrodes limits spatial selectivity (i.e. spectral resolution) of electrical Cls. Optogenetic stimulation might become an alternative, since light can be confined in space, promising artificial sound encoding with increased spectral selectivity. We compare spectral selectivity of optogenetic, electric, and acoustic stimulation by multi-channel recordings in the inferior colliculus (IC) of gerbils. When projecting light onto tonotopically distinct SGNs, we observe corresponding tonotopically ordered IC activity. An activity-based comparison reveals that spectral selectivity of optogenetic stimulation is indistinguishable from acoustic stimulation for modest intensities. Moreover, optogenetic stimulation outperforms bipolar electric stimulation at medium and high intensities and monopolar electric stimulation at all intensities. In conclusion, we demonstrate better spectral selectivity of optogenetic over electric SGN stimulation, suggesting the potential for improved hearing restoration by optical Cls.
\end{abstract}




\section{Introduction}

By stimulating spiral ganglion neurons (SGNs) electrically, cochlear implants (eCls) provide the auditory system of profoundly hearing impaired and deaf with information on the surrounding acoustic scene $e^{1,2}$. eCls are considered the most successful neuroprosthesis and enable open speech comprehension in the majority of approximately 500,000 users. Still, there is an unmet need for improvement: Wide spread of electric current from each electrode contact activates large subsets of SGNs, limiting the number of independent stimulation channels in eCls to less than $\operatorname{ten}^{3-5}$. This major drawback of electric stimulation restricts the amount of spectral information that $\mathrm{Cls}$ can provide to the user, ultimately resulting in limited perception of acoustic signals such as speech, especially in noisy environments ${ }^{5,6}$.

Optical stimulation of SGNs represents a novel approach to overcome this limitation of eCls: Light can be better confined in space and, hence, optical cochlear implants (oCls) could activate SGNs along the tonotopic axis of the cochlea with higher spatial selectivity. This promises improved spectral resolution of artificial sound coding and consequently an increased number of independent stimulation channels ${ }^{7-10}$. Studies of cochlear activation using infrared stimulation have indicated that spatial (and thus spectral) spread of SGN excitation in the cochlea is small for optical stimulation, comparable to pure tone acoustic stimulation ${ }^{7}$, while monopolar electrical stimulation led to spectrally broader SGN activation than infrared stimulation in a different study $^{11}$. However, the energy requirement per pulse is very high for infrared stimulation (16-160 $\mu \mathrm{J}^{12}$ ), the exact mechanism of neural activation is still under debate and activation of the auditory pathway could not be verified in several studies on animal models of sensorineural hearing loss ${ }^{13-}$ 15 .

In contrast, optogenetic stimulation of SGNs expressing Channelrhodopsins (ChRs ${ }^{16,17}$ ) enables neural excitation by a well understood mechanism at lower light intensities. Indeed, stimulation of the auditory system using fiber-based $\mathrm{oCl}$ has greatly advanced in the past years: a proof of principle study employing cochlear optogenetics in transgenic mice demonstrated optical activation of the auditory pathway up to the inferior colliculus (IC), where current source density analysis indicated a smaller spread of SGN excitation for optical than for monopolar electrical stimulation $^{8}$. Subsequent studies on mice in which SGNs were virally transduced during the first postnatal week using the fast-gating ChRs Chronos and f-Chrimson demonstrated high temporal fidelity of neural control up to several hundred Hertz by recording optogenetically driven auditory brainstem responses (OABRs) and spiking activity of individual SGNs in hearing and deaf 
animals ${ }^{18,19}$. Finally, viral transduction of SGNs in adult Mongolian gerbils was recently established and optogenetic stimulation of the auditory nerve was studied by recordings of OABRs and individual $\mathrm{SGNs}^{20}$. Furthermore, stimulus perception upon optogenetic stimulation of the auditory nerve was demonstrated by single unit recordings from primary auditory cortex and behavioral experiments, the latter also involving deafened animals.

However, despite the recent progress, a precise estimation of the spectral selectivity of optogenetic SGN stimulation and a rigorous comparison to acoustic and electrical stimulation is still lacking. Here, we perform multi-channel recordings of neuronal clusters (multi-unit activity) in the tonotopically organized central nucleus of the inferior colliculus (ICC) in Mongolian gerbils while stimulating SGNs optogenetically, electrically or acoustically. We demonstrate spatially selective optical activation of the auditory system in a tonotopic manner with a spectrally more confined SGN excitation than the one found upon monopolar and bipolar electrical stimulation. This indicates increased spectral resolution of artificial sound encoding when using optogenetic instead of electrical stimulation - and thus suggests that oCls might overcome the major bottleneck of eCls. 


\section{Results}

Mapping acoustic response

properties in the central nucleus of the inferior colliculus (ICC): To characterize spectral response properties of the auditory system to optogenetic stimulation of SGNs we performed electrophysiological recordings of multi-unit activity in the ICC in isoflurane-anesthetized gerbils. The ICC was chosen because of its well-defined tonotopic organization, enabling estimation of the cochlear spread of excitation of acoustic electrical optogenetic stimulation (oCl). We used multiple laser-coupled optical fibers placed at three different positions along the tonotopic axis of the cochlea (Fig. $1 \mathrm{~A}-\mathrm{C})$ and recorded activity in the ICC using linear 32-channel silicon probes.

After placing the silicon probe, frequency response areas were constructed for each recording site
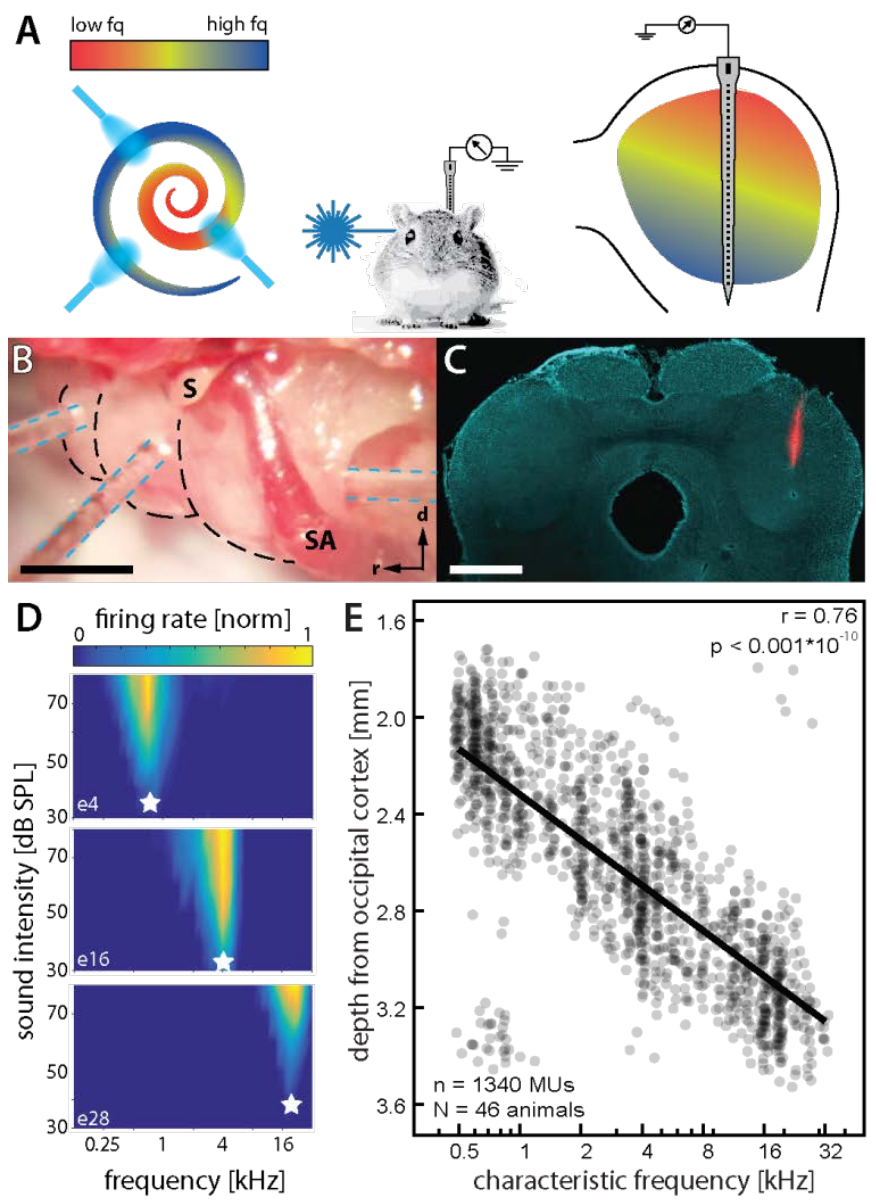

Figure 1: Experimental layout and acoustic response properties. (A) Experimental design. (B) Optical fibers (blue dashed lines) inserted via cochleostomies at the apical and mid-turn of the cochlea (black dashed lines) as well as in the round window (from left to right). S: stapes, SA: stapedial artery. Scale bar: $1 \mathrm{~mm}$. (C) Dil-stained electrode track (red) in a DAPI-stained (cyan) coronal section of the inferior colliculus. Scale bar: $1 \mathrm{~mm}$. (D) frequency response areas and characteristic frequencies (CFs; white stars) recorded at electrode 4, 16 and 24 (e4, 16,28 ) in one animal. (E) CFs as a function of recording depth. Solid line: linear fit of all CFs, according to Pearson's correlation coefficient. Data is pooled from all animals (virus-injected as well as non-injected). Source data of $(E)$ is provided as a source data file. using acoustic stimulation. We then derived the characteristic frequencies (CFs) (Fig. 1D, E) and calculated tonotopic slopes for each animal by linearly fitting the CFs as a function of recording depth (Fig. 1E, Supplementary Figure 1). The median tonotopic slope amounted to 4.58 octaves $/ \mathrm{mm}$ ( \pm 0.69 median average deviation, $n=46$; Supplementary Figure 1, inset) and did not differ between animals that underwent cochlear surgeries and naïve animals $(4.61 \pm 0.70$ octaves $/ \mathrm{mm}, n=38$ vs. $4.58 \pm 0.60$ octaves $/ \mathrm{mm}$, $n=8$; two-sample t-test: $p=0.69)$. However, in animals that underwent cochlear surgeries, 
thresholds of acoustically driven multi-units in the ICC increased by $17.8 \mathrm{~dB}$ on average $(30.3 \pm 13.4 \mathrm{~dB}$ SPL standard deviation $(n=$ 246) in naïve animals, 48.1 $\pm 11.6 \mathrm{~dB}$ SPL $(n=208)$ in animals that underwent surgery, $p<0.001 * 10^{-35}$, two-sample t-test;

Supplementary Figure 2).

Artificial stimulation of spiral ganglion neurons: To prove stimulation of SGNs via $\mathrm{oCl}$ or $\mathrm{eCl}$ and determine time windows for potential responses, peri-stimulus time histograms (PSTHs) were constructed in response to the strongest optical and electrical stimuli (Supplementary Figure 3). Potential responses to various stimulus intensities were then evaluated during these time windows as well as during the presentation of tone bursts for acoustical stimulation. The majority of acoustically driven multi-units (98.1\%) and all optically and
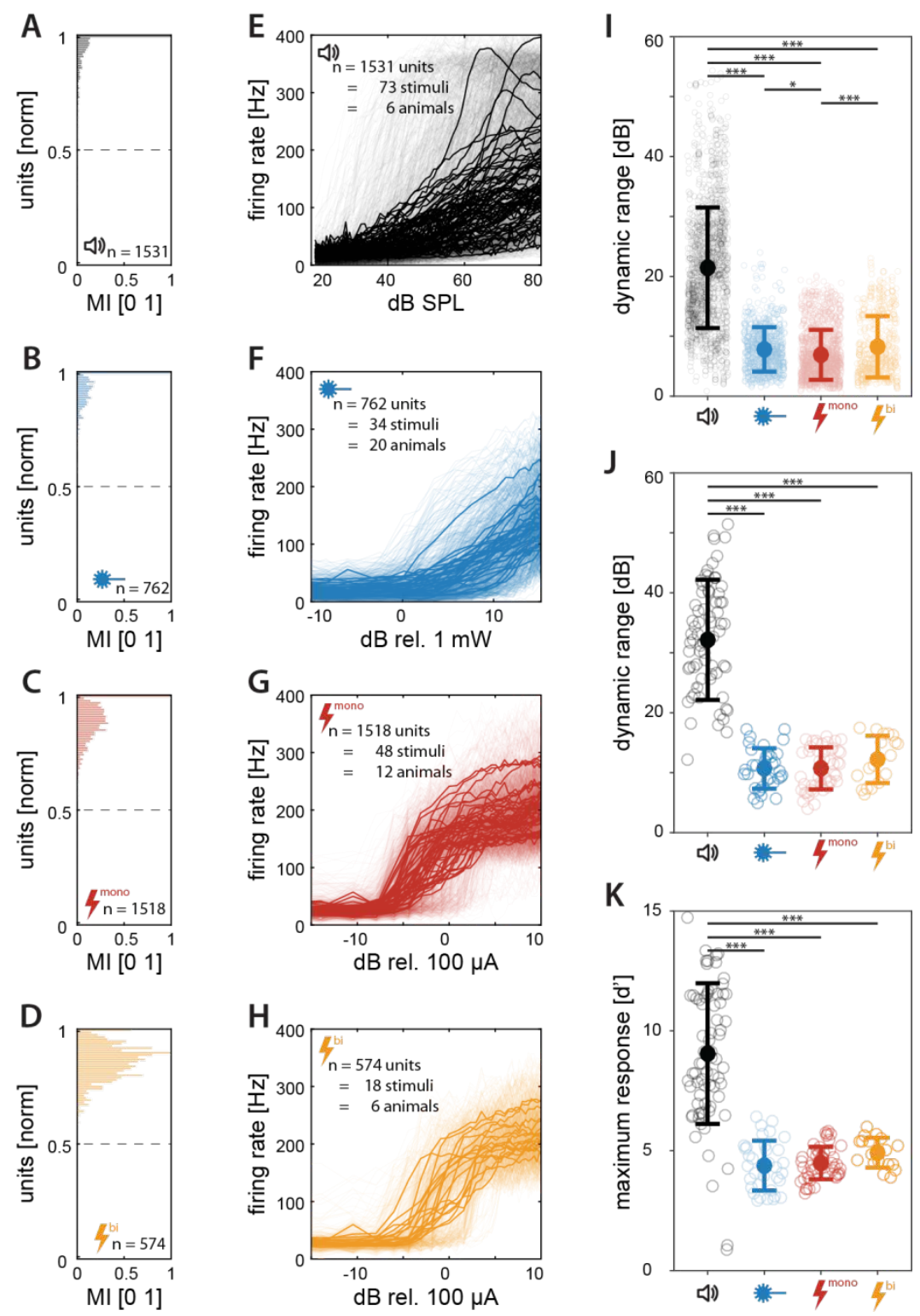

Figure 2: Acoustic, optogenetic and electric activation of the auditory system. (AD) Monotonicity indices (MI) for acoustic stimulation in non-injected animals (A), optogenetic stimulation in virus-injected animals (B), monopolar (C) and bipolar electric stimulation in non-injected animals (D). Dashed lines mark a monotonicity index of 0.5 , above which units are considered as monotonic. (E-H) Firing rates of multi-units as a function of stimulus intensity (thin, transparent lines) and average per animal and stimulus (solid lines) for acoustic (E), optogenetic (F), monopolar (G) and bipolar (H) electric stimulation. (I) Distribution of dynamic ranges (DRs) per multi-unit in $d B(S P L), d B(m W)$ and $d B(\mu A)$. (J) DRs of averaged multi-units per animal and stimulus, units as in (E). (K) Maximum strength of response that could be evoked by any stimulus modality. Data in $(\mathrm{I}),(\mathrm{J})$ and $(\mathrm{K})$ is displayed as mean \pm s.d.. Stars indicate statistical significance (one star: $p<0.05$, two stars: $p<0.01$, three stars: $p<0.001$ ), based on one-way ANOVA and post-hoc pairwise comparison. Only significant differences are indicated. Source data of all panels is provided as a source data file. 
electrically driven units showed a monotonic firing pattern, i.e. a rise in stimulus intensity led to a rise in firing rate (monotonicity index $>0.5$; Fig. 2A-H). This observation allowed for estimation of the dynamic range (DR) based on stimulus-response functions both per individual multi-unit and per animal with a similar procedure. The DR was calculated as the range of stimulus intensities that led to a monotonic increase in firing rates in the range from $10 \%$ above baseline activity (average response to the three lowest stimulus intensities) to $10 \%$ below maximum response (averaged response to the three highest stimulus intensities). We note that saturation was not achieved for most of the optogenetically driven multi-unit responses (Fig. 2F), such that the apparent DR underestimates the true DR for optogenetic stimulation.

The average apparent DR of individual multi-units in response to optical stimulation amounted to $7.8 \mathrm{~dB}$ ( $\mathrm{mW}$; \pm 3.7 s.d.; $n=762$ ), which exceeded the DR in response to monopolar electrical stimulation (6.9 $\pm 4.2 \mathrm{~dB}[\mu \mathrm{A}], n=1515, p<0.05$; one-way ANOVA and post-hoc pairwise comparison) and was comparable to the DR in response to bipolar electrical stimulation $(8.3 \pm 5.1$ $\mathrm{dB}[\mu \mathrm{A}], n=572 ; p=0.65$; Fig. $2 \mathrm{H}$ ). The $\mathrm{DR}$ in response to acoustic stimulation exceeded all modalities of artificial cochlear stimulation and amounted to $21.4 \pm 10.1 \mathrm{~dB}$ (SPL) ( $n=1531 ; p<$ 0.001). The grand average DR (derived from the mean of the averaged multi-units per animal) was similar between responses to optical (10.7 $\pm 3.4 \mathrm{~dB}$ standard deviation, $n=34)$, monopolar (10.7 \pm $3.5 \mathrm{~dB}, n=48 ; p=0.99)$ and bipolar electrical stimulation (12.2 $\pm 3.9 \mathrm{~dB}, n=18 ; p=0.88)$, while the DR of acoustic stimulation amounted to $32.3 \pm 10 \mathrm{~dB}$ ( $n=73$; Fig. $2 \mathrm{~J}$; one-way ANOVA and post-hoc pairwise comparison). Comparison of the maximal strength of cochlear excitation measured in $d^{\prime}$ values based on evoked firing rates - revealed that optical stimulation could drive neurons in the ICC as effectively as electrical stimulation (max. response: $4.4 \pm 1.0 \mathrm{~d}$ ' values mean and SD, $n=34$; compared to $4.5 \pm 0.7 \mathrm{~d}^{\prime}$ values for monopolar stimulation $(n=48 ; p=0.99$ ) and $4.9 \pm 0.6 d^{\prime}$ values for bipolar stimulation $(n=18 ; p=0.79)$, respectively), whereas acoustic stimulation of non-injected animals yielded stronger activation $(9.0 \pm 2.9$ d'-values; one-way ANOVA and post-hoc pairwise comparison; Fig. $2 \mathrm{~K}$ ). We note that no responses to optical stimulation of SGNs were observed in non-injected control animals, excluding excitation unrelated to the optogenetic mechanism such as opto-acoustic or -thermal effects and thus proving the specificity of optogenetic SGN stimulation (Supplementary Figure 4).

Spectral spread of fiber-based cochlear optogenetics: To thoroughly characterize the spread of excitation upon optical stimulation, we used a multi-site approach to project light onto SGNs at three distinct tonotopic positions. For this purpose, optical fibers were inserted into the cochlea i) 
via the round window (high-frequency base) and via cochleostomies in the ii) middle (midfrequency) and iii) apical cochlear turn (low-frequency), (Fig. 1B).

For comparing the optogenetic spread of excitation to that of acoustic and electrical stimulation, we also performed recordings while stimulating naive animals that did not undergo any surgical manipulation of the cochlea using pure tones and stimulating non-injected animals with a 4channel $\mathrm{eCl}$ using monopolar electrical stimulation, where the return electrode was placed outside of the bulla tympanica and bipolar electrical stimulation where the electrode next to the stimulation electrode (in basal direction) served as the return electrode. Neuronal activity was recorded in response to a given modality of varying stimulation intensity (pure tones, optically via one of the fibers, or electrical stimulation via one of the four eCl electrodes Fig. 3).
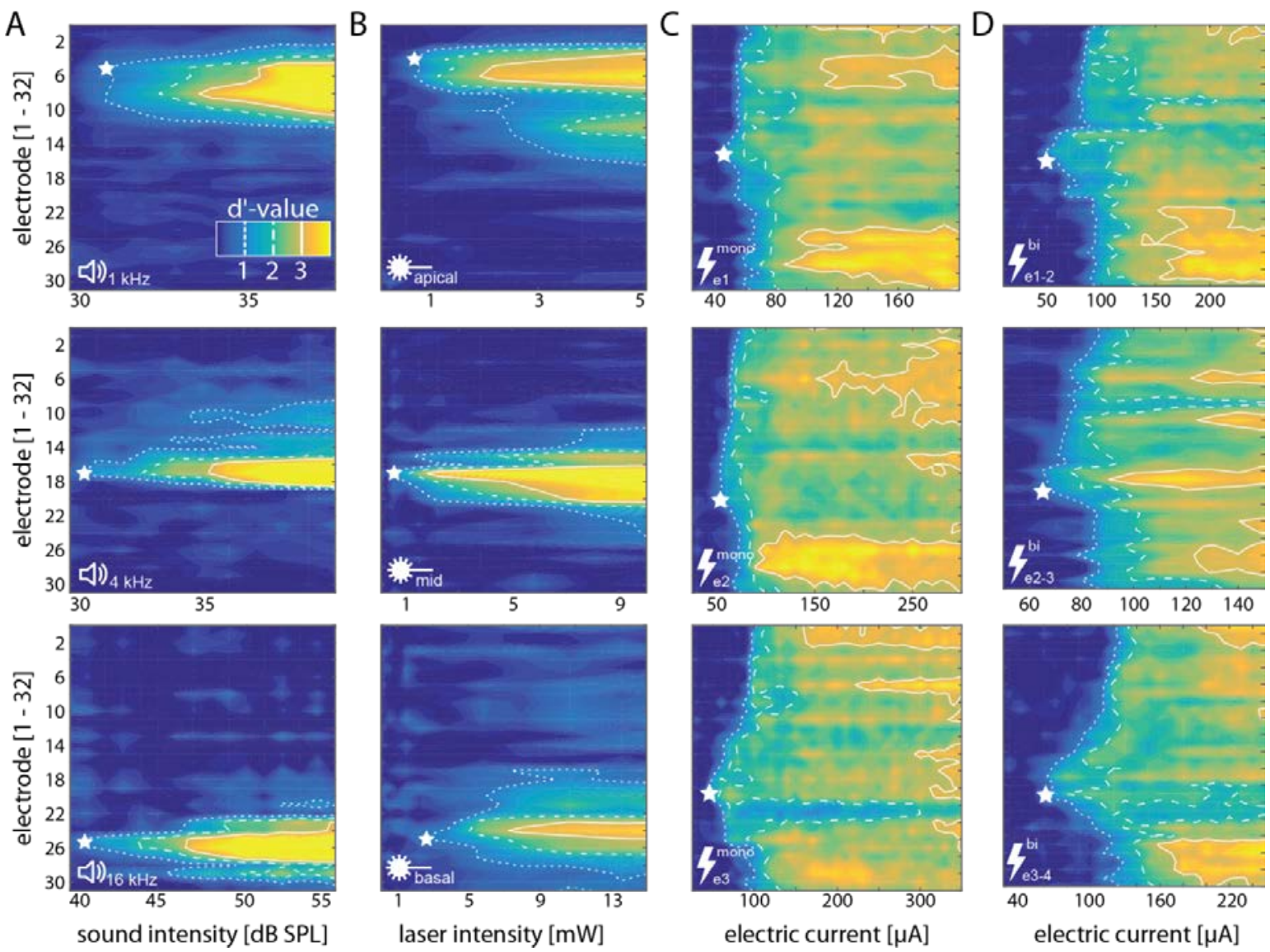

Figure 3: Exemplary spatial tuning curves (STC) for different stimulus modalities. (A) STCs in response to pure tones of different frequencies in naive animals. (B) STCs in response to laser pulses delivered via optical fibers placed at different positions in the cochlea of virus-injected animals. (C) STCS in response to electric current delivered via different electrodes (e1, e2 and e3) of an electrical cochlear implant in non-injected animals in the monopolar configuration. (D) STCs in response to electric current delivered via the same electrodes in bipolar configuration. White stars indicate the best electrode (BE) of each STC. The color scale in (A) applies to all panels displayed in this figure. 
To characterize the change in multi-unit firing rates to changing stimulation levels across different modalities we employed a method based on signal detection theory ${ }^{21}$. A cumulative $d^{\prime}$-value based on multi-unit spike rates in response to increasing stimulation intensities was calculated, starting in the absence of stimulation (i.e. zero intensity). Cumulative $d^{\prime}$-values for increasing stimulus intensities were then sorted into a response matrix according to the electrode they were recorded from and iso- $d^{\prime}$-contour-lines were drawn at integer $d^{\prime}$-values in order to construct spatial tuning curves (STC; Fig. 3 and 4). As in previous studies on eCl, a d'value of 1 was defined as the threshold and the recording electrode with the lowest threshold was defined as the best electrode (BE; 16, 25). Average thresholds amounted to $2.67 \mathrm{~mW}$ for optical, and $45.8 / 52.8 \mu \mathrm{A}$ for mono-/bipolar electrical stimulation (Supplementary figure 5).

In order to quantify the cochlear spread of excitation, we measured the distance between all active electrodes $\left(d^{\prime}>1\right)$ at the stimulus intensity at which the BE reached a given $d^{\prime}$ value (i.e. $1.5,2,2.5$ or 3 ; Fig 4A). In some cases, more than one peak (defined as electrodes below threshold separating electrodes above threshold) has been observed for each stimulus modality (acoustic: 33/304; optogenetic: 27/101; monopolar electric: 25/192; bipolar electric: 7/72). In these cases, the dorsal- and ventral-most electrodes with a significant response $\left(d^{\prime}>1\right)$ have been considered as the boundaries of the STC to avoid underestimation of the spread of excitation (Supplementary Figure 6). Using the tonotopic slopes calculated above, spatial spread in each animal (measured in distance between the electrodes) could then be translated into spectral spread of cochlear excitation (measured in octaves). Measuring at fixed significance of response strengths - i.e. at identical levels of activation - rather than at fixed stimulus intensities, the estimation of the spread of excitation becomes independent of the stimulus' nature and makes neural activation by different modalities more comparable. 

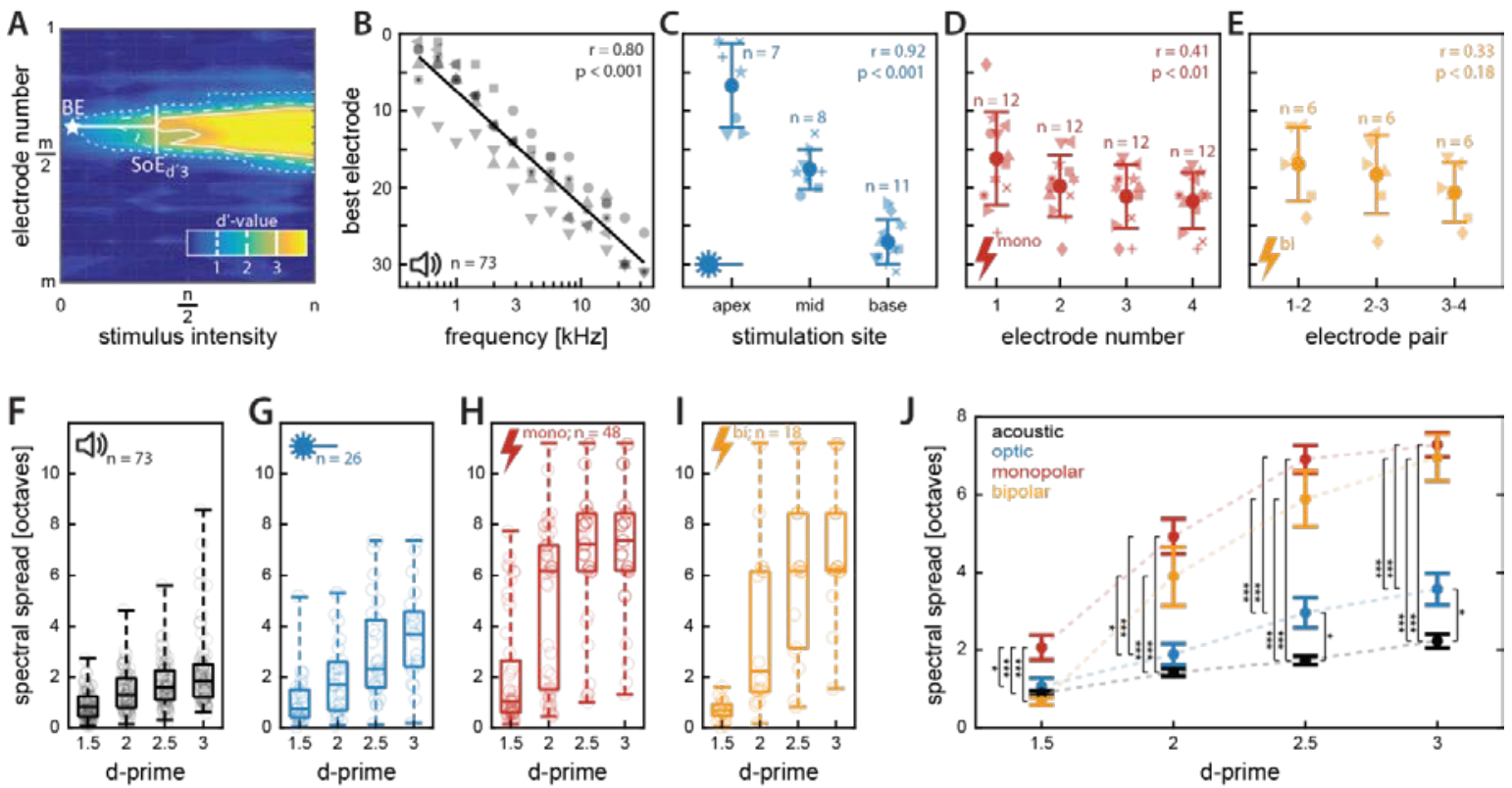

Figure 4: Quantification of spatial tuning curves (STCS). (A) Quantification of STCs: STC in response to a $2 \mathrm{kHz}$ pure tone in a non-injected gerbil. Cumulative d-prime values are color-coded in a matrix sorted according to electrode number (ordinate) and stimulus intensity (abscissa). The electrode with the lowest threshold $\left(d^{\prime}=1\right)$ is defined as the best electrode ( $\mathrm{BE}$, white star) and the spread of excitation $(\mathrm{SOE})$ is measured at different activation strengths (exemplary shown for a d' of 3 at the BE). (B) BEs as a function of stimulation frequency recorded from non-injected gerbils. Solid line: linear fit of tonotopic slope. Different symbols mark different animals. (C) BEs as a function of optical stimulation site in virus-injected animals, including mean and standard deviation for each stimulation site. Symbols mark different animals. (D) BEs as a function of stimulation electrode for monopolar electrical stimulation in non-injected animals, including mean and standard deviation for each electrode. (E) BEs as a function of stimulating electrode pair for bipolar electrical stimulation in non-injected animals, including mean and standard deviation for each electrode pair. Pearson's correlation coefficient $r$ and the corresponding $p$-values were calculated for panel B-E. (F-I) Spectral spread has been quantified at different $d^{\prime}$-values for acoustic $(F)$, optogenetic $(G)$, monopolar $(H)$ and bipolar electric stimulation (I). Box plots indicate minimum (lower) and maximum (upper) whisker, median (center line) as well as 25- and 75-percentiles. (J) Mean and SEM for the spread of excitation upon acoustic, optogenetic, monopolar and bipolar electric stimulation. Stars indicate statistical significance (one star: $p<0.05$, two stars: $p<0.01$, three stars: $p<0.001$ ), according to repeated-measures ANOVA and post-hoc pairwise comparison tests. Only significant differences have been indicated. Source data of panels B-J is provided as a source data file.

Plotting the focus of activation (i.e. BE) as a function of stimulation frequency visualizes the natural tonotopic axis of the ICC with an average tonotopic slope of 4.48 octaves/mm (Pearson's correlation coefficient $r=0.80, p<0.001$; Fig. 3A, also see Fig. 1 and Fig. 4B). Similarly, neuronal responses could be shifted systematically from the dorsal to the ventral ICC when stimulating optically at the apex, mid or base of the cochlea in AAV-injected animals $(p<0.001$; Fig. 3B, Fig. 4C, Supplementary Figure 7A). A systematic shift of activation was also apparent for eCl stimulation via different electrodes upon monopolar electrical stimulation $(p<0.001$; Fig. 3C, also see Fig. 4D) but was not significant when stimulating in bipolar configuration $(p=0.18)$. 
Nonetheless, when probing tonotopy of stimulation by correlating stimulating electrode (pairs) with the CF recorded at the best electrode it was significant for both mono- and bipolar electrical stimulation ( $r=0.31, p<0.05$ and $r=0.6, p<0.01$, respectively; Supplementary Figure 7B-C). Note that the layout of the $\mathrm{eCl}$ might have contributed to the less pronounced tonotopic activation, as the 4 electrodes only covered $1.8 \mathrm{~mm}$ in the case of monopolar and $1.2 \mathrm{~mm}$ in the case of bipolar electrical stimulation and thus were not distributed along the whole cochlea, whereas optical fibers have been placed in a way to cover large parts of the cochlear spiral.

In order to better understand the spread of light upon optical stimulation and the variance introduced by fiber-positioning, we modelled $\sim 3,000,000$ optical rays in a Monte-Carlo simulation where optical fibers were placed at the corresponding positions within a model of the gerbil cochlea reconstructed from $\mathrm{x}$-ray phase-contrast tomography (Supplementary Figure 8). Peak illumination of the spiral ganglion in Rosenthal's canal was observed at tonotopic places corresponding to $1.01,6.9$ and $22.89 \mathrm{kHz}$, spanning a total of 4.43 octaves. Varying the angle of light projection shifted the peak of illumination at the spiral ganglion, which (just as precise axial positioning of the fiber aperture) contributes to the variance of the experimentally observed BES and CFs upon optical stimulation.

Spread of excitation at increasing $d^{\prime}$ values grew for all stimulus modalities (Fig. 4F-I). At a d' of 1.5 and 2, the spread of excitation of optogenetic stimulation was indistinguishable from that upon pure tones suggesting that near physiological frequency resolution might be achievable in future $\mathrm{oCl}$ for modest stimulation strength (Repeated-measures ANOVA and post-hoc pairwise comparison tests; Fig 4J). At higher activation levels ( $d^{\prime}$ of 2.5 and 3 ) the spread of excitation was significantly higher for optogenetic stimulation $(p<0.05)$ as compared to acoustic stimulation. However, optogenetic stimulation outperformed monopolar eCl stimulation at all activation strengths ( $p<0.05$ at a $d^{\prime}$ of $1.5 ; p<0.001$ at all other activation strengths). Furthermore, optogenetic stimulation performed significantly better than bipolar electrical stimulation at medium ( $d^{\prime}$ of $2 ; p<0.05$ ) and high activation levels ( $d^{\prime}$ of $2.5 / 3 ; p<0.001$ ), while no difference was found at low activation levels ( $d^{\prime}$ of $1.5 ; p=0.77$ ). These findings were identical when the spectral spread of excitation was not normalized by the tonotopic axis of each animal, i.e. when measuring the spread of excitation in terms of spatial activation in the ICC (Supplementary Figure 9). For optical stimulation via the round window we also compared the fiber orientation projecting light onto SGNs of $\mathrm{i}$ ) the high-frequency base (used in the present comparison (Figs. 3 and 4) and ii) the orientation pointing towards the cochlear apex and thus projecting light along 
the modiolar axis (used in our previous study ${ }^{20}$ ), which led to a rather broad activation of the spiral ganglion (Supplementary Figure 10). Thus, the precise projection of light to the spiral ganglion is of critical importance to achieve high spectral resolution by oCl stimulation.

These findings suggest that optical excitation of SGNs happens in a more spatially confined manner than electrical stimulation and hence can achieve better spectral resolution, provided appropriate projection of the light. 


\section{Discussion}

In this study, we scrutinized the spectral spread of excitation for optogenetic stimulation of SGNs and compared it to physiological hearing and hearing with eCl employing monopolar stimulation, used in the majority of clinical $\mathrm{eCls}^{23}$, as well as bipolar stimulation. We could demonstrate a major advantage of optogenetic over monopolar and bipolar electrical stimulation for this highly relevant parameter of artificial sound encoding. In fact, the study indicates that optogenetic stimulation can achieve near physiological frequency selectivity at low to modest levels of activation. We attribute this primarily to the spatial confinement of optical stimulation even with the rather crude oCl implementation in our current study.

A prerequisite for quantifying cochlear spread of excitation based on ICC measurements is reliable and reproducible positioning of electrode arrays along the tonotopic axis of the ICC. Hence, we placed the array under guidance of neuronal responses to acoustic stimuli. The expected correlation of electrode CF and depth in the ICC was observed for each animal in this study. The median tonotopic slope amounted to 4.58 octaves $/ \mathrm{mm}$ based on CFs and to 4.48 octaves $/ \mathrm{mm}$ based on the BEs, which compares well to literature ( 4.08 and 4.37 octaves $/ \mathrm{mm}$, respectively ${ }^{24,25}$ ). A few units in ventral ICC regions were found to be responsive to low frequency tones, indicating that recording sites for these units were likely outside the ICC ${ }^{24}$. However, since these were only 30 out of 1340 units, $97.76 \%$ of multi-units recorded in this study can be considered to primarily originate from the ICC. As the external cortex, covering the ICC dorsally and laterally, is thin (less than $150 \mu \mathrm{m}^{26,27}$ ) we have not attempted to separate the expectedly few cortical neurons contributing to the data set.

Optogenetic stimulation of SGNs at different positions of the cochlear spiral evoked neural activity in tonotopically corresponding ICC regions: apical stimulation evoked activity in the dorsal, low frequency regions of the ICC while baso-cochlear stimulation excited ventral, high frequency parts. Tonotopic ICC activation was less obvious when using electrical stimulation. We note that appropriate positioning of the $\mathrm{oCl} / \mathrm{eCl}$ within the cochlea is critical for its tonotopic activation: Optical fibers were placed in 3 spatially distinct positions to cover a large tonotopic range (see fig. $1 B, 4 C$, Supplementary Figure 5A; i.e. regions coding centred around 0.6, 3.6 and $13.3 \mathrm{kHz}$ from apical, mid and basal stimulation, spanning a total of 4.5 octaves, i.e. $56 \%$ of the gerbils hearing range). This was confirmed by modelling optical rays from the fiber aperture placed in a reconstructed gerbil cochlea (Supplementary Figure 8). In contrast, electrodes of the eCl spanned 
$1.8 \mathrm{~mm}$ (monopolar) and $1.2 \mathrm{~mm}$ (bipolar) of the scala tympani, covering only $16.4 \%$ and $10.9 \%$ (i.e. 0.29 and $0.19-$ fold of optical stimulation) of the cochlear length ${ }^{28}$. Experimentally, average best electrode IDs in response to optical stimulation upon apical and basal stimulation were 6.7 and 27.9, whereas in response to electrical stimulation the electrode IDs for apical- and basalmost mono- or bipolar stimulation were 16.5 / 21.8 or $17 / 20.7$ (i.e. 0.25 -fold of 0.17 -fold optical stimulation, respectively). These ICC estimates agree well with cochlear tonotopic ranges estimated above for optical and electrical stimulation, suggesting that tonotopic activation was achieved in a comparable manner.

The spread of cochlear excitation determines the spectral resolution of artificial sound coding which is limited with current eCls. A previous study on cats (where the spread of excitation was quantified by measuring the width of ICC activation $6 \mathrm{~dB}$ above threshold) reported 2.55 and 4.94 octaves of neural activation for bi- and monopolar stimulation, compared to 0.6 octaves for pure tones $^{21}$. Electrical stimulation via an intra-neural electrode array (penetrating the auditory nerve) outperformed eCls conventionally placed in the scala tympani, activating only 1.4 octaves in the same study. When analyzing our data as done in this study ( $6 \mathrm{~dB}$ above threshold, corresponding to a mean/SD d' of $3.02 \pm 0.64$ ), the spread of excitation in our study amounted to $1.81 \pm 0.7$ (SD; $n=71), 2.91 \pm 1.6(n=20), 6.36 \pm 2.3(n=44)$ and $6.07 \pm 2.6(n=16)$ octaves for acoustic, optogenetic, monopolar and bipolar electric stimulation, respectively (Supplementary Figure 11).

Since the species used in these studies differ both in physiology and anatomy, we suggest normalizing the spread of excitation to the corresponding spread of excitation upon pure tone stimulation employed in each study to facilitate a better comparison. By doing so, the spread of excitation with the penetrating array of the above-mentioned study corresponds to 2.33 -fold of the acoustic one, while bi- and monopolar stimulation of $\mathrm{eCl}$ in the scala tympani amounted to 4.16 and 8.23 -fold spread. The spread of excitation evoked by optical stimuli in our study amounted to 1.61-fold, while the ones of monopolar and bipolar electrical stimulation amounted to 3.51 and 3.35-fold spread, respectively. Another study, performed in guinea pigs reported 3.9fold and 1.8-fold spread of excitation upon mono- and bipolar electrical stimulation, respectively (measured $6 \mathrm{~dB}$ above threshold; compared to acoustic stimulation $20 \mathrm{~dB}$ above threshold) ${ }^{22}$. Comparing our data in this way, we found 0.74 -fold spread of excitation upon optogenetic and 1.61-/1.53-fold excitation upon mono- and bipolar electrical stimulation, respectively (Supplementary Figure 11). 
Not finding the previously reported advantage of bipolar electrical stimulation over monopolar stimulation in our study is most likely explained by the fact that the majority of STCs upon electrical stimulation have been underestimated. The boundaries of recorded STCs exceeded the limits of the electrode array in many cases (i.e. neural activity was evoked at all recording sites). Since the supposedly more focused bipolar stimulation already evokes activity in most parts of the cochlea (and thus the ICC), less selective stimulation in the monopolar configuration could not be demonstrated. Therefore, we found bipolar stimulation to be more selective than monopolar stimulation only for weak stimuli ( $d$ ' of 1.5 ). We attribute this finding primarily to the model system we used: since the gerbil cochlea is roughly 2.5 times smaller than the cat cochlea and $\sim 1.6$ time smaller than the guinea pig cochlea, a physically similar current spread from the $\mathrm{eCl}$ electrode will lead to activation of a larger SGN population in the gerbil than in the $\operatorname{cat}^{21,28-31}$. Thus, the advantage of bipolar, and importantly, even more so of optical stimulation, over monopolar electrical stimulation - especially at high activation strengths - is expected to be greater in species with larger cochleae, e.g. cats or even humans (even in the guinea pig, $60 \%$ and 93\% of the STCs upon mono- and bipolar electrical stimulation exceeded the boundaries of the recording electrode array and thus might have been underestimated ${ }^{22}$ ). Despite the limited size of the gerbil cochlea, however, we could still demonstrate spatially selective SGN activation upon optogenetic stimulation, approaching the frequency resolution of natural acoustic stimulation.

Taken together, these results suggest that optical stimulation of cochlear neurons is indeed more confined in space than electrical stimulation, despite the non-optimal projection of light from a fiber aperture placed at an opening of the cochlear capsule (Supplementary Figure 8). We suppose that the spread of excitation upon optogenetic SGN stimulation might be even lower when placing light sources into the scala tympani (i.e. closer to the target tissue), using emitters with a lower numerical aperture or by combining light emitters with focusing lenses. Indeed, ray modeling studies indicate narrow tonotopic ranges of activation under these conditions (Supplementary Figure 8C/D).

The output dynamic range of individual multi-units in response to sound (21.4 dB) and electrical stimulation (6.9 dB and $8.3 \mathrm{~dB}$ for mono- and bipolar stimulation) are in good agreement with literature values: an average DR of $20 \mathrm{~dB}$ per single unit in response to sound has been reported for the gerbil IC and DRs between 6.7-7.6 dB have been reported upon stimulation with different kinds of $\mathrm{eCls}$ in the rat $\mathrm{IC}^{31-33}$. DR comparison of optogenetic to electrical stimulation is 
confounded by the fact that we did not find saturation for most multi-units at the light intensities amenable to our set-up (Fig. 2E, Supplementary Figure 12).

The resulting apparent $\mathrm{DR}$ of $7.8 \mathrm{~dB}(\mathrm{~mW})$ was only slightly larger than the $\mathrm{DR}$ in response to monopolar, and comparable to bipolar electrical stimulation. We note that our DR estimation for optical stimulation refers to $\mathrm{dB}(\mathrm{mW})$, i.e. $\mathrm{DR}=10 \times \log 10 \frac{\text { power }(90 \%)}{\text { power }(10 \%)}$, while that of electrical stimulation was based on current amplitudes, i.e. DR $=20 \times \log 10 \frac{\text { amplitude }(90 \%)}{\text { amplitude }(10 \%)}$. Future studies involving behavioural analysis should provide psychophysical estimates of DR as well as of the intensity discrimination. Furthermore, optimized opsins conferring increased light sensitivity might lower the threshold of SGN activation and thus increase the DR at the lower end. Biosafety studies of long term exposure to light illumination need to be done in order to determine safe margins for light stimulation that might limit the DR at the upper end.

An obvious limitation of our study is the use of multiple laser-coupled optical fibers placed near the cochlear lateral wall at variable distance to SGNs (Supplementary Figure 8). This approach is not feasible for clinical translation. Due to the spatial flexibility of placing optical fibers at arbitrary cochlear positions, it was possible to access the tonotopic axis of the spiral ganglion throughout the whole cochlea, from the round window up to the apex. Even though the technical feasibility of oCls, e.g. in the form of miniaturized LEDs on a flexible substrate ${ }^{34}$, has been demonstrated, chronic translational experiments requiring stable multi-channel oCls have not yet been reported to our knowledge. A second - apparent - limitation of the current study is that artificial stimulation of SGNs was done in the presence of inner hair cells, i.e. in normal hearing animals. We can assume that the presented results would not differ in deaf animals since we have shown in a previous study that opsin expression is absent in inner hair cells due to the choice of the promotor (human synapsin) and furthermore optogenetic excitation of SGNs is feasible in a model of sensorineural hearing loss ${ }^{20}$. Also, we showed that there was no effect of optical stimulation in non-injected animals, ruling out the contribution of non-optogenetic neural excitation by light. 


\section{Methods}

Animals: Data was recorded from 46 adult (> 8 weeks of age) Mongolian gerbils (Meriones unguiculatus) of either sex. For each surgery, gerbils were anesthetized with Isoflurane (4\% at $1 \mathrm{l} / \mathrm{min}$ for induction, $1-2 \%$ at $0.4 \mathrm{l} / \mathrm{min}$ for maintenance) and appropriate analgesia was achieved by subcutaneous injection of Buprenorphine $(0.1 \mathrm{mg} / \mathrm{kg} \mathrm{BW}) 30$ minutes prior to surgery. Depth of anesthesia was monitored regularly by the absence of reflexes (hind limb withdrawal) and adjusted accordingly. During all experiments, animals were placed on a heating pad and body temperature was maintained at $37^{\circ} \mathrm{C}$. All experimental procedures were done in compliance with the German national animal care guidelines and approved by the local animal welfare committee of the University Medical Center Göttingen as well as the animal welfare office of the state of Lower Saxony, Germany (LAVES).

Virus purification: Adeno-associated viruses (AAVs) were generated in HEK-293T cells (ATCC) using polyethylenimine transfection ( $25.000 \mathrm{MW}$, Polysciences, USA) ${ }^{35,36}$. In brief, triple transfection of HEK-293T cells was performed using pHelper plasmid (TaKaRa/Clontech), trans-plasmid providing viral capsid PHP.B (generous gift from Ben Deverman and Viviana Gradinaru, Caltech, USA). The cell line was regularly tested for mycoplasma. We harvested viral particles $72 \mathrm{~h}$ after transfection from the medium and $120 \mathrm{~h}$ after transfection from cells and the medium. Viral particles from the medium were precipitated with $40 \%$ polyethylene glycol 8000 (Acros Organics, Germany) in $500 \mathrm{mM} \mathrm{NaCl}$ for $2 \mathrm{~h}$ at $4^{\circ} \mathrm{C}$ and then after centrifugation at 4,000 g for 30 min combined with cell pellets for processing. The cell pellets were suspended in $500 \mathrm{mM} \mathrm{NaCl}, 40 \mathrm{mM}$ Tris, $2.5 \mathrm{mM}$ $\mathrm{MgCl} 2, \mathrm{pH} 8$, and $100 \mathrm{U} \mathrm{mL}^{-1}$ of salt-activated nuclease (Arcticzymes, USA) at $37^{\circ} \mathrm{C}$ for $30 \mathrm{~min}$. Afterwards, the cell lysates were clarified by centrifugation at 2,000 $\mathrm{g}$ for $10 \mathrm{~min}$ and then purified over iodixanol (Optiprep, Axis Shield, Norway) step gradients (15\%, $25 \%, 40 \%$ and $60 \%)^{37,38}$ at $350,000 \mathrm{~g}$ for $2.25 \mathrm{~h}$. Viruses were concentrated using Amicon filters (EMD, UFC910024) and formulated in sterile phosphate-buffered saline (PBS) supplemented with $0.001 \%$ Pluronic F-68 (Gibco, Germany). Virus titers were measured using AAV titration kit (TaKaRa/Clontech) according to manufacturer's instructions by determining the number of DNase I resistant vg using qPCR (StepOne, Applied Biosystems). Purity of produced viruses was routinely checked by silver staining (Pierce, Germany) after gel electrophoresis (Novex ${ }^{\mathrm{TM}} 4-12 \%$ Tris-Glycine, Thermo Fisher Scientific) according to manufacturer's instruction. The presence of viral capsid proteins was positively confirmed in all virus preparations. Viral stocks were kept at $-80^{\circ} \mathrm{C}$ until injection. 
Virus injections: Viral vectors used in this study were either AAV-2/6 or the recently engineered AAV-PHP.B ${ }^{36}$. Vectors carried plasmids that code for the Channelrhodopsin-2-variant CatCh linked to the reporter-protein eYFP under control of the human synapsin promotor (titer: AAV2/6: $3.2 \times 10^{12}-2.7 \times 10^{13} \mathrm{GC} / \mathrm{ml} ;$ PHP.B: $4.57 \times 10^{12} \mathrm{GC} / \mathrm{ml}$ ). Injections were performed using micropipettes (20 $\mu \mathrm{m}$ tip diameter) pulled from quartz capillaries on a P-2000 laser puller (Sutter Instruments) connected to a pressure microinjector (100 - 125 PSI, PLI-100 pico-injector, Harvard Apparatus). 2-3 $\mu \mathrm{l}$ of virus suspension were injected directly into the left spiral ganglion of adult gerbils under general anesthesia using a recently developed intramodiolar approach ${ }^{20,39}$. After making an incision behind the ear, muscles and connective tissue covering the bulla tympanica were displaced and a bullostomy was performed in order to access the cochlea. Using a KFlex dental file, a small hole was then drilled into the basal part of the modiolus via the dorsal part of the round window niche to directly access the spiral ganglion. After injection, muscles and connective tissue were repositioned and the skin was sutured. Animals were allowed to recover for at least 4 weeks after surgery before continuing experiments. Positively transfected animals showed no significant differences in both thresholds (AAV2/6: $3.49 \pm 3.03 \mathrm{~mW}$, PHP.B: $1.70 \pm 0.99$ $\mathrm{mW}$, mean/SD, $p=0.073$, two-sample t-test) and maximal strength of responses (AAV2/6: $4.27 \pm$ $0.80 \mathrm{~d}^{\prime}$ values, PHP.B: $4.55 \pm 1.11 \mathrm{~d}$ ' values, mean/SD, $p=0.57$, two-sample t-test) that could be evoked in the inferior colliculus dependent on the virus they were injected with (Supplementary Figure 13).

Stimulation: Stimuli were generated and presented via a custom-made system based on NI-DAQCards (NI PCI-6229; National Instruments; Austin, US) controlled with custom-written MATLAB scripts (The MathWorks, Inc.; Natick, US). Acoustic stimuli were presented near field via a loudspeaker (Scanspeak Ultrasound; Avisoft Bioacoustics, Glienicke, Germany) centered $30 \mathrm{~cm}$ in front of the animals' head. A 0.25-inch microphone and measurement amplifier (D4039; 2610; Brüel \& Kjaer $\mathrm{GmbH}$, Naerum, Denmark) were used to calibrate sound pressure levels. For optical stimulation, access to the cochlea was achieved using the surgical approach described for virus injections. An optical fiber (200 $\mu \mathrm{m}$ diameter, 0.39 NA; Thorlabs, Dachau, Germany) coupled to a blue laser (473 nm, 100 mW DPSS; Changchun New Industry Optoelectronics) was then inserted into the cochlea either via the round window (for basal stimulation) or via cochleostomies in the middle or apical cochlear turn, respectively (stimulation sites in which the cochleostomy resulted in bleeding have been excluded from analysis; 3/29 STCs). Radiant flux from the fiber aperture was calibrated with a power meter before each experiment (Solo-2; Gentec-EO; München, Germany). Biphasic pulses (100 $\mu$ s phase duration) of varying electric current were generated with 
a custom-made current-source stimulus isolator and delivered via 4-channel (600 $\mu \mathrm{m}$ electrode spacing) rodent $\mathrm{eCl}$, provided by Roland Hessler, MED-EL Innsbruck (for details see ${ }^{40}$ ). The implant was inserted into the scala tympani via the round window, such that the most apical electrode was approximately 5-6 $\mathrm{mm}$ within the cochlea. Implant positioning was confirmed by our physiological results: Considering $11 \mathrm{~mm}$ length of the gerbil basilar membrane and a hearing range of $\sim 0.2-50 \mathrm{kHz}$, the $\mathrm{eCl}$ would cover approx. 5.5/11 mm (=50\%) of the cochlear length, which corresponds - according to the Greenwood function - to a CF at the cochlear location at the tip of the eCl of approximately $\left(f=0.39\left(10^{2.1 x}-0.5\right)=4.18 \mathrm{kHz}{ }^{28,41}\right.$. The mean CF recorded in response to electrical stimulation at electrode 1 (which is located at the very tip of the implant) was $3.72 \mathrm{kHz}$ in response to monopolar and $4.5 \mathrm{kHz}$ in response to bipolar stimulation (Supplementary Figure 7). The return electrode for monopolar electrical stimulation was placed between connective tissue and the bone outside of the bulla tympanica. The return electrode for bipolar stimulation was chosen as the neighboring electrode to the stimulation electrode in basal direction.

Recording of Multi-Unit Activity (MUA): To access the inferior colliculus (IC), an incision was made in the animal's scalp along the midline of the skull. After cleaning the bone, a thin layer of selfetching UV glue (Orbi-Bond; Orbis Dental, Münster, Germany) was applied and a head post was mounted rostrally to bregma using dental cement (Paladur; Kulzer, Hanau, Germany). The animal's head was fixed and bregma and lambda were aligned stereotactically. A low impedance $(<1 \Omega)$ metal wire was implanted between the skull and the cortical surface on the left hemisphere to serve as a reference electrode. Using a dental drill, a craniotomy ( $1 \mathrm{~mm}$ diameter; centered $2 \mathrm{~mm}$ lateral and $0.5 \mathrm{~mm}$ caudal to lambda) was performed on the right hemisphere of the animal's skull in order to access the IC contralateral to the injected ear. After removing the dura over primary visual cortex (which partly covers the gerbil's IC ${ }^{42}$ ) with a sharp needle, a linear 32-electrode silicon probe (177 $\mu^{2}$ electrode surface, $50 \mu \mathrm{m}$ electrode spacing, 1-3 $\mathrm{M} \Omega$ impedance measured at $1 \mathrm{kHz}$; Neuronexus, Ann Arbor, US) was positioned above the brain 2 $\mathrm{mm}$ lateral to lambda and as close as possible to the transverse sinus (which also covers the IC in this species) as possible. Initially, the probe was slowly inserted $\sim 3.3 \mathrm{~mm}$ into the brain (measured from the surface of visual cortex) using a LN Junior 4RE micromanipulator (Luigs \& Neumann; Ratingen, Germany). After waiting for one hour in order to obtain a stable preparation, a first mapping of recording sites was done using acoustic stimuli at $60-80 \mathrm{~dB}$ ranging from $0.5-32 \mathrm{kHz}$. Based on the measured neuronal activity the probe was then further advanced (or retracted) in order to optimally access the tonotopic axis of the central nucleus of the IC (ICC; ${ }^{24,43}$ ) and to be 
able to compare neuronal responses to optogenetic/electrical stimulation across animals later on. Once the silicon probe was positioned, activity of multi-neuronal clusters was amplified, filtered $(0.1-9,000 \mathrm{~Hz})$ and recorded at a sampling rate of $32 \mathrm{kHz}$ using the Digital Lynx $4 \mathrm{~S}$ recording system (Neuralynx; Dublin, Ireland). Data was stored on a hard drive and analyzed off-line.

Event extraction: All data was analyzed using custom-written MATLAB scripts. To obtain time stamps of neuronal events, thresholds were set manually on $0.6-6 \mathrm{kHz}$ bandpass-filtered $\left(4^{\text {th }}\right.$ order Butterworth-filter) data traces, usually at the level of three times the median absolute deviation of the whole data trace. Each crossing of this threshold was considered a time stamp of a neuronal event and a refractory time of 1 ms was implemented after each time stamp. For electrical stimulation, a linear interpolation was performed from the sampling point just before trigger onset to the sampling point $3 \mathrm{~ms}$ after trigger onset before thresholding in order to remove the electrical artefact from the data trace (Supplementary Figure $14 \mathrm{~A}-\mathrm{C}$ ). The artefact removal has subsequently been verified with data recorded from dead animals where no neuronal component could be observed in addition to the artefact (Supplementary Figure 14D-H). Frequency tuning: Frequency response areas were constructed in response to pure tones $(100 \mathrm{~ms}$ duration, $5 \mathrm{~ms}$ sine squared ramps for stimulus on- and offset, $150 \mathrm{~ms}$ inter-stimulus interval) ranging from $0.5-32 \mathrm{kHz}$ in quarter octave steps at sound pressure levels ranging from 10-80 $\mathrm{dB}$ SPL in $10 \mathrm{~dB}$ steps. 20-30 repetitions of each frequency-intensity combination were presented in a pseudo-random order, where each stimulus was presented once before presenting the next iteration of trials. The characteristic frequency (CF) and its corresponding threshold at each recording site was determined as the frequency that elicited responses at the lowest sound pressure level during the period of stimulus presentation ${ }^{44,45}$.

Responses to artificial SGN stimulation: To determine a time window in which potential responses to optical or electrical stimulation can be evaluated, a peri-stimulus time histogram (PSTH; 0.05 ms bin size) was constructed from pooled multi-unit activity recorded at all recording sites in

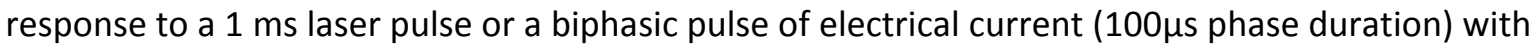
the maximal stimulus intensity presented $(\sim 35 \mathrm{~mW}, 500 \mu \mathrm{A})$. The response was defined as MUA exceeding the mean spike rate plus 3 standard deviations $20 \mathrm{~ms}$ before stimulus onset and was observed 2.25-24.5 ms after stimulus onset for optical stimulation and 2.45-13.25 ms after stimulus onset for electrical stimulation (Supplementary Figure 2). Based on these results, the response windows were set to $0-25 \mathrm{~ms}$ after stimulus onset for optical and to 0-14 ms after stimulus onset for electrical stimulation. 
Spatial spread of excitation: To quantify response thresholds and the spread of excitation, spatial tuning curves (STC) based on the cumulative discrimination index (d-prime; $d^{\prime}$ ) of spike rates were constructed $^{7,21,22}$ : For each electrode, the distribution of spikes during 20-30 trials in response to one stimulus was compared against the distribution of spikes during each trial in response to the subsequent (higher intensity) stimulus, where stimuli are sorted according to their intensity, starting with an intensity of zero (no stimulus condition). A receiver operating characteristic (ROC) curve was constructed from these distributions. The area under the ROC curve, which depicts the Z-score (measured in standard deviations), was then multiplied by $\sqrt{2}$ in order to obtain the $d^{\prime}$ value. $D^{\prime}$ values of successively increasing stimulus intensities were finally summed up in order to calculate the cumulative discrimination index. In the next step, a matrix was constructed by sorting cumulative $d^{\prime}$ values according to the electrode position they were obtained from in one dimension and the stimulus intensity they were evoked by in the other dimension. Iso- $d$ '-contourlines were then interpolated by using MATLABs built-in contour function and thresholds of neuronal activation were determined as the stimulus intensities that correspond to isolines at the cumulative $d^{\prime}$ level of 1 . The best electrode (BE) was defined as the electrode which showed the lowest threshold $\left(d^{\prime}=1\right)$. The spread of excitation was accessed as the distance between the most dorsal and the most ventral electrode with a $d^{\prime}>=1$ at the stimulus intensity that elicited a $d^{\prime}$ value of $1.5,2,2.5$ or 3 at the best electrode.

Monte Carlo ray tracing: Monte Carlo ray tracing simulation was performed using TracePro ${ }^{\circledR}$ Standard 7.8.1 (Lambda Research Corporation) to validate the experimentally used fiber stimulation. Briefly, different cochlear compartments were reconstructed from x-ray tomography and embedded in a solid cube to simulate bone enclosure. Each of the structures was assigned with mean optical properties from cerebrospinal fluid, brain tissue and bone, respectively ${ }^{46}$. All the light sources were modelled as the optical fiber used in the experiments (Thorlabs FT200UMT, $0.39 \mathrm{NA}$ ) and defined in TracePro as grid sources with the following parameters: circular pattern of 3003001 rays (1001 rings, $\lambda=473 \mathrm{~nm}$, uniform total intensity of $10 \mathrm{~mW}$ ); grid boundary radius: $100 \mu \mathrm{m}$; symmetric Gaussian spatial and angular beam distribution (waist radius of Gaussian beam profile: $100 \mu \mathrm{m}$; half angle of angular profile of the beam: $\left.16.79^{\circ}\right)$. Emitter surfaces were calculated for every position: First, the tonotopic axis was defined along the center line of Rosenthal's canal, where 300 query points were extrapolated and the corresponding frequency positions were calculated by the Greenwood function for a hearing range of $50-0.25 \mathrm{kHz}$ ( $f=0.39\left(10^{2.1 x}-0.5\right)$, where $\mathrm{x}$ is the cochlear length normalized from 0 to 1 in baso-apical 
direction). Second, coordinates for the tips of optical fibers were placed in anatomically meaningful positions corresponding to fiber placement in our in vivo experiments. These coordinates were then translated following a straight line to Rosenthal's canal to a given distance from the query point $(400,700$ and $900 \mu \mathrm{m}$ for apical, mid-cochlear and basal stimulation, respectively). The newly calculated coordinate was defined as the origin of the light source and the straight line as its normal vector. To account for variability in fiber placement during our experiments, two models were calculated. In the rotation model, the normal vector was rotated \pm 15 degrees in two perpendicular. In the translational model, the normal vector was coaxially translated \pm 100 and $200 \mu \mathrm{m}$. The origin and the normal vector of all five sources (initial position plus 4 rotations or four translations) at the three positions were imported to TracePro. Radiant flux was read from the 300 query points (included as solid spheres with a $5 \mu \mathrm{m}$ radius, with assigned optical properties of brain tissue). Irradiance was calculated as radiant flux $/ 4 * \mathrm{pi}^{*}$ radius $^{2}$. Irradiance values were linearly scaled to $2.67 \mathrm{~mW}$, which was the mean threshold for optogenetic stimulation observed in our experiments (Supplementary Figure 5). The mean irradiance profile was calculated for every position using the irradiance profile of all five sources, and the tonotopic location the fiber was facing was then calculated as the peak of the mean irradiance profile.

IC histology: After each experiment the silicon probe was retracted with the micromanipulator and a tungsten electrode covered with Dil (DilC18(3); Thermo Fisher Scientific, Darmstadt, Germany) was inserted into the IC at the same site in order to stain the electrodes' position. Afterwards brains were explanted and fixed in 4\% PFA in phosphate buffered saline (PBS) for several days before they were moved to $30 \%$ sucrose in PBS for cryoprotection. Coronal slices of $50 \mu \mathrm{m}$ thickness were obtained using a Leica CM 3050 Cryostate $\left(-25^{\circ} \mathrm{C}\right.$ object temperature). Slices were mounted on microscope slides using Fluoroshield mounting medium (Sigma Aldrich, Darmstadt, Germany), which contains DAPI in order to stain the cell nuclei.

Data Availability: Data generated and analyzed during the current study is available from the corresponding author upon reasonable request. Numerical source data underlying figures 1e, 2a$k, 4 b-j$, as well as supplementary figures $1,2,5,7,9,11,12,13$ are provided as a source data file. Code Availability: Analysis code - written in MATLAB 2016a - is available from the corresponding author upon reasonable request. 


\section{References}

1. Zeng, F.-G., Rebscher, S., Harrison, W. V., Sun, X. \& Feng, H. Cochlear Implants:System Design, Integration and Evaluation. IEEE Rev. Biomed. Eng. 1, 115-142 (2008).

2. Lenarz, T. Cochlear implant - state of the art. GMS Curr. Top. Otorhinolaryngol. Head Neck Surg. 16, Doc04 (2018).

3. Middlebrooks, J. C., Bierer, J. A. \& Snyder, R. L. Cochlear implants: the view from the brain. Curr. Opin. Neurobiol. 15, 488-493 (2005).

4. Kral, A., Hartmann, R., Mortazavi, D. \& Klinke, R. Spatial resolution of cochlear implants: the electrical field and excitation of auditory afferents. Hear. Res. 121, 11-28 (1998).

5. Friesen, L. M., Shannon, R. V., Baskent, D. \& Wang, X. Speech recognition in noise as a function of the number of spectral channels: Comparison of acoustic hearing and cochlear implants. J. Acoust. Soc. Am. 110, 1150 (2001).

6. Zeng, F.-G. \& Galvin, J. J. I. Amplitude Mapping and Phoneme Recognition in Cochlear Implant Listeners. Ear Hear. 20, (1999).

7. Richter, C.-P. et al. Spread of cochlear excitation during stimulation with pulsed infrared radiation: inferior colliculus measurements. J. Neural Eng. 8, 056006 (2011).

8. Hernandez, V. H. et al. Optogenetic stimulation of the auditory pathway. J. Clin. Invest. 124, 1114-1129 (2014).

9. Moser, T. Optogenetic stimulation of the auditory pathway for research and future prosthetics. Curr. Opin. Neurobiol. 34, 29-36 (2015).

10. Jeschke, M. \& Moser, T. Considering optogenetic stimulation for cochlear implants. Hear. Res. 322, 224-234 (2015).

11. Izzo, A. D. et al. Selectivity of neural stimulation in the auditory system: a comparison of optic and electric stimuli. J. Biomed. Opt. 12, 021008 (2007).

12. Izzo, A. D. et al. Laser stimulation of auditory neurons: effect of shorter pulse duration and penetration depth. Biophys. J. 94, 3159-3166 (2008).

13. Young, H. K., Tan, X., Xia, N. \& Richter, C.-P. Target structures for cochlear infrared neural stimulation. Neurophotonics 2, 025002 (2015).

14. Kallweit, N. et al. Optoacoustic effect is responsible for laser-induced cochlear responses. Sci. Rep. 6, (2016).

15. Schultz, M. et al. Nanosecond laser pulse stimulation of the inner ear-a wavelength study. Biomed. Opt. Express 3, 3332-3345 (2012). 
16. Nagel, G. et al. Channelrhodopsin-2, a directly light-gated cation-selective membrane channel. Proc. Natl. Acad. Sci. 100, 13940-13945 (2003).

17. Boyden, E. S., Zhang, F., Bamberg, E., Nagel, G. \& Deisseroth, K. Millisecond-timescale, genetically targeted optical control of neural activity. Nat Neurosci 8, 1263-1268 (2005).

18. Keppeler, D., Merino, C. \& Lopez, D. Ultrafast optogenetic stimulation of the auditory pathway by targeting-optimized Chronos. EMBO J. (2018).

19. Mager, T. et al. High frequency neural spiking and auditory signaling by ultrafast redshifted optogenetics. Nat. Commun. 9, 1750 (2018).

20. Wrobel, C. et al. Optogenetic stimulation of cochlear neurons activates the auditory pathway and restores auditory-driven behavior in deaf adult gerbils. Sci. Transl. Med. 10, eaao0540 (2018).

21. Middlebrooks, J. C. \& Snyder, R. L. Auditory prosthesis with a penetrating nerve array. J. Assoc. Res. Otolaryngol. JARO 8, 258-279 (2007).

22. Snyder, R. L., Bierer, J. A. \& Middlebrooks, J. C. Topographic spread of inferior colliculus activation in response to acoustic and intracochlear electric stimulation. J. Assoc. Res. Otolaryngol. JARO 5, 305-322 (2004).

23. Zhu, Z., Tang, Q., Zeng, F.-G., Guan, T. \& Ye, D. Cochlear-implant spatial selectivity with monopolar, bipolar and tripolar stimulation. Hear. Res. 283, 45-58 (2012).

24. Schnupp, J. W. H., Garcia-Lazaro, J. A. \& Lesica, N. A. Periodotopy in the gerbil inferior colliculus: local clustering rather than a gradient map. Front. Neural Circuits 9, 37 (2015).

25. Harris, D. M., Shannon, R. V., Snyder, R. \& Carney, E. Multi-unit mapping of acoustic stimuli in gerbil inferior colliculus. Hear. Res. 108, 145-156 (1997).

26. Cant, N. B. \& Benson, C. G. Organization of the inferior colliculus of the gerbil (Meriones unguiculatus): Differences in distribution of projections from the cochlear nuclei and the superior olivary complex. J. Comp. Neurol. 495, 511-528 (2006).

27. Graña, G. D. et al. The organization of frequency and binaural cues in the gerbil inferior colliculus: GRAÑA et al. J. Comp. Neurol. 525, 2050-2074 (2017).

28. Dong, W. \& Olson, E. S. In vivo impedance of the gerbil cochlear partition at auditory frequencies. Biophys. J. 97, 1233-1243 (2009).

29. Nadol, J. B. Comparative anatomy of the cochlea and auditory nerve in mammals. Hear. Res. 34, 253-266 (1988).

30. Hatsushika, S., Shepherd, R. K., Tong, Y. C., Clark, G. M. \& Funasaka, S. Dimensions of the scala tympani in the human and cat with reference to cochlear implants. Ann. Otol. Rhinol. Laryngol. 99, 871-876 (1990). 
31. Semple, M. N. \& Kitzes, L. M. Single-unit responses in the inferior colliculus: different consequences of contralateral and ipsilateral auditory stimulation. J. Neurophysiol. 53, 1467-1482 (1985).

32. Allitt, B. J. et al. Midbrain responses to micro-stimulation of the cochlea using high density thin-film arrays. Hear. Res. 287, 30-42 (2012).

33. Allitt, B. J., Harris, A. R., Morgan, S. J., Clark, G. M. \& Paolini, A. G. Thin-film microelectrode stimulation of the cochlea in rats exposed to aminoglycoside induced hearing loss. Hear. Res. 331, 13-26 (2016).

34. Goßler, C. et al. GaN-based micro-LED arrays on flexible substrates for optical cochlear implants. J. Phys. Appl. Phys. 47, 205401 (2014).

35. Gray, S. J. et al. Production of Recombinant Adeno-Associated Viral Vectors and Use in In Vitro and In Vivo Administration. in Current Protocols in Neuroscience (eds. Crawley, J. N. et al.) (John Wiley \& Sons, Inc., 2011). doi:10.1002/0471142301.ns0417s57

36. Deverman, B. E. et al. Cre-dependent selection yields AAV variants for widespread gene transfer to the adult brain. Nat. Biotechnol. 34, 204-209 (2016).

37. Zolotukhin, S. et al. Recombinant adeno-associated virus purification using novel methods improves infectious titer and yield. Gene Ther. 6, 973-985 (1999).

38. Grieger, J. C., Choi, V. W. \& Samulski, R. J. Production and characterization of adenoassociated viral vectors. Nat. Protoc. 1, 1412-1428 (2006).

39. Chen, W. et al. Restoration of auditory evoked responses by human ES-cell-derived otic progenitors. Nature 490, 278-282 (2012).

40. Wiegner, A., Wright, C. G. \& Vollmer, M. Multichannel cochlear implant for selective neuronal activation and chronic use in the free-moving Mongolian gerbil. J. Neurosci. Methods 273, 40-54 (2016).

41. Huet, A. et al. Sound coding in the auditory nerve of gerbils. Hear. Res. 338, 32-39 (2016).

42. Cant, N. B. \& Benson, C. G. An atlas of the inferior colliculus of the gerbil in three dimensions. Hear. Res. 206, 12-27 (2005).

43. Ryan, A. F., Woolf, N. K. \& Sharp, F. R. Tonotopic organization in the central auditory pathway of the Mongolian gerbil: a 2-deoxyglucose study. J. Comp. Neurol. 207, 369-380 (1982).

44. Kiang, N. Y. S., Liberman, M. C. \& Baer, T. Tuning curves of auditory-nerve fibers. J. Acoust. Soc. Am. 61, S27-S27 (1977).

45. Egorova, M., Ehret, G., Vartanian, I. \& Esser, K. H. Frequency response areas of neurons in the mouse inferior colliculus. I. Threshold and tuning characteristics. Exp. Brain Res. 140, 145-161 (2001). 
46. Wrobel, C. et al. Optogenetic stimulation of cochlear neurons activates the auditory pathway and restores auditory-driven behavior in deaf adult gerbils. Sci. Transl. Med. 10, eaao0540 (2018). 
Acknowledgments: The authors are very grateful to Carolyn Garnham and Roland Hessler from MED-EL for providing cochlear implants for animal research. We thank Gerhard Hoch for hardand software development of multi-channel electrical stimulation and expert technical assistance. We thank Daniel Keppeler for providing the $\mathrm{x}$-ray images in supplementary figure S2 and providing an $\mathrm{x}$-ray tomography reconstructed gerbil cochlea for Monte-Carlo ray tracing. We thank Daniela Gerke for expert help with virus preparation. We thank Ulrich Schwarz for discussion regarding the dynamic range estimations. We thank Ben Deverman and Viviana Gradinaru for providing the PHP.B construct used in this study. The work was funded by the European Research Council (ERC) under the European Union's Horizon 2020 research and innovation program (grant agreement No 670759 - advanced grant "OptoHear") to TM. AD is a fellow of the German Academic Scholarship Foundation.

Author contributions: $A D, M J$ and TM designed the study. MJ designed hard- and software for multi-channel electrophysiological recordings. AD performed virus injections, IC recordings and IC histology. CDA performed Monte-Carlo ray trace modeling. VR produced the PHP.B-CatCh virus. $A D$ performed data analysis under the supervision of $M J$ and $T M . A D, M J$ and $T M$ prepared the manuscript.

Competing interests: The authors declare no competing interests. 


\section{Supplementary Information}

Supplementary Figures:

Supplementary Figure 1: Linear fit of tonotopic slopes

Supplementary Figure 2: Auditory thresholds of ICC multi-units

Supplementary Figure 3: Response window of artificial SGN stimulation

Supplementary Figure 4: Intracochlear optical stimulation of non-injected gerbils

Supplementary Figure 5: Thresholds for artificial SGN stimulation

Supplementary Figure 6: Multi-peak STCS

Supplementary Figure 7: Tonotopy of artificial SGN stimulation

Supplementary Figure 8: Verifying fiber positions by Monte-Carlo ray tracing

Supplementary Figure 9: Spatial spread of excitation

Supplementary Figure 10: Effect of fiber angle on spread of excitation

Supplementary Figure 11: Spectral spread $6 \mathrm{~dB}$ above threshold

Supplementary Figure 12: Saturation of stimulus-response functions

Supplementary Figure 13: Comparison of AAV2/6 and AAV-php.b injected animals

Supplementary Figure 14: Artefact removal of electrical stimulation 


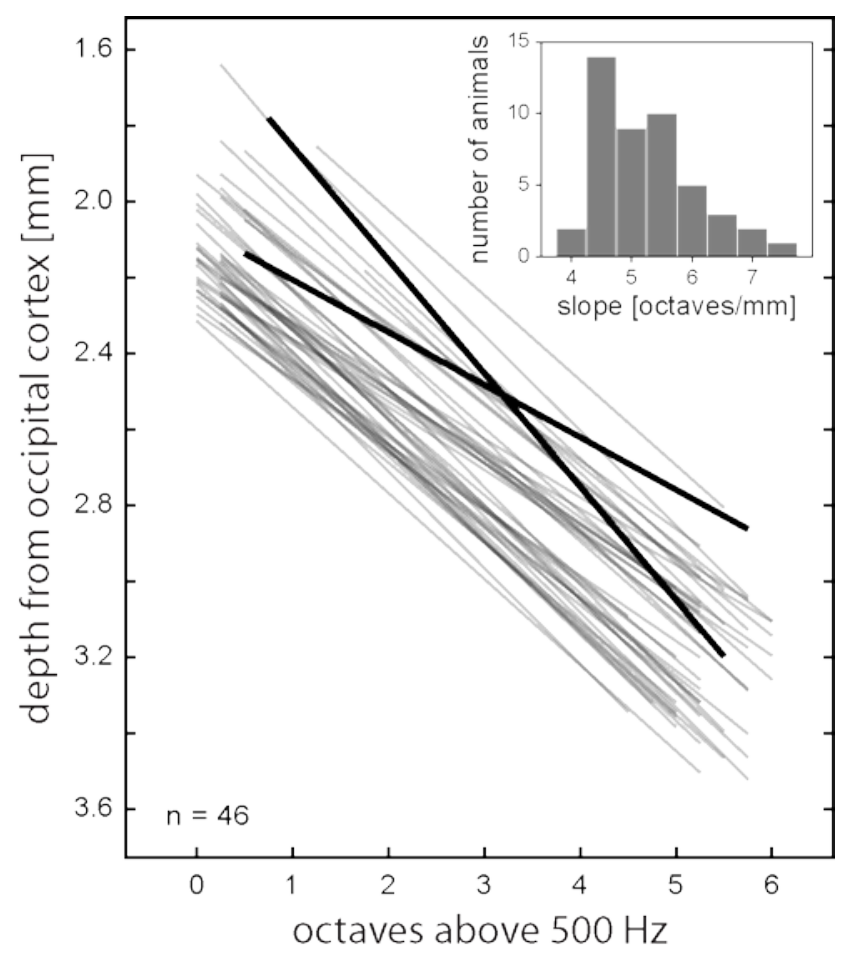

Supplementary Figure 1: Linear fit of tonotopic slopes. The tonotopic slope of each animal was calculated by a linear fit of the characteristic frequencies at given recording depths. The steepest and shallowest slopes are depicted in black lines. Inset: Distribution of tonotopic slopes in $\mathbf{4 6}$ gerbils contributing to this study. Source data is provided as a source data file. 


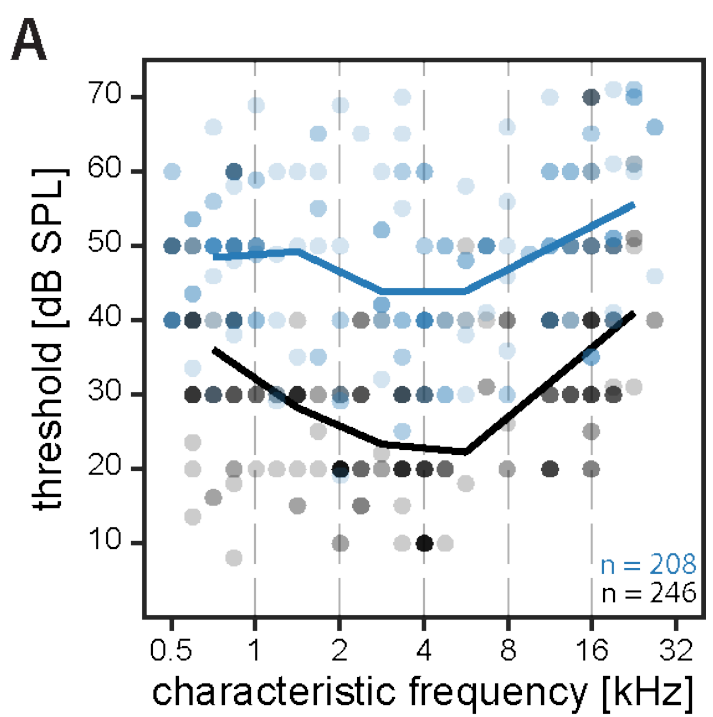

Supplementary Figure 2: Auditory thresholds of multi-units in the central nucleus of the inferior colliculus (ICC). (A) Thresholds of acoustically driven multi-units in non-injected animals without cochlear surgery (black) and in AAV-injected animals upon fiber insertion (blue). Solid lines show the mean threshold for each octave band. In animals that underwent cochlear surgery, thresholds of ICC multi-units were elevated by $\sim 20 \mathrm{~dB}$ SPL on average. For each octave band, $p$-values are below 0.001, at least (two-sample t-test). Source data is provided as a source data file. 


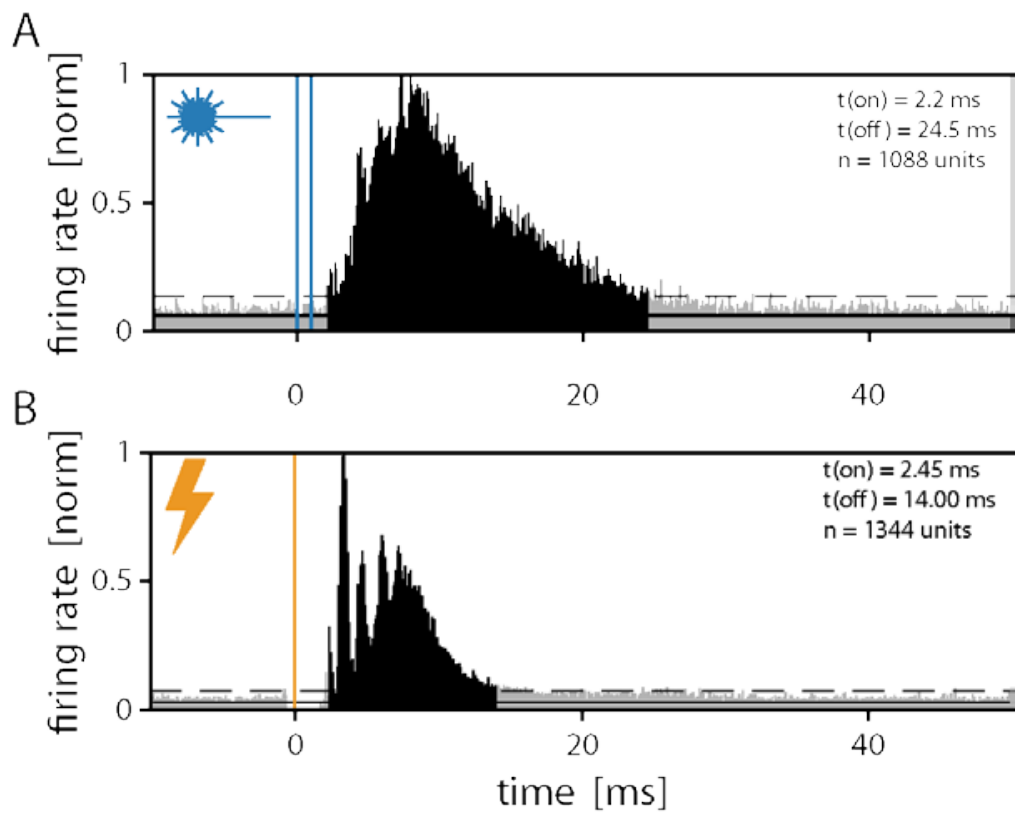

Supplementary Figure 3: Response window of artificial auditory nerve stimulation. (A) Peri-Stimulus-Time-Histogram (PSTH; normalized to 1) in response to optical stimulation via the round window. (B) PSTH (normalized to 1) in response to electrical stimulation via a cochlear implant inserted into the scala tympani via the round window. Both PSTHs are compiled across all multiunits that have been recorded upon optical or electrical stimulation. Blue and orange lines indicate stimulus on- and offset, respectively. Solid and dashed black lines indicate mean firing rate as well as the mean firing rate plus 3 standard deviations, respectively. The detected times of response on- and offsets ared epicted in the corresponding panel. 


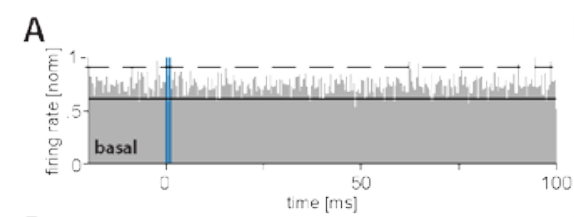

$\mathrm{D}$

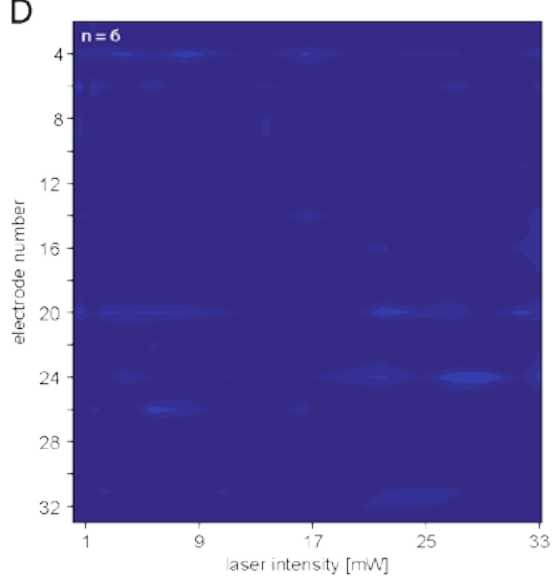

B

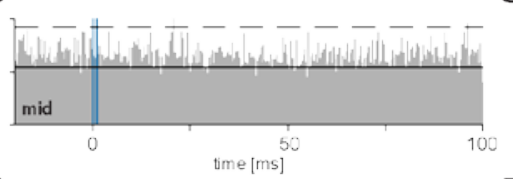

E

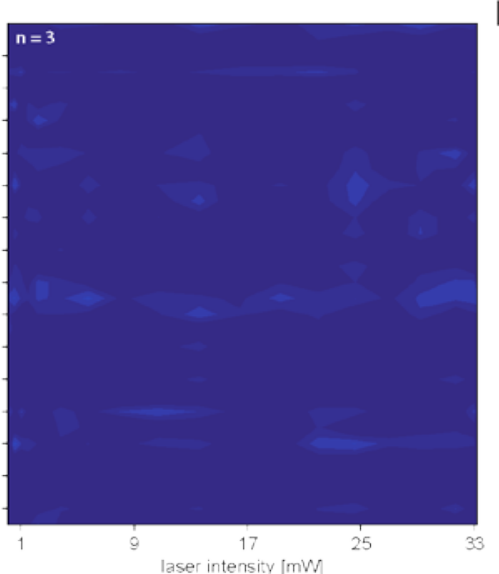

$C$
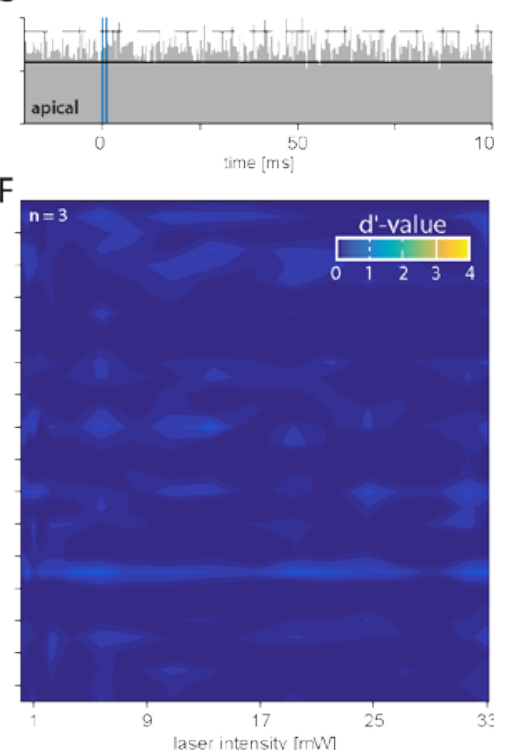

Supplementary Figure 4: Intracochlear optical stimulation of non-injected gerbils. (A-C) Peri-Stimulus-Time-Histogram (normalized) in response to optical stimulation via the round window ( $\mathrm{A} ; \mathrm{n}=6$ gerbils) and cochleostomies in the middle ( $B ; n=3$ gerbils) and apical ( $C ; n=3$ gerbils) cochlear turn. Blue lines indicate stimulus on- and offset, respectively. Solid and dashed black lines indicate the mean firing rate as well as the mean firing rate plus 3 standard deviations, respectively. (D-F) Averaged spatial tuning curves (STCS) in response to basal (D) mid-turn (E) and apical (F) cochlear stimulation in non-injected animals. No responses ( $d^{\prime}>=1$ ) were observed in any individual STC. 

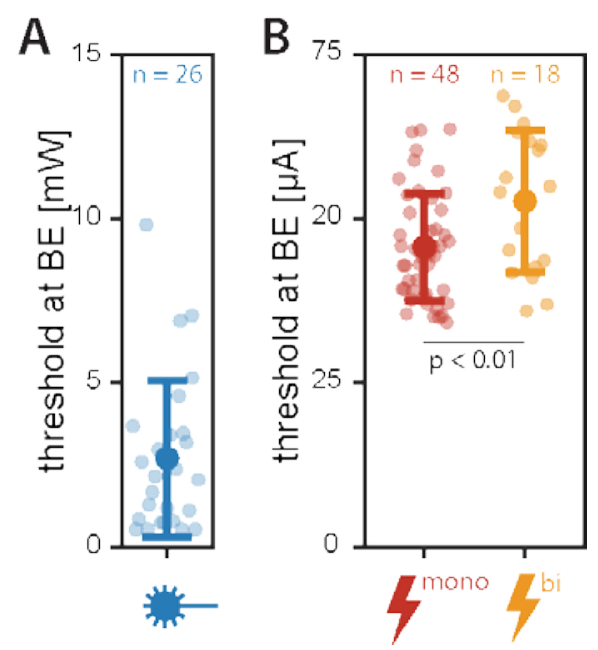

Supplementary Figure 5: Thresholds for artificial auditory nerve stimulation. (A) Thresholds of the best electrode (BE) for optically driven multi-units in the inferior colliculus (IC): Data is displayed as mean \pm s.d..

(B) Thresholds of the BE for electrically driven multi-units in the IC for monopolar (red) and bipolar (orange) stimulation ( $p<0.01$, twosample t-test). Data is displayed as mean \pm s.d.. Source data is provided as a source data file. 

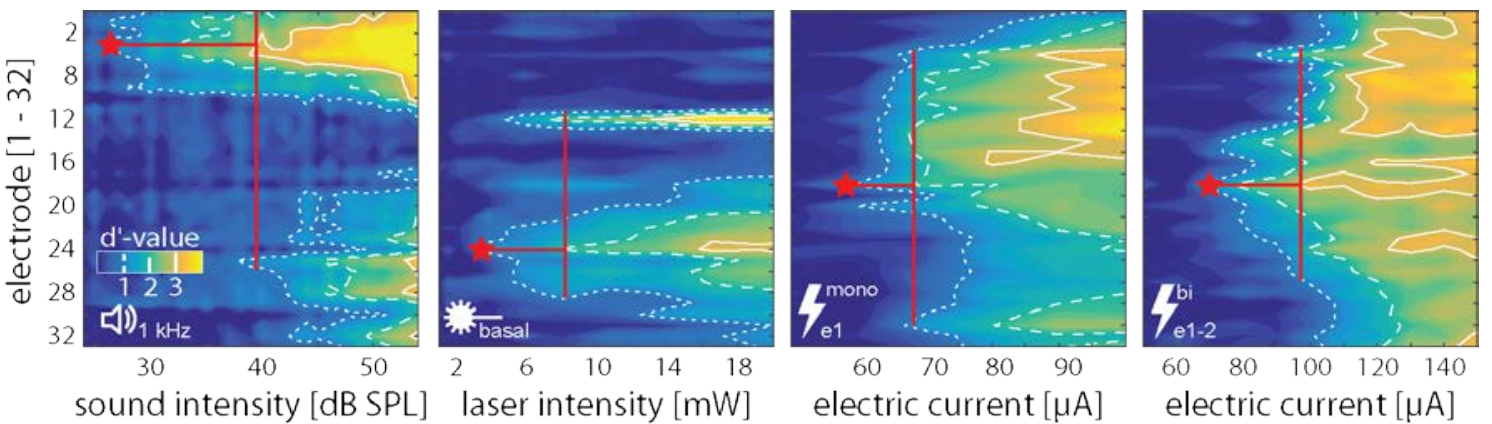

Supplementary Figure 6: Multi-peak spatial tuning curves (STCS). Few STCs with more than one peak have been observed for acoustic (left), optogenetic (center left), monopolar (center right) and bipolar (right) electrical stimulation. In these cases, the most dorsal and most ventral electrodes with significant responses have been considered as the boundaries of the STC in order not to underestimate the spread of excitation. 

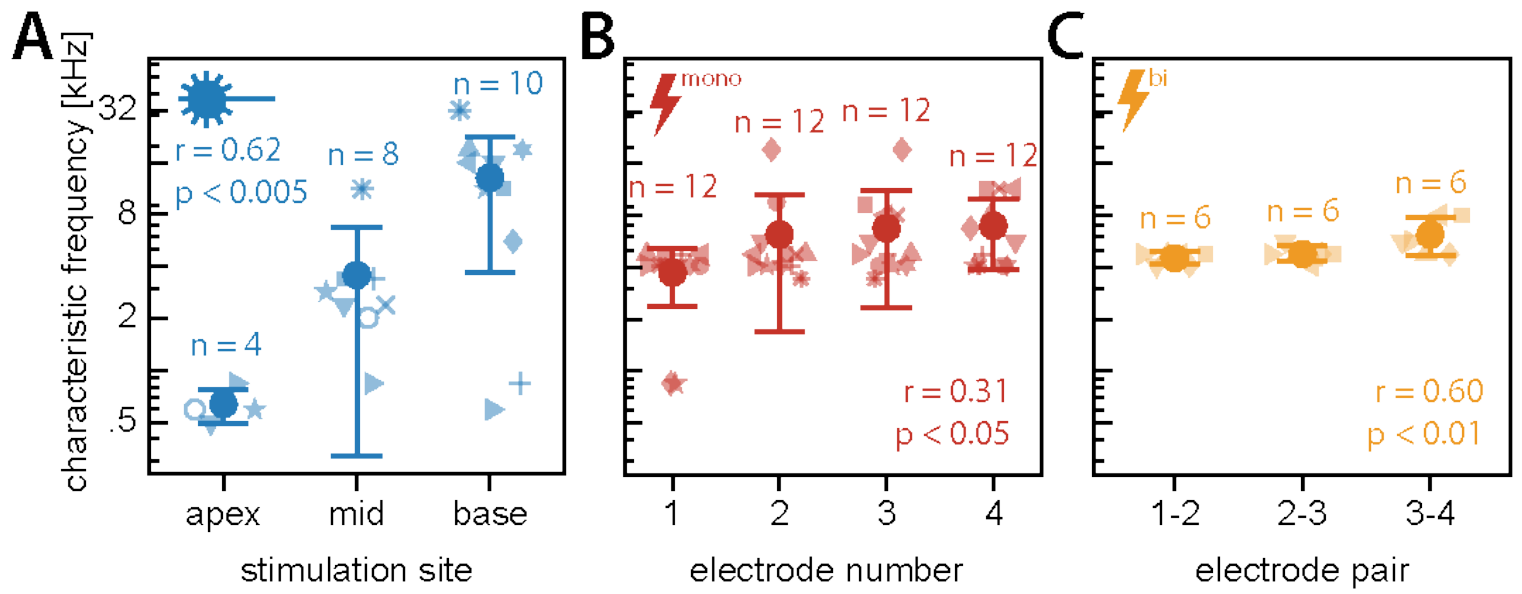

Supplementary Figure 7: Tonotopy of artificial auditory nerve stimulation. Characteristic frequencies (CFs) as a function of optical stimulation site in AAV-injected animals (A), stimulation electrode for monopolar electrical stimulation (B) and electrode pair for bipolar electrical stimulation (C) in non-injected animals. Data is displayed as mean and standard deviation for each stimulation site. Symbols mark different animals. Pearson's correlation coefficient $r$ and the corresponding $p$-values were calculated. The two low-frequency units in response to optical stimulation from the cochlear base most likely were positioned at the border - or even outside - of the central nucleus of the inferior colliculus (ICC), where low-frequency units typically are found. However, the neural excitation upon basal cochlear stimulation always had a focus in the ventral inferior colliculus (as shown when plotting best electrodes (BEs) in dependence of stimulation site; see Fig. 4C). The discrepancy in number of data points for apical and basal optical stimulation when compared to Fig. $4 \mathrm{C}$ is explained by an increase in auditory thresholds upon cochlear surgery (see fig. S2), so that acoustically driven activity could not be evoked at all BEs and thus the CFs could not be determined. This primarily affected multi-units coding for frequencies at the edge of the audiogram, which typically have higher thresholds than the ones in the mid-frequency range. Source data is provided as a source data file. 

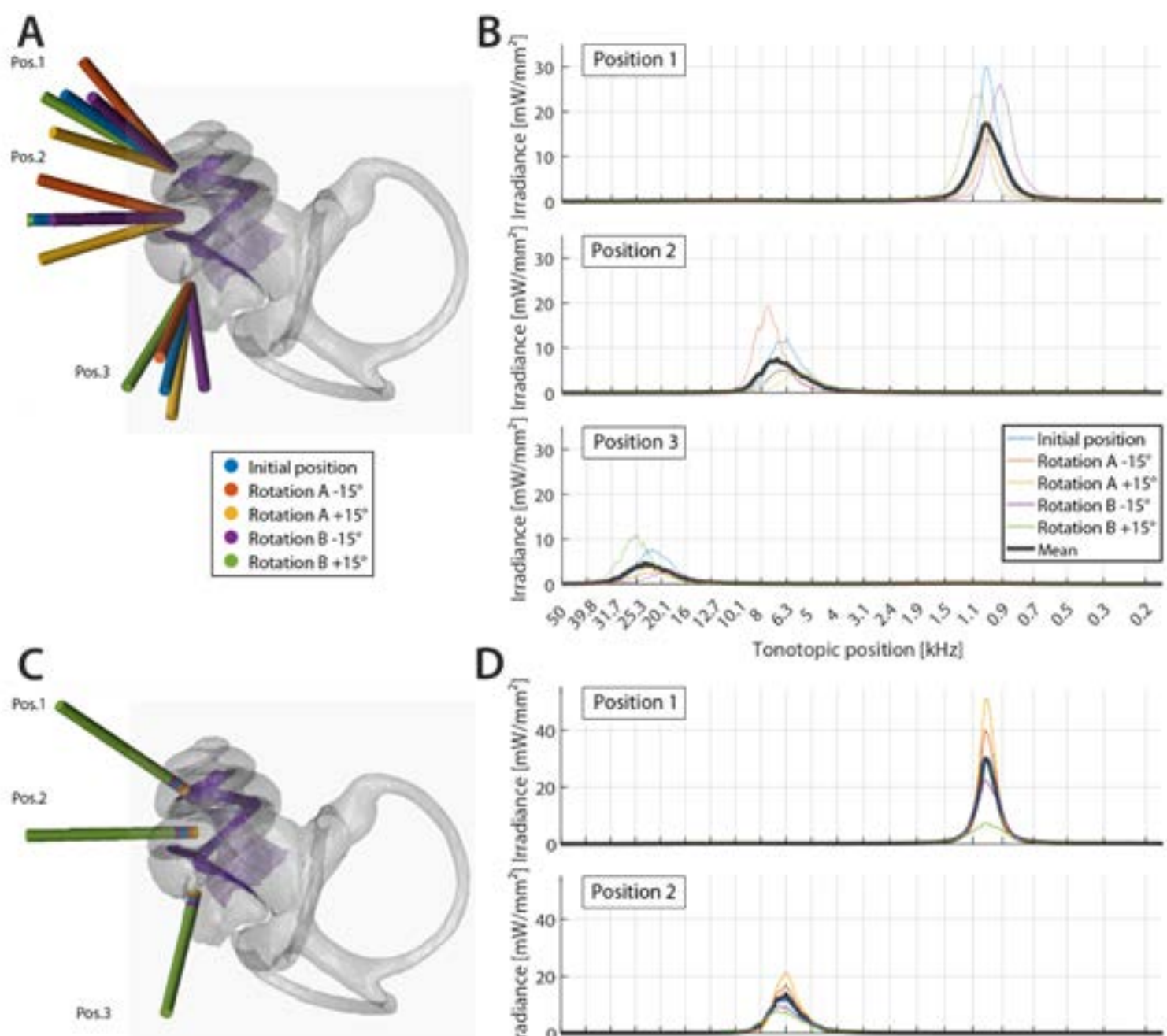

D

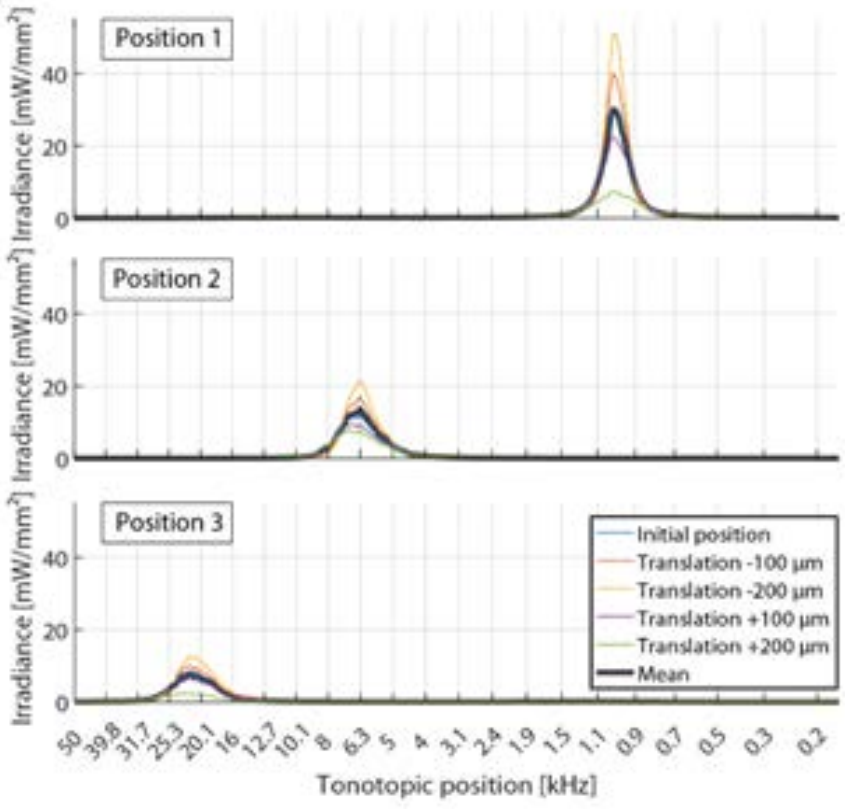

Supplementary Figure 8: Verifying fiber positions by Monte-Carlo ray tracing. 3D model reconstructed from x-ray tomography including fiber positions (Pos. 1-3), roughly corresponding to the ones used in physiological experiments. In the model, original positions as well as their respective rotation in two orthogonal planes ( $A$ and $B$, panel $A$ ) and four different coaxial translations (C) have been used to account for variability in fiber placement. Light grey: Bone; Grey: Scala media, vestibuli and tympani, as well as semicircular canals; Purple: Peripheral processes and Rosenthal's canal that houses the spiral ganglion neuron's somata; Fiber position and their corresponding rotations and translations, respectively, are indicated by different colors (see legend). (B/D) Irradiance profiles obtained from 300 query points located along the tonotopic axis, defined in the centerline of the Rosenthal's canal, upon Monte-Carlo ray tracing from the three different fiber positions using a source radiant flux of $2.67 \mathrm{~mW}$. Peak irradiances of the mean traces indicate that fibers from these different positions were stimulating areas around 1.01, 6.9 and $22.89 \mathrm{kHz}$, according to the rotation model in panel $\mathrm{B}$, and $1.02,6.34$ and 21.81 according to the translational model in panel D. Source data of panel $B$ and $D$ is provided as a source data file. 


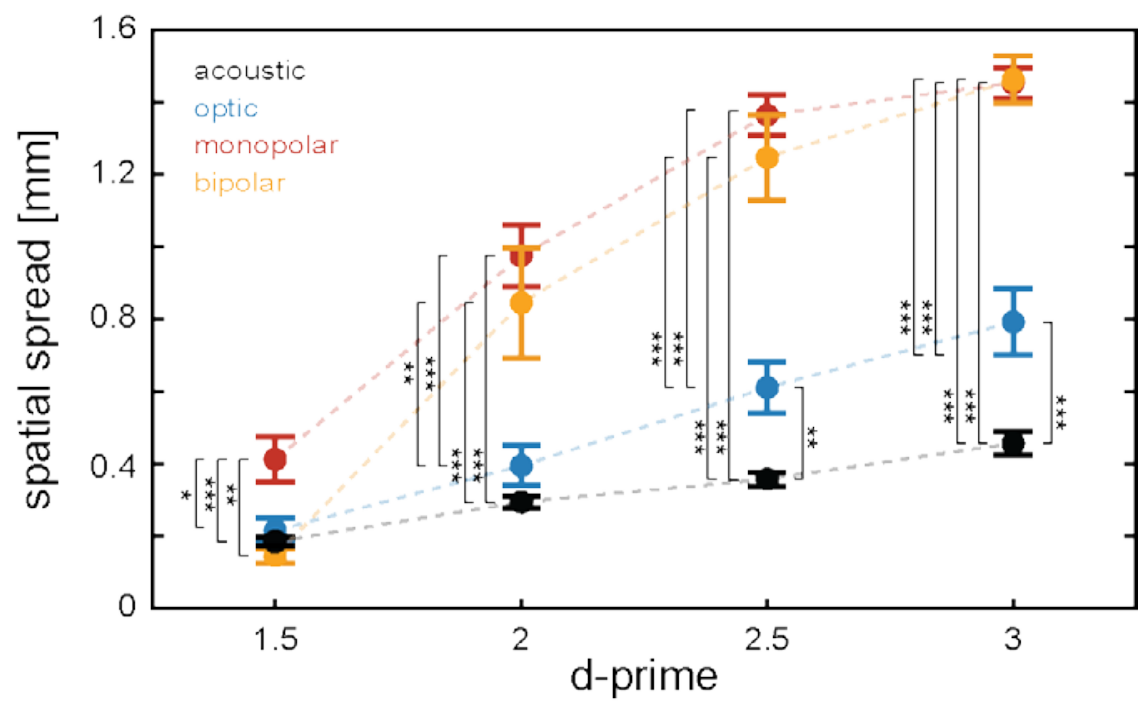

Supplementary Figure 9: Spatial spread of excitation. Mean and s.e.m. for the spatial spread of excitation upon acoustic, optogenetic, monopolar and bipolar electric stimulation. Stars indicate statistical significance (one star: $p<0.05$, two stars: $p<0.01$, three stars: $p<0.001$ ), according to a repeated-measures Anova and post-hoc pairwise comparison tests. Only significant differences have been indicated. Source data is provided as a source data file. 

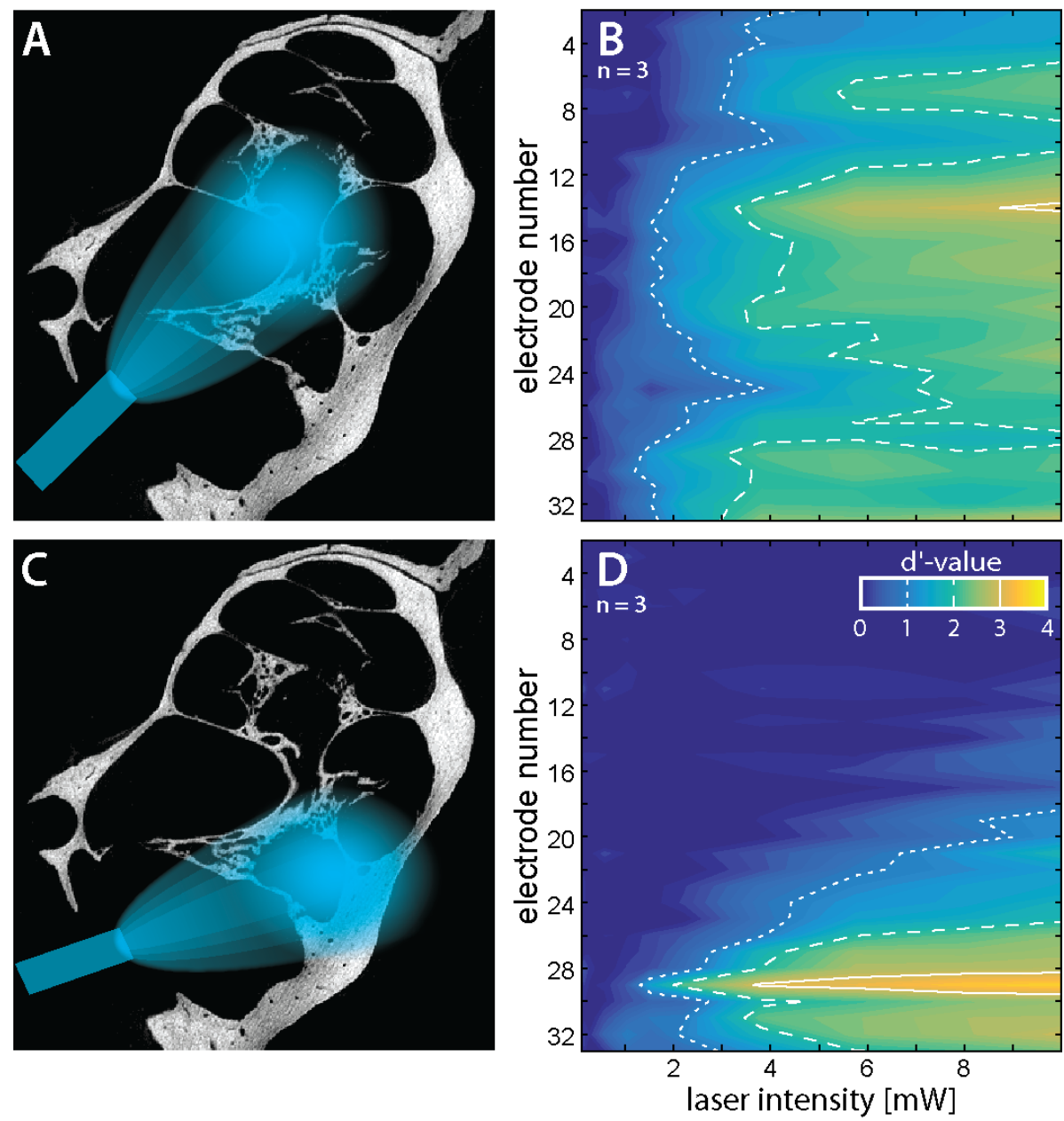

Supplementary Figure 10: Effect of fiber angle on spread of excitation. (A) Scheme of fiber placement and (B) resulting spread of excitation when the fiber aperture points towards the cochlear apex. (C) Positioning of the fiber aperture towards the cochlear base lead to more restricted spread of excitation (D). 


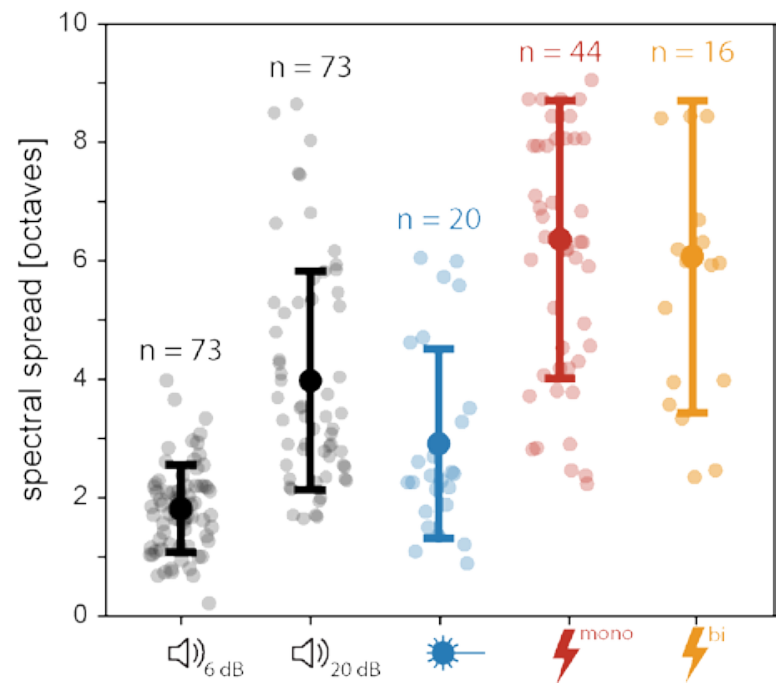

Supplementary Figure 11: Spectral spread $x$ dB above threshold. Spectral spread in octaves, measured $6 \mathrm{~dB}$ above threshold of the best electrode, as done in a different study by Middlebrooks and Snyder(Middlebrooks and Snyder, 2007) as well as $20 \mathrm{~dB}$ above threshold for acoustic stimulation, as done by Snyder, Bierer and Middlebrooks(Snyder et al., 2004). Data is depicted as mean \pm s.d.. Source data is provided as a source data file. 

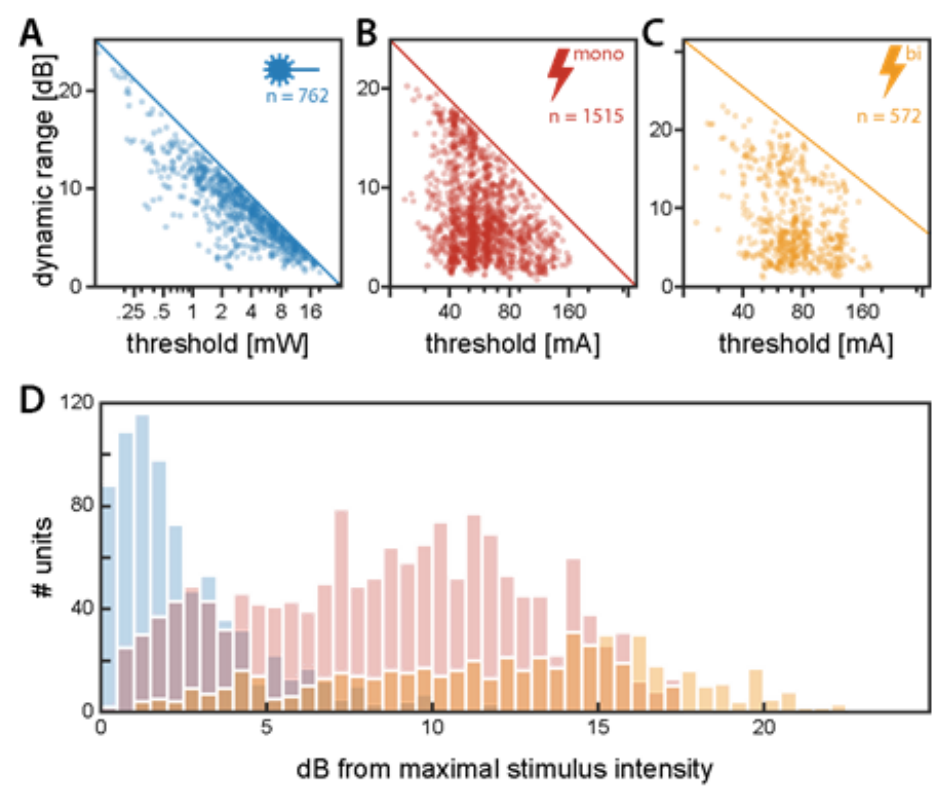

Supplementary Figure 12: Saturation of stimulus-response functions. Dynamic range (DR) of individual multi-units for optical (A), monopolar (B) and bipolar (C) electrical stimulation as a function of their respective thresholds (10\% intercept). Solid lines mark the largest DR possible at the respective threshold, limited by the setup's highest stimulus intensity. While optically driven units with higher thresholds (>2 $\mathrm{mW}$ ) seem to be mainly limited by hardware restrictions, this was not the case for electrically driven units. (D) Difference of saturated responses (90\% intercept) and the highest achievable DR (in dB). While optically driven multi-units tend to approach the highest achievable DR, electrically driven units typically were saturated at lower intensities. Source data is provided as a source data file. 

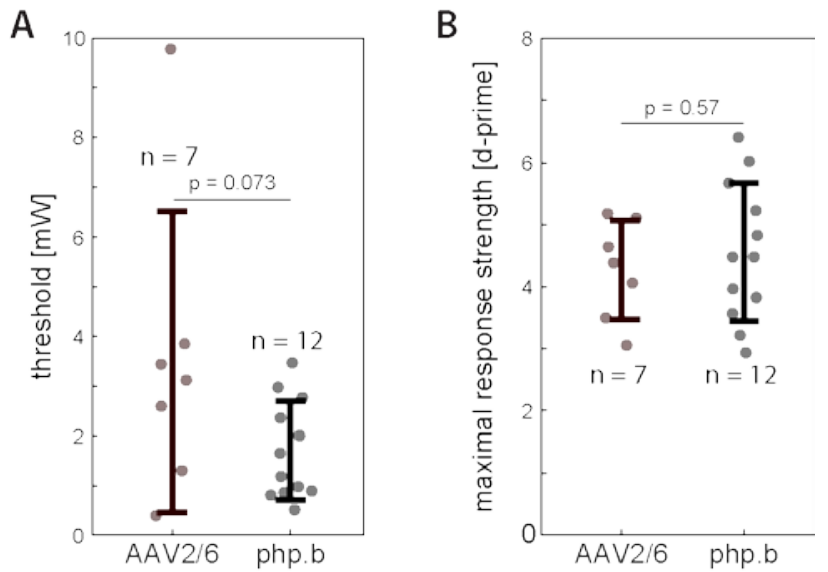

Supplementary Figure 13: Comparison of AAV2/6 and AAV-php.b injected animals. (A) Thresholds $\left(d^{\prime}=1\right)$ of individual animals at the best electrode(two-sample t-test; $n=7($ AAV2/6)/ $n=12$ (php.b)(. (B) Strongest response that could be evoked in each animal (twosample t-test; $n=7(\operatorname{AAV} 2 / 6) / n=12$ (php.b)). Data is depicted as mean \pm s.d.. Source data is provided as a source data file. 

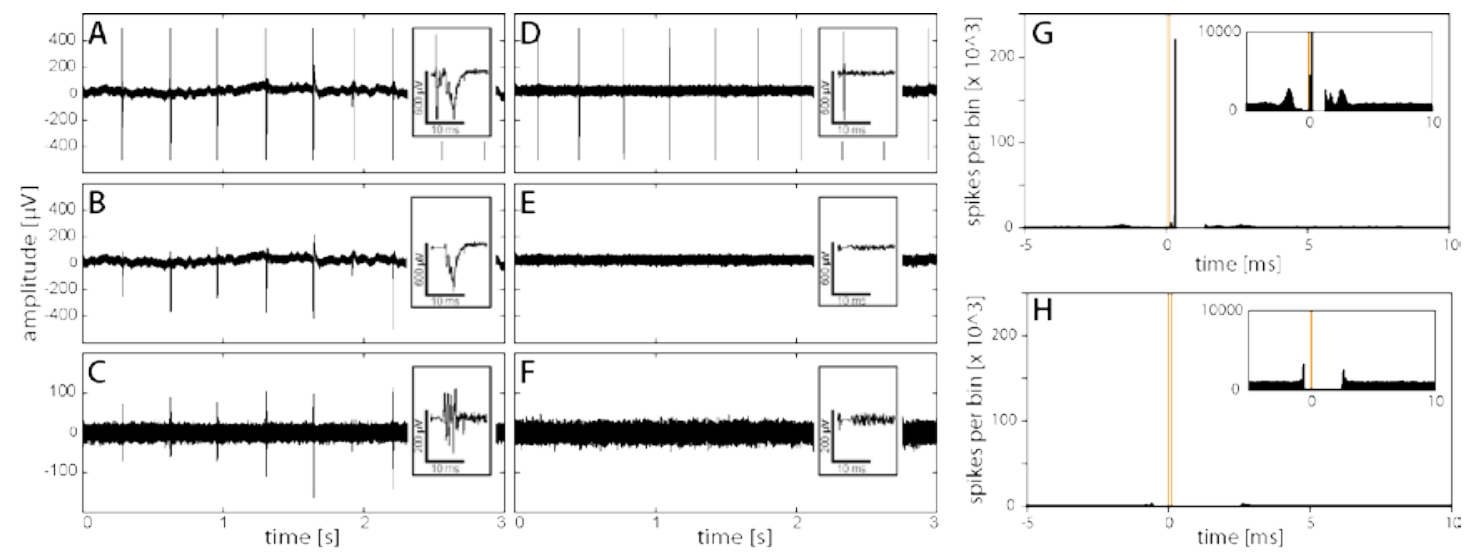

Supplementary Figure 14: Artefact removal of electrical stimulation. (A) "raw" data trace (hardware-filtered from 0.1$9 \mathrm{kHz}$ ) including the electrical artefacts before linear interpolation. (B) The same data trace after linear interpolation of a $3 \mathrm{~ms}$ window around the stimulation artefact. (C) $0.6-6 \mathrm{kHz}$ band-pass filtered data of the trace shown in (A) and (B) in order to extract multi-unit activity. (D-E) Data display as in (A-C), but here measured post-mortem in a sacrificed animal. No waveforms were observed after artefact removal. (G-H) Peri-Stimulus-Time-Histograms of detected waveforms upon electrical stimulation without clipping of the artefact $(\mathrm{G})$ and with clipping of the artefact $(\mathrm{H})$. Spike counts per bin are summed across all animals and stimulation intensities. Orange lines indicate stimulus on- and offset. Insets show a zoom-in of the $y$-axis. 


\section{Chapter III: $\mu$ LED-based optical cochlear implants for spectrally selective activation of the auditory nerve}

Alexander Dieter ${ }^{1,2^{*}}$, Eric Klein ${ }^{3 *}$, Daniel Keppeler ${ }^{1}$, Lukasz Jablonski ${ }^{1,4}$, Tamas Harczos ${ }^{1,4}$, Gerhard Hoch $^{1,4}$, Vladan Rankovic ${ }^{1,4,5}$, Marcus Jeschke ${ }^{1,4,6}$, Patrick Ruther ${ }^{3,7}$, Tobias Moser ${ }^{1,2,4,8}$

${ }^{1}$ Institute for Auditory Neuroscience and InnerEarLab, University Medical Center Göttingen, 37075 Göttingen, Germany

${ }^{2}$ Göttingen Graduate School for Neurosciences and Molecular Biosciences, University of Göttingen, 37075 Göttingen,

\section{Germany}

${ }^{3}$ Department of Microsystems Engineering (IMTEK), University of Freiburg, 79110 Freiburg, Germany

${ }^{4}$ Auditory Neuroscience and Optogenetics Laboratory, German Primate Center, 37077 Göttingen

${ }^{5}$ Restorative Cochlear Genomics Group, Auditory Neuroscience and Optogenetics Laboratory, German Primate Center, 37077 Göttingen

${ }^{6}$ Cognitive Hearing in Primates Group, Auditory Neuroscience and Optogenetics Laboratory, German Primate Center, 37077 Göttingen, Germany

${ }^{7}$ BrainLinks-BrainTools, Cluster of Excellence, University of Freiburg, 79110 Freiburg, Germany

${ }^{8}$ Auditory Neuroscience Group, Max Planck Institute for Experimental Medicine, 37075 Göttingen, Germany

Correspondence: tmoser@gwdg.de, ruther@imtek.de; *equal contribution 


\section{Author contributions:}

A.D., E.K., M.J., P.R. and T.M. designed the study. E.K. and P.R. designed optical cochlear implants. E.K. manufactured optical cochlear implants. L.J. and T.H. designed software for driving optical cochlear implants. G.H. designed hardware for driving optical cochlear implants. M.J. designed hardware and software for multichannel electrophysiological recordings. V.R. produced the virus used in this study. A.D. performed virus injections and electrophysiological recordings. A.D. performed data analysis under the supervision of M.J. and T.M. D.K. performed post-mortem xray reconstructions and initial insertion studies in explanted cochleae to optimize oCl design. A.D. and T.M. prepared the initial draft of the manuscript. All authors edited and finalized the manuscript.

\section{Detailed author contributions of Alexander Dieter (A.D.):}

Conceptual work:

- Contribution to experimental design, initially layouted by Prof. Dr. T. Moser

\section{Experimental work:}

- Intramodiolar virus injections in adult Mongolian gerbils

- Cochlear surgery and placement of cochlear implants

- In vivo multi-electrode electrophysiological recordings from the auditory midbrain

Data analysis:

- Development of Matlab-Code for spike extraction, analysis, statistics, and data display

\section{Data presentation:}

- Manuscript preparation (first draft and editing) under close supervision of Prof. Dr. T. Moser

- Preparation of figures 1, 2, and 3 displayed in the manuscript and figures $1,2,3,4,5,6,7$, and 8 displayed in the supplementary information 


\begin{abstract}
Electrical cochlear implants (eCls) restore hearing and enable speech comprehension to half a million implantees, thereby re-connecting deaf patients to the auditory scene surrounding them. Yet, eCls suffer from limited spectral selectivity, resulting from current spread around each electrode contact and causing poor speech recognition in background noise. Optogenetic stimulation of the auditory nerve might overcome this limitation, as light can be conveniently confined in space. Here, we combined virus-mediated optogenetic manipulation of cochlear neurons and microsystems technology to establish acute multi-channel optical cochlear implant (oCl) stimulation in adult Mongolian gerbils. 16-channel $\mu$ LED-based oCls evoked activation of the auditory pathway with high spectral selectivity and modest power requirements in hearing and deaf gerbils. These results prove the feasibility of $\mu$ LED-based oCls for spectrally selective activation of the auditory nerve.
\end{abstract}




\section{Introduction}

According to the World Health Organization, $6.1 \%$ of the human population suffers from disabling hearing loss, with an economic impact of 750 billion dollar spent on treatment development. Approximately $90 \%$ of the cases suffer from sensorineural hearing loss resulting from dysfunction of the cochlea and/or the auditory nerve. As of today, causal therapies for sensorineural hearing loss do not exist. Hence, the methods of choice for hearing restoration are hearing aids and the electrical cochlear implant $(\mathrm{eCl})$. When hearing loss is profound or complete, eCls are used to bypass dysfunctional or lost sensory hair cells and electrically stimulate spiral ganglion neurons (SGNs). eCls utilize the intrinsic place-frequency-map (known as tonotopy) of the SGNs to provide the patient with spectral information ${ }^{1}$. However, while open speech comprehension is achieved in most of the $500,000 \mathrm{eCl}$ users, there is a major unmet clinical need for improvement of hearing restoration. Current spread in the electro-conductive cochlear fluids limits the spatial - and thus spectral - selectivity of SGN activation by eCls and thus the resolution of electric sound encoding. This ultimately compromises signal perception, especially in noisy environments ${ }^{2-4}$. While efforts are being undertaken towards more spectrally selective electrical SGN activation, e.g. via multipolar stimulation ${ }^{5}$ or direct electrode-neural interfacing ${ }^{6}$, it seems that the spread of excitation remains the bottleneck of the $\mathrm{eCl}$.

Recently it has been suggested that optical stimulation, which - opposed to electric current - can be conveniently confined in space, could activate SGNs with higher spectral selectivity. Thus, optical cochlear implants (oCls) could increase the frequency resolution of artificial sound encoding ${ }^{7,8}$. Two approaches have been proposed for optical activation of SGNs: i) direct infrared neural stimulation ${ }^{7}$ and ii) optogenetics ${ }^{8}$. While infrared stimulation has remained controversial ${ }^{9-}$ ${ }^{11}$, the use of light-gated ion channels (channelrhodopsins, ChRs) for optogenetic SGN stimulation has been reported by at least two laboratories ${ }^{8,12}$. Establishing optogenetic hearing restoration is a challenging, interdisciplinary task ${ }^{13-16}$. Two major objectives must be met: First, optogenetics must render SGNs light sensitive and enable neural coding at good temporal fidelity. Second, multi-channel oCls must allow for spectrally more selective SGN stimulation as eCls. Temporally precise optical SGN control has been established with fast gating ChR variants ${ }^{17-19}$. Furthermore, spatially confined SGN stimulation by individual optical fibers has been shown to elicit neural responses with higher spectral selectivity than electrical stimulation ${ }^{8,20}$. Thus, studies on the biomedical prerequisites to enable optical sound encoding are very promising.

Towards the development of multi-channel optical cochlear implants, oCls have been fabricated both with commercially available LEDs and with gallium-nitride-based, custom-developed thin- 
film LEDs in the micrometer range ( $\mu$ LEDs $)^{21-24}$. While LED-based implants ${ }^{23,24}$ typically carried 1015 LEDs with emitters sizes from 0.22-1 mm, $\mu$ LED-based implants were manufactured with up to 144 emitters of $60 \times 60 \mu \mathrm{m}^{22}$. $\mu$ LEDs of these implants achieve an optical power output of up to 0.82 $\mathrm{mW}$ (corresponding to a power density of $407 \mathrm{~mW} / \mathrm{mm}^{2}$ ), with a maximum temperature increase of $1^{\circ} \mathrm{C}$ (driven at $10 \mathrm{~mA}$ ), and are thus efficient enough to drive most ChRs and safe enough for in vivo application ${ }^{22}$. Peak intensity and light extraction of these $\mu$ LEDs could further be increased (by $95 \%$ and $83 \%$, respectively) by the implementation of conical concentrators and spherical micro-lenses on the emitter surface ${ }^{25}$. However, to our knowledge optogenetic SGN activation in vivo by such implants have not been demonstrated yet.

In this study, we combined viral gene transfer of the ChR-variant CatCh into SGNs of adult Mongolian gerbils with optical stimulation by 16 -channel, $\mu$ LED-based oCls. We demonstrate $\mu L E D$-mediated activation of the spiral ganglion by 32-channel recordings of multi-neuronal clusters in the auditory midbrain in hearing and deaf gerbils. SGN activation was achieved with individual $\mu L E D s$, and the strength of responses increased when recruiting additional emitters. Finally, $\mu$ LED-based optogenetic stimulation achieved increased spectral selectivity as compared to electrical stimulation via clinical-style eCls. This proof of increased frequency selectivity by $\mu L E D-b a s e d$, multi-channel oCls raises hopes that optogenetic hearing restoration might indeed overcome the major limitations of $\mathrm{eCls}$, and one day might increase the quality of artificial sound encoding for deaf patients. 


\section{Results}

Multi-channel optical control of the auditory nerve: To render SGNs light sensitive, we intramodiolarly injected a suspension of adeno-associated virus (AAV-php. b $^{26}$ ) carrying the calcium translocating channelrhodopsin CatCh, fused to eYFP under control of the human synapsin promoter, into the cochlea of adult gerbils $^{18,20}$. Functional expression of CatCh was probed by recordings of auditory brainstem activity upon illumination of the cochlea with a laser-coupled optical fiber earliest four weeks after virus-injection (Suppl. Fig. 1). A subset of animals has been deafened by intracochlear application of kanamycin, which leads to loss of hair cells and hence creates a model of sensorineural hearing loss ${ }^{18}$. Deafening was confirmed by recordings of auditory brainstem activity in response to acoustic clicks. Mean thresholds of click-evoked auditory brainstem responses amounted to $30 \pm 0 \mathrm{~dB}$ SPL before deafening

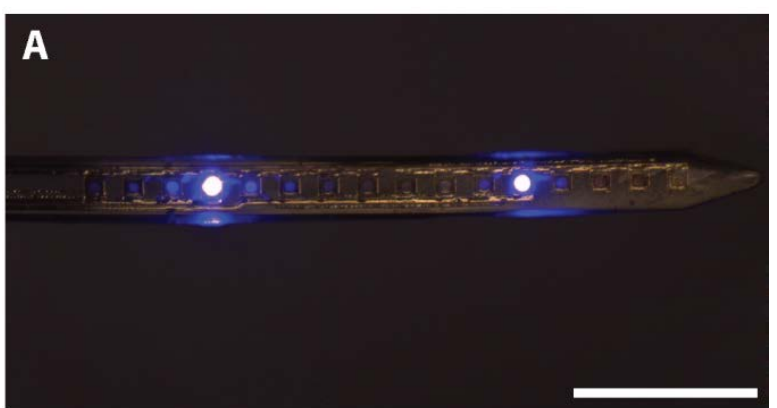

B

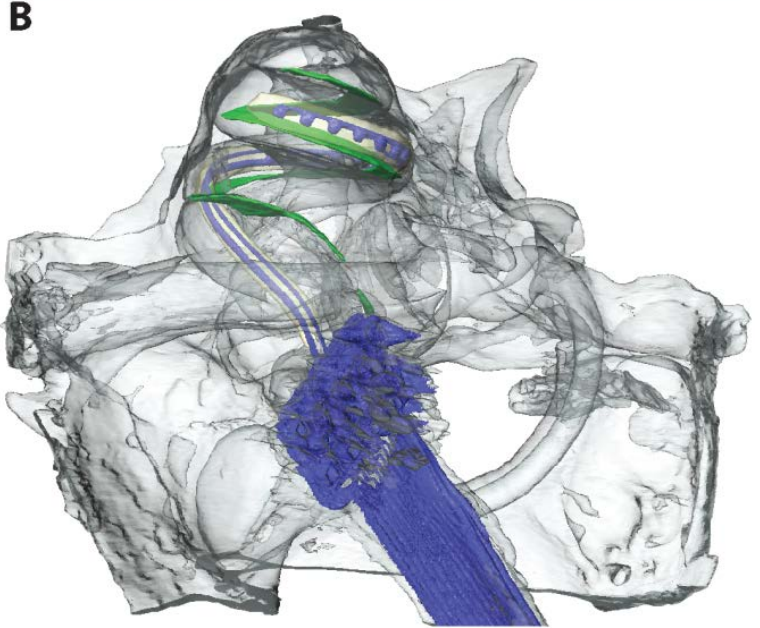

Figure 1: $\mu$ LED-based $\mathrm{oCl}$. (A) Picture of an optical $\mathrm{Cl}$ carrying 16 individually addressable $\mu$ LEDs with a pitch of $100 \mu \mathrm{m}$ on a flexible substrate, $\mu L E D s$ \#5 and \#14 (from the tip) are active. Scale bar: $500 \mu \mathrm{m}$. (B) 3D X-ray tomography reconstruction of a 16-channel optical $\mathrm{Cl}$ implanted in a gerbil cochlea. Cables and $\mu$ LEDs are marked in blue; the basilar membrane is marked in green. $(n=4)$ and no responses were observed with clicks up to $100 \mathrm{~dB}$ SPL 5-10 days after kanamycin application (Suppl. Fig. 2A). Optogenetic activation of the auditory nerve was still possible in deafened animals (Suppl. Fig. 2B). For multichannel optical stimulation, we used oCls housing 16 individually addressable $\mu$ LEDs (60x60 $\mu \mathrm{m})$ with a center-to-center pitch of either 100 or $250 \mu \mathrm{m}$ embedded in biocompatible epoxy material (Fig. $1 \mathrm{~A})^{22}$. The maximum power output mounted to $0.76 \mathrm{~mW}$ for individual $\mu \mathrm{LEDs}$ and $2.94 \mathrm{~mW}$ when all emitters of an oCl were activated (Suppl. Fig. S3). Using a retroauricular approach, the oCls were inserted into the cochlea of isoflurane-anesthetized gerbils via the round window or cochleostomy (Fig. 1B). Electrophysiological recordings of multi-unit activity have been performed 
in the central nucleus of the inferior colliculus (ICC) using linear 32-channel multi-electrode arrays. Since both the spiral ganglion and the ICC are characterized by a remarkably conserved tonotopic organization, the frequency range of SGN activation in the cochlea can be inferred from neural activation in the ICC.

Acoustic calibration: To interpret neural activation in response to oCl stimulation and compare data across animals, electrode positioning along the tonotopic axis of the ICC was physiologically confirmed by acoustic stimulation using pure tones of varying frequency and intensity. Characteristic frequencies were derived for each responsive electrode, and tonotopic slopes were calculated for each animal by a linear fit of characteristic frequencies as a function of recording depth (Suppl. Fig. 4). Median tonotopic slopes amounted to 4.34 octaves/mm ( \pm 0.47 median average deviation, $n=9$ ), which is consistent with previous studies (tonotopic slopes were reported between 4.08 and 4.58 octaves $/ \mathrm{mm})^{20,27,28}$. In deafened animals, the recording probe was stereotactically inserted and the median tonotopic slope of regular hearing animals was assigned since acoustic mapping could not be performed.

ICC activation with oCl stimulation: ICC activation by oCl stimulation of SGNs was evaluated based on peri-stimulus time histograms in response to maximum stimulus parameters (i.e. 16 active $\mu L E D s$ at maximum intensity, $\sim 2.9 \mathrm{~mW}$; Suppl. Fig. 5) and neural responses were found to occur 3.4 $-18.8 \mathrm{~ms}$ after stimulus onset. Since a transient stimulus artefact was observed in some traces at stimulus onset, the time window to quantify potential responses was set to $3-20 \mathrm{~ms}$ in order to avoid the artefact but not discard neural responses. During this time window, multi-unit activity in response to a given stimulus of increasing intensity was sorted in a two-dimensional response matrix according to the corresponding recording site and stimulus intensity. From this matrix, the cumulative discrimination index ( $d$-prime, $d^{\prime}$ ) was calculated based on spike rates in response to increasing stimulation intensities, starting with a zero intensity condition (i.e. no stimulation, baseline $e^{6,7,20}$. The cumulative $d^{\prime}$ quantifies the change in response strength as standard deviations from baseline, i.e. a d' of one is equal to a rise in firing rates by one standard deviation. To estimate the patterns of ICC activation, spatial tuning curves (STCS) were constructed based on iso-contour-lines at integer d'-values (Fig. 2A).

When stimulating SGNs with individual $\mu \mathrm{LEDs}$ of the $\mathrm{oCl}$ at maximum intensity, approximately one third of the $\mu$ LEDs (127/352) evoked neural responses in the ICC (i.e. $d^{\prime}$ values greater or equal to 1 at least at one recording site), and an average $d^{\prime}$ value of 2.1 ( \pm 0.78 SD) was obtained from 
responsive multi-units (fig. 2B). Responses increased when recruiting additional $\mu$ LEDs: Upon maximal stimulation with four neighboring $\mu$ LEDs, approximately $60 \%(55 / 88)$ of $\mu$ LED blocks evoked neural responses in the ICC with an average $d^{\prime}$ value of 2.99 ( $\pm 0.93 \mathrm{SD}$ ), and maximal stimulation with all $16 \mu \mathrm{LEDs}$ on the $\mathrm{oCl}$ achieved average response strengths of 3.29 ( $\pm 0.59 \mathrm{SD}$, at $\sim 2.9 \mathrm{~mW}$; fig. 2B). For comparison, SGN stimulation with a laser-coupled optical fiber delivering light of $\sim 35 \mathrm{~mW}$ evoked average response strengths of 4.36 ( $\pm 0.53 \mathrm{SD}$ ). The strength of neural responses did not differ between hearing and deafened animals $(p=0.11 / 0.44 / 0.83 / 0.89$ for individual/block/all/fiber stimulation, post-hoc corrected two-sample t-test for multiple comparisons).

A

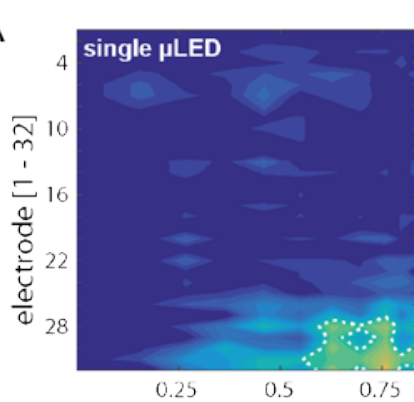

light intensity $[\mathrm{mW}]$

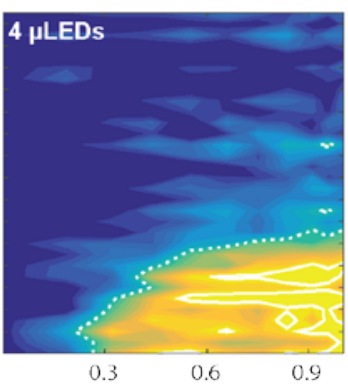

light intensity $[\mathrm{mW}]$

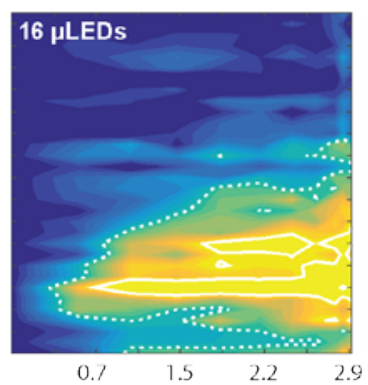

light intensity [ $\mathrm{mW}]$

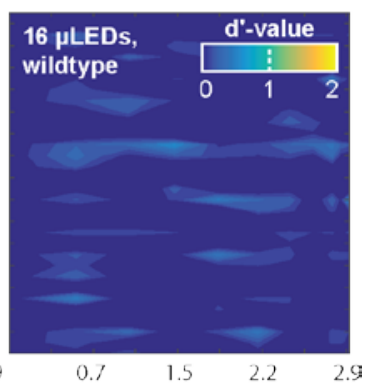

light intensity [mW]
B

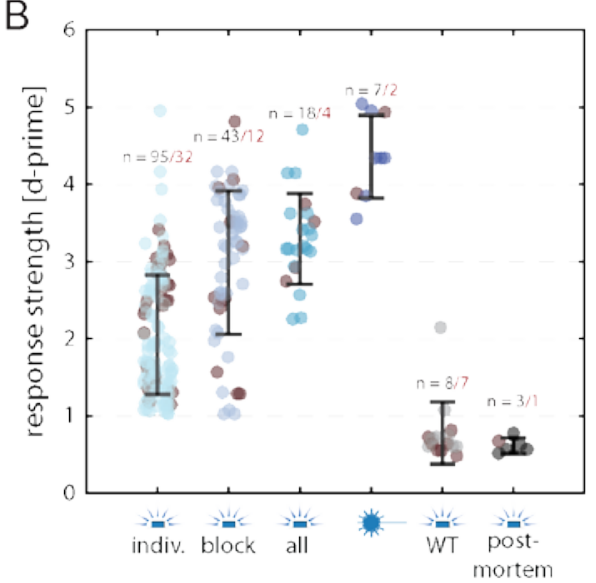

C

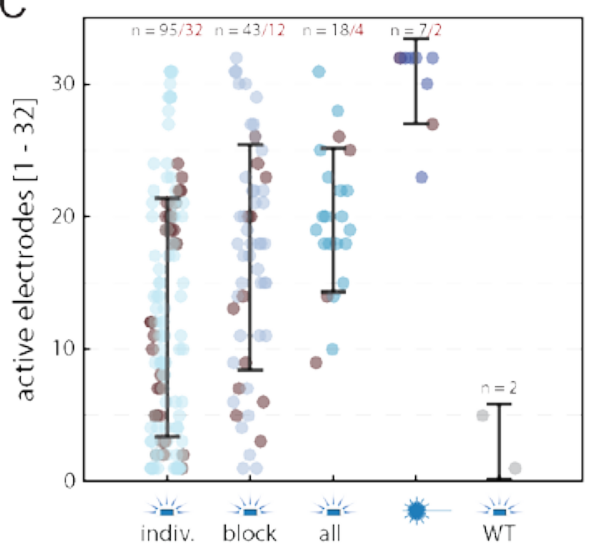

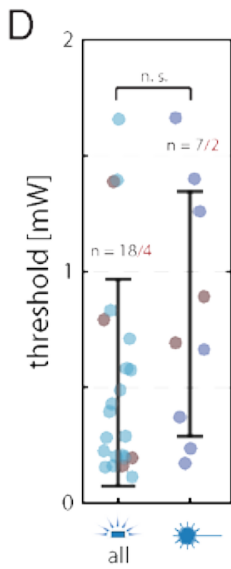

Figure 2: $\mu$ LED-evoked neural responses in the ICC. (A) Exemplary STCs in response to SGN illumination with a single (most basal) $\mu L E D$, a block of four neighboring $\mu$ LEDs and all $\mu L E D s$ in a CatCh-transduced gerbil and with all $\mu L E D s$ in a wildtype gerbil (from left to right). $\mu$ LEDs were spaced by $100 \mu \mathrm{m}$. (B) Maximum strength of ICC responses evoked by oCl stimulation with 1, 4 and 16 active $\mu$ LEDs, and with a laser-coupled optical fiber ( 35 mW) in CatCh-transduced gerbils. oCl stimulation with all $\mu$ LEDs has also been performed in wildtype animals and in sacrificed CatCh-transduced animals. Brown dots indicate data recorded from deafened animals. (C) Active electrodes $\left(d^{\prime}>1\right)$ upon oCl stimulation with 1, 4 and $16 \mu$ LEDs, and with optical fiber stimulation. oCl stimulation with all $\mu$ LEDs has also been performed in wildtype animals. ( $D$ Thresholds ( $d^{\prime}=1$, measured at the best electrode) for ICC activation evoked 16 active $\mu$ LEDs on an oCls and with a laser-coupled optical fiber, respectively ( $p=0.12$, two-sample t-test). 
Weak responses $\left(d^{\prime}=0.68 \pm 0.63\right)$ were also observed in two out of eight measurements from non-injected, hearing control animals (probably due to an opto-acoustic effect), but responses have never been observed in deafened control (no AAV-CatCh injection) or in sacrificed animals. Only 3.0 multi-units (located in dorsal ICC regions (i.e. tonotopically not corresponding to the illuminated regions in the basal cochlea) were responsive to light in hearing control gerbils. Responses had longer latencies (7.5 vs $3.4 \mathrm{~ms}$ ) and shorter duration ( $3.3 \mathrm{vs} 15.8 \mathrm{~ms}$ ) as compared to neural responses in CatCh-injected animals (Suppl. fig. S6). Besides increasing response strengths of individual multi-units, recruitment of additional $\mu$ LEDs also activated broader regions of the ICC: While individual $\mu$ LEDs drove on average $12.4 \pm 9.0$ (out of a maximum of 32 electrodes, limited by the silicon probe design) units per animal, blockwise stimulation evoked activity in $16.9 \pm 8.5$ units, and oCl stimulation with all $\mu$ LEDs recruited $19.7 \pm 5.4$ units (Fig. $2 \mathrm{C}$ ). To demonstrate that these spatially restricted activation patterns originate from confined illumination by the $\mathrm{oCl}$ and not, for example, from spatially limited opsin expression in the spiral ganglion, we employed SGN stimulation with an optical fiber in a less selective manner ${ }^{20}$ : The fiber was placed to broadly illuminate the whole cochlea rather than confining laser at a given cochlear region, and the intensity was set to $\sim 35 \mathrm{~mW}$, the highest possible laser output and by far exceeding the thresholds for CatCh-mediated SGN activation. Stimulating with these settings, multi-unit activity was observed at $30.22 \pm 3.2$ recording sites per animal, indicating that almost all units could potentially be driven by light. Thus, the selective activation of SGNs by more ideally placed oCls ad modest stimulation intensities indeed originates from spatially limited spread of light (Fig. 2C). As for response strengths, also the number of recruited multi-units did not differ between hearing AAV-injected and non-injected animals $(p=0.61 / 0.21 / 0.63 / 0.74$ for individual/block/all/fiber stimulation, two-sample t-test). Thresholds for optical activation were similar for SGN illumination with oCls and optical fibers $(0.52 \pm 0.45 \mathrm{~mW}$ vs. $0.82 \pm 0.53 \mathrm{~mW} ; \mathrm{p}=$ 0.12, two-sample t-test; Fig. 2D). The mean electrical threshold for optical activation amounted to $4.80 \mathrm{~mA}$.

SGN activation with high frequency selectivity: In order to estimate activation patterns in the cochlea, the spatial extent of ICC activity has been quantified by an activity based analysis. From STCs, the iso-contour-line corresponding to a $d^{\prime}$ of one was taken as the threshold of neural activation, as previously done in other studies on electrical and optical auditory nerve stimulation ${ }^{6,7,20,29}$. The recording site with the lowest threshold, i.e. the focus of neural activation, was defined as the best electrode. The spatial extent of neural 
activation, i.e. the spread of cochlear excitation, was defined as the range in the ICC covered by electrodes that recorded optically responsive multi-units at the stimulus intensity at which responses of a fixed $d$ ' value were obtained from the best electrode (Fig. 3A). This activity-based quantification allows for an estimation of neural excitation independent of the nature and absolute intensity of a stimulus, and thus allows for comparison across different modes of stimulation at comparable stimulus strengths. Since neural responses did not differ between hearing and deafened animals, the data of both groups was pooled for subsequent analysis.To demonstrate tonotopic activation of the auditory nerve by oCls, SGNs have been stimulated both with individual $\mu$ LEDs and with blocks of four neighboring $\mu$ LEDs. The $16 \mu$ LEDs on oCls were spaced either by 100 and $250 \mu \mathrm{m}$, and thus covered a cochlear length of 1.5 and $3.75 \mathrm{~mm}$, respectively. Since the length of the scala tympani (where oCls have been inserted) amounts to approximately $11 \mathrm{~mm}$ in gerbils, only 13.6 and $34.1 \%$ of the cochlear length could be covered by the oCls, respectively ${ }^{30}$. To overcome this limitation and demonstrate tonotopic activation over larger parts of the cochlea, some implants have been inserted via a cochleostomy in the middle cochlear turn rather than via the round window. Indeed, SGN illumination at different locations along the cochlear spiral led to spatially confined neural activation of tonotopically corresponding ICC regions (Fig. 3A).

To correct for different locations of $\mathrm{oCl}$ insertion (round window vs cochleostomy), different insertion depths (due to varying implant lengths), and different $\mu$ LED-pitch,

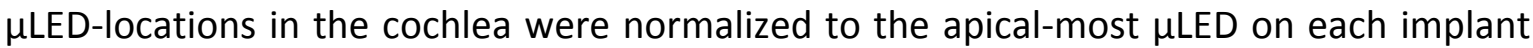
which elicited neuronal responses in the ICC. Hence, the best electrode of each STC has been normalized to the best electrode which was evoked by the apical-most $\mu$ LED in the cochlea. In other words, for each implant and animal, $\mu L E D$-dependent shifts in neural activation have been normalized to the most apical $\mu$ LED in the cochlea and the focus of neural activation evoked by this $\mu$ LED. Upon SGN illumination with different $\mu$ LEDs, a (non-significant) trend for stimulus-location-dependent ICC activation was observed which amounted to 1.92 electrodes (i.e. $96 \mu \mathrm{m}$ ) in the ICC per millimeter stimulus location in the cochlea when stimulating with individual $\mu$ LEDs (Pearson's $r=0.073$ ) and 1.21 


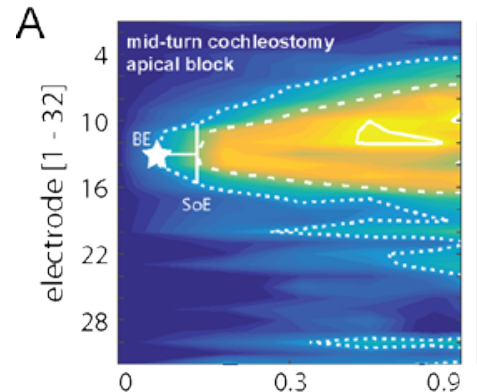

light intensity [mW]

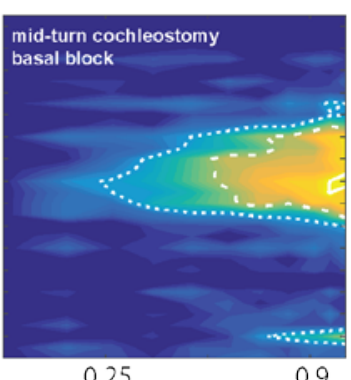

0.25 light intensity [mW]

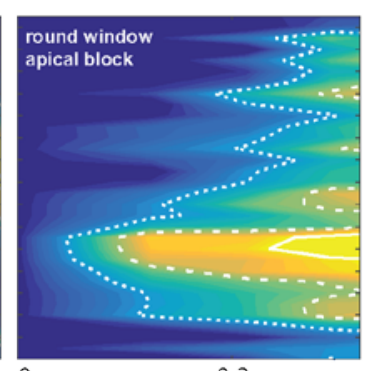

0.3

light intensity [mW]

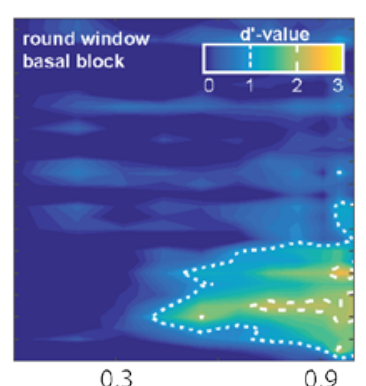

light intensity [mW]
B

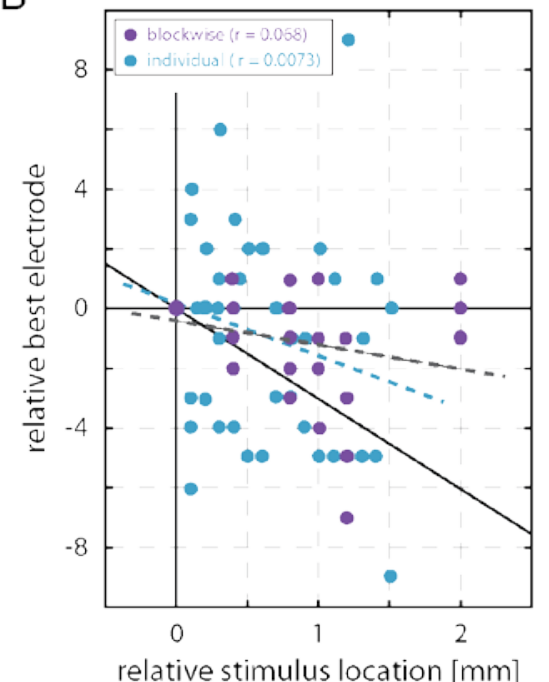

C

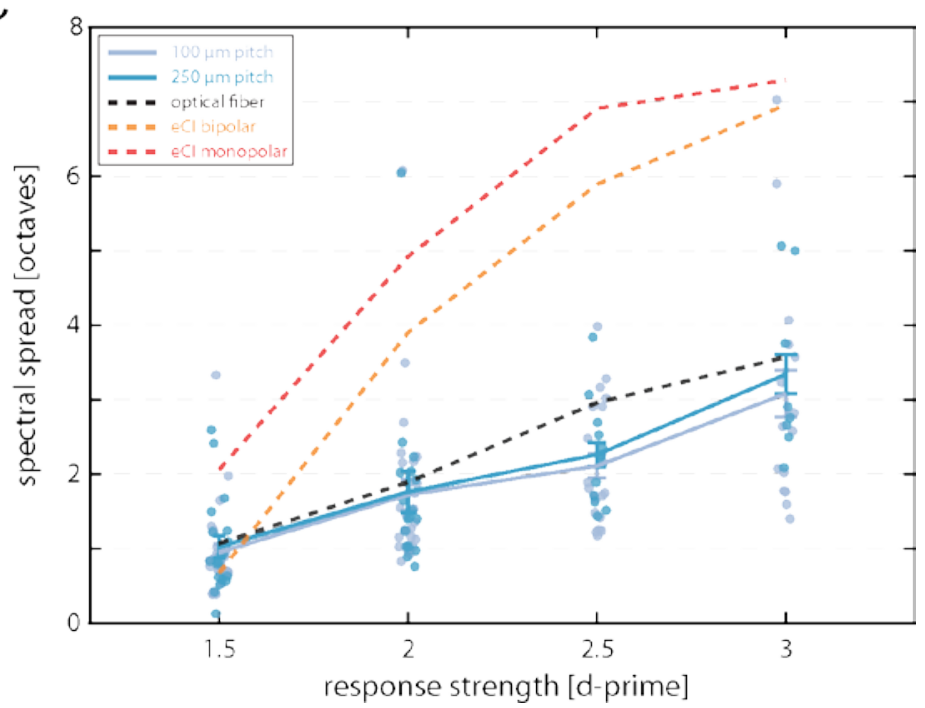

Figure 3: Spectral features of oCl coding. (A) Exemplary STCS in response to SGN stimulation with the four apical- and four basal-most $\mu \mathrm{LEDs}$ of an $\mathrm{OCl}$ inserted via a mid-turn cochleostomy (left, center left) and via the round window (center right, right). Definitions of the best electrode (BE) and spread of excitation (SoE) are indicated in the left panel. (B) Relative best electrode as a function of stimulus location, normalized to the apical-most $\mu$ LED evoking a response. The black line indicates the tonotopic axis as expected based on literature values. (C) Spectral spread of excitation upon SGN stimulation with $\mu$ LED-based oCls (solid lines; mean \pm SEM), laser-coupled optical fibers, as well as mono- and bipolar electrical stimulation with a clinical-style eCl (dashed lines). Data of stimulation via optical fibers and electrical Cls is reprinted from ${ }^{20}$.

Intracochlear spread of excitation (SoE) was estimated at different levels of response strength in the ICC. Since the average strength of neural responses to optical stimulation with individual $\mu$ LEDs did not exceed a d' of two (Fig. 2B), blockwise stimulation with four neighboring $\mu$ LEDs at a time was performed for estimating the spatial extent across a larger range of activation levels. Generally, the spread of neural excitation in the ICC upon intracochlear oCl stimulation increased with growing response strengths at the best electrode (Fig. 3C). The spatial extent of neural excitation was then transferred to spectral 
spread by using the tonotopic slope of each animal (in case of deaf animals, this transformation was done by using the median tonotopic slope of hearing animals). The spread of excitation was similar when stimulating with a $\mu$ LED pitch of $100 \mu \mathrm{m}(0.95 \pm$ $0.12 / 1.72 \pm 0.21 / 2.11 \pm 0.16 / 3.08 \pm 0.31$ octaves at $d^{\prime}$ of $1.5 / 2 / 2.5 / 3$; mean \pm SEM) compared to a pitch of $250 \mu \mathrm{m}(1.02 \pm 0.15 / 1.76 \pm 0.28 / 2.26 \pm 0.16 / 3.34 \pm 0.26$ octaves; $p$ $=0.68 / 0.90 / 0.60 / 0.69$, repeated measures ANOVA and post-hoc pairwise comparison; Fig. 3C). These findings were identical when the spread of ICC activation was not normalized by the tonotopic slope, i.e. is reported as physical space in the ICC (Suppl. fig. 7). 


\section{Discussion}

In this study we have applied multi-channel, $\mu$ LED-based optical cochlear implants to activate the optogenetically modified auditory nerve, successfully combining the biomedical and optoelectronic work towards optogenetic hearing restoration. We demonstrated in vivo functionality and high spectral selectivity of optogenetic SGN stimulation by $\mu$ LED-based oCls in a rodent model of cochlear optogenetics.

oCl implantation: For two of the implants, $\mathrm{x}$-ray tomograms have been reconstructed. Interestingly, light-emitting surfaces of the $\mu$ LEDs were facing Rosenthal's canal in both cases and hence achieve ideal illumination of the spiral ganglion - a fact of critical importance for the functionality of optogenetic hearing restoration. However, in both cases, the implant pierced through the basilar membrane at the base of the cochlea, crossing from scala tympani to scala media (Fig. 1B, Suppl. Fig. 8). This might be due to the limited flexibility of the implant: Both the planar structure of the $\mu \mathrm{LEDs}$ and the parallel power lines to address $\mu \mathrm{LEDs}$ limit oCI flexibility to the plane of emitter surfaces. We speculate that the current oCls were not flexible enough to be maintained in the gerbil scala tympani, respecting the basilar membrane. To overcome this problem, oCls could be implanted via a basal cochleostomy which optimizes the insertion angle in future studies.

oCl-mediated neural activation: The average threshold of neural activation in the ICC upon oCI stimulation of SGNs was found to be $0.52 \mathrm{~mW}$ (4.8 $\mathrm{mA}$ driving current). Thresholds tended to be lower (even though the difference was not significant, $p=0.12$ ) for stimulation by $\mu$ LEDs as compared to fiber-based stimulation, which amounted to $0.82 \mathrm{~mW}$. In a previous study using the same settings, the average threshold of fiber-based stimulation was reported to be $1.7 \mathrm{~mW}^{20}$. As expected and demonstrated by Monte Carlo modelling, optical power needed for SGN excitation is lower when reducing the emitter deistance from the spiral ganlgion ${ }^{20}$. Since the intracochlear $\mu L E D s$ used in this study are located closer to the neural target tissue as optical fibers positioned at the round window, the trend of lower thresholds most likely results from improved emitter placement. The maximum $d^{\prime}$ values of neural responses in the ICC amouned to a $d^{\prime}$ of 2.1, 2.99 and 3.29 for oCl stimulation via 1,4 , and 16 active $\mu$ LEDs, respectively. This compares to a $d^{\prime}$ of 4.36 upon fiber-based stimulation in the same animals and a maximum $d^{\prime}$ of 4.4 reported in a previous study, when driving SGNs with an optical power of up to $30 \mathrm{~mW}^{20}$. $\mu \mathrm{LED}$ stimulation 
reached $\sim 75 \%$ of the response strength evoked by optical fibers, with $90 \%$ less optical power $(2.9$ $\mathrm{mW}$ vs $30 \mathrm{~mW})$.

Spectral selectivity of cochlear optogenetics: The average spatial spread of excitation in the ICC upon SGN stimulation with blocks of four neighboring $\mu$ LEDs with a pitch of $100 \mu \mathrm{m}$ amounted to $0.95 / 1.72 / 2.11 / 3.08$ octaves at a $d^{\prime}$ of $1.5 / 2 / 2.5 / 3$ at the $B E$, respectively. A previous study reported a spatial spread of 1.07/1.89/2.96/3.57 octaves upon confined fiber-based optogenetic stimulation, supporting our finding of spectrally precise neural activation of the auditory nerve ${ }^{20}$. While it might seem surprising that the SoE with a block of $4 \mu$ LEDs, i.e. Lambertian emitters, is comparable to that of a $200 \mu \mathrm{m}$ optical fiber, we suspect that this reflects the closer proximity of $\mu L E D s$ to the target tissue. The spread of excitation upon mono- and bipolar stimulation in the same study amounted to 2.06/4.92/6.91/7.29 and 0.67/3.90/5.89/6.96 octaves ${ }^{20}$. Thus, oClmediated activation of the auditory system is feasible with higher spectral selectivity then when using clinical-style eCls, where even bipolar stimulation was less selective for all, but near threshold intensities. Since we were only able to assess the spatial selectivity upon SGN stimulation with groups of four $\mu$ LEDs, we expect that the spatial selectivity of optogenetic SGN stimulation can be further improved by increased light efficacies of $\mu$ LEDs, the implementation of micro-lenses, or the implementation of opsins with a higher light sensitivity, which would allow for robust SGN stimulation with individual $\mu \mathrm{LEDs}^{25}$.

Non-optogenetic neural activation: Upon oCl stimulation in non-injected wildtype gerbils, weak neural responses were observed with 2 out of 8 implants. These responses differed from neural responses in CatCh-transduced animals (Suppl. Fig. 3). We suggest that an opto-acoustic effect might have evoked these responses ${ }^{11}$, as the longer latency might result from the delay of synaptic transmission between the inner hair cell and the SGN, and responses could not evoked in wildtype animals without hair cells. Furthermore, these responses vanished after some time and were not stable over the course of the experiment, most likely due to degradation of the endocochlear potential upon oCl implantation. In contrast, optically evoked responses in CatChtransduced animals were highly stable over the whole experiments. However, even if there was minor contribution to neural excitation by an opto-acoustic effect in the case of CatCh-transduced gerbils (which is unlikely given the comparable patterns of activation in regular hearing and deafened CatCh-injected animals), we have rather over- than underestimated the spread of excitation in this study, since such combined SGN excitation was most likely broader than for exclusive optogenetic stimulation. 
Limitations: Obviously, this study is only contributing one step in the effort to develop optical cochlear implants. Since individual $\mu L E D s$ have a limited power output, neural responses in the IC could not be evoked with all individual $\mu L E D s$ and evoked responses were far away from saturation (Fig. 2B, individual $\mu$ LEDs vs optical fiber). Hence, one major objective of future $\mu$ LED development is increased power output, which might be achieved by the implementation of micro-lenses. Besides the power efficacy, also the number of optical emitters and their pitch should be optimized for the model system used in future experiments. oCls in this study housed $16 \mu$ LEDs with a maximum pitch of $250 \mu \mathrm{m}$, covering at most $3.75 \mathrm{~mm}$, i.e. one third, of the gerbil cochlear length. To optimally access the tonotopic axis of the auditory nerve with high resolution, the number of emitters - ideally spaced by a pitch of $100 \mu \mathrm{m}$ - should be increased, in order to cover larger regions of the cochlea. Furthermore, the depth of implantation in this study was mainly limited by the implant length, which should also be increased for future experiments, especially when considering studies in other animal models such as the common marmoset, which have larger cochleae as compared to rodents. A tapered oCl tip might further increase the implantation depth in order to maximize the access to the tonotopic axis of the cochlea. Finally, biocompatibility and long-term stability of optical cochlear implants need to be evaluated in longitudinal studies upon chronic implantation, ideally by performing behavioral studies involving more complex stimulation patterns in order to determine the perceptual frequency resolution limit of optical sound encoding. 


\section{Methods}

Animals: Data was obtained from 13 Mongolian gerbils (Meriones unguiculatus) between 6-14 months of age. For each surgical procedure, gerbils were placed on a heating pad and anesthetized with Isoflurane (induction: $4 \%$ at $1 \mathrm{l} / \mathrm{min}$; maintenance: $1-2 \%$ at $0.4 \mathrm{l} / \mathrm{min}$ ). Anesthetic depth was monitored by the absence of reflexes and adjusted if necessary. Analgesia was achieved by injections of Buprenorphine $(0.1 \mathrm{mg} / \mathrm{kg}$ BW s.c.). All experiments were performed in compliance with the German national animal care guidelines and approved by the animal welfare office of the state Lower Saxony, Germany (LAVES), and the local animal welfare committee of the University Medical Center Göttingen.

Gene transfer to SGNs: Virus suspension of the recently engineered AAV-variant php.b carrying plasmids that code for the calcium permeable Channelrhodopsin-2-variant CatCh, linked to the reporter eYFP, under control of the human synapsin promoter, has been injected into the spiral ganglion of adult Mongolian gerbils ( $>3$ months of age) as described before $\left(6.99 \times 10^{12} \mathrm{GC} / \mathrm{ml}\right)^{18,20,26,31}$. In summary, access to the cochlea was achieved via a retroauricular approach, and a small hole was drilled into the basal modiolus with a dental file to access the spiral ganglion ${ }^{18,20,32}$. After intramodiolar pressure injections of 2-3 $\mu \mathrm{l}$ virus suspension via micropipettes, muscles and connective tissue were repositioned to close the surgical site and the skin was sutured. Animals recovered for at least four weeks after surgery before electrophysiological measurements were performed.

Deafening: Animals were deafened by bilateral, intracochlear injections of Kanamycin solution ( $3 \mu \mathrm{l}, 100 \mathrm{mg} / \mathrm{ml}$, Kanamysel, Selectavet) as described before ${ }^{18}$. Briefly, the cochlea was accessed by the retroauricular approach described for virus injections, Kanamycin solution was injected via the round window membrane, and a kanamycinsoaked gelatin sponge was placed in the round window niche before closing the surgical site with connective tissue and suturing the skin.

Implant fabrication: Multi-channel optical cochlear implants were fabricated as described elsewhere ${ }^{22}$. Briefly, 16 Gallium-Nitride-based $\mu$ LEDs of $60 \times 60 \mu \mathrm{m}$ with a pitch of 100 or 
$250 \mu \mathrm{m}$ were integrated in a flexible, highly transparent, and biocompatible epoxy material. $\mu$ LEDs are individually addressable via a $4 \times 4$ matrix interconnection with $4 \mathrm{n}$ and 4 p-contacts. Light emission and the $\mu$ LED $n$-side contact are realized via a circular aperture (50 $\mu \mathrm{m}$ diameter) in the $n$-metallization on top of the emitting surface. A waferlevel process was established to achieve a high process yield: In summary, $\mu$ LEDs were fabricated from epitaxial grown Gallium Nitride on sapphire substrates. Furthermore, the contacts on the $\mathrm{p}$-side were realized by a high reflective nickel, gold, silver contact and laterally defined by chlorine-based plasma etching. An epoxy-resin-based polymer substrate was then deposited on a sapphire wafer equipped with a sacrificial aluminum layer (to allow for peel-off from the wafer) via a spin-coating process. Subsequently, the $\mu L E D$-wafer was bonded on the polymer-wafer via an Indium-gold inter-diffusion bonding process, and the $\mu$ LED sapphire wafer was released by laser-lift-off. Gold-based ncontacts were then metallized on the $\mu$ LED surface and an additional layer of epoxy-resin was applied for passivation. Finally, the polymer stack was trenched down to the wafer by oxygen plasma to separate the individual probes. Probes were then released by electrochemical dissolution of the sacrificial aluminum layer below.

Auditory brainstem recordings (ABRs): Acoustic or optogenetic stimulation of the auditory pathway was verified by recordings of compound activity from the auditory nerve and (see next section for details). Evoked potentials were recorded via subdermal, low impedance needle electrodes at the vertex and the mastoid bone, amplified with a custom made amplifier and stored on a hard drive at a sampling rate of $50 \mathrm{kHz}$ for offline analysis. A third needle placed at the back of the animals served as an active shielding electrode.

Auditory midbrain recordings: Activity of multi-neuronal clusters (multi-unit activity, MUA) was recorded with a linear 32 -channel silicon probe $\left(177 \mu \mathrm{m}^{2}\right.$ surface, $1-3 \mathrm{M} \Omega$ impedance, $50 \mu \mathrm{m}$ pitch; Neuronexus, Ann Arbor, US) from the central nucleus of the inferior colliculus (ICC) and was described in detail before ${ }^{20}$. Briefly, the ICC was stereotactically accessed via a craniotomy contralateral to the stimulated ear, and the silicon probe was inserted $\sim 2 \mathrm{~mm}$ lateral and $\sim 0.5 \mathrm{~mm}$ caudal to lambda to an initial 
depth of $\sim 3.3 \mathrm{~mm}$ (measured from the surface of visual cortex, which partially covers the auditory midbrain in gerbils ${ }^{33}$ ) using a micromanipulator (LN Junior 4 RE, Luigs \& Neumann; Ratingen, Germany). After initial mapping of MUA with acoustic tones, the silicon probe was re-positioned as needed in order to optimally access the tonotopic axis of the $\mathrm{ICC}^{27,34}$. An epidural low-impedance metal wire $(<1 \Omega)$ served as a reference electrode on the contralateral hemisphere. Using the Digital Lynx 4s System (Neuralynx; Dublin, Ireland), multi-unit responses were amplified, filtered $(0.1-9,000 \mathrm{~Hz})$, digitized $(32$ $\mathrm{kHz}$ sampling rate) and stored on a hard-drive for offline analysis. Once the preparation was done, stimuli were designed with custom-written Matlab-scripts (The MathWorks, Inc.; Natick, US) and generated with a custom-made system based on NI-DAQ-Cards (NI PCl-6229; National Instruments; Austin, US). Near field acoustic stimulation was performed with a loudspeaker (Scanspeak Ultrasound; Avisoft Bioacoustics, Glienicke, Germany) calibrated with a 0.25-inch microphone (4039; Brüel \& Kjaer GmbH, Naerum, Denmark), pre-amplifier (2670) and measurement amplifier (2610) positioned $30 \mathrm{~cm}$ in front of the animal's head. For optical SGN stimulation, access to the inner ear was realized with the retroauricular approach described for virus injections ${ }^{18,20,32}$. $\mu$ LEDs were inserted into the scala tympani via the round window, with $\mu$ LEDs facing the center of the cochlea. $\mu$ LEDs were then driven individually or blockwise with current pulses of $1 \mathrm{~ms}$ between 0-10 mA delivered from a custom-made current source. For fiber-based stimulation, a laser-coupled (473 nm, 100 mW DPSS; Changchun New Industry Optoelectronics) optical fiber (200 $\mu$ m diameter, 0.39 NA; Thorlabs, Dachau, Germany) was inserted to the cochlea via the round window and directed towards the apex to broadly illuminate the cochlea.

Data analysis: All data analysis was performed with custom-written Matlab-scripts. Time stamps of multi-units were extracted as peaks exceeding a threshold (median plus three median absolute deviations) from filtered data traces $\left(0.6-6 \mathrm{kHz}, 4^{\text {th }}\right.$ order Butterworthfilter). After each time stamp, an artificial refractory period of $1 \mathrm{~ms}$ was implemented to avoid overestimating the spike rates. Frequency tuning of multi-units was assessed by presenting $100 \mathrm{~ms}$ pure tones (5 ms sine ramps) of frequencies between $0.5-32 \mathrm{kHz}$ (quarter octave steps) and varying sound pressure level in a pseudo-random order at a 
repetition rate of $4 \mathrm{~Hz}$. Frequency response areas were constructed from 20-30 repetitions of each frequency-intensity combination, and the frequency that evoked neural activity at the lowest intensity was defined as the characteristic frequency ${ }^{35,36}$. For optical stimulation, multi-unit responses occurring 3-20 ms after stimulus onset (1 ms light pulses of varying intensity) were sorted into a response matrix according to stimulus intensity and recording site. Spatial tuning curves have then been constructed based on the cumulative discrimination index of response rates in response to increasing stimulus intensity as described previously, ${ }^{6,20,29}$. Iso-contour lines were subsequently calculated by the contour function provided by Matlab. The iso-contour line at a d' of 1 was then defined as the threshold for neural activation, and the recording site with the lowest threshold was defined as the best electrode. The spread of neural excitation, i.e. the distance spanned by electrodes that recorded a $d^{\prime}$ greater or equal to 1 , was then calculated at the stimulus intensity that evoked a $d^{\prime}$ of $1.5 / 2 / 2.5$ or 3 at the best electrode. If electrodes which recorded responsive multi-units were separated by electrodes with multi-units below threshold $\left(d^{\prime}=1\right)$, the distance between the dorsal- and ventral-most electrodes with a d' greater or equal to 1 were used to calculate the spread of excitation in order to avoid underestimation.

X-ray tomography: After acquisition of electrophysiological data, some oCls have been mounted into the scala tympani with dental cement. Positioning of the implant and direction of the $\mu$ LEDs light emitting surface was assessed with $x$-ray tomography as described previously ${ }^{18,37}$. Data acquisition was achieved with a customized imaging system for cone-beam in-line phase contrast tomography based on a liquid metal $x$-ray source and a LuAG-scintillator-based detector with a pixel size of $6.5 \mu \mathrm{m}$, and a fast Fourier-based phase reconstruction procedure. Segmentation and visualization of reconstructed structures were achieved with the Avizo 3D 9 software and cochlear structures as well as oCl components were traced semi-automatically. 


\section{References}

1. Zeng, F.-G., Rebscher, S., Harrison, W. V., Sun, X. \& Feng, H. Cochlear Implants:System Design, Integration and Evaluation. IEEE Rev. Biomed. Eng. 1, 115-142 (2008).

2. Middlebrooks, J. C., Bierer, J. A. \& Snyder, R. L. Cochlear implants: the view from the brain. Curr. Opin. Neurobiol. 15, 488-493 (2005).

3. Kral, A., Hartmann, R., Mortazavi, D. \& Klinke, R. Spatial resolution of cochlear implants: the electrical field and excitation of auditory afferents. Hear. Res. 121, 11-28 (1998).

4. Friesen, L. M., Shannon, R. V., Baskent, D. \& Wang, X. Speech recognition in noise as a function of the number of spectral channels: Comparison of acoustic hearing and cochlear implants. J. Acoust. Soc. Am. 110, 1150 (2001).

5. Berenstein, C. K., Mens, L. H. M., Mulder, J. J. S. \& Vanpoucke, F. J. Current Steering and Current Focusing in Cochlear Implants: Comparison of Monopolar, Tripolar, and Virtual Channel Electrode Configurations: Ear Hear. 29, 250-260 (2008).

6. Middlebrooks, J. C. \& Snyder, R. L. Auditory prosthesis with a penetrating nerve array. J. Assoc. Res. Otolaryngol. JARO 8, 258-279 (2007).

7. Richter, C.-P. et al. Spread of cochlear excitation during stimulation with pulsed infrared radiation: inferior colliculus measurements. J. Neural Eng. 8, 056006 (2011).

8. Hernandez, V. H. et al. Optogenetic stimulation of the auditory pathway. J. Clin. Invest. 124, 1114-1129 (2014).

9. Verma, R. U. et al. Auditory responses to electric and infrared neural stimulation of the rat cochlear nucleus. Hear. Res. 310, 69-75 (2014).

10. Thompson, A. C. et al. Infrared neural stimulation fails to evoke neural activity in the deaf guinea pig cochlea. Hear. Res. 324, 46-53 (2015).

11. Baumhoff, P., Kallweit, N. \& Kral, A. Intracochlear near infrared stimulation: Feasibility of optoacoustic stimulation in vivo. Hear. Res. 371, 40-52 (2019).

12. Duarte, M. J. et al. Ancestral Adeno-Associated Virus Vector Delivery of Opsins to Spiral Ganglion Neurons: Implications for Optogenetic Cochlear Implants. Mol. Ther. (2018). doi:10.1016/j.ymthe.2018.05.023

13. Moser, T. Optogenetic stimulation of the auditory pathway for research and future prosthetics. Curr. Opin. Neurobiol. 34, 29-36 (2015).

14. Richardson, R. T., Thompson, A. C., Wise, A. K. \& Needham, K. Challenges for the application of optical stimulation in the cochlea for the study and treatment of hearing loss. Expert Opin. Biol. Ther. 17, 213-223 (2017). 
15. Weiss, R. S., Voss, A. \& Hemmert, W. Optogenetic stimulation of the cochlea-A review of mechanisms, measurements, and first models. Netw. Bristol Engl. 27, 212-236 (2016).

16. Dombrowski, T., Rankovic, V. \& Moser, T. Toward the Optical Cochlear Implant. Cold Spring Harb. Perspect. Med. 9, (2019).

17. Mager, T. et al. High frequency neural spiking and auditory signaling by ultrafast redshifted optogenetics. Nat. Commun. 9, 1750 (2018).

18. Wrobel, C. et al. Optogenetic stimulation of cochlear neurons activates the auditory pathway and restores auditory-driven behavior in deaf adult gerbils. Sci. Transl. Med. 10, eaao0540 (2018).

19. Keppeler, D., Merino, C. \& Lopez, D. Ultrafast optogenetic stimulation of the auditory pathway by targeting-optimized Chronos. EMBO J. (2018).

20. Dieter, A., Duque-Afonso, C. J., Rankovic, V., Jeschke, M. \& Moser, T. Near physiological spectral selectivity of cochlear optogenetics. Nat. Commun. 10, 1962 (2019).

21. Goßler, C. et al. GaN-based micro-LED arrays on flexible substrates for optical cochlear implants. J. Phys. Appl. Phys. 47, 205401 (2014).

22. Klein, E., Gossler, C., Paul, O. \& Ruther, P. High-Density $\mu$ LED-Based Optical Cochlear Implant With Improved Thermomechanical Behavior. Front. Neurosci. 12, (2018).

23. Schwaerzle, M., Nehlich, J., Ayub, S., Paul, O. \& Ruther, P. Led-based optical cochlear implant on highly flexible triple layer polyimide substrates. in 2016 IEEE 29th International Conference on Micro Electro Mechanical Systems (MEMS) 395-398 (IEEE, 2016). doi:10.1109/MEMSYS.2016.7421644

24. $\mathrm{Xu}, \mathrm{Y}$. et al. Multichannel optrodes for photonic stimulation. Neurophotonics 5, 045002 (2018).

25. Klein, E., Kaku, Y., Paul, O. \& Ruther, P. Flexible $\mu$ LED-based optogenetic tool with integrated $\mu$-lens array and conical concentrators providing light extraction improvements above 80\%. IEEE MEMS 632-635 (2019).

26. Deverman, B. E. et al. Cre-dependent selection yields AAV variants for widespread gene transfer to the adult brain. Nat. Biotechnol. 34, 204-209 (2016).

27. Schnupp, J. W. H., Garcia-Lazaro, J. A. \& Lesica, N. A. Periodotopy in the gerbil inferior colliculus: local clustering rather than a gradient map. Front. Neural Circuits 9, 37 (2015).

28. Harris, D. M., Shannon, R. V., Snyder, R. \& Carney, E. Multi-unit mapping of acoustic stimuli in gerbil inferior colliculus. Hear. Res. 108, 145-156 (1997). 
29. Snyder, R. L., Bierer, J. A. \& Middlebrooks, J. C. Topographic spread of inferior colliculus activation in response to acoustic and intracochlear electric stimulation. J. Assoc. Res. Otolaryngol. JARO 5, 305-322 (2004).

30. Dong, W. \& Olson, E. S. In vivo impedance of the gerbil cochlear partition at auditory frequencies. Biophys. J. 97, 1233-1243 (2009).

31. Kleinlogel, S. et al. Ultra light-sensitive and fast neuronal activation with the $\mathrm{Ca}^{2}+-$ permeable channelrhodopsin CatCh. Nat. Neurosci. 14, 513-518 (2011).

32. Chen, W. et al. Restoration of auditory evoked responses by human ES-cell-derived otic progenitors. Nature 490, 278-282 (2012).

33. Cant, N. B. \& Benson, C. G. An atlas of the inferior colliculus of the gerbil in three dimensions. Hear. Res. 206, 12-27 (2005).

34. Ryan, A. F., Woolf, N. K. \& Sharp, F. R. Tonotopic organization in the central auditory pathway of the Mongolian gerbil: a 2-deoxyglucose study. J. Comp. Neurol. 207, 369-380 (1982).

35. Kiang, N. Y. S., Liberman, M. C. \& Baer, T. Tuning curves of auditory-nerve fibers. J. Acoust. Soc. Am. 61, S27-S27 (1977).

36. Egorova, M., Ehret, G., Vartanian, I. \& Esser, K. H. Frequency response areas of neurons in the mouse inferior colliculus. I. Threshold and tuning characteristics. Exp. Brain Res. 140, 145-161 (2001).

37. Bartels, M., Hernandez, V. H., Krenkel, M., Moser, T. \& Salditt, T. Phase contrast tomography of the mouse cochlea at microfocus x-ray sources. Appl. Phys. Lett. 103, 083703 (2013). 
Acknowledgments: The authors are very grateful to Daniela Gerke for expert help with virus preparation. We thank Ben Deverman and Viviana Gradinaru for providing the PHP.B construct used in this study. We thank Peter Wenig and Daniel Weihmüller for expert technical support on electronics. The work was funded by the European Research Council (ERC) under the European Union's Horizon 2020 research and innovation program (grant agreement no. 670759- advanced grant "OptoHear") to T.M. and the grant OpticalCl of the German Ministry of Research and Education (no. 13N13729) to P.R. and T.M. A.D. is a fellow of the German Academic Scholarship Foundation

Author contributions: A.D., E.K., M.J., P.R. and T.M. designed the study. E.K. and P.R. designed optical cochlear implants. E.K. manufactured optical cochlear implants. L.J. and T.H. designed software for driving optical cochlear implants. G.H. designed hardware for driving optical cochlear implants. M.J. designed hardware and software for multichannel electrophysiological recordings. V.R. produced the virus used in this study. A.D. performed virus injections and electrophysiological recordings. A.D. performed data analysis under the supervision of M.J. and T.M. D.K. performed post-mortem $x$-ray reconstructions and initial insertion studies in explanted cochleae to optimize oCl design. A.D. and T.M. prepared the initial draft of the manuscript. All authors edited and finalized the manuscript.

Competing interests: TM and DK are co-founders of the OptoGenTech company. 


\section{Supplementary Information}

Supplementary Figures:

Supplementary figure 1: Optically evoked auditory brainstem activity.

Supplementary figure 2: Deafening by intracochlear kanamycin application.

Supplementary figure 3: Optical power of $\mu$ LED-based oCls.

Supplementary figure 4: Tonotopy in the auditory midbrain.

Supplementary figure 5: Response window of oCl evoked neural responses.

Supplementary figure 6: Neural responses in wildtype animals

Supplementary figure 7: Spatial spread of excitation

Supplementary figure 8: Tomogram of the oCl-implanted cochlea. 


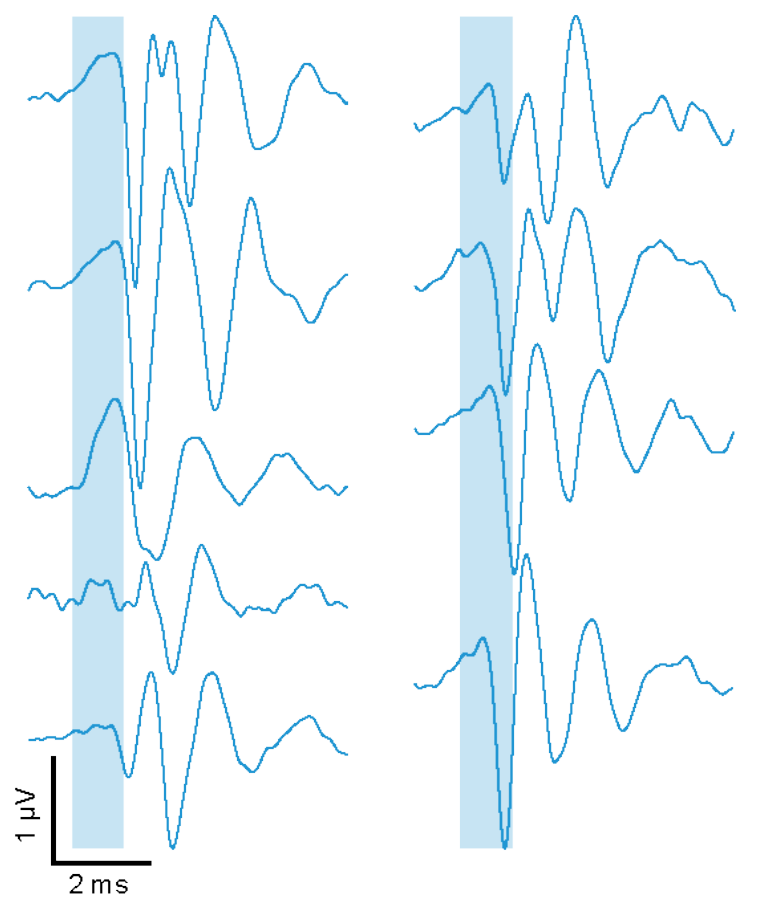

Supplementary Figure 1: Optically evoked auditory brainstem responses. Functional opsin expression was verified by recordings of optically evoked activity in the auditory nerve and brainstem at the start of each experiment before stimulating SGNs with oCls for IC recordings. Optical stimulation occurred with laser pulses of $\sim 35 \mathrm{~mW}$ intensity and $1 \mathrm{~ms}$ pulse duration at a stimulation rate of $10 \mathrm{~Hz}$. Each trace represents the average of 1000 stimulus presentations in one animal. 

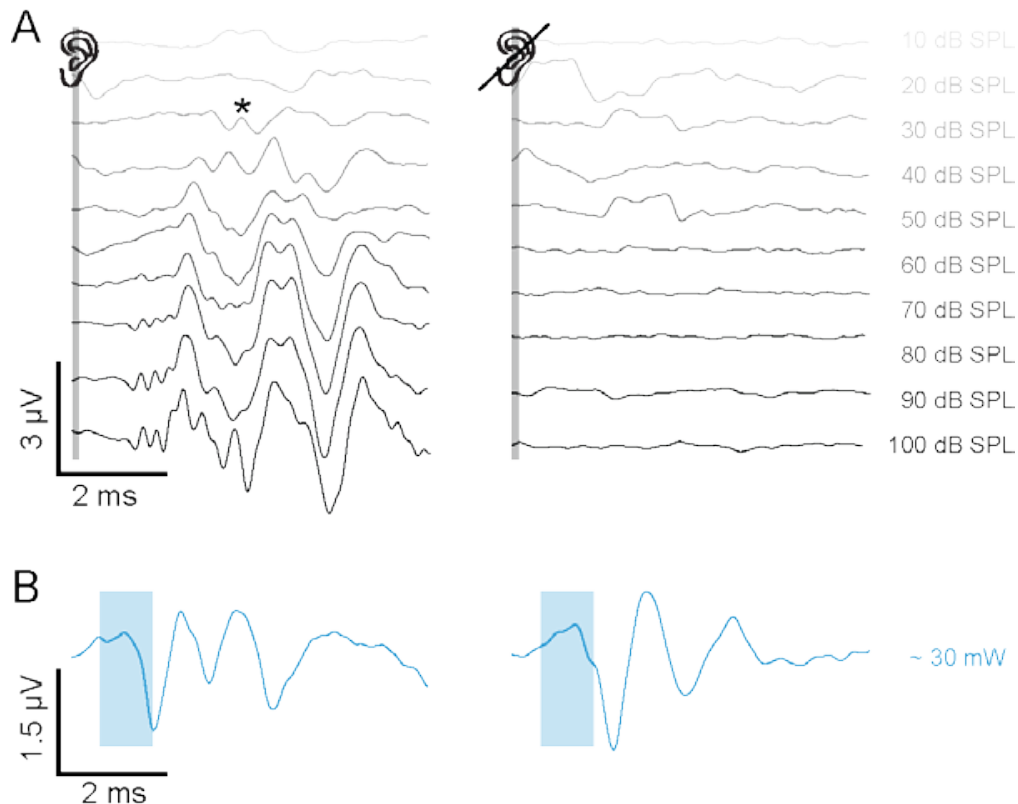

Supplementary Figure 2: Deafening by intracochlear kanamycin application (A) Auditory brainstem responses (average of 1000 stimulus presentations at a stimulation rate of $10 \mathrm{~Hz}$ ) upon click stimulation from 10-100 dB SPL before (left) and after deafening (right). The star indicates the threshold of acoustic stimulation. (B) Optically evoked auditory brainstem responses before (left) and after deafening (right), elicited by $\sim 35 \mathrm{~mW}$ pulses of $1 \mathrm{~ms}$ duration, delivered by a laser-coupled fiber (1000 stimuli presented at $10 \mathrm{~Hz}$ ). Optogenetic activation of the auditory system was still possible after deafening. Differences in amplitude and waveform before and after deafening are likely due to different positioning of recording electrodes and the optical fiber. 

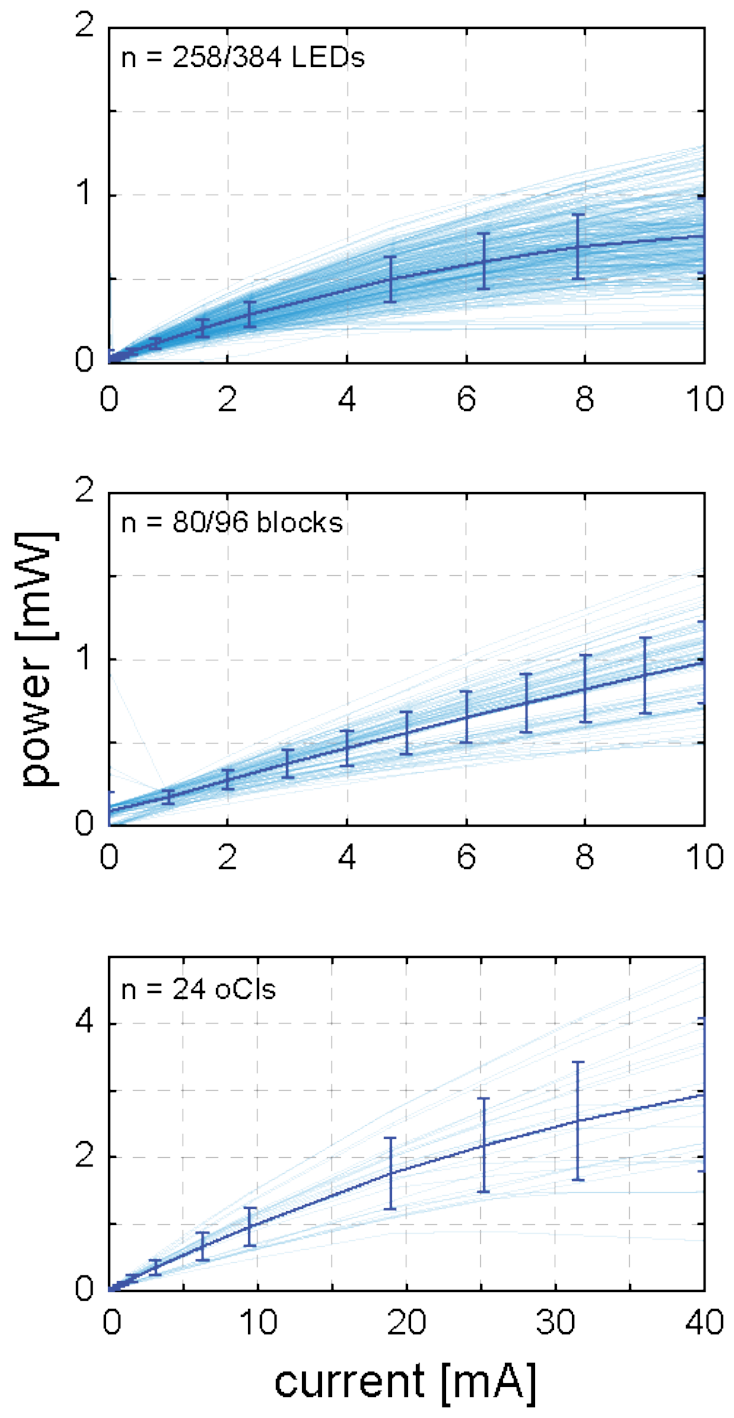

Supplementary Figure 3: Optical power of $\mu$ LED based oCls. The optical power of individual $\mu$ LEDs (top) and all $\mu L E D s$ on the oCl (bottom) has been measured before each experiment when driving the implant with up to $10 / 40 \mathrm{~mA}$, respectively. The power of four neighboring $\mu L E D s$ was estimated from the power of individual $\mu L E D s$ (up to $2.5 \mathrm{~mA} * 4$; center), since the driving current of $10 \mathrm{~mA}$ was distributed over all four $\mu \mathrm{LEDs}$. Non-functional $\mu L E D s$ (which did not emit light) have been excluded from analysis (numbers of functional/tested $\mu$ LEDs are indicated in each plot). 

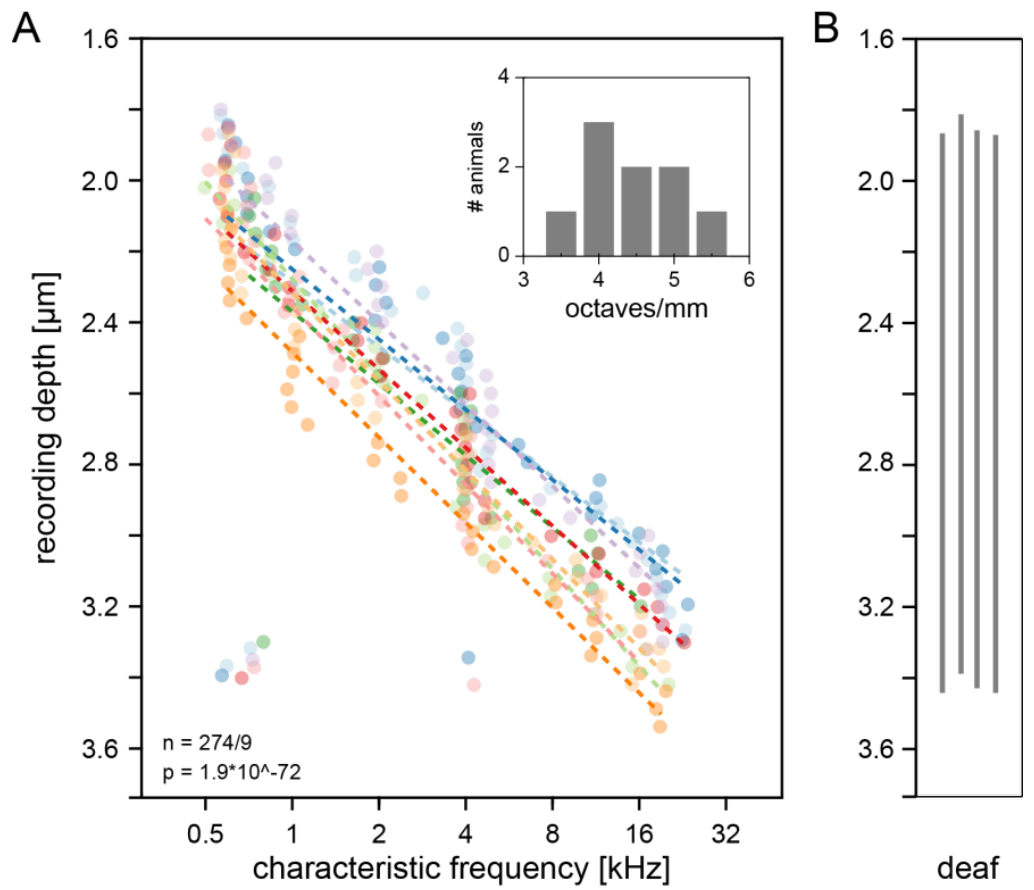

Supplementary Figure 4: Tonotopy in auditory midbrain. (A) Characteristic frequencies as a function of recording depth (normally distributed jitter of 0.05 octaves was added to each unit to reduce overlay of dta points for better visualization). Tonotopic slopes (dashed lines) were calculated by linearly fitting characteristic frequencies at given recording depths for each animal. Data from different animals is indicated by different colors. Inset: Distribution of tonotopic slopes of the 9 animals contributing to this study. (B) Electrode position in deafened animals. The silicon probe was placed as in normal hearing animals, but tonotopy of the ICC could not be mapped. 


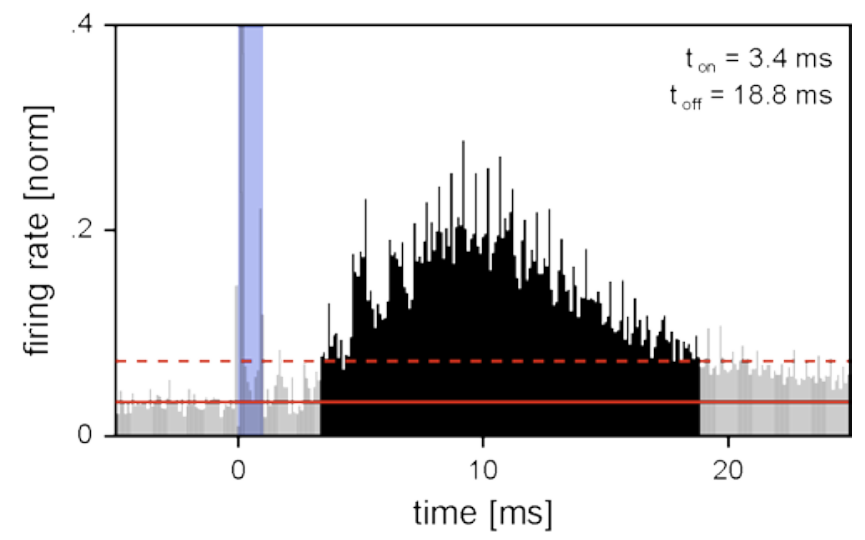

Supplementary Figure 5: Response window of oCl evoked responses. Peri-stimulus time histogram in response to SGN stimulation with 16 active $\mu$ LEDs of an oCl at maximum intensity ( $2 \mathrm{~mW}$ ), composed of multi-units recorded from all recording sites. Stimulus presentation is indicated in blue. Solid and dashed red lines indicate the mean firing rate plus 3 standard deviations, respectively. Bin size was set to $0.1 \mathrm{~ms}$, and the detected neural response is indicated in black. 

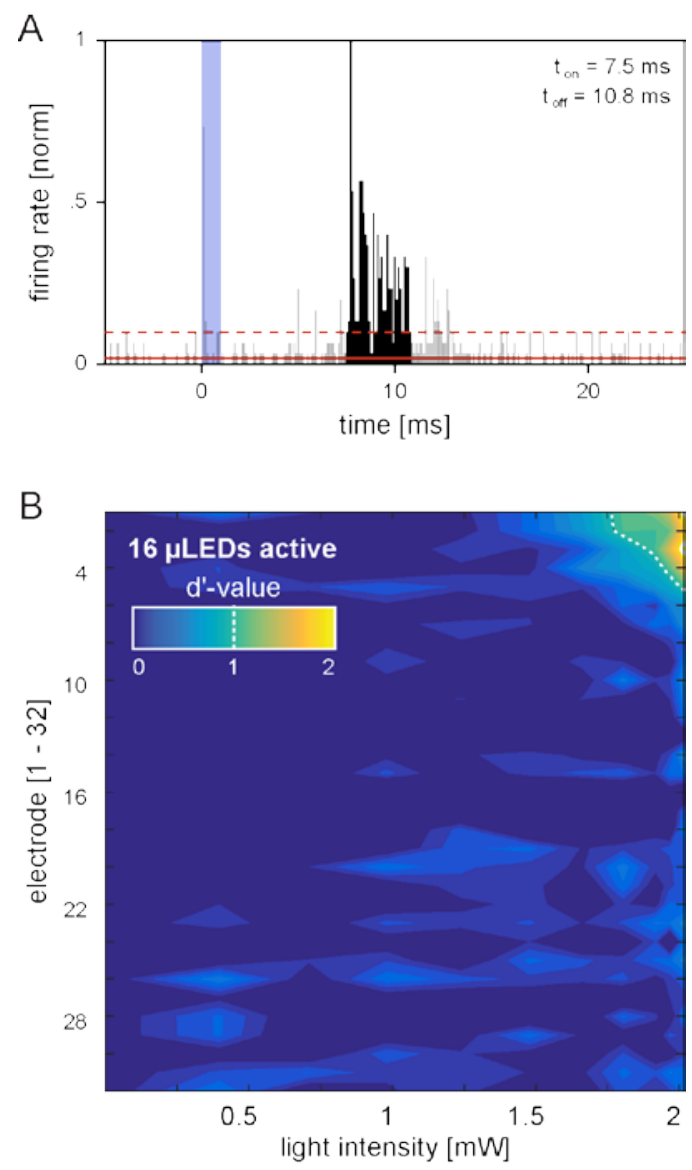

Supplementary Figure 6: $\mathrm{OCl}$ responses in noninjected gerbils. (A) PSTH in response to SGN stimulation with 16 active $\mu$ LEDs of an oCl at maximum intensity $(\sim 2.9 \mathrm{~mW})$, composed of multiunits recorded from responsive recording sites. Stimulus presentation is indicated in blue. Solid and dashed red lines indicate the mean firing rate plus 3 standard deviations, respectively. Bin size was set to $0.1 \mathrm{~ms}$, and the detected neural response is indicated in black. Responses have longer latencies when compared to $\mathrm{oCl}$ stimulation in virally transduced animals (compare to supplementary figure 2). (B) Exemplary STCs in response to SGN illumination with all $\mu$ LEDs in a non-injected gerbil. Responses are much weaker as compared to injected animals and do not correspond to the pattern of SGN illumination, as responses occur in the dorsal, low frequency regions of the ICC, even though basal, high frequency regions of the cochlea were stimulated (compare to figure $2 \mathrm{~A}, \mathrm{~B}$ ). 


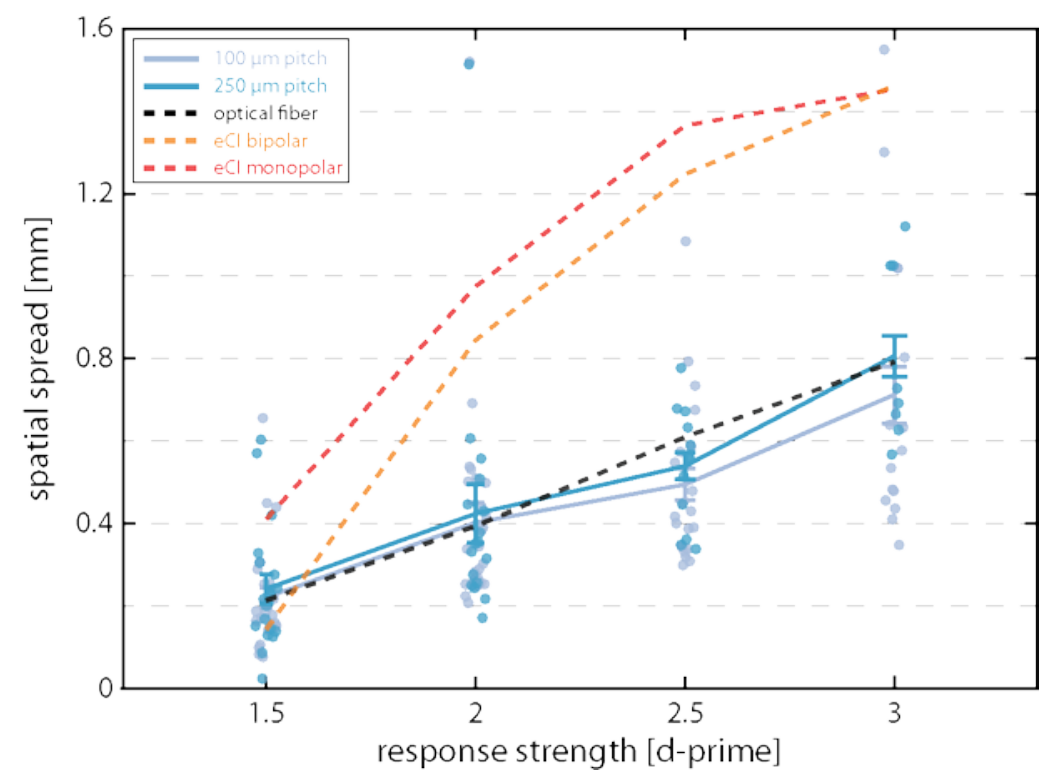

Supplementary Figure 7: Spatial spread of excitation. Spread of ICC activity upon SGN stimulation with $\mu$ LED-based oCIs (solid lines; mean \pm SEM), lasercoupled optical fibers, as well as mono- and bipolar electrical stimulation with a clinical-style eCI (dashed lines). Data of stimulation via optical fibers and electrical CIs is reprinted from ${ }^{20}$. 


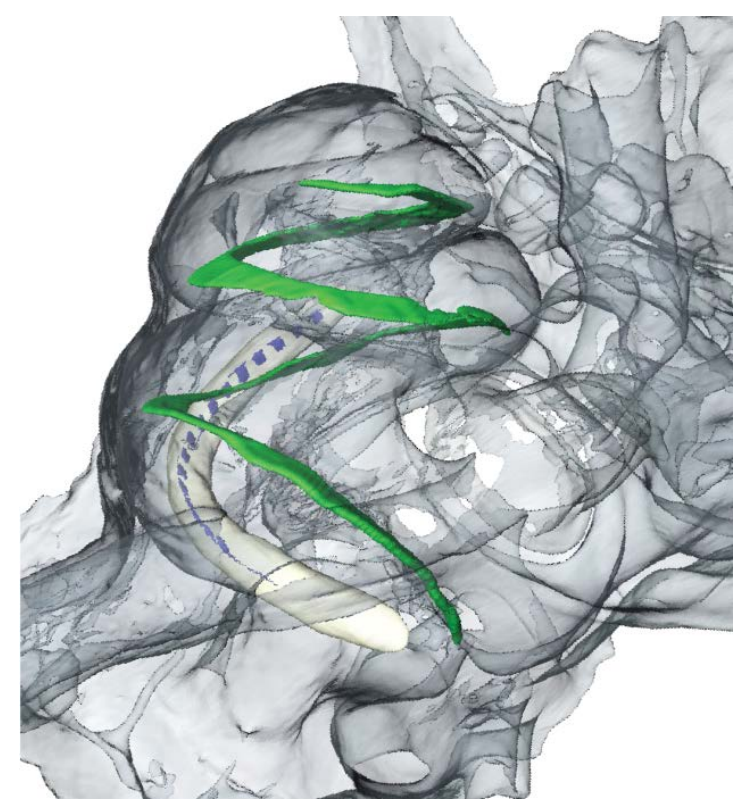

Supplementary Figure 8: Tomogram of the oCIimplanted cochlea. X-ray based $3 \mathrm{D}$ reconstruction of a gerbil cochlea implanted with a 16-channel $\mu$ LED-based oCI. Blue: $\mu$ LEDs; yellow: Polymer carrier substrate of oCI; green: basilar membrane. 


\section{Discussion}

\section{Cochlear optogenetics - current status}

The development of cochlear optogenetics as an alternative to electrical hearing restoration is a challenging, interdisciplinary task and multiple objectives must be met before considering clinical translation. A first requirement for optogenetic SGN stimulation is light sensitivity of the auditory nerve. Towards this end, potential molecular mediators for optogenetic stimulation have been identified and optimized for the auditory system using mice as a model system (Hernandez et al., 2014; Keppeler et al., 2018; Mager et al., 2018). Furthermore, temporal properties of optogenetic sound encoding have been characterized in these animals. Second, the technology to enable spatially confined illumination of the auditory nerve needs to be developed. First versions of multi-channel oCls have been engineered and their mechanical, thermal, and optical properties have been characterized (Goßler et al., 2014; Klein et al., 2018, 2019; Schwaerzle et al., 2016). Even though molecular as well as technological prerequisites are already advanced, both require further optimization. Moreover, the integration of both aspects and a thorough characterization of optical sound encoding in the auditory system, as well as behaviorally relevant perception remained important tasks to be performed. In this thesis, some of these aspects have been addressed:

First, a fiber-based single-channel oCl has been developed and implanted to the cochlea of adult Mongolian gerbils whose auditory nerve has been virally transduced to express the ChR-variant CatCh (Wrobel et al., 2018). By optogenetically stimulating the auditory nerve with this implant, the perception of optogenetic SGN stimulation has been demonstrated on the behavioral level (Wrobel et al., 2018). Furthermore, restoration of auditory function has been demonstrated on the physiological and behavioral level in a gerbil Model of sensorineural hearing loss (Wrobel et al., 2018). Both the perception of optogenetic SGN stimulation and the functionality of cochlear optogenetics in the absence of hair cells are important aspects when considering artificial sound encoding by optogenetics in humans suffering from sensorineural hearing loss.

Second, improved spectral selectivity of optogenetic over electric auditory nerve stimulation has been demonstrated (Dieter et al., 2019). An activity-based analysis of multi-unit recordings in the auditory midbrain enabled comparison of the spectral selectivity upon acoustic, optogenetic and electric stimulation. It was shown that optogenetic SGN stimulation outperforms monopolar 
electrical stimulation at all activation levels, while it was spectrally more specific than bipolar electrical stimulation for SGN stimulation beyond threshold values (Dieter et al., 2019). At low stimulus intensities, the spread of excitation upon optogenetic SGN stimulation was even as narrow as the spread of excitation upon pure tone acoustic stimulation in wildtype animals (Dieter et al., 2019). This improvement of spectral selectivity is of uttermost importance, since the challenging development of oCls and their implementation in a clinical setting is only justified if a fundamental improvement of artificial sound encoding is to be expected.

Third, functional activation of the auditory system by 16 -channel $\mu$ LED-based optical cochlear implants has been demonstrated in Mongolian gerbils. Multi-channel recordings in the auditory midbrain of anesthetized animals revealed optogenetic SGN stimulation by individual $\mu$ LEDs, and the strength of midbrain activation positively correlated with the recruitment of additional $\mu$ LEDs in the cochlea. The spectral selectivity of $\mu L E D$-mediated optogenetic SGN stimulation outperformed electrical stimulation. These results demonstrate that $\mu$ LED-based oCls can achieve increased spectral selectivity as compared to eCls and are an important milestone towards the development of cochlear optogenetics for hearing restoration.

Even though these results promise improved hearing restoration by cochlear optogenetics in the future, a long way remains to be gone before considering clinical application. The following sections will briefly summarize the experimental evidence for improved sound encoding by cochlear optogenetics, before discussing remaining tasks towards clinical translation. Finally, optogenetic activation of the auditory system will be discussed in a broader context of auditory neuroscience. 


\section{Sound encoding by cochlear optogenetics}

Most real-world acoustic signals are complex, and the auditory system relies on multiple mechanisms to encode these signals. Spectral information is primarily encoded via tonotopic SGN activation, but a spike time code of neural activity contributes to coding of low frequencies. Sound intensity is encoded both on the single neuron and on the population level: When stimulus intensities are increased, this raises the firing rate of individual neurons, while additional neurons are recruited to code intensity on the population level. Finally, the temporal structure of sound is encoded by precise timing of action potentials. Thus, in order to enable restoration of most natural hearing, cochlear optogenetics needs to be spectrally selective (to encode sound frequency), enable a broad modulation of response strengths (to encode sound intensity), and be temporally precise (to not miss out on temporal and spectral properties of the acoustic stimulus).

Frequency resolution: Spectral selectivity of cochlear optogenetics was first shown by local field potential recordings and current source density analysis in the inferior colliculus of transgenic mice expressing ChR2 (Hernandez et al., 2014). While illuminating the cochlear base - which is coding for high frequencies - via the round window, neural activity was observed in high frequency regions of the auditory midbrain. The spread of neural activation was statistically indistinguishable from acoustic stimulation ( $31 \mathrm{kHz}$ pure tone, $80 \mathrm{~dB} \mathrm{SPL}$ ), and 1.74-fold more restricted than monopolar electric stimulation (Hernandez et al., 2014). In this thesis, spectral selectivity of cochlear optogenetics has been analyzed by multi-unit recordings from the ICC in Mongolian gerbils while stimulating the cochlea via three optical fibers at low, mid and high frequency regions. Upon illumination at a given site in the cochlea, activity has been observed in tonotopically corresponding ICC regions (Dieter et al., 2019). The breadth of neural activation in the ICC, which allows for the inference of the spread of excitation in the cochlea, was 2.04- and 1.94-fold as narrow as upon mono- and bipolar stimulation with a 4-channel clinical cochlear implant. The spread of excitation upon cochlear illumination with four neighboring optical emitters (100 $\mu \mathrm{m}$ pitch) of a 16-channel, $\mu$ LED-based optical $\mathrm{Cl}$ was estimated to be 2.37 and 2.26 fold as narrow as the spread of excitation upon mono- and bipolar electrical stimulation (values for electrical stimulation are taken of a comparable study using the same methods (Dieter et al., 2019)). While these estimates of spectral selectivity are highly promising and prove that optical sound encoding can indeed overcome the limitation of electrical cochlear implants, these results should be confirmed also in other model organisms, ideally in the awake state. Furthermore, future experiments should involve optical cochlear implants with larger numbers of independent 
emitters. A higher density of emitters along the cochlear axis will be important to determine the frequency resolution limit of optical cochlear implants, and might demonstrate the advantage over eCls even stronger, since a similar implant design enables better comparison of the two stimulation modes. If the resolution limit of oCls would overcome the ones of eCls also in these settings, and the number of independent stimulation sites can be substantially increased, the objective of clinical translation of cochlear optogenetics would seem justified.

Modeling studies (based on X-ray imaged gerbil cochleae, literature values of optical parameters of biological tissues, and optical fiber properties identical to the experimental setup reported in this thesis) corroborated the high spectral selectivity of optical sound encoding: Monte Carlo ray tracing confirmed the feasibility of spatially precise auditory nerve stimulation, given appropriate positioning of the light emitters and adequate illumination intensities (Dieter et al., 2019; Wrobel et al., 2018). Modelling furthermore suggested that spectral selectivity might be improved even further by optimizing various aspects of SGN illumination: On the engineering level, the surface of the light emitter might be further decreased, which consequently would lead to a decrease in the illuminated area at the level of the spiral ganglion. In addition, lower numerical apertures of the light emitters could improve the confinement of the beam path. Since the geometry of the light emitting surface relative to the neural target structure is of critical importance for spatially selective neural activation, improvements might also be achieved on the experimental side: First, the distance of the stimulators to the target tissue should be decreased, which can be done by intracochlear emitters wrapping around the modiolus. Second, the emitter surface should be directed as orthogonal as possible to the spiral ganglion. Illumination should target the somata of SGNs rather than the peripheral neurites, since the preservation of the latter upon hearing loss might be reduced. By optimizing the emitter placement in these ways, the illuminated area at the level of the spiral ganglion can be minimized (Dieter et al., 2019; Wrobel et al., 2018). Finally, the implementation of opsins with a red-shifted action spectrum might increase spectral resolution of optical sound encoding, since light scattering by biological tissues is less pronounced for red light as compared to blue light and thus the confinement of the light beam might be more narrow (Jacques, 2013).

Together these results demonstrate increased spectral selectivity of optogenetic over electric SGN activation and thus suggest that oCls can indeed overcome the major limitations of eCls and hence increase frequency resolution of hearing restoration. 
Dynamic range: Besides frequency information, also intensity information is limited in electrical sound encoding. Since SGNs have an intrinsically low dynamic range in response to electric current (1-2 dB (Miller et al., 2006)), and large SGN populations are activated at similar thresholds, the output dynamic range of electrical cochlear implants is limited to typically 10-20 dB (Miller et al., 2006; Rubinstein, 2004), as compared to 120 dB that can be coded by acoustic stimuli (Viemeister, 1988). Based on the stimulus-response function of oABR wave I amplitudes, several studies on cochlear optogenetics have reported dynamic ranges of 10-20 dB (Keppeler et al., 2018; Mager et al., 2018; Wrobel et al., 2018). On the level of the auditory midbrain, the dynamic range of optogenetic SGN stimulation has been estimated to be 7-8dB (and $\sim 11 \mathrm{~dB}$ on the population level, which was comparable to the dynamic range of mono- and bipolar electrical SGN stimulation), based on the activity of multi-neuronal clusters (Dieter et al., 2019). These values slightly exceeded the dynamic range reported from eCl stimulation in the rat (also based on multi-unit activity in the inferior colliculus) which have been reported to be between 6-8 $\mathrm{dB}$ (Allitt et al., 2012, 2016). Two notions should be taken when interpreting these results: First, it is likely that the true output dynamic range of optogenetic SGN stimulation has been underestimated. This is evident from the fact that most neural responses did not reach saturation, even at the highest stimulation intensities tested (Dieter et al., 2019). Second, adequate comparison between optogenetic and electrical stimulation is not straightforward: The dynamic range of electrical SGN activation was calculated based on current amplitude, while the dynamic range of optogenetic SGN activation was calculated based on optical power. Calculating the dynamic range based on power versus amplitude leads to a difference by the factor of two. The power-based calculation is likely to be appropriate for optogenetic stimulation, since the optical power directly relates stimulation intensity to membrane depolarization of SGNs. However, the unit that relates stimulus intensity to SGN depolarization when stimulating electrically is likely to be electric charge rather than current amplitude. With this assumption, the dynamic range of electrical stimulation has been overestimated by a factor of two, and hence the dynamic range of optogenetic stimulation might outperform electrical stimulation. The maximum output dynamic range that can be coded optically will further be influenced by additional factors: The implementation of future opsins with increased light sensitivity, as well as optimized emitter positioning, might lower the light thresholds for SGN activation and hence increase the dynamic range at the lower end of coding. However, long-term safety limits for tissue illumination to avoid heating and phototoxicity might limit the maximum stimulus intensity, and hence the dynamic range at the upper end of coding. To finally answer the question regarding the output dynamic 
range of cochlear optogenetics, and furthermore to address the question how many intensity steps might be discriminable, also behavioral experiments are needed.

Temporal coding: Coding of acoustic stimuli in the auditory system heavily relies on spike timing, since SGN typically have firing rates up to several hundreds of Hertz with sub-millisecond precision (up to $\sim 100 \mathrm{~Hz}$ spontaneous activity and up to $\sim 400 \mathrm{~Hz}$ sustained rates of acoustically evoked responses) (Heil and Peterson, 2015; Liberman, 1978; Schmiedt, 1989). Therefore, optogenetic SGN stimulation must facilitate action potential generation in SGNs with great temporal fidelity even at high stimulation rates. Temporal fidelity of optogenetic neural activation depends on the kinetics of the expressed opsin, and the high frequency cut-off for light-induced spiking is mainly governed by the time constant $\tau_{\text {off }}$ for channel closing after illumination is terminated. Since optogenetic tools with improved kinetics are of broad interest in the neuroscience community, quite some effort was put on the identification of faster opsin, both by screening of naturally occurring opsins (Klapoetke et al., 2014) and by directed mutagenesis of previously described opsins (Gunaydin et al., 2010; Mager et al., 2018). The temporal fidelity of optogenetic SGN stimulation has been approximated on the population level by oABR recordings and on the single unit level by auditory nerve fiber recordings for the ChRs ChR2 ( $\tau_{\text {off }}=10 \mathrm{~ms}$ at room temperature (Hernandez et al., 2014)), CatCh ( $\tau_{\text {off }}=16 \mathrm{~ms}$ (Kleinlogel et al., 2011; Wrobel et al., 2018)), Chronos ( $\tau_{\text {off }}=3.6 \mathrm{~ms}$ (Keppeler et al., 2018; Klapoetke et al., 2014)), and f-Chrimson $\left(\tau_{\text {off }}=5.7 \mathrm{~ms}\right.$ (Mager et al., 2018)). However, with increasing temperature, closing kinetics of ChRs are scaled by a temperature coefficient $\left(\mathrm{Q} 10=\right.$ scaling factor for a temperature change of $\left.10^{\circ} \mathrm{C}\right)$. In the case of Chronos, the Q10 amounts to 2.7 ( $\tau_{\text {off }}=0.76 \mathrm{~ms}$ at body temperature), and a Q10 of 2.4 has been reported for ChR2 ( $\tau_{\text {off }}=3 \mathrm{~ms}$ at body temperature (Keppeler et al., 2018)). Assuming similar Q10 values (e.g. 2.5) for CatCh and f-Chrimson, their closing kinetics would be as fast as 4.6 $\mathrm{ms}$ and $1.6 \mathrm{~ms}$ at physiological temperature.

Since ABRs are compound potentials whose amplitude is determined both by the amount of activated neurons and by their synchronization, the amplitude and temporal jitter of oABR wave I, which originates from activity in the auditory nerve, serves as a proxy for temporal fidelity of optogenetic SGN activation on the population level (Henry, 1979; Land et al., 2016). Typically, the amplitude of $O A B R$ wave I decreases and latency as well as its jitter rise with increasing stimulation rates, indicating the limitation of temporal fidelity of coding (Hernandez et al., 2014; Keppeler et al., 2018; Mager et al., 2018; Wrobel et al., 2018). In ChR2-mediated responses, 
oABRs could not be evoked at stimulation rates greater than $80 \mathrm{~Hz}$ (Hernandez et al., 2014), while stimulation rates of up to $200 \mathrm{~Hz}$ still were able to evoke responses in CatCh- and f-Chrimson expressing animals (Mager et al., 2018; Wrobel et al., 2018). Using Chronos, responses could be evoked even with stimulation rates up to $1 \mathrm{kHz}$, although the signal propagation along the pathway could not be validated given the brief averaging time window (Keppeler et al., 2018).

On the level of individual SGNs, temporal fidelity of cochlear optogenetics was estimated by the spike probability and the vector strength - a measure for the quality of phase-locking - of lightinduced action potentials. In CatCh-transduced gerbils, both spike probability and vector strength decreased substantially at stimulation rates beyond $100 \mathrm{~Hz}$ (Wrobel et al., 2018), while fChrimson- and Chronos-transduced mice facilitated spike probabilities of $\sim 0.3$ even at stimulation rates up to $200 \mathrm{~Hz}$ and substantial vector strength up to rates of a few hundred Hertz (and even up to $1 \mathrm{kHz}$ in some Chronos-expressing SGNs) (Keppeler et al., 2018; Mager et al., 2018).

Even though the implementation of opsins with improved kinetics enables reliable and precise spike generation up to few hundreds of Hertz, the temporal fidelity of natural sound encoding is not yet reached. However, it has been shown that speech recognition mainly relies on temporal cues in the range of $50-160 \mathrm{~Hz}$, which would be covered by both Chronos and f-Chrimson (Shannon et al., 1995). Furthermore, some portion of the temporal limitation might be compensated by the auditory nerve on the population level, since several SGNs jointly encode information (Liberman, 1978). Nevertheless, the search for faster opsins should further continue in order to identify even more suitable opsins for future applications in hearing restoration. 


\section{Cochlear optogenetics for hearing restoration}

Besides encoding acoustic stimuli on the physiological level, cochlear optogenetics must meet some further requirements to qualify as an alternative method for the treatment of hearing loss: First, opsin expression must be stable over time, in order to enable SGN activation by light over decades, and ideally over the whole lifespan of the patient. Second, in order to allow for cognition of optically coded auditory information, optogenetic SGN stimulation must not only drive the auditory system physiologically, but must also evoke a percept. Third, optogenetic SGN stimulation must be functional in the absence of hair cells, i.e. in the deafened cochlea, since most patients suffer from hearing loss due to dysfunction of inner hair cells. Finally, optical sound encoding should happen at reasonable thresholds, minimizing the energy requirements and thus increasing battery lifetime of future oCls.

\section{Long term stability of opsin expression:}

By chronic implantation of optical fibers into the cochlea and weekly recordings of oABRs, this thesis demonstrates that cochlear optogenetics can elicit stable physiological responses over more than 110 days, which was the longest period observed in these experiments (Wrobel et al., 2018). Long term stability of acutely recorded oABRs has also been shown in different studies in mice: One study demonstrated that optogenetic stimulation of SGNs which express f-Chrimson evoked comparable oABRs in young mice and in mice were expressing the opsin for nine months (Mager et al., 2018). A second study reported similar opsin expression levels and comparable oABRs 6-18 weeks after transduction of the auditory nerve of mice with Chronos (Duarte et al., 2018). However, studies that analyze functional opsin expression over years, or ideally over the whole lifespan of an animal, are still pending.

\section{Perception of cochlear optogenetics:}

An optogenetically evoked percept strong enough to guide avoidance behavior in Mongolian gerbils has been demonstrated in this thesis (Wrobel et al., 2018). By chronically implanting optical fibers into the cochlea and training implanted animals in the shuttle-box paradigm of negative reinforced learning, animals could demonstrate the perception of optogenetic SGN stimulation via locomotion. Minimal energy thresholds required to evoke a percept were as low as $1.3 \mu \mathrm{J}$, which still exceeds the energy requirements for electrical cochlear implants ( $0.2 \mu \mathrm{J}$ for a pulse of $80 \mu \mathrm{s})(Z i e r h o f e r$ et al., 1995). After learning the behavioral task with optogenetic cues, gerbils could generalize and transfer that knowledge to acoustic cues, suggesting some degree of similarity between perception of optogenetic and acoustic stimuli (Wrobel et al., 2018). A 
different study confirmed the behaviorally relevant perception of optogenetic stimulation at a different stage in the auditory system: Upon virus injection (with constructs coding for ChR2 or Chronos) and fiber implantation in the inferior colliculus of mice, animals were successfully trained in the shuttlebox and could indicate the perception of optogenetic stimulation of the auditory midbrain (Guo et al., 2015).

\section{Optogenetics in the deafened cochlea:}

This thesis demonstrates optogenetic activation of the auditory nerve independent of hair cell function (Wrobel et al., 2018). As a model of sensorineural hearing loss, gerbils have been pharmacologically deafened by local application of aminoglycosides to the inner ear, and deafness has been proven both behaviorally and by ABR recordings. Even though hair cells were lost after deafening, as demonstrated by post-mortem histological analysis, optogenetic SGN stimulation was still able to evoke oABRs, and animals were successfully trained to solve the task by using optogenetic cues. Re-activation of the deafened cochlea by optogenetics was already demonstrated before for different models of deafness: As proven by recordings of oABRs, cochlear optogenetics could activate the auditory nerve in a ChR2-expressing mouse model of human deafness (Hernandez et al., 2014), in which transmitter release from inner hair cells is severely impaired (Pangrsic et al., 2010). Furthermore, optogenetic auditory nerve activation was demonstrated after the injection of furosemide (Hernandez et al., 2014), which prohibits mechanotransduction by hair cells due to the abolishment of the endocochlear potential (Sewell, 1984). A different study reported optogenetic SGN stimulation (Mager et al., 2018) in a mouse model of age related hearing loss (Shnerson et al., 1981): OABRs recorded from nine months old C57BL/6J mice, whose aABR amplitudes only amount to one third of the aABR amplitude found in young mice, and whose aABR thresholds are elevated by almost $40 \mathrm{~dB} S P L$, were still comparable to the oABRs recorded in young mice (Mager et al., 2018). Taken together, these studies convincingly demonstrate the feasibility of optogenetic SGN stimulation in various rodent models of deafness, including genetic, drug-induced, and age-related loss of inner hair cells.

\section{Thresholds of neural activation:}

A further consideration for hearing restoration by optogenetics is the energy required for optical sound encoding. Activation thresholds must be minimized to enable safe stimulation and avoid phototoxicity or heating in the cochlea, but also to achieve reasonable battery lifetimes when using future oCls in daily life. Thresholds of optogenetic SGN activation generally depend on multiple aspects, such as the expression level of the opsins, their open channel lifetime, and 
emitter placement, while the light sensitivity of the chosen channelrhodopsins seems relatively constant. In this thesis, SGNs have been optogenetically modified to express CatCh, and thresholds for neural activation have been determined with multiple methods: multi-unit recordings in the ICC revealed thresholds of $2.67 \mathrm{~mW}$ for $1 \mathrm{~ms}$ pulses, which corresponds to an energy of $2.67 \mu$ J (Dieter et al., 2019), while activation thresholds of single units in the auditory cortex (recorded in high frequency regions, and thus matching the fiber placement in the cochlea cochlea) were as low as $1.4 \mu \mathrm{J}$ (1.4 mW at $1 \mathrm{~ms}$ pulse duration) (Wrobel et al., 2018). Population potentials (oABRs) were reported to have higher activation thresholds (on average $4.6 \mu \mathrm{J}$ ), while behavioral thresholds were as low as $1.8 \mu \mathrm{J}$ (Wrobel et al., 2018). Studies on SGNs expressing different opsins have reported oABR thresholds of 6-9 $\mu$ J (Chronos (Duarte et al., 2018; Keppeler et al., 2018)), $4.6 \mu \mathrm{J}$ (Chronos-ES/TS (Keppeler et al., 2018)), $2.2 \mu \mathrm{J}$ (ChR2 (Hernandez et al., 2014)), and even as low as 0.5-1 $\mu \mathrm{J}$ when transducing the auditory nerve with f-Chrimson (Mager et al., 2018). From these estimates, f-Chrimson is a promising candidate for optogenetic hearing restoration, since it enables safe SGN activation not only due to low thresholds, but also due to its red-shifted action spectrum (Mager et al., 2018). However, even the energy required to optically excite auditory neurons expressing f-Chrimson still exceeds the energy requirements for electrical SGN stimulation ( $0.2 \mu \mathrm{J}$ for a pulse of $80 \mu \mathrm{s}$ ) (Zierhofer et al., 1995). Fortunately, the optogenetic toolbox is under active investigation, and tools with optimized characteristics are frequently discovered, raising the hope that opsins with increased light sensitivity might be available soon. As stated earlier, also more ideal placement of the light sources (Dieter et al., 2019; Wrobel et al., 2018) and higher levels of opsin expressions (Keppeler et al., 2018) might contribute to further lower the energy requirements for optogenetic sound encoding. 


\section{Engineering of multi-channel optical cochlear implants}

While considerable progress has been made on the biological side of cochlear optogenetics in recent years, also the engineering of multi-channel optical stimulators for cochlear illumination reported great advancements. The design and technical realization of oCls is very demanding, and heavily restricted by the cochlear anatomy: Implants must be scaled to fit into the limited intracochlear space, and flexible enough to follow the cochlear turns without causing trauma due to implantation. Large numbers of optical emitters should be integrated on this carrier, and the implant must be embedded in a biocompatible encapsulation that guarantees long-term stability over decades, while being both transparent and electrically insulating (in the case of $\mu$ LED-based oCls). Furthermore, the design and optimization of the emitter itself is very challenging: light sources should have a narrow beam profile to allow for confinement of the light, while providing sufficient power output at minimal energy consumption. Two general designs can be considered (Dombrowski et al., 2018; Jeschke and Moser, 2015): passive implants, which consist of intracochlear waveguides coupled to external light sources (Balster et al., 2014), and active implants, whose optoelectronic components are integrated on the carrier and placed in the cochlea close to the neural target tissue (Goßler et al., 2014; Klein et al., 2018; Schwaerzle et al., 2016; Xu et al., 2018). Passive implants have the advantage to spatially separate the light source from the cochlea. Optoelectronic components could be placed in a hermetically sealed housing, which would have several advantages. Spatial separation of optoelectronics and tissue would decrease the risk of tissue damage due to heating, which is a big advantage in terms of patient safety. Furthermore, the encapsulation of the intracochlear part would be less critical, the size of light sources is less limited, and light sources could potentially be exchanged in case an emitter would fail. On the other hand, oCls based on active emitters would be favorable in terms of energy consumption, since passive stimulators face loss of light at the interface between the light source and the waveguide, as well as along the waveguide (Alt et al., 2017).

\section{Passive optical cochlear implants:}

So far, only one passive oCl has been developed, which is composed of eight silicone-embedded glass fibers of $25 \mu \mathrm{m}$ diameter (Balster et al., 2014). This implant has successfully been inserted into explanted human temporal bones, without causing any trauma due to implantation. Furthermore, insertion forces for this $\mathrm{OCl}$ have been assessed in a 2D cochlea model and were comparable to the forces observed when inserting conventional electrical Cls (Balster et al., 2014). While this device is already very promising, it is not yet functional, since it was not coupled 
to optical emitters, and the number of waveguides must be substantially increased. The latter might be achieved by the implementation of single-mode optical fibers, which typically are thinner (2-10 $\mu \mathrm{m}$ diameter (Alt et al., 2017)) than the multi-mode fibers used in this study (typically tens to hundreds of $\mu \mathrm{m}$ (Balster et al., 2014)). A second advantage of single mode fibers is the lower numerical aperture, which narrows the beam of out coupled light and would thus enable neural stimulation with greater spatial selectivity (Alt et al., 2017). Unfortunately, singlemode fibers come at the cost of more challenging light coupling the emitter-waveguideinterface (Alt et al., 2017). Alternatively, fibers made from polymers might serve as waveguides for passive optical Cls, since they can be manufactured with diameters below $10 \mu \mathrm{m}$ (Alt et al., 2017; Xu et al., 2018; Zorzos et al., 2010). Furthermore, polymer-based waveguides, even though being less light efficient than glass fibers, might be beneficial in terms of their mechanical properties, since they are more flexible than glass fibers, which could be of advantage when winding along the cochlear spiral.

\section{Active optical cochlear implants:}

Active oCls based on LEDs have been developed in various versions during the past few years (Goßler et al., 2014; Klein et al., 2018, 2019; Schwaerzle et al., 2016; Xu et al., 2018). In one approach, ten commercially available LEDs $(220 \times 270 \mu \mathrm{m} ; 460 \mathrm{~nm}$ emission peak; Cree Europe $\mathrm{GmbH}$ ) have been flip-chip bonded on a flexible polyimide substrate with a pitch of either 350 or $500 \mu \mathrm{m}$ (Schwaerzle et al., 2016). The oCl of $20 \mathrm{~mm}$ length and $0.24 \mathrm{~mm}$ width allowed for a bending radius of $1 \mathrm{~mm}$, while still being functional. Individually addressable LEDs had an output power of $0.3 \mathrm{~mW}$ (when driven at $5 \mathrm{~mA}$ ), which amounts to a power density of $\sim 5 \mathrm{~mW} / \mathrm{mm}^{2}$ (Schwaerzle et al., 2016), which is sufficient to drive most opsins (Deisseroth and Hegemann, 2017). When placed on agarose and driven at a duty cycle of $10 \%$, the implant led to a maximum temperature increase of $1.67^{\circ} \mathrm{C}$. In a different approach, 15 LEDs $(1 \times 0.6 \mathrm{~mm}, 470 \mathrm{~nm}$ emission peak) with a maximum output of $34 \mathrm{~mW}$ have been embedded in biocompatible silicone and inserted into models of the human cochlea (Xu et al., 2018). Insertion forces have been assessed and were comparable to forces observed upon implantation of electrical cochlear implants. Besides cochlear application, wireless controlled LEDs have been engineered for optogenetic experiments with emission peaks in the red, yellow, green, blue $(220 \times 270 \mu \mathrm{m}$ each $)$ and ultraviolet $(100 \times 100 \mu \mathrm{m})$ spectrum (Shin et al., 2017). Even though these designs are important steps towards the development of optical cochlear implants, commercially available LEDs are still relatively large, and thus the number of emitters on the implant is limited. 
To further miniaturize optical emitters, Gallium Nitride ( $\mathrm{GaN}$ ) based thin film $\mu$ LEDs as small as 50 x $50 \mu \mathrm{m}$ have been developed and integrated on flexible substrates (Goßler et al., 2014; Klein et al., 2018). GaN depicts the ideal material for $\mu$ LED fabrication since it is biocompatible (Jewett et al., 2012) and highly stable (lifetimes of up to 100,000 hours have been reported)(Alt et al., 2017; Laubsch et al., 2010). Furthermore, the emission spectrum of GaN-based $\mu$ LEDs ranges from green to ultraviolet light, and power efficiencies as high as $60 \%$ can be achieved (Alt et al., 2017; Laubsch et al., 2010). First developments of $\mu L E D$-based oCls for animal experiments resulted in two different designs (Goßler et al., 2014): One oCl, with dimensions of $0.23 \times 5 \mathrm{~mm}$, carried four $\mu L E D s$ of $50 \times 50 \mu \mathrm{m}$. A second oCl of $0.38 \times 5 \mathrm{~mm}$ size carried $15 \mu$ LEDs of $150 \times 150 \mu \mathrm{m}$. $\mu$ LEDs had an emission peak of $405 \mathrm{~nm}$ and a maximum output of $60 \mu \mathrm{W}$ when driven with a current of 1 $\mathrm{mA}$, corresponding to a power density of $6 \mathrm{~mW} / \mathrm{mm}^{2}$. While the second implant was successfully inserted into the model of a rat cochlea, the smaller design of the first implant even allowed implantation into an explanted mouse cochlea via the round window (Goßler et al., 2014). Further advancement of these oCls led to an implant which houses 144 individually addressable $\mu$ LEDs of $50 \times 50 \mu \mathrm{m}$ (emission peak: $462 \mathrm{~nm}$; $\max$. power $0.82 \mathrm{~mW},=4.07 \mathrm{~mW} / \mathrm{mm}^{2}$ ) at a total size of 0.35 x $15 \mathrm{~mm}$ (Klein et al., 2018). A fully epoxy-based, biocompatible carrier substrate of these implants minimized thermomechanical bending, and maximum temperature increases of $1{ }^{\circ} \mathrm{C}$ when driving the implant at $10 \mathrm{~mA}$ for $20 \mathrm{~ms}$, enabling safe in vivo application of this implant (Klein et al., 2018). Indeed, functional activation of the auditory nerve by oCls of this kind has been successfully demonstrated in this thesis. Follow-up efforts to improve the optical properties of oCls employed conical concentrators and spherical micro-lenses made from polydimethylsiloxane (a transparent, flexible and biocompatible material), which were placed on the light emitting surface of $\mu$ LEDs (Klein et al., 2019). Concentrators and micro-lenses increased light extraction by $83 \%$ and peak intensity by $95 \%$, promising more robust opsin activation, but also lowering energy consumption and temperature increase during illumination, as compared to identical SGN illumination without micro-lenses (Klein et al., 2019). 


\section{The roadmap towards clinical translation of optogenetic cochlear implants}

Despite the enormous progress both in animal experiments and in oCl engineering, lots of work remains to be done before considering clinical translation of cochlear optogenetics. This section will outline the major objectives that should be addressed in future studies in order to pave the way for clinical translation.

\section{Rendering the auditory nerve light sensitive}

\section{Choosing the ideal opsin for cochlear optogenetics:}

To facilitate optogenetics-based artificial sound encoding with high temporal fidelity, at low thresholds, and with minimal risk for phototoxicity, the ideal opsin would combine a red-shifted action spectrum, fast opsin kinetics, large ion conductance, and high light sensitivity. The redshifted action spectrum is desired because red light is reportedly less phototoxic than blue light (Kerstein et al., 2014; Mager et al., 2018). Furthermore, due to reduced absorption, red light penetrates deeper into biological tissue, lowering the light thresholds needed for opsin activation (Jacques, 2013). Both the reduction in phototoxicity and the lower illumination intensities would increase the biosafety of cochlear optogenetics. Finally, also the scattering in biological tissue is less pronounced for red than for blue light, which potentially enables improved spatial confinement of the beam path, enhancing spatial selectivity of neural activation and thus increasing frequency resolution of oCls (Jacques, 2013). As discussed previously, temporal fidelity of optogenetics-mediated action potential generation is mainly limited by the closing constant $\tau_{\text {off }}$ of a channel. Even though the fastest opsins known to date are approaching physiological firing rates of SGNs, some improvement is still needed. This might be achieved by directed mutagenesis: helix 6 mutations in the red-shifted opsin Chrimson considerably sped up $\tau_{\text {off }}$ and resulted in the versions $\mathrm{f}$-Chrimson and vf-Chrimson ( $\tau_{\text {off }}=3.2 \mathrm{~ms}$ and $1.6 \mathrm{~ms}$ at body temperature, respectively, as compared to $\sim 10 \mathrm{~ms}$ in regular Chrimson) (Mager et al., 2018). These mutations could in principle also be done in other opsins, such as the fastest natural occurring opsin, Chronos, to speed up their channel kinetics (Klapoetke et al., 2014). Unfortunately, shorter lifetimes of opened channels due to faster closing reduce photocurrents in the target cell, which leads to decreased light sensitivity of the cell. Consequently, light sensitivity and channel kinetics must be balanced. Increasing the ion conductance of the opsin should be considered in order to mediate strong and stable depolarization of transduced cells and thus 
facilitate reliable responses to neural illumination.. Thus, the amount of foreign protein necessary to mediate responses could be reduced, consequently reducing stress on the target cell. Finally, it might be worth to consider light-activated ion pumps, such as Xenorhodopsin, in order to decouple the ion conductance and thus the depolarization of the cell from ion gradients across the cell membrane (Shevchenko et al., 2017). However, ion pumps with sufficiently fast pump rates not yet available in the optogenetic toolbox.

\section{Improving opsin expression:}

In order to facilitate stable light-induced neural responses, the opsin needs to be expressed at high levels and integrated into the plasma membrane. Previous studies have shown that trafficking of microbial opsins in mammalian neurons and proper membrane integration can be limited (Gradinaru et al., 2010; Keppeler et al., 2018; Wrobel et al., 2018). To improve opsin export from the endoplasmic reticulum, an export signal naturally occurring in inward rectifying potassium channels was added to the construct, resulting in enhanced export from the endoplasmic reticulum and improved opsin integration into the cell membrane (Gradinaru et al., 2010; Ma et al., 2001). A second trafficking signal derived from the same family of ion channels was reported to further increase localization of the expressed opsin to the cell membrane (Gradinaru et al., 2010; Stockklausner et al., 2001). In combination, these export and trafficking signals improved opsin targeting to the cell membrane not only in SGNs, but also in primary hippocampal cultures and HEK cells (Keppeler et al., 2018). In SGNs, improved membrane localization halved illumination intensity needed to evoke OABRs and increased the success rate of opsin expression after virus injection from $50 \%$ to $95 \%$ (Keppeler et al., 2018).

\section{Choosing the viral vector:}

Various viral vectors, including adeno-, adeno-associated-, lenti-, and rabies viruses - which all have their individual advantages and drawbacks - have been used for gene transfer to neural tissue. Out of this toolbox, AAVs are the most promising candidates for gene therapy, since they have an intrinsically high neural tropism, provide long-term protein expression at high levels, and have little potential for virus-related immune reactions or toxicity in the target tissue (Ahmed et al., 2017; Hudry and Vandenberghe, 2019; Lotfinia et al., 2019; Willett and Bennett, 2013). Various clinical trials for AAV-mediated gene therapy have been and are currently performed, including the treatment of retinal dysfunction (Askew et al., 2015; Hudry and Vandenberghe, 2019; Landegger et al., 2017; Lotfinia et al., 2019; Pan et al., 2017; Suzuki et al., 2017). Two recently approved clinical trials even involve optogenetic tools for vision restoration 
(NCT02556736 and NCT03326336). Furthermore, Luxturna - an AAV-mediated gene therapy for the treatment of vision loss due to Lebers congenital amaurosis - has recently been FDAapproved (Keeler and Flotte, 2019). No adverse reactions against AAVs were reported except mild, transient inflammatory responses. Transgene expression and vision restoration was stable for several years (Simonelli et al., 2010), and AAV-injection into the second eye has been approved and successfully performed (Bennett et al., 2012). For hearing restoration, gene therapies have not reached clinical trials yet. However, quite some studies have shown to restore auditory function in animals by AAV-mediated gene transfer (Ahmed et al., 2017; Al-Moyed et al., 2019; Askew et al., 2015; Landegger et al., 2017; Pan et al., 2017). In addition to naturally occurring AAVs, novel AAVs have been engineered recently in order to enhance their potential to transduce neural tissue. Both by targeted evolution (Deverman et al., 2016) and by in silico reconstruction (Chan et al., 2017; Zinn et al., 2015), transduction efficiency for various tissues, including hair cells and SGNs, could be greatly increased.

\section{Administration route:}

Several methods have been developed to optogenetically transduce the auditory nerve of different rodent species (Hernandez et al., 2014; Keppeler et al., 2018; Mager et al., 2018; Wrobel et al., 2018). In general, robust expression of optogenetic tools across all cochlear turns is desired for efficient optical coding, since OABR amplitudes have been shown to positively correlate with the amount of opsin expressing SGNs in the cochlea (Duarte et al., 2018; Hernandez et al., 2014; Wrobel et al., 2018). Furthermore, the method of virus administration should be minimally invasive and as atraumatic as possible. In this thesis, virus suspension has directly been injected into the modiolus, which houses the spiral ganglion, via a posterior tympanotomy (Wrobel et al., 2018). While this method of transduction resulted in homogeneous expression of opsins across the whole cochlea, the transduction efficiency was relatively low ( 30\%). Furthermore, only half of the injected animals were expressing the opsin, and SGN density was decreased by $\sim 25 \%$ across all cochlear turns, probably due to the pressure injection (since SGN reduction has also been observed in PBS-injected animals). In contrast, early postnatal injections of viral suspension into the cochlea of mice transduced $60-90 \%$ of SGNs, which were evenly distributed along the cochlear axis (Duarte et al., 2018; Keppeler et al., 2018; Mager et al., 2018). However, cochleae of early postnatal mice are weakly ossified, and the immune system of these animals not yet fully developed (Kraus and Aulbach-Kraus, 1981). In contrast, the human cochlea is highly ossified and almost fully developed at birth (Haith, 1986), raising the need for an efficient administration 
strategy tailored to the human inner ear. Future studies should thus include the development of an atraumatic method for reliable and efficient virus delivery to the auditory nerve in man.

\section{Evaluating the biosafety of cochlear optogenetics}

\section{Specificity of opsin expression:}

While the expression of optogenetic tools should be robust and efficient in the auditory nerve, opsin expression in off-target tissues beyond the inner ear should be excluded. Upon early postnatal virus injections into the mouse cochlea, substantial opsin expression has also been reported in the contralateral, non-injected ear (Keppeler et al., 2018; Mager et al., 2018). Most likely, viral particles reached the contralateral ear via the temporal bone marrow space or via the cochlear ducts, since the cochlea of early postnatal mice is not yet ossified(Kho et al., 2000). In contrast, opsin expression upon intramodiolar injections of virus suspension directly into the spiral ganglion of mature gerbils, as performed in this thesis, was restricted to SGNs in the injected ear (Wrobel et al., 2018). This raises hope that local virus application in the human cochlea will largely contribute to specific transduction, since the human inner ear is almost fully developed at birth (Haith, 1986). In addition to local virus administration, also the implementation of SGN-specific promoters will contribute to the specificity of opsin expression. Even though specific SGN promoters are not yet known, molecular profiling of SGNs is ongoing, and the results of these studies will likely enable the identification of adequate promoters to target the auditory nerve in future studies (Shrestha et al., 2018; Sun et al., 2018).

\section{Immune reactions:}

Before virally transducing a neural system and expressing alien proteins over extended periods of time in man, possible immune reactions of the host system should be carefully evaluated. Unfortunately, long-term studies of virus-mediated opsin-expression are relatively limited, since the application of optogenetic tools in neuroscience is a relatively novel method. Regarding the auditory system, two studies have reported the absence of cytotoxic effect in opsin-expressing tissue: While expression of f-Chrimson in the auditory nerve did not cause obvious cell loss over nine months (Mager et al., 2018), no cytotoxic effects have been identified in auditory brainstem neurons expressing ChR2 and halorhodopsin even for up to 18 months (Shimano et al., 2013). Additional studies, performed in the retina, reported the absence of cytotoxic effects in rodents 
up to one year after virus application (Bi et al., 2006; Busskamp et al., 2010; Doroudchi et al., 2011). In contrast, two studies have reported opsin aggregates due to overexpression, highlighting the importance to carefully evaluate any given construct for optogenetic transduction in preclinical studies, before considering its application in humans (Diester et al., 2011; Gradinaru et al., 2008). Besides harmful effects of the opsin itself, also possible immune responses against the viral vector must be taken into consideration. After retinal AAV-injections to cure vision loss with the FDA-approved gene therapy Luxturna, no virus-related harmful effects were observed except a mild and transient inflammatory response (Bennett et al., 2012; Simonelli et al., 2010). However, neutralizing antibodies, which might be present in the host organism, might hinder successful viral transduction of the target tissue. Antibodies might on the one hand result from natural AAV exposure before the gene therapy, and thus exclude some patients from the therapy (Hudry and Vandenberghe, 2019; Mendoza et al., 2017). On the other hand, neutralizing antibodies might be produced by the patient upon first administration of the virus, and consequently affect a second virus injection if needed (Hudry and Vandenberghe, 2019; Mendoza et al., 2017). Luckily, strategies to combat neutralizing antibodies are currently developed and include shielding of viral capsids, engineered capsids with reduced sensitivity to antibodies, or the use of empty capsids that bind neutralizing antibodies (Lotfinia et al., 2019; Mingozzi and High, 2013). Furthermore, local virus application might avoid global immune responses, since the cochlea is considered immune privileged due to the blood-labyrinth barrier. Taken together, currently available data of longitudinal studies in animals and humans raise the hope for AAVbased optogenetic transduction without severe damage to the target tissue. This is also highlighted by the fact that two clinical trials (NCT02556736 and NCT03326336) for optogeneticmediated vision restoration have recently been approved by the FDA. However, two preclinical studies on non-human primates (Hinderer et al., 2018; Hordeaux et al., 2018) and piglets (Hinderer et al., 2018) have reported adverse effects upon systemic application at high dosages. Consequently, careful evaluation of gene therapy safety for optogenetic hearing restoration including virus, gene dosage, administration route, promoter, opsins, and potential trafficking signals - are inevitable before considering human application. These studies should include nonhuman primates, since their immune system is more similar to humans than the immune system of rodents, which have so far been used to develop cochlear optogenetics.

\section{Phototoxicity:}

Another important aspect to evaluate before translating cochlear optogenetics is the cellular response to chronic illumination of neural tissue, since light interatcts with cells in various ways. 
Illumination over extended periods of time might have phototoxic effects, especially when using blue light, or lead to heating and changes in neural properties, such as long-term potentiation (Delbeke et al., 2017; Senova et al., 2017; Zhang and Oertner, 2007). So far, longitudinal data is not available for chronic illumination of neurons expression optogenetic tools. However, few studies have evaluated adverse light-induced effects, and irradiances up to $75 \mathrm{~mW} / \mathrm{mm}^{2}$ are generally considered to be safe for in vivo application of optogenetics (Cardin et al., 2010). One study used irradiances up to $600 \mathrm{~mW} / \mathrm{mm}^{2}$, and even though mild tissue heating $\left(0.1^{\circ} \mathrm{C}\right.$ with blue light, $0.3^{\circ} \mathrm{C}$ with red light) has been observed upon illumination at $200 \mathrm{~mW} / \mathrm{mm}^{2}$, neither phototoxic effects nor apoptosis or even cell loss have been observed upon illumination with up to $600 \mathrm{~mW} / \mathrm{mm}^{2}$ (Senova et al., 2017). Even though the safety limits of chronic SGN illumination must still be defined, they will strongly depend on the action spectrum of the opsin of choice. Safety limits defined for the retina (European commission directive 2006/25/EC, and International Commission on Nonionizing Radiation Protection) are three orders of magnitude lower for blue light as for orange light, since blue light has a higher potential of phototoxicity - which is probably also true for cells in the inner ear (Duebel et al., 2015; Sengupta et al., 2016). Thus, a red-shifted action spectrum of opsins for hearing restoration is desirable, since it would increase the safety for patients substantially.

\section{$\underline{\text { Remaining technological developments }}$}

\section{Medical device:}

Even though first optical cochlear implants have been developed in the previous years, the need for optimization still remains (Balster et al., 2014; Goßler et al., 2014; Klein et al., 2018; Schwaerzle et al., 2016; Xu et al., 2018): To take maximal advantage of the improved spectral selectivity of optical sound encoding, the number of independent light sources of a future oCl needs to substantially surpass the number of channels employed by eCls. State of the art eCls house between 12-24 electrodes, of which typically less than ten are independent (i.e. are actually available for sound encoding by the coding strategy and processor)(Friesen et al., 2001). As the frequency resolution of optical sound encoding has been estimated to be at least two-fold higher than the resolution of electrical sound encoding (which might even be an underestimation), oCls should contain double as much stimulation sites at a minimum, ideally even more, which would be in the range of 50 to more than 100 light sources (Dieter et al., 2019). 
Even though one of the recently engineered oCls fits these requirements, functionality and longterm stability of this implant in chronic experiments need to be demonstrated (Klein et al., 2018). Regarding implant stability, encapsulation of the oCl will be of critical importance, since this barrier protects both the inner ear and the optoelectronic device. By physically separating the light source from biological fluids and tissue, the barrier provided by the encapsulation avoids leakage of alien molecules from the optoelectronic implant. Conversely, optoelectronics of the implant are protected from intracochlear fluids, which might lead to emitter damage or shortcutting of the current supply. Hermetically sealed light sources in a passive oCl easily meet the stability requirements, since the light sources are heavily protected and might even be exchanged in case of failure. However, properly encapsulated active optoelectronics in the cochlea might also be feasible, since the life expectancy of state of the art LEDs is reported to be as high as 11 years, which, when driven with a lower duty cycle, promise optical auditory nerve stimulation over decades (Laubsch et al., 2010). In any case, the long-term stability of eCls, which typically is stable over several decades, is a tough benchmark for future oCls to meet. To guarantee more safety to the patient and maximize the possibilities of successful hearing restoration, hybrid implants could be employed for first clinical trials. Combining optical emitters with electrode contacts in one implant, the $\mathrm{Cl}$ would offer increased coding capabilities due to optical sound encoding, but still provide the possibility of conventional electric stimulation as a backup. Should optical sound encoding fail, state of the art electrical sound encoding would still be possible.

\section{Coding strategy:}

Besides gene therapy and innovative multi-channel stimulators, hearing restoration by optogenetics also requires a tailor-made strategy for optical sound encoding. This strategy depends both on the channel kinetics of the implemented opsin, and on the number of light emitters on the oCl. Optical sound encoding will take advantage of the increased number of stimulation channels, which will independently deliver pulses of varying energy and repetition rate. The strength of neural responses increases with increasing optical energy which will thus be implemented for the coding of sound intensity (Dieter et al., 2019). Both illumination intensity and pulse duration determine the energy of a pulse, and hence the coding strategy must balance intensity and pulse duration in a way that avoids phototoxic effects at high intensities but also enables sufficiently short pulse durations to facilitate stimulation at high repetition rates. Based on experimental data, pulse durations between 0.1-1 ms seem reasonable, since no activity could be evoked with shorter pulses, and oABR amplitudes decreased for pulses longer than $1 \mathrm{~ms}$ 
(probably due to channel inactivation) (Keppeler et al., 2018; Mager et al., 2018; Wrobel et al., 2018). However, by taking advantage of temporal integration of photocurrents, it might be possible to compose stimulation from pulses below $100 \mu$ s if stimulation rates are sufficiently high. This might have the advantage to avoid channel interaction, as inspired by the continuous interleaved sampling strategy employed in modern eCl coding (Wilson et al., 1991). In contrast to the increased amount of independent stimulation channels, stimulation rates of optogenetic sound encoding will most likely be lower than for electrical sound encoding. Current eCls employ stimulation rates reaching from few hundred up to $3,000 \mathrm{~Hz}$ (Zeng et al., 2008). This is due to the fact that spike jitter in response to electrical stimulation is very low, and overly synchronized activation of large SGN populations seems to reduce speech understanding (Rubinstein, 2004). Increasing the stimulation rates beyond the maximum firing rate of auditory neurons induces jitter to neural responses (Litvak et al., 2003), which improves the coding of temporal fine structure, dynamic range, and inter-aural time differences in bilaterally implanted patients (Goupell et al., 2009; Hong et al., 2003; Rubinstein, 2004). As opposed to electrical stimulation, for which the jitter of auditory nerve firing is as low as 10-30 $\mu$ s (van den Honert and Stypulkowski, 1984), opsin-dependent spike jitter in the range of 200-300 $\mu$ s has been reported for optogenetic SGN activation (Hernandez et al., 2014; Keppeler et al., 2018; Mager et al., 2018; Wrobel et al., 2018). Because of this larger jitter, lower stimulation rates already de-synchronize the auditory nerve sufficiently in response to optical stimulation, and hence allow for decreasing stimulation rates. Furthermore, studies have shown that the enormous increase in stimulation rates in eCls does not substantially improve speech recognition, and vocoded noise stimuli which were modulated with the envelope of human speech signals did not increase word recognition when modulated with frequencies higher than few hundred $\mathrm{Hz}$ (Shannon et al., 1995, 2011). Thus, optical sound encoding with stimulation rates of few hundreds of $\mathrm{Hz}$ should be sufficient to intelligibly encode human speech. Nevertheless, the development of a coding strategy for oCls will largely depend on the final choice of opsin and stimulator design.

\section{Translation to non-human primates:}

Hearing restoration by optogenetic cochlear implants combines gene therapy in the auditory nerve with the implantation of multi-channel optical stimulators and real-time sound processors with a coding strategy optimized for opsin kinetics and channel number of future molecular tools and implantable devices. Before clinical translation, these different approaches should be integrated and thoroughly tested in non-human primates. Regarding anatomy, physiology, and 
behavior, non-human primates are much more similar to humans than mice are, and thus provide a valuable model for preclinical studies of biomedical developments (Ahmed et al., 2017; Sengupta et al., 2016). In these studies, efficiency and safety of viral vectors and molecular tools should be demonstrated, and the insights from rodent work should be corroborated. Furthermore, functionality and long-term stability of multi-channel oCls must be demonstrated. The efficiency of optical sound encoding via multi-channel implants driven by sound processors with a tailor-made coding strategy must be benchmarked against state of the art electrical sound encoding. A suitable model for non-human primate studies would be the common marmoset: Marmosets have a rich vocal repertoire, a comparable hearing range to humans, and a similar cortex structure. Due to these aspects, marmosets received increasing attention as a model in auditory neuroscience (Eliades and Miller, 2017), and more recently became an important model for cochlear implant research (Johnson et al., 2016). Furthermore, marmosets can be trained in complex behavioral tasks on stimulus detection and discrimination, both using acoustic (Remington et al., 2012) and electrical sound encoding (Johnson et al., 2016). A comparison of behaviorally relevant artificial sound encoding by electrical and optical $\mathrm{Cls}$ in marmosets would thus depict the ideal model for preclinical benchmarking of oCl technology. These experiments should subsequently be complemented by post-mortem studies, which screen for unwanted side effects of optogenetic gene therapy such as opsin expression beyond the target tissues, cytotoxic or phototoxic effects and local as well as global immune reactions, in order to guarantee the highest level of safety for clinical translation. Inspired by the development of optogenetics for vision restoration, longitudinal studies in non-human primates could be complemented by experiments involving human tissue or even inner ear organoids, to optimally prepare the way for clinical translation of cochlear optogenetics (Garita-Hernandez et al., 2018; Koehler et al., 2017; Landegger et al., 2017; Sengupta et al., 2016). 


\subsection{Cochlear optogenetics as a tool for auditory neuroscience}

Besides promising improved capabilities of artificial sound encoding for hearing restoration, cochlear optogenetics will help to innovatively address open questions in the field of auditory neuroscience. Acoustic sound encoding by the organ of Corti is inherently entangled with the micromechanics of the cochlea: For example, the amplitude of the cochlear traveling wave, which is thought to mainly encode sound intensity, to some degree determines the location at which the travelling wave maximally deflects the basilar membrane, resulting in an intensity-dependent tonotopic shift (Johnstone et al., 1986). Hence, the place-frequency code of the cochlea to some degree depends on sound intensity, both on a physiological and on a psychophysical level (Tao et al., 2017). The travelling wave also deflects the basilar membrane surrounding the region of maximum deflection in a stimulus-level dependent manner, and furthermore it resonates for some time after stimulus-off (especially after short, transient stimuli, such as acoustic clicks), limiting the precision of acoustic hair cell activation both in space and in time (Chatterjee and Zwislocki, 1998; Johnstone and Boyle, 1967; Zhang et al., 2018a). In addition, the pitch of low frequency sounds is not only coded by tonotopic activation of the auditory nerve, but also by the timing of action potentials (Joris and Smith, 2008). This temporal code for sound frequency is inseparable from the place-frequency code that frequency coding mainly relies on. In contrast to acoustic stimulation, spatially confined optical stimulation would enable activation of SGNs along the tonotopic axis with high spectral (and potentially intensity) selectivity, while bypassing the implications of cochlear micromechanics. Optogenetic SGN activation would thus allow for stimulation of the auditory nerve with arbitrary stimulation patterns, de-coupling spectral, temporal and intensity information of the stimulus. These degrees of freedom in stimulus design by optogenetics would offer the possibility to address unanswered questions in auditory research that were previously limited by cochlear micromechanics: For example, it has been shown that the pitch of a fundamental frequency in a harmonic complex is perceived both by animals (Heffner and Whitfield, 1976; Tomlinson and Schwarz, 1988) and by humans (Fujioka et al., 2003) even if this fundamental frequency has been removed from the original sound (Wang and Walker, 2012). However, it is not known if the neural correlate of missing fundamental perception originates in the cochlea, or rather emerges during central auditory processing. Also, studies on the origin of spectrotemporal integration of periodic sounds, such as amplitude-modulated pure tones, have been limited by the inherent connection of spectral and temporal properties of acoustic stimuli (Wang and Walker, 2012). Optogenetic stimulation of the auditory nerve with high spectro-temporal precision might provide insight in these processes, which are thought to 
contribute to the perception and segregation of auditory objects and scene analysis. Besides investigating the processing mechanisms of complex sounds, cochlear optogenetics might also contribute to further decipher the role of SGN subtypes in auditory coding: type I SGNs, which pass information from inner hair cells to the auditory brainstem, have been categorized into three physiologically distinct subtypes, based on their thresholds and firing properties (Kiang et al., 1965; Liberman, 1978). More recently, molecular correlates of these subtypes have been described (Shrestha et al., 2018). Implementation of subtype-specific promoters and selective expression of opsins in these subtypes would enable independent activation of SGN populations with different properties and most likely function, and could contribute to a deeper understanding of sound encoding. Thus, besides promising improved capabilities for hearing restoration, cochlear optogenetics would also provide an innovative tool for investigating auditory function. 


\section{Bibliography}

Ahmed, H., Shubina-Oleinik, O., and Holt, J.R. (2017). Emerging Gene Therapies for Genetic Hearing Loss. J. Assoc. Res. Otolaryngol. 18, 649-670.

Akil, O., Seal, R.P., Burke, K., Wang, C., Alemi, A., During, M., Edwards, R.H., and Lustig, L.R. (2012). Restoration of Hearing in the VGLUT3 Knockout Mouse Using Virally Mediated Gene Therapy. Neuron 75, 283-293.

Allitt, B.J., Morgan, S.J., Bell, S., Nayagam, D.A.X., Arhatari, B., Clark, G.M., and Paolini, A.G. (2012). Midbrain responses to micro-stimulation of the cochlea using high density thin-film arrays. Hear. Res. 287, 30-42.

Allitt, B.J., Harris, A.R., Morgan, S.J., Clark, G.M., and Paolini, A.G. (2016). Thin-film microelectrode stimulation of the cochlea in rats exposed to aminoglycoside induced hearing loss. Hear. Res. 331, 13-26.

Al-Moyed, H., Cepeda, A.P., Jung, S., Moser, T., Kügler, S., and Reisinger, E. (2019). A dual-AAV approach restores fast exocytosis and partially rescues auditory function in deaf otoferlin knockout mice. EMBO Mol. Med. 11, e9396.

Alt, M.T., Fiedler, E., Rudmann, L., Ordonez, J.S., Ruther, P., and Stieglitz, T. (2017). Let There Be Light - Optoprobes for Neural Implants. Proc. IEEE 105, 101-138.

Arenkiel, B.R., Peca, J., Davison, I.G., Feliciano, C., Deisseroth, K., Augustine, G.J., Ehlers, M.D., and Feng, G. (2007). In Vivo Light-Induced Activation of Neural Circuitry in Transgenic Mice Expressing Channelrhodopsin-2. Neuron 54, 205-218.

Askew, C., Rochat, C., Pan, B., Asai, Y., Ahmed, H., Child, E., Schneider, B.L., Aebischer, P., and Holt, J.R. (2015). Tmc gene therapy restores auditory function in deaf mice. Sci. Transl. Med. 7, 295ra108-295ra108.

Balster, S., Wenzel, G.I., Warnecke, A., Steffens, M., Rettenmaier, A., Zhang, K., Lenarz, T., and Reuter, G. (2014). Optical cochlear implant: evaluation of insertion forces of optical fibres in a cochlear model and of traumata in human temporal bones. Biomed. Tech. Eng. 59, 19-28.

Bennett, J., Ashtari, M., Wellman, J., Marshall, K.A., Cyckowski, L.L., Chung, D.C., McCague, S., Pierce, E.A., Chen, Y., Bennicelli, J.L., et al. (2012). AAV2 gene therapy readministration in three adults with congenital blindness. Sci. Transl. Med. 4, 120ra15.

Berndt, A., Yizhar, O., Gunaydin, L.A., Hegemann, P., and Deisseroth, K. (2008). Bi-stable neural state switches. Nat. Neurosci. 12, 229-234.

Bi, A., Cui, J., Ma, Y.-P., Olshevskaya, E., Pu, M., Dizhoor, A.M., and Pan, Z.-H. (2006). Ectopic Expression of a Microbial-Type Rhodopsin Restores Visual Responses in Mice with Photoreceptor Degeneration. Neuron 50, 23-33.

Boyden, E.S., Zhang, F., Bamberg, E., Nagel, G., and Deisseroth, K. (2005). Millisecond-timescale, genetically targeted optical control of neural activity. Nat Neurosci 8, 1263-1268. 
Busskamp, V., Duebel, J., Balya, D., Fradot, M., Viney, T.J., Siegert, S., Groner, A.C., Cabuy, E., Forster, V., Seeliger, M., et al. (2010). Genetic Reactivation of Cone Photoreceptors Restores Visual Responses in Retinitis Pigmentosa. Science 329, 413-417.

Cardin, J.A., Carlén, M., Meletis, K., Knoblich, U., Zhang, F., Deisseroth, K., Tsai, L.-H., and Moore, C.I. (2010). Targeted optogenetic stimulation and recording of neurons in vivo using cell-typespecific expression of Channelrhodopsin-2. Nat. Protoc. 5, 247-254.

Chan, K.Y., Jang, M.J., Yoo, B.B., Greenbaum, A., Ravi, N., Wu, W.-L., Sánchez-Guardado, L., Lois, C., Mazmanian, S.K., Deverman, B.E., et al. (2017). Engineered AAVs for efficient noninvasive gene delivery to the central and peripheral nervous systems. Nat. Neurosci. 20, 1172-1179.

Chang, Q., Tang, W., Kim, Y., and Lin, X. (2015). Timed conditional null of connexin26 in mice reveals temporary requirements of connexin 26 in key cochlear developmental events before the onset of hearing. Neurobiol. Dis. 73, 418-427.

Chatterjee, M., and Zwislocki, J.J. (1998). Cochlear mechanisms of frequency and intensity coding. II. Dynamic range and the code for loudness. Hear. Res. 124, 170-181.

Chen, W., Jongkamonwiwat, N., Abbas, L., Eshtan, S.J., Johnson, S.L., Kuhn, S., Milo, M., Thurlow, J.K., Andrews, P.W., Marcotti, W., et al. (2012). Restoration of auditory evoked responses by human ES-cell-derived otic progenitors. Nature 490, 278-282.

Choi, B.Y., Kim, H.-M., Ito, T., Lee, K.-Y., Li, X., Monahan, K., Wen, Y., Wilson, E., Kurima, K., Saunders, T.L., et al. (2011). Mouse model of enlarged vestibular aqueducts defines temporal requirement of Slc26a4 expression for hearing acquisition. J. Clin. Invest. 121, 4516-4525.

Corrales, C.E., Pan, L., Li, H., Liberman, M.C., Heller, S., and Edge, A.S.B. (2006). Engraftment and differentiation of embryonic stem cell-derived neural progenitor cells in the cochlear nerve trunk: growth of processes into the organ of Corti. J. Neurobiol. 66, 1489-1500.

Deisseroth, K., and Hegemann, P. (2017). The form and function of channelrhodopsin. Science 357.

Delbeke, J., Hoffman, L., Mols, K., Braeken, D., and Prodanov, D. (2017). And Then There Was Light: Perspectives of Optogenetics for Deep Brain Stimulation and Neuromodulation. Front. Neurosci. 11, 663.

Deverman, B.E., Pravdo, P.L., Simpson, B.P., Kumar, S.R., Chan, K.Y., Banerjee, A., Wu, W.-L., Yang, B., Huber, N., Pasca, S.P., et al. (2016). Cre-dependent selection yields AAV variants for widespread gene transfer to the adult brain. Nat. Biotechnol. 34, 204-209.

Diester, I., Kaufman, M.T., Mogri, M., Pashaie, R., Goo, W., Yizhar, O., Ramakrishnan, C., Deisseroth, K., and Shenoy, K.V. (2011). An optogenetic toolbox designed for primates. Nat. Neurosci. 14, 387-397.

Dieter, A., Duque-Afonso, C.J., Rankovic, V., Jeschke, M., and Moser, T. (2019). Near physiological spectral selectivity of cochlear optogenetics. Nat. Commun. 10, 1962.

Dombrowski, T., Rankovic, V., and Moser, T. (2018). Toward the Optical Cochlear Implant. Cold Spring Harb. Perspect. Med. a033225. 
Donaldson, G.S., Kreft, H.A., and Litvak, L. (2005). Place-pitch discrimination of single- versus dualelectrode stimuli by cochlear implant users (L). J. Acoust. Soc. Am. 118, 623-626.

Doroudchi, M.M., Greenberg, K.P., Liu, J., Silka, K.A., Boyden, E.S., Lockridge, J.A., Arman, A.C., Janani, R., Boye, S.E., Boye, S.L., et al. (2011). Virally delivered Channelrhodopsin-2 Safely and Effectively Restores Visual Function in Multiple Mouse Models of Blindness. Mol. Ther. 19, 12201229.

Dougherty, W., and Kesser, B.W. (2015). Management of Conductive Hearing Loss in Children. Otolaryngol. Clin. North Am. 48, 955-974.

Duarte, M.J., Kanumuri, V.V., Landegger, L.D., Tarabichi, O., Sinha, S., Meng, X., Hight, A.E., Kozin, E.D., Stankovic, K.M., Brown, M.C., et al. (2018). Ancestral Adeno-Associated Virus Vector Delivery of Opsins to Spiral Ganglion Neurons: Implications for Optogenetic Cochlear Implants. Mol. Ther. 26, 1931-1939.

Duebel, J., Marazova, K., and Sahel, J.-A. (2015). Optogenetics. Curr. Opin. Ophthalmol. 26, 226232.

Eliades, S.J., and Miller, C.T. (2017). Marmoset vocal communication: Behavior and neurobiology. Dev. Neurobiol. 77, 286-299.

Fenno, L., Yizhar, O., and Deisseroth, K. (2011). The development and application of optogenetics. Annu. Rev. Neurosci. 34, 389-412.

Formby, C. (1986). Frequency and rate discrimination by Menière patients. Audiol. Off. Organ Int. Soc. Audiol. 25, 10-18.

Friesen, L.M., Shannon, R.V., Baskent, D., and Wang, X. (2001). Speech recognition in noise as a function of the number of spectral channels: Comparison of acoustic hearing and cochlear implants. J. Acoust. Soc. Am. 110, 1150.

Fujioka, T., Ross, B., Okamoto, H., Takeshima, Y., Kakigi, R., and Pantev, C. (2003). Tonotopic representation of missing fundamental complex sounds in the human auditory cortex. Eur. J. Neurosci. 18, 432-440.

Garita-Hernandez, M., Guibbal, L., Toualbi, L., Routet, F., Chaffiol, A., Winckler, C., Harinquet, M., Robert, C., Fouquet, S., Bellow, S., et al. (2018). Optogenetic Light Sensors in Human Retinal Organoids. Front. Neurosci. 12.

Gates, G.A. (2012). Central presbycusis: an emerging view. Otolaryngol.--Head Neck Surg. Off. J. Am. Acad. Otolaryngol.-Head Neck Surg. 147, 1-2.

Gates, G.A., Cobb, J.L., Linn, R.T., Rees, T., Wolf, P.A., and D'Agostino, R.B. (1996). Central auditory dysfunction, cognitive dysfunction, and dementia in older people. Arch. Otolaryngol. Head Neck Surg. 122, 161-167.

Géléoc, G.S.G., and Holt, J.R. (2014). Sound strategies for hearing restoration. Science 344, 1241062. 
George, S.S., Shivdasani, M.N., Wise, A.K., Shepherd, R.K., and Fallon, J.B. (2015). Electrophysiological channel interactions using focused multipolar stimulation for cochlear implants. J. Neural Eng. 12, 066005.

Goßler, C., Bierbrauer, C., Moser, R., Kunzer, M., Holc, K., Pletschen, W., Köhler, K., Wagner, J., Schwaerzle, M., Ruther, P., et al. (2014). GaN-based micro-LED arrays on flexible substrates for optical cochlear implants. J. Phys. Appl. Phys. 47, 205401.

Goupell, M.J., Laback, B., and Majdak, P. (2009). Enhancing sensitivity to interaural time differences at high modulation rates by introducing temporal jitter. J. Acoust. Soc. Am. 126, 25112521.

Gradinaru, V., Thompson, K.R., and Deisseroth, K. (2008). eNpHR: a Natronomonas halorhodopsin enhanced for optogenetic applications. Brain Cell Biol. 36, 129-139.

Gradinaru, V., Zhang, F., Ramakrishnan, C., Mattis, J., Prakash, R., Diester, I., Goshen, I., Thompson, K.R., and Deisseroth, K. (2010). Molecular and Cellular Approaches for Diversifying and Extending Optogenetics. Cell 141, 154-165.

Gunaydin, L.A., Yizhar, O., Berndt, A., Sohal, V.S., Deisseroth, K., and Hegemann, P. (2010). Ultrafast optogenetic control. Nat. Neurosci. 13, 387-392.

Guo, W., Hight, A.E., Chen, J.X., Klapoetke, N.C., Hancock, K.E., Shinn-Cunningham, B.G., Boyden, E.S., Lee, D.J., and Polley, D.B. (2015). Hearing the light: neural and perceptual encoding of optogenetic stimulation in the central auditory pathway. Sci. Rep. 5.

Haith, M.M. (1986). Sensory and perceptual processes in early infancy. J. Pediatr. 109, 158-171.

Heffner, H., and Whitfield, I.C. (1976). Perception of the missing fundamental by cats. J. Acoust. Soc. Am. 59, 915-919.

Heil, P., and Peterson, A.J. (2015). Basic response properties of auditory nerve fibers: a review. Cell Tissue Res. 361, 129-158.

Henry, K.R. (1979). Auditory brainstem volume-conducted responses: origins in the laboratory mouse. J. Am. Aud. Soc. 4, 173-178.

Hernandez, V.H., Gehrt, A., Reuter, K., Jing, Z., Jeschke, M., Mendoza Schulz, A., Hoch, G., Bartels, M., Vogt, G., Garnham, C.W., et al. (2014). Optogenetic stimulation of the auditory pathway. J. Clin. Invest. 124, 1114-1129.

Hinderer, C., Katz, N., Buza, E.L., Dyer, C., Goode, T., Bell, P., Richman, L.K., and Wilson, J.M. (2018). Severe Toxicity in Nonhuman Primates and Piglets Following High-Dose Intravenous Administration of an Adeno-Associated Virus Vector Expressing Human SMN. Hum. Gene Ther. 29, 285-298.

Hofherr, A. (2005). Selective Golgi export of Kir2.1 controls the stoichiometry of functional Kir2.x channel heteromers. J. Cell Sci. 118, 1935-1943.

van den Honert, C., and Stypulkowski, P.H. (1984). Physiological properties of the electrically stimulated auditory nerve. II. Single fiber recordings. Hear. Res. 14, 225-243. 
Hong, R.S., Rubinstein, J.T., Wehner, D., and Horn, D. (2003). Dynamic range enhancement for cochlear implants. Otol. Neurotol. Off. Publ. Am. Otol. Soc. Am. Neurotol. Soc. Eur. Acad. Otol. Neurotol. 24, 590-595.

Hordeaux, J., Wang, Q., Katz, N., Buza, E.L., Bell, P., and Wilson, J.M. (2018). The Neurotropic Properties of AAV-PHP.B Are Limited to C57BL/6J Mice. Mol. Ther. J. Am. Soc. Gene Ther. 26, 664668.

Hudry, E., and Vandenberghe, L.H. (2019). Therapeutic AAV Gene Transfer to the Nervous System: A Clinical Reality. Neuron 101, 839-862.

Husain, F.T., Carpenter-Thompson, J.R., and Schmidt, S.A. (2014). The effect of mild-to-moderate hearing loss on auditory and emotion processing networks. Front. Syst. Neurosci. 8, 10.

Irvine, D.R.F. (2018). Plasticity in the auditory system. Hear. Res. 362, 61-73.

Izzo, A.D., Suh, E., Pathria, J., Walsh, J.T., Whitlon, D.S., and Richter, C.-P. (2007). Selectivity of neural stimulation in the auditory system: a comparison of optic and electric stimuli. J. Biomed. Opt. 12, 021008.

Jacques, S.L. (2013). Optical properties of biological tissues: a review. Phys. Med. Biol. 58, R37R61.

Jeschke, M., and Moser, T. (2015). Considering optogenetic stimulation for cochlear implants. Hear. Res. 322, 224-234.

Jewett, S.A., Makowski, M.S., Andrews, B., Manfra, M.J., and Ivanisevic, A. (2012). Gallium nitride is biocompatible and non-toxic before and after functionalization with peptides. Acta Biomater. 8 , 728-733.

Johnson, L.A., Della Santina, C.C., and Wang, X. (2016). Selective Neuronal Activation by Cochlear Implant Stimulation in Auditory Cortex of Awake Primate. J. Neurosci. 36, 12468-12484.

Johnstone, B.M., and Boyle, A.J. (1967). Basilar membrane vibration examined with the Mössbauer technique. Science 158, 389-390.

Johnstone, B.M., Patuzzi, R., and Yates, G.K. (1986). Basilar membrane measurements and the travelling wave. Hear. Res. 22, 147-153.

Joris, P.X., and Smith, P.H. (2008). The volley theory and the spherical cell puzzle. Neuroscience $154,65-76$.

José, M.R., Mondelli, M.F.C.G., Feniman, M.R., and Lopes-Herrera, S.A. (2014). Language disorders in children with unilateral hearing loss: a systematic review. Int. Arch. Otorhinolaryngol. 18, 198203.

Kallweit, N., Baumhoff, P., Krueger, A., Tinne, N., Kral, A., Ripken, T., and Maier, H. (2016). Optoacoustic effect is responsible for laser-induced cochlear responses. Sci. Rep. 6.

Keeler, A.M., and Flotte, T.R. (2019). Recombinant Adeno-Associated Virus Gene Therapy in Light of Luxturna (and Zolgensma and Glybera): Where Are We, and How Did We Get Here? Annu. Rev. Virol. 
Keppeler, D., Merino, C., and Lopez, D. (2018). Ultrafast optogenetic stimulation of the auditory pathway by targeting-optimized Chronos. EMBO J.

Kerstein, R.L., Lister, T., and Cole, R. (2014). Laser therapy and photosensitive medication: a review of the evidence. Lasers Med. Sci. 29, 1449-1452.

Kho, S.T., Pettis, R.M., Mhatre, A.N., and Lalwani, A.K. (2000). Safety of adeno-associated virus as cochlear gene transfer vector: analysis of distant spread beyond injected cochleae. Mol. Ther. J. Am. Soc. Gene Ther. 2, 368-373.

Khouri, L., and Nelken, I. (2015). Detecting the unexpected. Curr. Opin. Neurobiol. 35, 142-147.

Kiang, N.Y.S., Watanabe, T., Thomas, E.C., and Clark, L.F. (1965). Discharge Patterns of Single Fibers in the Cat's Auditory Nerve (Cambridge, Massachusetts: MIT Press).

Klapoetke, N.C., Murata, Y., Kim, S.S., Pulver, S.R., Birdsey-Benson, A., Cho, Y.K., Morimoto, T.K., Chuong, A.S., Carpenter, E.J., Tian, Z., et al. (2014). Independent optical excitation of distinct neural populations. Nat. Methods 11, 338-346.

Klein, E., Gossler, C., Paul, O., and Ruther, P. (2018). High-Density $\mu$ LED-Based Optical Cochlear Implant With Improved Thermomechanical Behavior. Front. Neurosci. 12.

Klein, E., Kaku, Y., Paul, O., and Ruther, P. (2019). Flexible $\mu$ LED-based optogenetic tool with integrated $\mu$-lens array and conical concentrators providing light extraction improvements above 80\%. IEEE MEMS 632-635.

Kleinlogel, S., Feldbauer, K., Dempski, R.E., Fotis, H., Wood, P.G., Bamann, C., and Bamberg, E. (2011). Ultra light-sensitive and fast neuronal activation with the $\mathrm{Ca}^{2}+$-permeable channelrhodopsin CatCh. Nat. Neurosci. 14, 513-518.

Knöpfel, T., Lin, M.Z., Levskaya, A., Tian, L., Lin, J.Y., and Boyden, E.S. (2010). Toward the second generation of optogenetic tools. J. Neurosci. Off. J. Soc. Neurosci. 30, 14998-15004.

Koehler, K.R., Mikosz, A.M., Molosh, A.I., Patel, D., and Hashino, E. (2013). Generation of inner ear sensory epithelia from pluripotent stem cells in 3D culture. Nature 500, 217-221.

Koehler, K.R., Nie, J., Longworth-Mills, E., Liu, X.-P., Lee, J., Holt, J.R., and Hashino, E. (2017). Generation of inner ear organoids containing functional hair cells from human pluripotent stem cells. Nat. Biotechnol. 35, 583-589.

Kohlberg, G., Spitzer, J.B., Mancuso, D., and Lalwani, A.K. (2014). Does cochlear implantation restore music appreciation?: Music Appreciation With Cl. The Laryngoscope 124, 587-588.

Kral, A. (2013). Auditory critical periods: a review from system's perspective. Neuroscience 247, 117-133.

Kral, A., and Sharma, A. (2012). Developmental neuroplasticity after cochlear implantation. Trends Neurosci. 35, 111-122.

Kral, A., Hartmann, R., Mortazavi, D., and Klinke, R. (1998). Spatial resolution of cochlear implants: the electrical field and excitation of auditory afferents. Hear. Res. 121, 11-28. 
Kraus, H.J., and Aulbach-Kraus, K. (1981). Morphological changes in the cochlea of the mouse after the onset of hearing. Hear. Res. 4, 89-102.

Land, R., Burghard, A., and Kral, A. (2016). The contribution of inferior colliculus activity to the auditory brainstem response (ABR) in mice. Hear. Res. 341, 109-118.

Landegger, L.D., Pan, B., Askew, C., Wassmer, S.J., Gluck, S.D., Galvin, A., Taylor, R., Forge, A., Stankovic, K.M., Holt, J.R., et al. (2017). A synthetic AAV vector enables safe and efficient gene transfer to the mammalian inner ear. Nat. Biotechnol. 35, 280-284.

Laubsch, A., Sabathil, M., Baur, J., Peter, M., and Hahn, B. (2010). High-Power and High-Efficiency InGaN-Based Light Emitters. IEEE Trans. Electron Devices 57, 79-87.

Lee, J.W., and Bance, M.L. (2018). Hearing loss. Pract. Neurol.

Lenarz, T. (2018). Cochlear implant - state of the art. GMS Curr. Top. Otorhinolaryngol. Head Neck Surg. 16, Doc04.

Lentz, J.J., Jodelka, F.M., Hinrich, A.J., McCaffrey, K.E., Farris, H.E., Spalitta, M.J., Bazan, N.G., Duelli, D.M., Rigo, F., and Hastings, M.L. (2013). Rescue of hearing and vestibular function by antisense oligonucleotides in a mouse model of human deafness. Nat. Med. 19, 345-350.

Li, H., Roblin, G., Liu, H., and Heller, S. (2003). Generation of hair cells by stepwise differentiation of embryonic stem cells. Proc. Natl. Acad. Sci. U. S. A. 100, 13495-13500.

Liang, C., Earl, B., Thompson, I., Whitaker, K., Cahn, S., Xiang, J., Fu, Q.-J., and Zhang, F. (2016). Musicians Are Better than Non-musicians in Frequency Change Detection: Behavioral and Electrophysiological Evidence. Front. Neurosci. 10, 464.

Liberman, M.C. (1978). Auditory-nerve response from cats raised in a low-noise chamber. J. Acoust. Soc. Am. 63, 442-455.

Litvak, L.M., Smith, Z.M., Delgutte, B., and Eddington, D.K. (2003). Desynchronization of electrically evoked auditory-nerve activity by high-frequency pulse trains of long duration. J. Acoust. Soc. Am. 114, 2066-2078.

Lotfinia, M., Abdollahpour-Alitappeh, M., Hatami, B., Zali, M.R., and Karimipoor, M. (2019). Adeno-associated virus as a gene therapy vector: strategies to neutralize the neutralizing antibodies. Clin. Exp. Med. 19, 289-298.

Ma, D., Zerangue, N., Lin, Y.F., Collins, A., Yu, M., Jan, Y.N., and Jan, L.Y. (2001). Role of ER export signals in controlling surface potassium channel numbers. Science 291, 316-319.

Mager, T., Lopez de la Morena, D., Senn, V., Schlotte, J., D Errico, A., Feldbauer, K., Wrobel, C., Jung, S., Bodensiek, K., Rankovic, V., et al. (2018). High frequency neural spiking and auditory signaling by ultrafast red-shifted optogenetics. Nat. Commun. 9, 1750.

Matic, A.I., Robinson, A.M., Young, H.K., Badofsky, B., Rajguru, S.M., Stock, S., and Richter, C.-P. (2013). Behavioral and Electrophysiological Responses Evoked by Chronic Infrared Neural Stimulation of the Cochlea. PLoS ONE 8, e58189. 
Mendoza, S.D., El-Shamayleh, Y., and Horwitz, G.D. (2017). AAV-mediated delivery of optogenetic constructs to the macaque brain triggers humoral immune responses. J. Neurophysiol. 117, 20042013.

Meurmann, O.H. (1954). The difference limen of frequency in tests of auditory function. Acta OtoLaryngol. Suppl. 118, 144-155.

Middlebrooks, J.C., and Snyder, R.L. (2007). Auditory prosthesis with a penetrating nerve array. J. Assoc. Res. Otolaryngol. JARO 8, 258-279.

Middlebrooks, J.C., Bierer, J.A., and Snyder, R.L. (2005). Cochlear implants: the view from the brain. Curr. Opin. Neurobiol. 15, 488-493.

Miller, C.A., Abbas, P.J., Robinson, B.K., Nourski, K.V., Zhang, F., and Jeng, F.-C. (2006). Electrical Excitation of the Acoustically Sensitive Auditory Nerve: Single-Fiber Responses to Electric Pulse Trains. J. Assoc. Res. Otolaryngol. 7, 195-210.

Mingozzi, F., and High, K.A. (2013). Immune responses to AAV vectors: overcoming barriers to successful gene therapy. Blood 122, 23-36.

Moore, D.R., and Shannon, R.V. (2009). Beyond cochlear implants: awakening the deafened brain. Nat. Neurosci. 12, 686-691.

Moser, T. (2015a). Gene therapy for deafness: How close are we? Sci. Transl. Med. 7, 295fs28$295 \mathrm{fs} 28$.

Moser, T. (2015b). Optogenetic stimulation of the auditory pathway for research and future prosthetics. Curr. Opin. Neurobiol. 34, 29-36.

Nagel, G., Ollig, D., Fuhrmann, M., Kateriya, S., Musti, A.M., Bamberg, E., and Hegemann, P. (2002). Channelrhodopsin-1: a light-gated proton channel in green algae. Science 296, 2395-2398.

Nagel, G., Szellas, T., Huhn, W., Kateriya, S., Adeishvili, N., Berthold, P., Ollig, D., Hegemann, P., and Bamberg, E. (2003). Channelrhodopsin-2, a directly light-gated cation-selective membrane channel. Proc. Natl. Acad. Sci. 100, 13940-13945.

Oshima, K., Shin, K., Diensthuber, M., Peng, A.W., Ricci, A.J., and Heller, S. (2010). Mechanosensitive hair cell-like cells from embryonic and induced pluripotent stem cells. Cell 141, 704-716.

Pan, B., Askew, C., Galvin, A., Heman-Ackah, S., Asai, Y., Indzhykulian, A.A., Jodelka, F.M., Hastings, M.L., Lentz, J.J., Vandenberghe, L.H., et al. (2017). Gene therapy restores auditory and vestibular function in a mouse model of Usher syndrome type 1c. Nat. Biotechnol. 35, 264-272.

Pangrsic, T., Lasarow, L., Reuter, K., Takago, H., Schwander, M., Riedel, D., Frank, T., Tarantino, L.M., Bailey, J.S., Strenzke, N., et al. (2010). Hearing requires otoferlin-dependent efficient replenishment of synaptic vesicles in hair cells. Nat. Neurosci. 13, 869-876.

Pinyon, J.L., Tadros, S.F., Froud, K.E., Y Wong, A.C., Tompson, I.T., Crawford, E.N., Ko, M., Morris, R., Klugmann, M., and Housley, G.D. (2014). Close-field electroporation gene delivery using the cochlear implant electrode array enhances the bionic ear. Sci. Transl. Med. 6, 233ra54-233ra54. 
Remington, E.D., Osmanski, M.S., and Wang, X. (2012). An operant conditioning method for studying auditory behaviors in marmoset monkeys. PloS One 7, e47895.

Richter, C.-P., Rajguru, S.M., Matic, A.I., Moreno, E.L., Fishman, A.J., Robinson, A.M., Suh, E., and Walsh, J.T. (2011). Spread of cochlear excitation during stimulation with pulsed infrared radiation: inferior colliculus measurements. J. Neural Eng. 8, 056006.

Robertson, D., and Irvine, D.R. (1989). Plasticity of frequency organization in auditory cortex of guinea pigs with partial unilateral deafness. J. Comp. Neurol. 282, 456-471.

Rubinstein, J.T. (2004). How cochlear implants encode speech. Curr. Opin. Otolaryngol. Head Neck Surg. 12, 444-448.

Russell, I., and Sellick, P. (1983). Low-frequency characteristics of intracellularly recorded receptor potentials in guinea-pig cochlear hair cells. J. Physiol. 338, 179-206.

Schmiedt, R.A. (1989). Spontaneous rates, thresholds and tuning of auditory-nerve fibers in the gerbil: comparisons to cat data. Hear. Res. 42, 23-35.

Schneider-Warme, F. (2018). The power of optogenetics : Potential in cardiac experimental and clinical electrophysiology. Herzschrittmachertherapie Elektrophysiologie 29, 24-29.

Scholl, H.P.N., Strauss, R.W., Singh, M.S., Dalkara, D., Roska, B., Picaud, S., and Sahel, J.-A. (2016). Emerging therapies for inherited retinal degeneration. Sci. Transl. Med. 8, 368rv6.

Schultz, M., Baumhoff, P., Maier, H., Teudt, I.U., Krüger, A., Lenarz, T., and Kral, A. (2012). Nanosecond laser pulse stimulation of the inner ear-a wavelength study. Biomed. Opt. Express 3, 3332-3345.

Schwaerzle, M., Nehlich, J., Ayub, S., Paul, O., and Ruther, P. (2016). Led-based optical cochlear implant on highly flexible triple layer polyimide substrates. In 2016 IEEE 29th International Conference on Micro Electro Mechanical Systems (MEMS), (Shanghai, China: IEEE), pp. 395-398.

Sengupta, A., Chaffiol, A., Macé, E., Caplette, R., Desrosiers, M., Lampič, M., Forster, V., Marre, O., Lin, J.Y., Sahel, J.-A., et al. (2016). Red-shifted channelrhodopsin stimulation restores light responses in blind mice, macaque retina, and human retina. EMBO Mol. Med. 8, 1248-1264.

Senova, S., Scisniak, I., Chiang, C.-C., Doignon, I., Palfi, S., Chaillet, A., Martin, C., and Pain, F. (2017). Experimental assessment of the safety and potential efficacy of high irradiance photostimulation of brain tissues. Sci. Rep. 7, 43997.

Sewell, W.F. (1984). The effects of furosemide on the endocochlear potential and auditory-nerve fiber tuning curves in cats. Hear. Res. 14, 305-314.

Shannon, R.V. (1983). Multichannel electrical stimulation of the auditory nerve in man. II. Channel interaction. Hear. Res. 12, 1-16.

Shannon, R.V., Zeng, F.G., Kamath, V., Wygonski, J., and Ekelid, M. (1995). Speech recognition with primarily temporal cues. Science 270, 303-304. 
Shannon, R.V., Cruz, R.J., and Galvin, J.J. (2011). Effect of Stimulation Rate on Cochlear Implant Users' Phoneme, Word and Sentence Recognition in Quiet and in Noise. Audiol. Neurotol. 16, 113-123.

Shen, Y., Ye, B., Chen, P., Wang, Q., Fan, C., Shu, Y., and Xiang, M. (2018). Cognitive Decline, Dementia, Alzheimer's Disease and Presbycusis: Examination of the Possible Molecular Mechanism. Front. Neurosci. 12, 394.

Shevchenko, V., Mager, T., Kovalev, K., Polovinkin, V., Alekseev, A., Juettner, J., Chizhov, I., Bamann, C., Vavourakis, C., Ghai, R., et al. (2017). Inward H+ pump xenorhodopsin: Mechanism and alternative optogenetic approach. Sci. Adv. 3.

Shimano, T., Fyk-Kolodziej, B., Mirza, N., Asako, M., Tomoda, K., Bledsoe, S., Pan, Z.H., Molitor, S., and Holt, A.G. (2013). Assessment of the AAV-mediated expression of channelrhodopsin-2 and halorhodopsin in brainstem neurons mediating auditory signaling. Brain Res. 1511, 138-152.

Shin, G., Gomez, A.M., Al-Hasani, R., Jeong, Y.R., Kim, J., Xie, Z., Banks, A., Lee, S.M., Han, S.Y., Yoo, C.J., et al. (2017). Flexible Near-Field Wireless Optoelectronics as Subdermal Implants for Broad Applications in Optogenetics. Neuron 93, 509-521.e3.

Shnerson, A., Devigne, C., and Pujol, R. (1981). Age-related changes in the C57BL/6J mouse cochlea. II. Ultrastructural findings. Brain Res. 254, 77-88.

Shrestha, B.R., Chia, C., Wu, L., Kujawa, S.G., Liberman, M.C., and Goodrich, L.V. (2018). Sensory Neuron Diversity in the Inner Ear Is Shaped by Activity. Cell 174, 1229-1246.e17.

Simonelli, F., Maguire, A.M., Testa, F., Pierce, E.A., Mingozzi, F., Bennicelli, J.L., Rossi, S., Marshall, K., Banfi, S., Surace, E.M., et al. (2010). Gene therapy for Leber's congenital amaurosis is safe and effective through 1.5 years after vector administration. Mol. Ther. J. Am. Soc. Gene Ther. 18, 643650.

Snyder, R.L., Bierer, J.A., and Middlebrooks, J.C. (2004). Topographic spread of inferior colliculus activation in response to acoustic and intracochlear electric stimulation. J. Assoc. Res. Otolaryngol. JARO 5, 305-322.

Steel, K.P. (2000). Science, medicine, and the future: New interventions in hearing impairment. BMJ 320, 622-625.

Stockklausner, C., Ludwig, J., Ruppersberg, J.P., and Klöcker, N. (2001). A sequence motif responsible for ER export and surface expression of Kir2.0 inward rectifier $\mathrm{K}(+)$ channels. FEBS Lett. 493, 129-133.

Sun, S., Babola, T., Pregernig, G., So, K.S., Nguyen, M., Su, S.-S.M., Palermo, A.T., Bergles, D.E., Burns, J.C., and Müller, U. (2018). Hair Cell Mechanotransduction Regulates Spontaneous Activity and Spiral Ganglion Subtype Specification in the Auditory System. Cell 174, 1247-1263.e15.

Suzuki, J., Hashimoto, K., Xiao, R., Vandenberghe, L.H., and Liberman, M.C. (2017). Cochlear gene therapy with ancestral AAV in adult mice: complete transduction of inner hair cells without cochlear dysfunction. Sci. Rep. 7, 45524.

Tao, C., Zhang, G., Zhou, C., Wang, L., Yan, S., Zhou, Y., and Xiong, Y. (2017). Bidirectional Shifting Effects of the Sound Intensity on the Best Frequency in the Rat Auditory Cortex. Sci. Rep. 7. 
Terreros, G., and Delano, P.H. (2015). Corticofugal modulation of peripheral auditory responses. Front. Syst. Neurosci. 9.

Thompson, A.C., Fallon, J.B., Wise, A.K., Wade, S.A., Shepherd, R.K., and Stoddart, P.R. (2015). Infrared neural stimulation fails to evoke neural activity in the deaf guinea pig cochlea. Hear. Res. $324,46-53$.

Tomlinson, R.W., and Schwarz, D.W. (1988). Perception of the missing fundamental in nonhuman primates. J Acoust Soc Am 84, 560-565.

Tønnesen, J., and Kokaia, M. (2017). Epilepsy and optogenetics: can seizures be controlled by light? Clin. Sci. Lond. Engl. 1979 131, 1605-1616.

Uchida, Y., Sugiura, S., Nishita, Y., Saji, N., Sone, M., and Ueda, H. (2019). Age-related hearing loss and cognitive decline - The potential mechanisms linking the two. Auris. Nasus. Larynx 46, 1-9.

Verma, R.U., Guex, A.A., Hancock, K.E., Durakovic, N., McKay, C.M., Slama, M.C.C., Brown, M.C., and Lee, D.J. (2014). Auditory responses to electric and infrared neural stimulation of the rat cochlear nucleus. Hear. Res. 310, 69-75.

Viemeister, N.F. (1988). Intensity coding and the dynamic range problem. Hear. Res. 34, 267-274.

Wang, X., and Walker, K.M.M. (2012). Neural mechanisms for the abstraction and use of pitch information in auditory cortex. J. Neurosci. Off. J. Soc. Neurosci. 32, 13339-13342.

White, P.M., Doetzlhofer, A., Lee, Y.S., Groves, A.K., and Segil, N. (2006). Mammalian cochlear supporting cells can divide and trans-differentiate into hair cells. Nature 441, 984-987.

Wietek, J., Wiegert, J.S., Adeishvili, N., Schneider, F., Watanabe, H., Tsunoda, S.P., Vogt, A., Elstner, M., Oertner, T.G., and Hegemann, P. (2014). Conversion of channelrhodopsin into a lightgated chloride channel. Science 344, 409-412.

Willett, K., and Bennett, J. (2013). Immunology of AAV-Mediated Gene Transfer in the Eye. Front. Immunol. 4, 261.

Wilson, B.S., Finley, C.C., Lawson, D.T., Wolford, R.D., Eddington, D.K., and Rabinowitz, W.M. (1991). Better speech recognition with cochlear implants. Nature 352, 236-238.

Wrobel, C., Dieter, A., Huet, A., Keppeler, D., Duque-Afonso, C.J., Vogl, C., Hoch, G., Jeschke, M., and Moser, T. (2018). Optogenetic stimulation of cochlear neurons activates the auditory pathway and restores auditory-driven behavior in deaf adult gerbils. Sci. Transl. Med. 10, eaao0540.

Xu, Y., Xia, N., Lim, M., Tan, X., Tran, M.H., Boulger, E., Peng, F., Young, H., Rau, C., Rack, A., et al. (2018). Multichannel optrodes for photonic stimulation. Neurophotonics 5, 045002.

Yizhar, O., Fenno, L.E., Davidson, T.J., Mogri, M., and Deisseroth, K. (2011). Optogenetics in neural systems. Neuron 71, 9-34.

Young, H.K., Tan, X., Xia, N., and Richter, C.-P. (2015). Target structures for cochlear infrared neural stimulation. Neurophotonics 2, 025002.

Zeng, F.-G. (2004). Trends in cochlear implants. Trends Amplif. 8, 1-34. 
Zeng, F.-G., and Galvin, J.J.I. (1999). Amplitude Mapping and Phoneme Recognition in Cochlear Implant Listeners. Ear Hear. 20.

Zeng, F.-G., Rebscher, S., Harrison, W.V., Sun, X., and Feng, H. (2008). Cochlear Implants:System Design, Integration and Evaluation. IEEE Rev. Biomed. Eng. 1, 115-142.

Zhang, Y.-P., and Oertner, T.G. (2007). Optical induction of synaptic plasticity using a lightsensitive channel. Nat. Methods 4, 139-141.

Zhang, J., Tian, J., Ta, N., and Rao, Z. (2018a). Transient response of the human ear to impulsive stimuli: A finite element analysis. J. Acoust. Soc. Am. 143, 2768-2779.

Zhang, W., Kim, S.M., Wang, W., Cai, C., Feng, Y., Kong, W., and Lin, X. (2018b). Cochlear Gene Therapy for Sensorineural Hearing Loss: Current Status and Major Remaining Hurdles for Translational Success. Front. Mol. Neurosci. 11.

Zierhofer, C.M., Hochmair-Desoyer, I.J., and Hochmair, E.S. (1995). Electronic design of a cochlear implant for multichannel high-rate pulsatile stimulation strategies. IEEE Trans. Rehabil. Eng. 3, 112-116.

Zinchenko, A., Kanske, P., Obermeier, C., Schröger, E., Villringer, A., and Kotz, S.A. (2018). Modulation of Cognitive and Emotional Control in Age-Related Mild-to-Moderate Hearing Loss. Front. Neurol. 9, 783.

Zinn, E., Pacouret, S., Khaychuk, V., Turunen, H.T., Carvalho, L.S., Andres-Mateos, E., Shah, S., Shelke, R., Maurer, A.C., Plovie, E., et al. (2015). In Silico Reconstruction of the Viral Evolutionary Lineage Yields a Potent Gene Therapy Vector. Cell Rep. 12, 1056-1068.

Zorzos, A.N., Boyden, E.S., and Fonstad, C.G. (2010). Multiwaveguide implantable probe for light delivery to sets of distributed brain targets. Opt. Lett. 35, 4133. 


\section{Acknowledgements}

Zuallererst möchte ich mich bei dir, Tobias, für die Ermöglichung meiner Doktorarbeit bedanken. Danke für das Mentoring und meine Förderung, sowie für das mir entgegengebrachte Vertrauen und eingeräumte Freiheiten. Durch dein präzises und kritisches (aber stets konstruktives) Feedback sowie Antworten in Minutenschnelle hat mir die Zusammenarbeit mit dir sehr viel Spaß gemacht. Ich bin sowohl inhaltlich als auch im Ergebnis sehr zufrieden mit meiner Doktorarbeit, was ich zum allergrößten Teil dir zu verdanken habe. Ich Danke dir vielmals für die Jahre der Zusammenarbeit und würde mich sehr freuen, wenn wir auch in Zukunft noch miteinander in Kontakt stehen werden.

Marcus, ich möchte dir besonders für die endlosen Diskussionen, deine Hilfestellungen in der praktischen Arbeit, sowie stets konstruktive Kritik bezüglich wissenschaftlichen Arbeitens danken. Du bist mit deiner Art zu Denken und deiner Arbeitsweise in mehrerlei Hinsicht ein inspirierendes und motivierendes (lies: stabiles) Vorbild für mich geworden und ich hoffe, dass wir noch eine ganze Weile über den Rahmen meiner Doktorarbeit hinaus miteinander zu tun haben werden.

Captain, ich danke dir für meine Einarbeitung und Integration am Institut vom ersten Tag meiner Laborrotation an, sowie für stetige Diskussionen und Feedback. Du warst nicht nur ein super Kollege, sondern bist in den letzten Jahren zu einem wichtigen Freund für mich geworden, auf den man sich verlassen und mit dem man sich aus dem ein oder anderen Tiefpunkt gemeinsam hochziehen kann.

Christian, ich danke dir vielmals für das geduldige Einarbeiten zu Beginn meiner Doktorarbeit. Dein fliegender Umgang mit dem Skalpell, dein Optimismus sowie dein „jetzt mach das einfach“ haben mich sehr motiviert. Ich danke dir für die vielen Stunden am Setup, im Fiz, und am Tresen. Furthermore, I would like to thank Antoine, Thomas, and Dom for countless critical discussions on experimental design, methodological improvements and data interpretation, and Fritz for regularly remining me not to forget about the existence of a different perspective.

Ich danke euch, Gerhard, Lukasz, Tamas, Peter und Daniel, für eure geduldigen Erklärungen und Hilfestellung bei technischen Fragen aller Art sowie das Implementieren von Lösungen für alle erdenklichen Probleme die mir die letzten Jahre in die Quere kamen. 
Ich danke euch, Christane, Sandra, Daniela und Nadine, für stetige Unterstützung der experimentellen sowie administrativen Arbeit - ihr habt mein Leben im Labor um einiges erleichtert. Außerdem danke ich dir, Patricia, für deine ergebnisorientierte Hilfe bei Problemen aller Art sowie das Ermöglichen von Terminen auf den letzten Drücker. Auch wenn es schwer fällt sich das vorzustellen, werde ich mir in Zukunft bestimmt das ein oder andere R wünschen.

Ich danke Carsten Lott, Joachim Niechoy und Rainer Schürkötter von den wissenschaftlichen Werkstätten der UMG und des MPI-BPC für die hervorrgande technische Beratung und Hilfe während der letzten Jahre.

Außerdem danke ich Eric Klein und Patrick Ruther vom Institut für Mikrosystemtechnik in Freiburg sowie Roland Hessler von MED-EL für Design, Fertigung und Bereitstellung der optischen und elektrischen Cochela-Implantate, welche in dieser Doktorarbeit zur Anwendung kamen.

Finally, I would like to express my deepest gratitude to all co-authors of scientific publications that I was able to contribute to and that still might come in the future. It was a big pleasure to work with and learn from you, and without you my PhD would not have been as fruitful as I consider it to be.

Abgesehen von der wissenschaftlichen Seite gilt mein ganz besonderer Dank Andreas Becker, der in der Zentralmensa nicht nur die Rindersteaks immer exakt auf den Punkt gegrillt hat, sondern auch außergewöhnlichere Köstlichkeiten wie Flugentenbrust und Lammhüfte aus dem Hut zauberte, und mich so des Öfteren wohlgenährt und zufrieden in die zweite Hälfte des Tages starten lies.

Ich danke euch, Mama und Papa, für eure stetige Unterstützung, nicht nur während meiner Doktorarbeit. Ohne euren Zuspruch hätte ich nie angefangen zu studieren, und ohne euren Rückhalt hätte ich den Kopf nicht so frei gehabt, wie ich ihn hatte - und ich wäre mit Sicherheit nicht so glücklich mit meiner aktuellen Situation wie ich es jetzt bin. Danke für Alles!

Julia, du hast mich nun beinahe durch die gesamte Doktorarbeit begleitet und viele Hoch- und Tiefpunkte miterlebt. Danke, dass du mir immer wieder einen passenden Platz zwischen Antriebslosigkeit und Übermut gezeigt hast, mich aufgebaut und geerdet hast. Ich bin dir dankbar für all die Momente die wir miteinander hatten, und freue mich auf jeden, der noch kommen wird. 


\section{Abbreviations}

Al

aABR

AAV

ABR

ANF

ANOVA

BE

BF

CatCh

CF

ChR

ChR2

$\mathrm{Cl}$

$d^{\prime}$

$\mathrm{dB}$

dB SPL

DR

eABR

$\mathrm{eCl}$

eGFP

EDTA

ES

eYFP

FDA

GaN

GC

HEK

HI

IC

ICC

hSyn

Kir primary auditory cortex

acoustically evoked auditory brainstem response

adeno-associated virus

auditory brainstem response

auditory nerve fiber

analysis of variance

best electrode

best frequency

calcium translocating channelrhodopsin

characteristic frequency

channelrhodopsin

channelrhodopsin-2

cochlear implant

d-prime, sensitivity-index

decibel

decibel sound pressure level

dynamic range

electrically evoked auditory brainstem response

electrical cochlear implant

enhanced green fluorescent protein

ethylenediaminetetraacetic acid

export signal

enhanced yellow fluorescent protein

food and drug administration

gallium nitride

genome copies

human embryonic kidney (cell)

hearing impairment

inferior colliculus

central nucleus of the inferior colliculus

human synapsin

inward-rectifying potassium channel 
LED

MUA

$\mu L E D$

NA

PBS

PFA

PSTH

SD

SEM

SGN

SNHL

SPL

STC

Thy1

TS

OABR

$\mathrm{oCl}$

RW

VS light-emitting diode

multi-unit activity

microscale light-emitting diode

numerical aperture

phosphate buffered saline

paraformaldehyde

peri-stimulus time histogram

standard deviation

standard error of the mean

spiral ganglion neuron

sensorineural hearing loss

sound pressure level

spatial tuning curve

thymocyte differentiation antigen 1

trafficking signal

optically evoked auditory brainstem response

optogenetic cochlear implant

round window

vector strength 


\section{Declaration}

Herewith I declare that my dissertation, entitled "Towards Optical Cochlear Implants: Behavioral and Physiological Responses to Optogenetic Activation of the Auditory Nerve", has been written on my own and with no other sources and aids than quoted. 